\title{
Homoleptic Imidophosphorane Stabilization of Tetravalent Cerium
}

\section{Supporting Information}

Natalie T. Rice, ${ }^{\dagger} \xi$ Jing Su, ${ }^{\phi} \xi$ Thaige P. Gompa, ${ }^{\dagger}$ Dominic R. Russo, ${ }^{\dagger}$ Joshua Telser,${ }^{\S}$ Lukas Palatinus, ${ }^{\#}$ John Bacsa, ${ }^{\dagger}$ Ping Yang, ${ }^{*} \phi$ Enrique R. Batista, ${ }^{* \phi}$ and Henry S. La Pierre*t\#

†School of Chemistry and Biochemistry and $\neq$ Nuclear and Radiological Engineering Program, Georgia Institute of Technology, Atlanta, Georgia 30332-0400, United States ${ }^{\phi}$ Theoretical Division, Los Alamos National Laboratory, Los Alamos, New Mexico 87545, United States

\$Department of Biological, Chemical and Physical Sciences, Roosevelt University, Chicago, Illinois 60605, United States

\#Institute of Physics, Czech Academy of Sciences, Na Slovance 2, 182 21, Prague 6, Czechia

§N.T.R. and J.S. contributed equally to this work.

Table of Contents

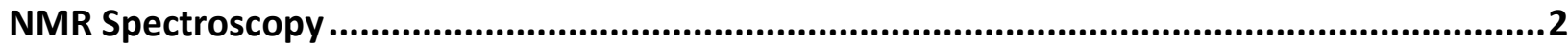

Crystallographic Analyses.....................................................................................................

Electronic Absorption Spectra ..................................................................................................35

Magnetism .........................................................................................................................

Theoretical Calculations ..........................................................................................................38

Chemical Reactivity .............................................................................................................

Cyclic Voltammetry ..................................................................................................................5

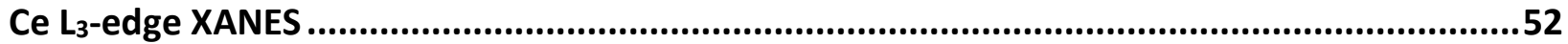


NMR Spectroscopy

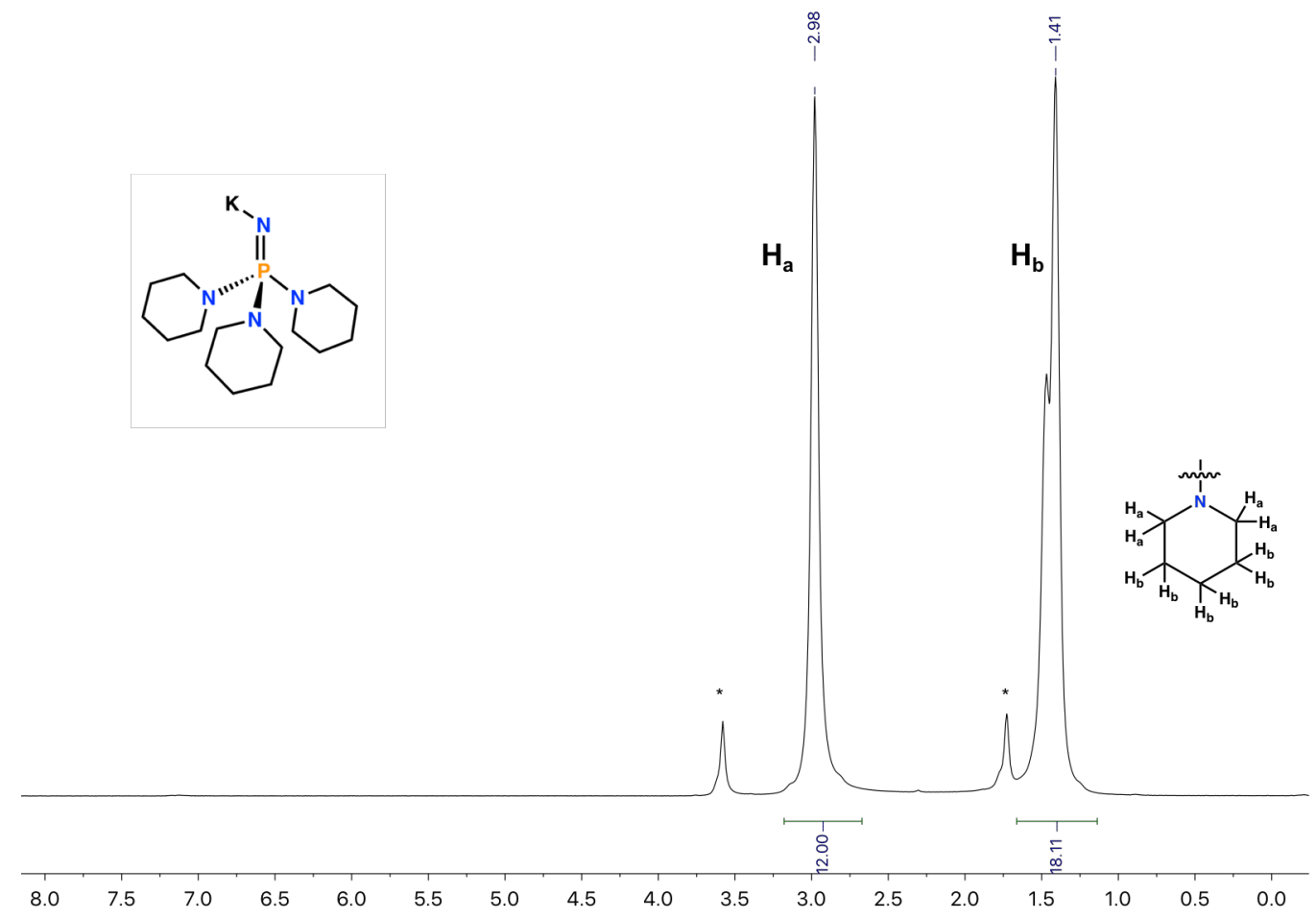

Figure S1. ${ }^{1} \mathrm{H}$ NMR of $1-\mathrm{K}$ in $d_{8}-\mathrm{THF}$. Peaks of $\mathrm{OC}_{4} \mathrm{D}_{7} \mathrm{H}$ noted as *.

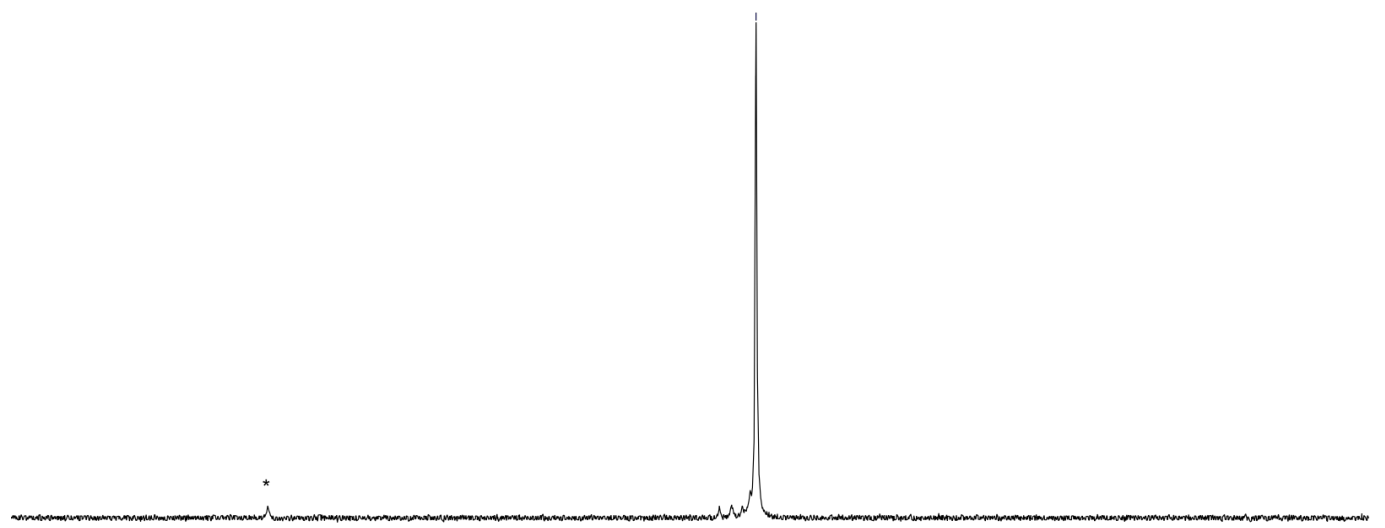

$\begin{array}{lllllllllllllllllll}50 & 45 & 40 & 35 & 30 & 25 & 20 & 15 & 10 & 5 & 0 & -5 & -10 & -15 & -20 & -25 & -30 & -35 & -40\end{array}$ Figure S2. ${ }^{31} \mathrm{P}$ NMR of $\mathbf{1 - K}$ in $d_{8}$-THF. Residual imine denoted by *. 


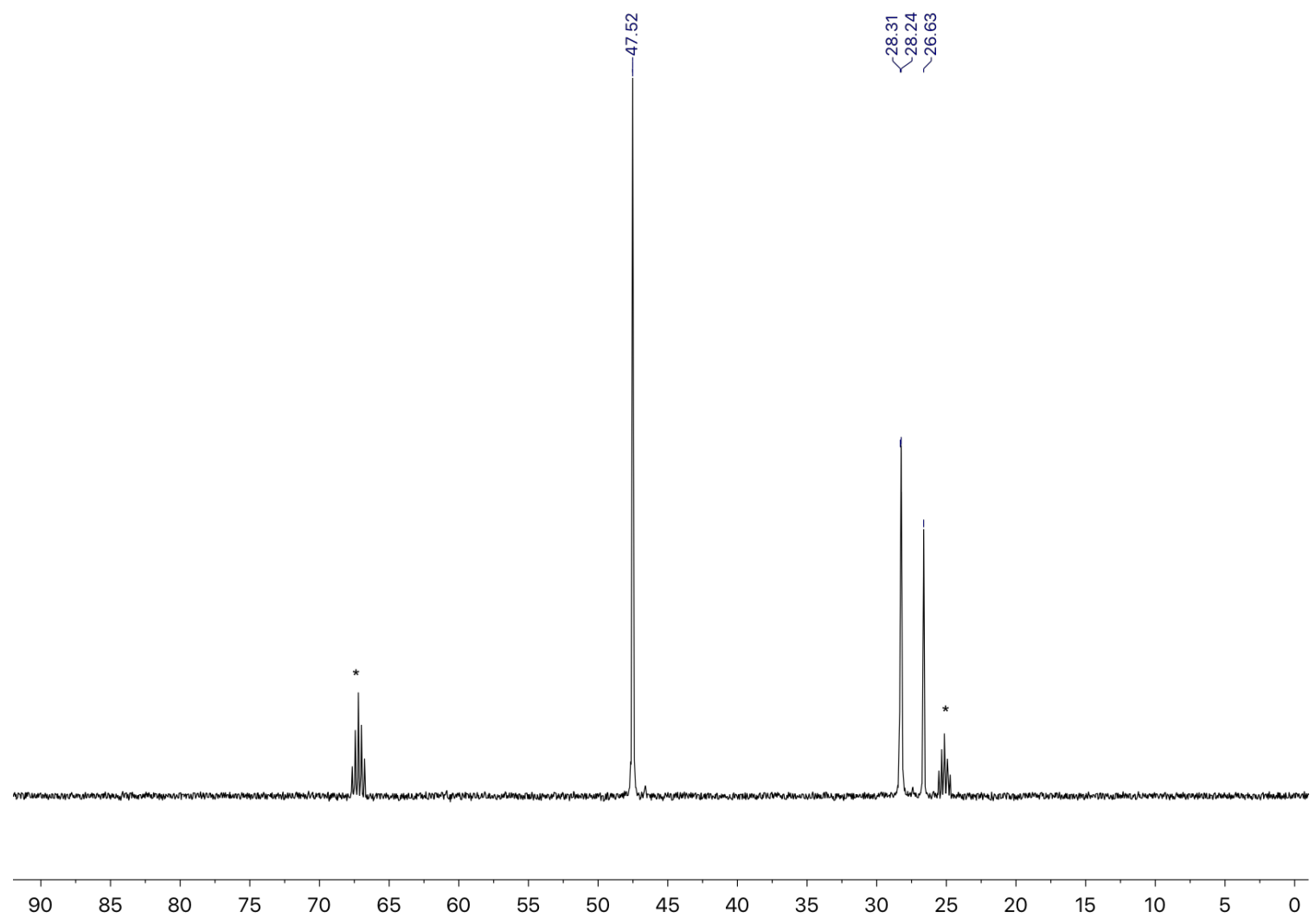

Figure S3. ${ }^{13} \mathrm{C}$ NMR of $1-\mathrm{K}$ in $d_{8}-\mathrm{THF}$. Peak of $\mathrm{OC}_{4} \mathrm{D}_{7} \mathrm{H}$ is noted as *.
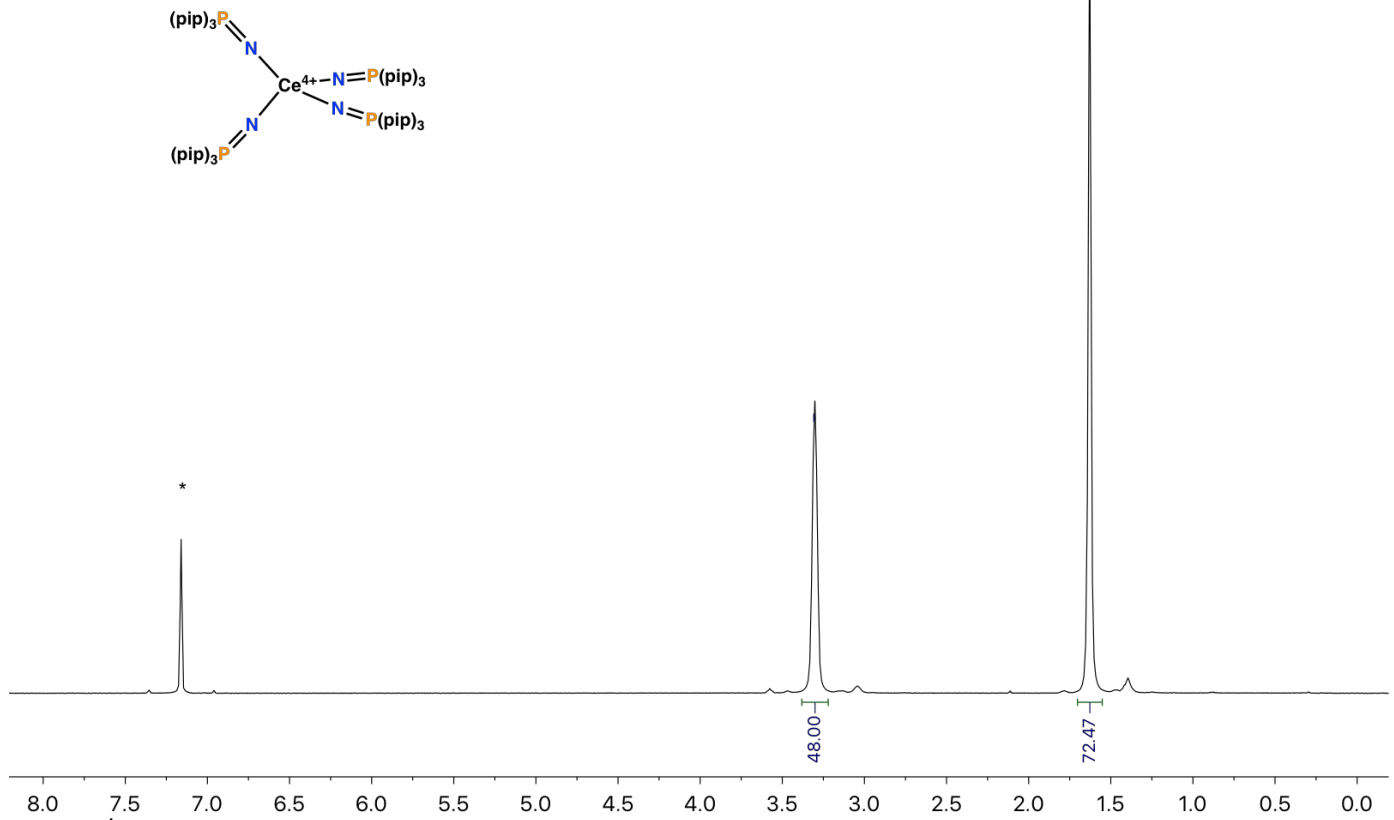

Figure S4. ${ }^{1} \mathrm{H}$ NMR of $2-\mathrm{Ce}$ in $\mathrm{C}_{6} \mathrm{D}_{6}$. Peak of $\mathrm{C}_{6} \mathrm{D}_{5} \mathrm{H}$ is noted as *. 


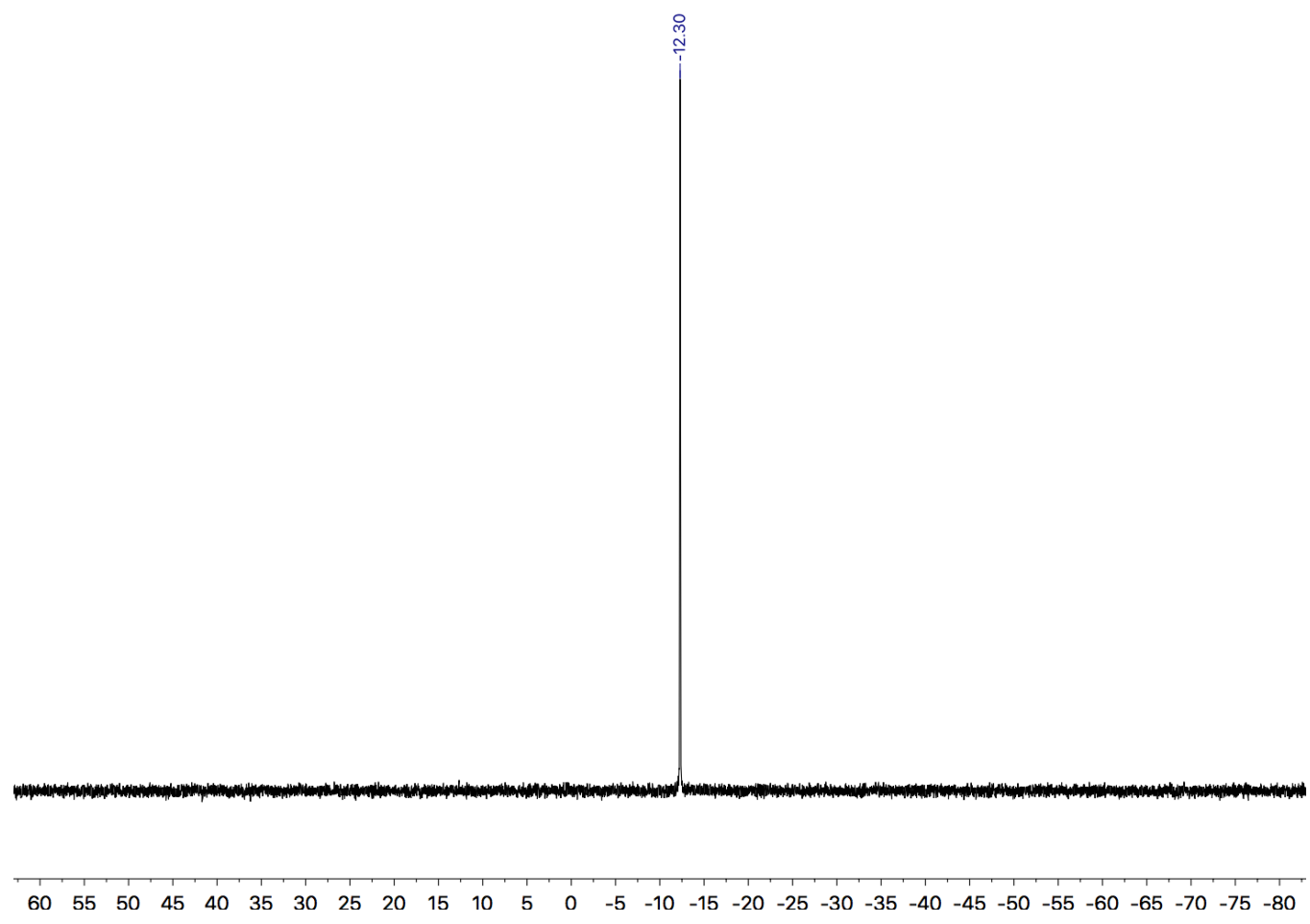

Figure S5. ${ }^{31} \mathrm{P}$ NMR of $2-\mathrm{Ce}$ in $\mathrm{C}_{6} \mathrm{D}_{6}$.

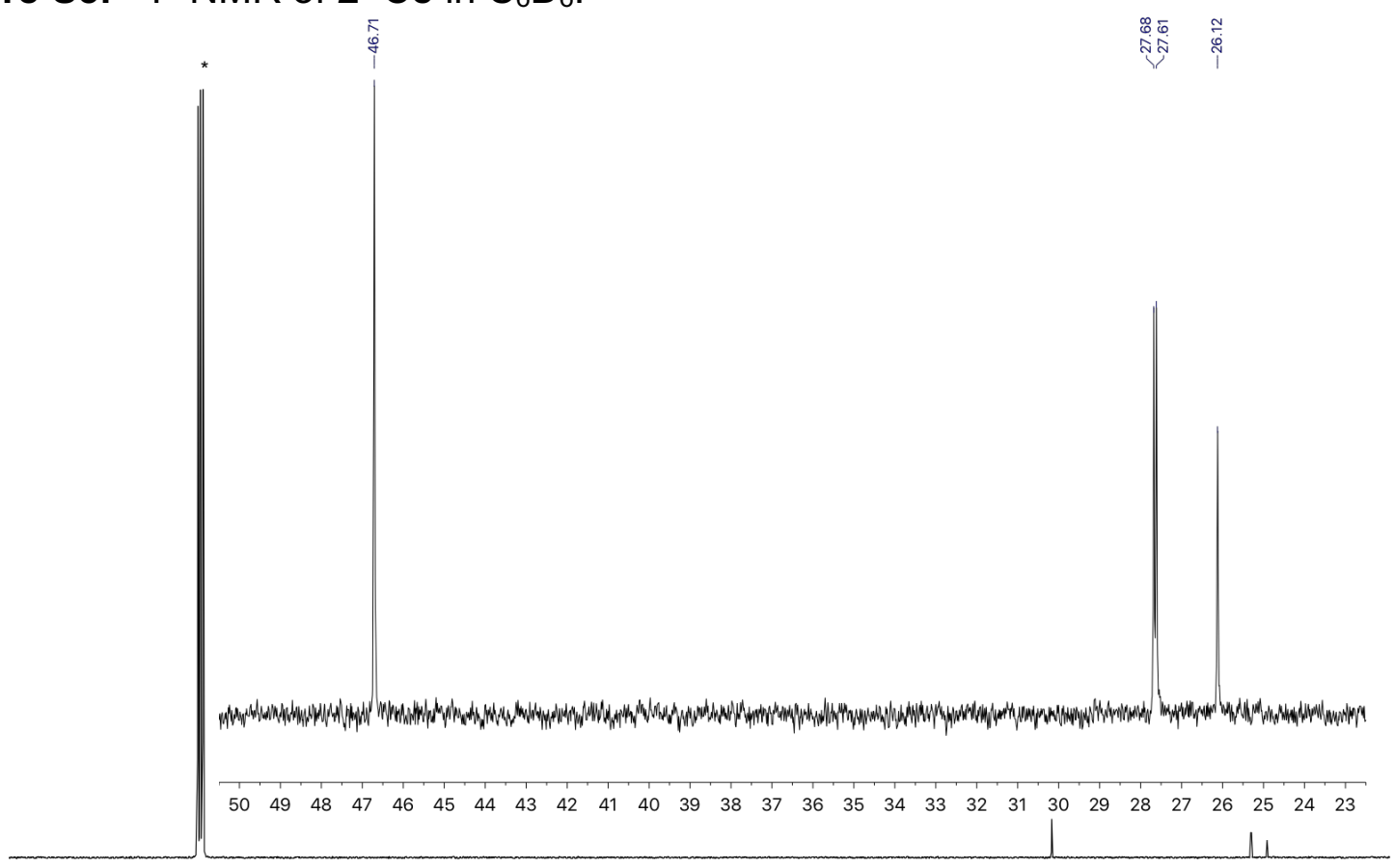

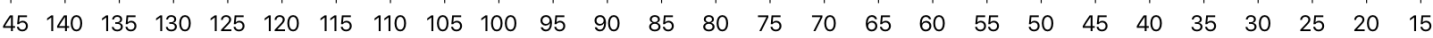

Figure S6. ${ }^{13} \mathrm{C}$ NMR of $2-\mathrm{Ce}$ in $\mathrm{C}_{6} \mathrm{D}_{6}$. Peak of $\mathrm{C}_{6} \mathrm{D}_{5} \mathrm{H}$ is noted as *. 


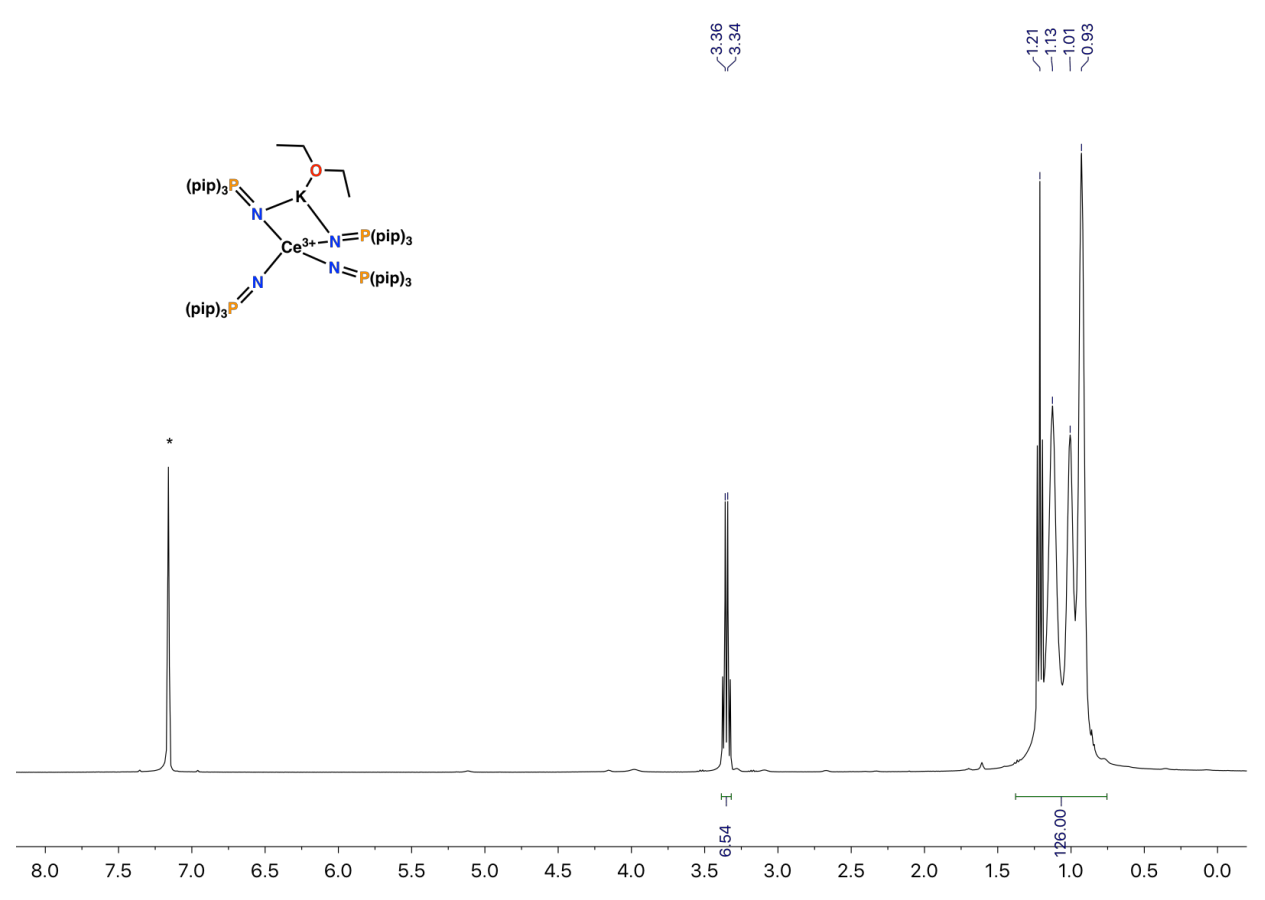

Figure S7A. ${ }^{1} \mathrm{H}$ NMR of $3-\mathrm{Ce}-\mathrm{K}\left(\mathrm{Et}_{2} \mathrm{O}\right)$ in $\mathrm{C}_{6} \mathrm{D}_{6}$. Peak of $\mathrm{C}_{6} \mathrm{D}_{5} \mathrm{H}$ is noted as *. Between one and two diethyl ether molecules is shown bound to the complex after exposure of the isolated material to dynamic vacuum.
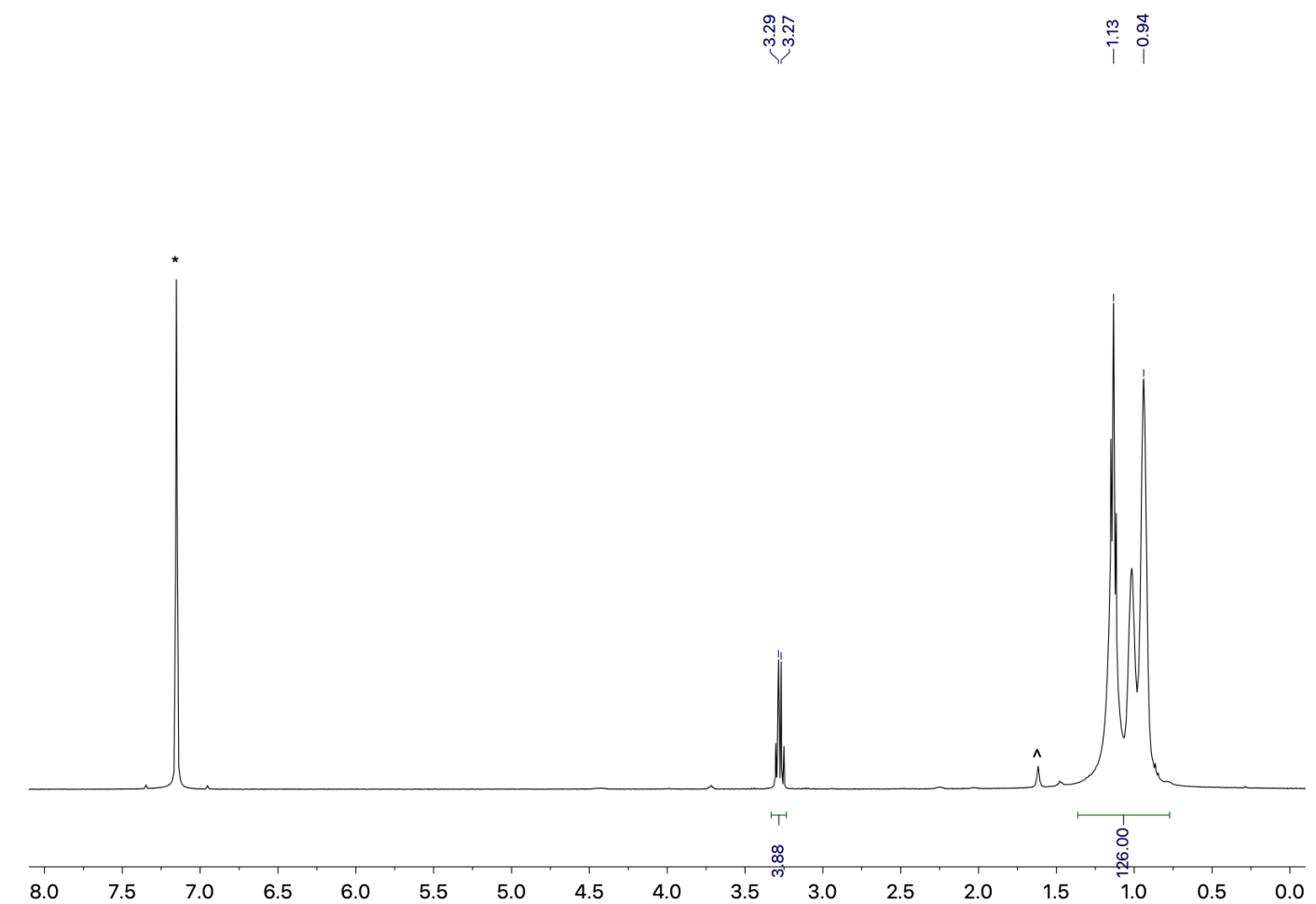

Figure S7B. ${ }^{1} \mathrm{H}$ NMR of $3-\mathrm{Ce}-\mathbf{K}\left(\mathrm{Et}_{2} \mathrm{O}\right)$ in $\mathrm{C}_{6} \mathrm{D}_{6}$. Peak of $\mathrm{C}_{6} \mathrm{D}_{5} \mathrm{H}$ is noted as ${ }^{*}$. Between one and two diethyl ether molecules is shown bound to the complex after exposure of the isolated material to dynamic vacuum. 


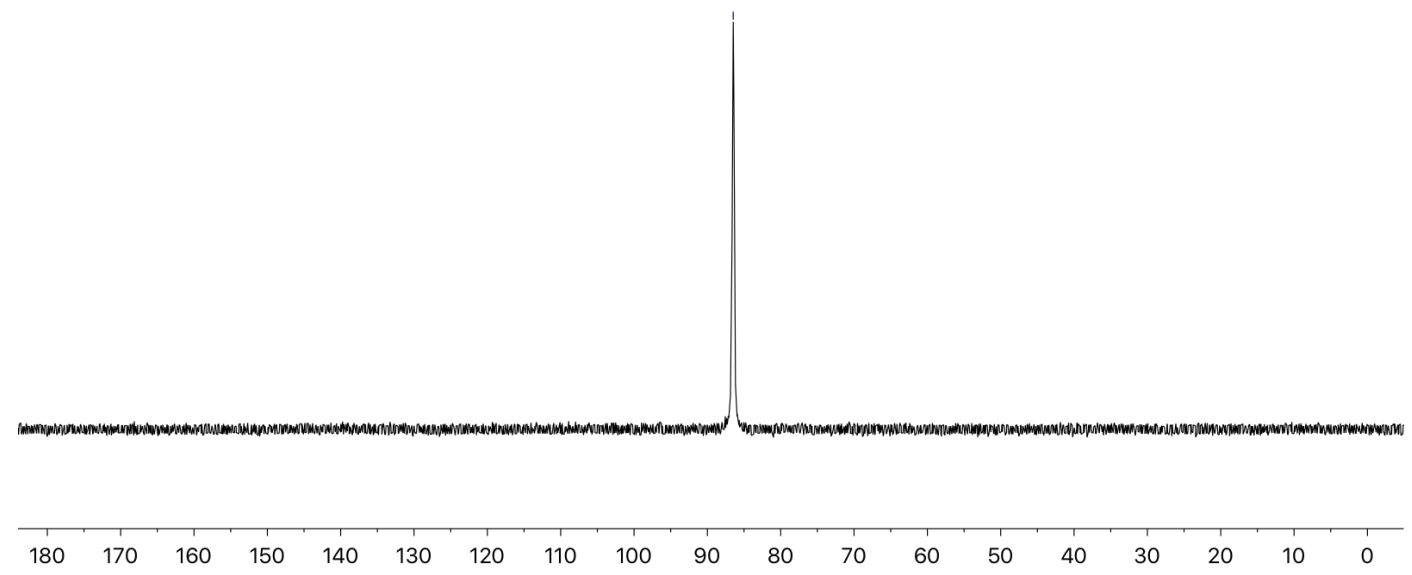

Figure S8. ${ }^{31} \mathrm{P}$ NMR of $3-\mathrm{Ce}-\mathrm{K}\left(\mathrm{Et}_{2} \mathrm{O}\right)$ in $\mathrm{C}_{6} \mathrm{D}_{6}$.
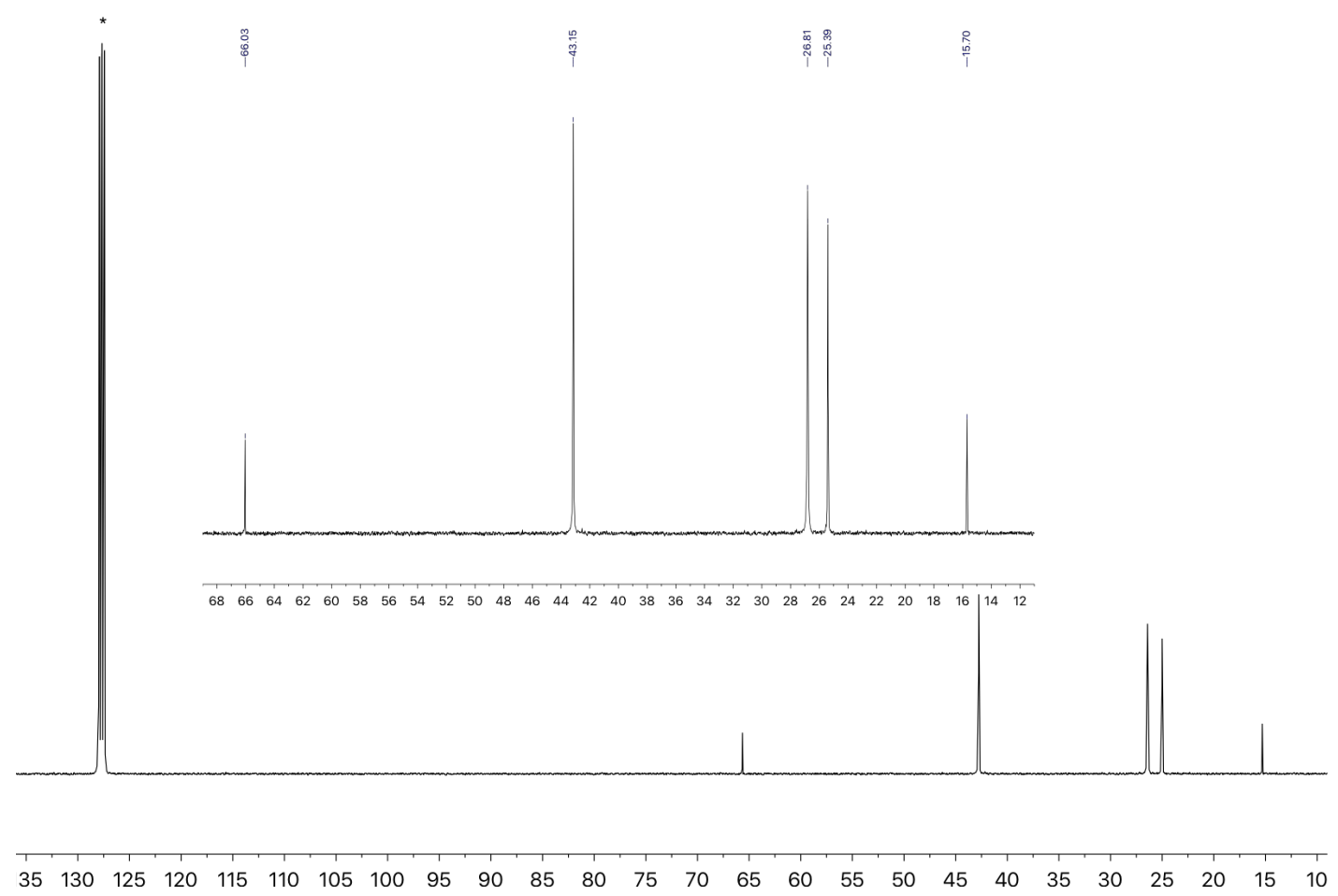

Figure S9. ${ }^{13} \mathrm{C}$ NMR of $3-\mathrm{Ce}-\mathrm{K}\left(\mathrm{Et}_{2} \mathrm{O}\right)$ in $\mathrm{C}_{6} \mathrm{D}_{6}$. Peak of $\mathrm{C}_{6} \mathrm{D}_{5} \mathrm{H}$ is noted as *. 
$25 \circ C$

$\mathbf{0} \circ \mathrm{C}$

$-15 \circ C$

$-30 \cdot C$

$-45 \circ C$

$-60 \cdot \mathrm{C}$

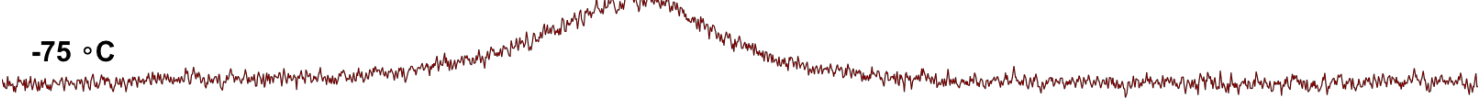

$\begin{array}{lllllllllllllllllllll}185 & 180 & 175 & 170 & 165 & 160 & 155 & 150 & 145 & 140 & 135 & 130 & 125 & 120 & 115 & 110 & 105 & 100 & 95 & 90 & 85\end{array}$ Figure S10. Variable temperature ${ }^{31} \mathrm{P}$ NMR of $3-\mathrm{Ce}-\mathrm{K}\left(\mathrm{Et}_{2} \mathrm{O}\right)$ in $\mathrm{C}_{6} \mathrm{D}_{6}$.
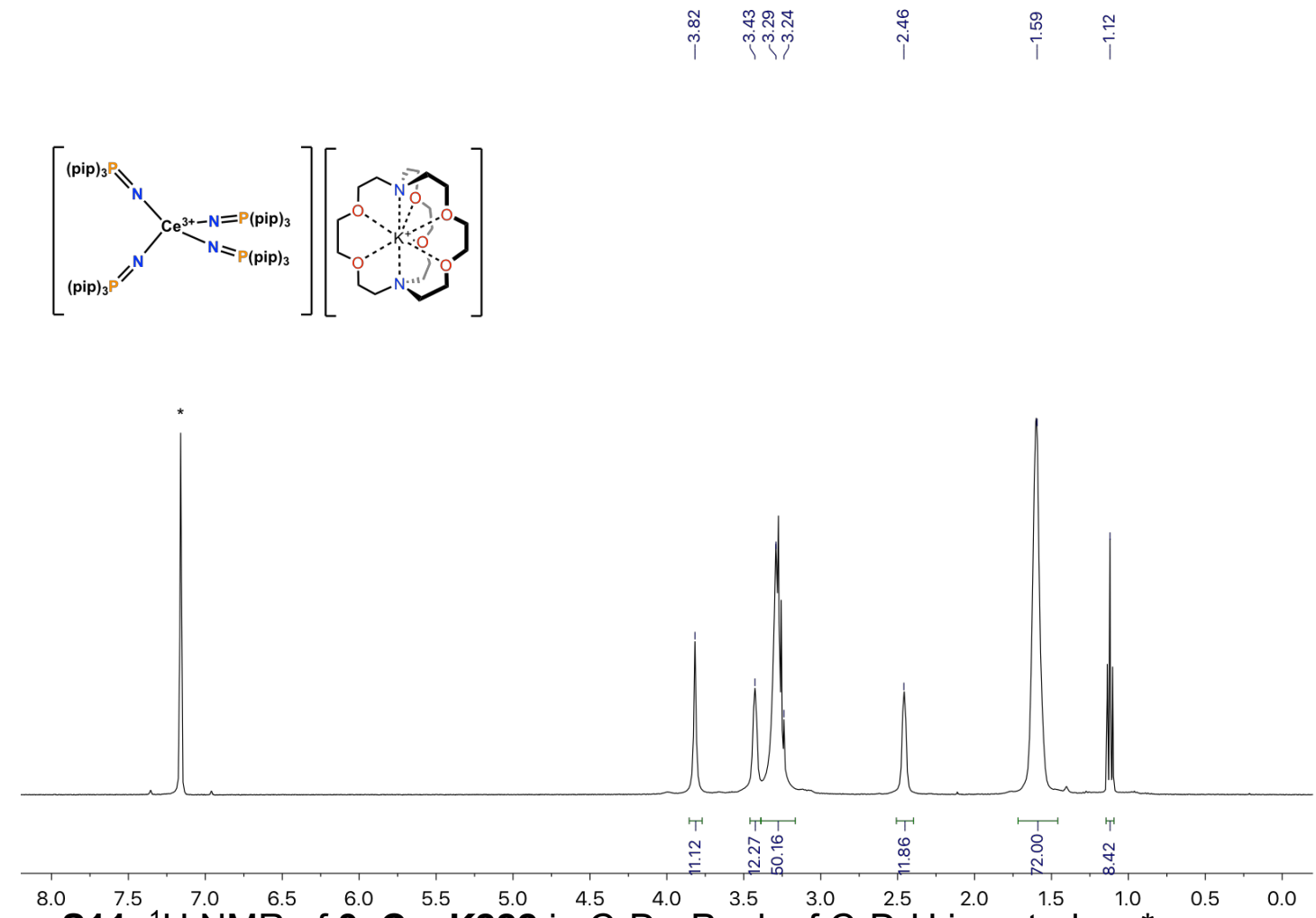

Figure S11. ${ }^{1} \mathrm{H}$ NMR of $3-\mathrm{Ce}-\mathrm{K} 222$ in $\mathrm{C}_{6} \mathrm{D}_{6}$. Peak of $\mathrm{C}_{6} \mathrm{D}_{5} \mathrm{H}$ is noted as * 


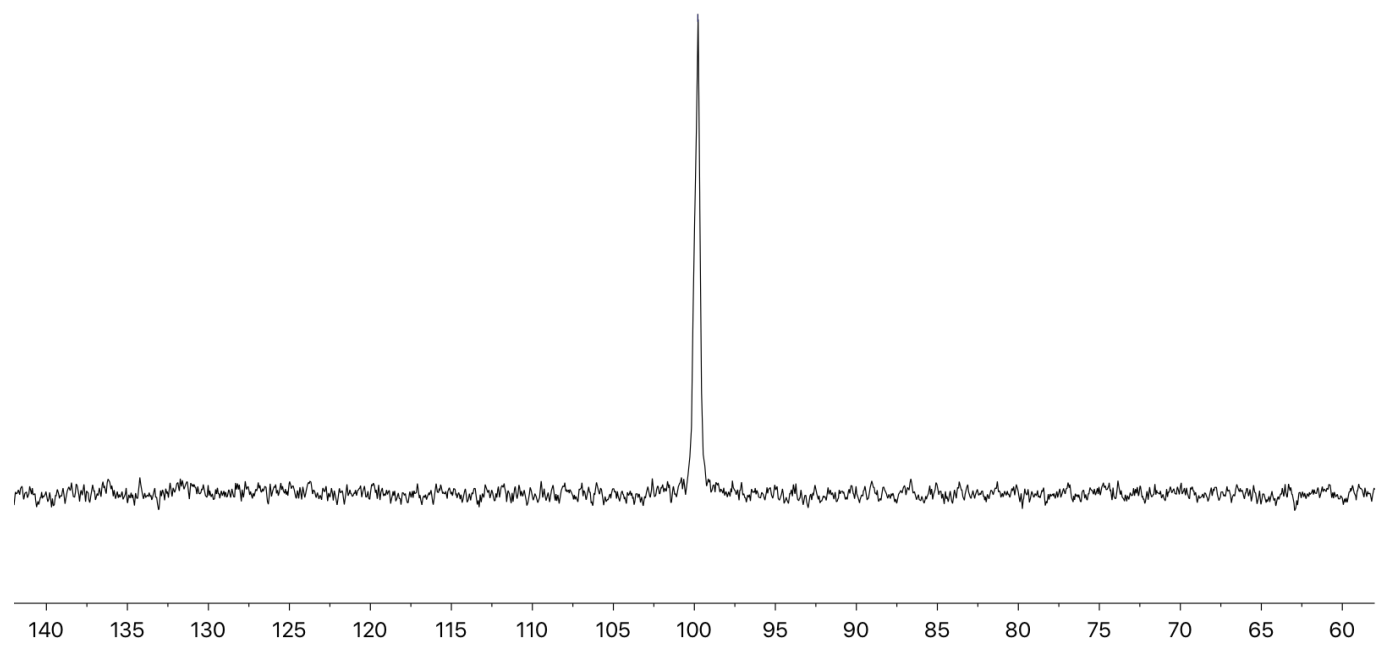

Figure S12. ${ }^{31} \mathrm{P}$ NMR of $3-\mathrm{Ce}-\mathrm{K} 222$ in $\mathrm{C}_{6} \mathrm{D}_{6}$.

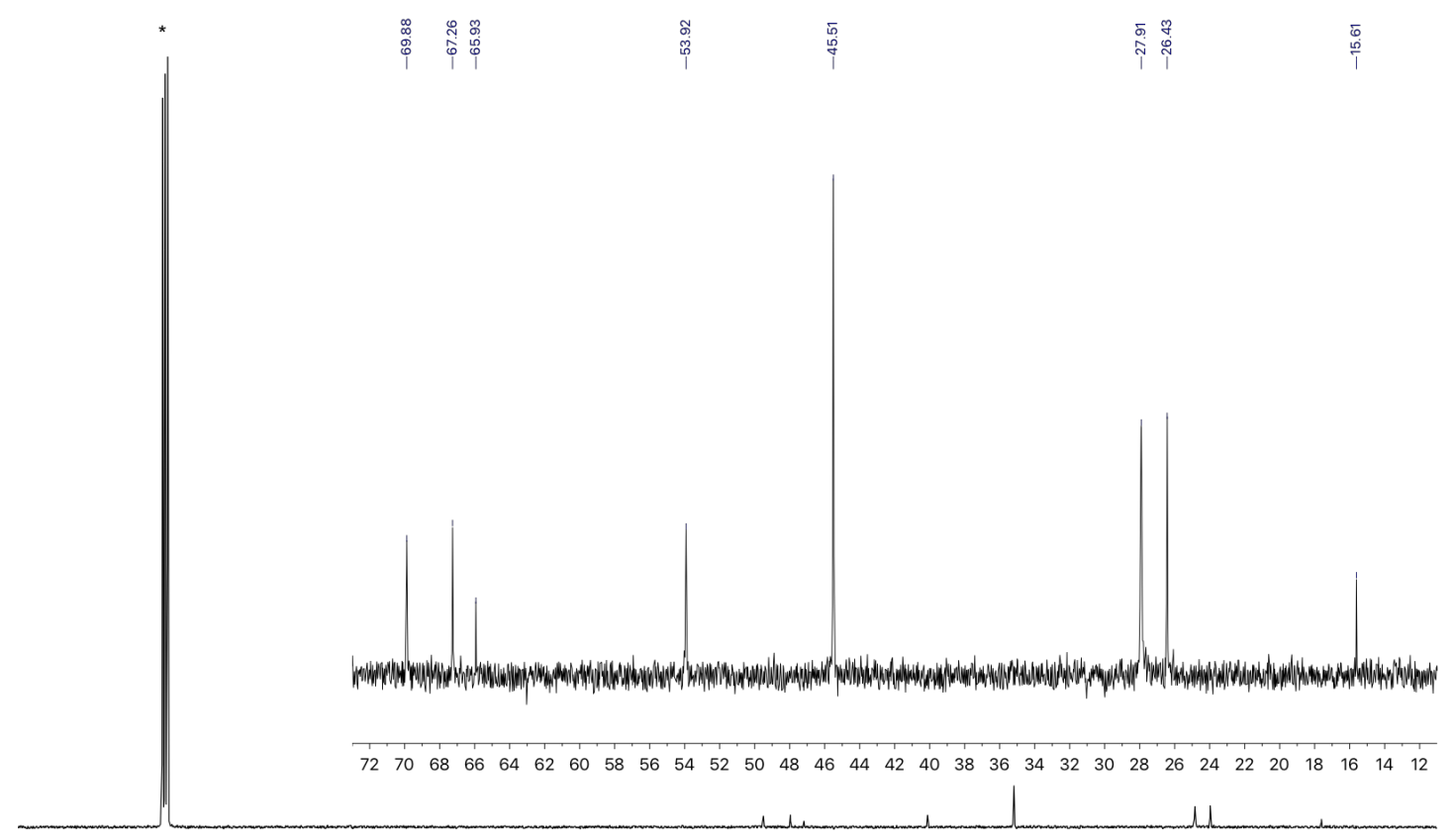

$\begin{array}{llllllllllllllllllllllllllll}140 & 135 & 130 & 125 & 120 & 115 & 110 & 105 & 100 & 95 & 90 & 85 & 80 & 75 & 70 & 65 & 60 & 55 & 50 & 45 & 40 & 35 & 30 & 25 & 20 & 15 & 10 & 5\end{array}$

Figure S13. ${ }^{13} \mathrm{C}$ NMR of 3-Ce-K222 in $\mathrm{C}_{6} \mathrm{D}_{6}$. Peak of $\mathrm{C}_{6} \mathrm{D}_{5} \mathrm{H}$ is noted as *. 


\section{Crystallographic Analyses}

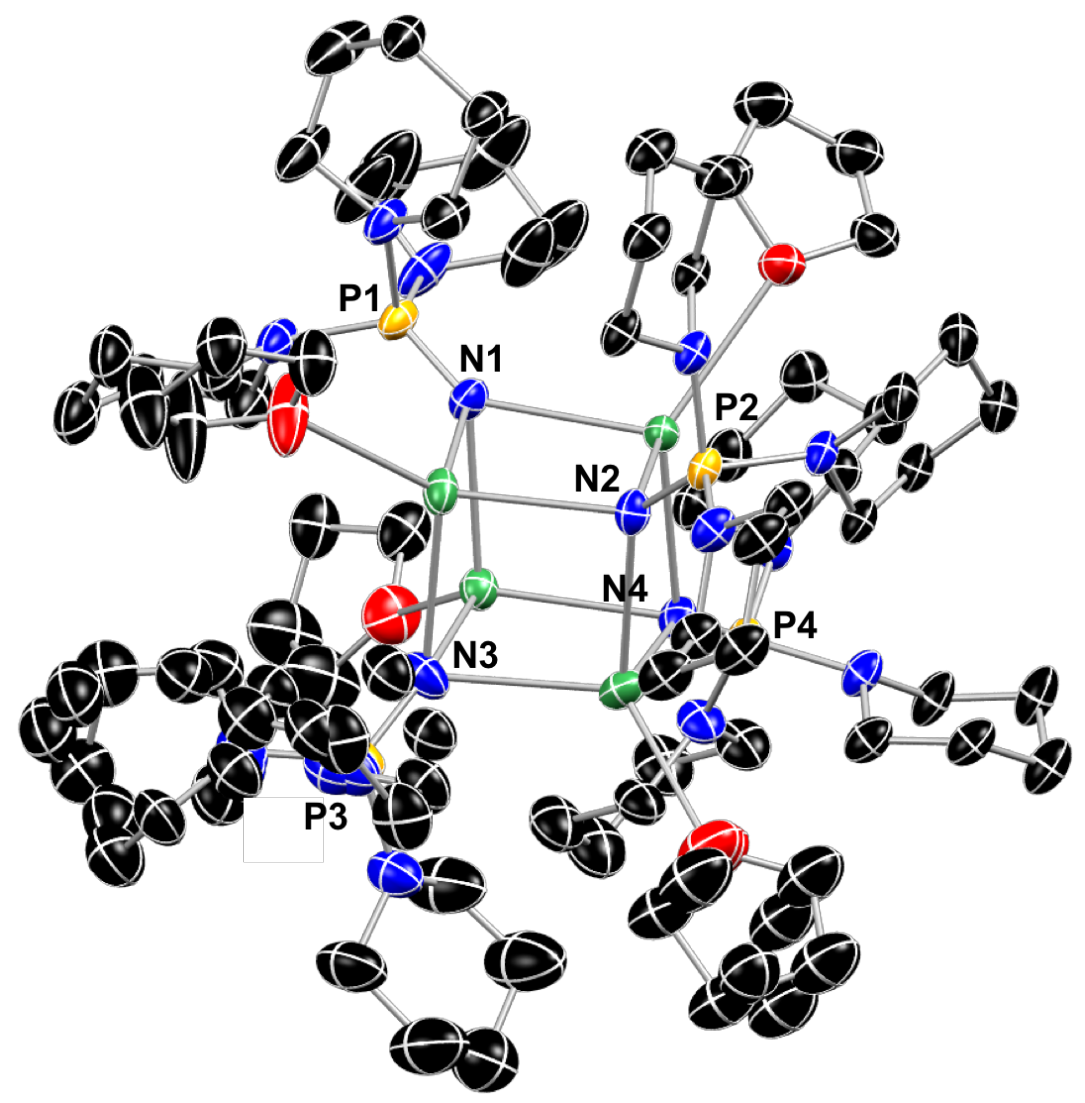

Figure S14. Molecular structure of $1-\mathrm{K}$ in the tetrameric heterocubane, $\mathrm{K}_{4}\left[\left(\mathrm{C}_{5} \mathrm{H}_{10} \mathrm{~N}\right)_{3} \mathrm{PN}\right]_{4} \bullet 4 \mathrm{THF}$, with thermal ellipsoids shown at $50 \%$ probability and $\mathrm{H}$ atoms are omitted for clarity. 


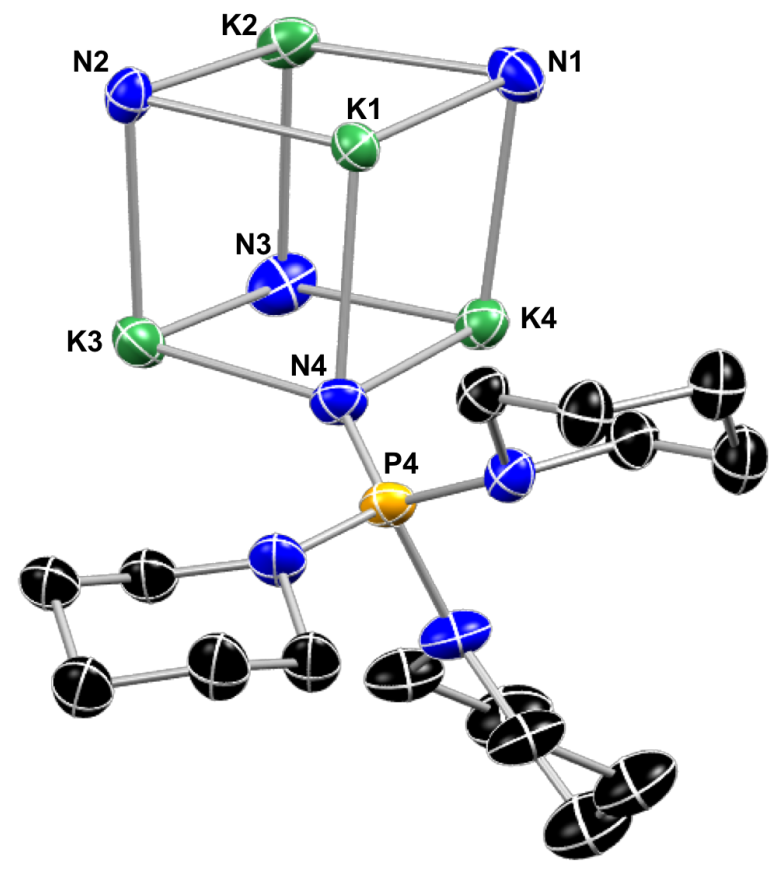

Figure S15. Molecular structure of $1-K$ with thermal ellipsoids shown at $50 \%$ probability. THF molecules capping potassium atoms, all but one ligand arm, and $\mathrm{H}$ atoms are omitted for clarity.

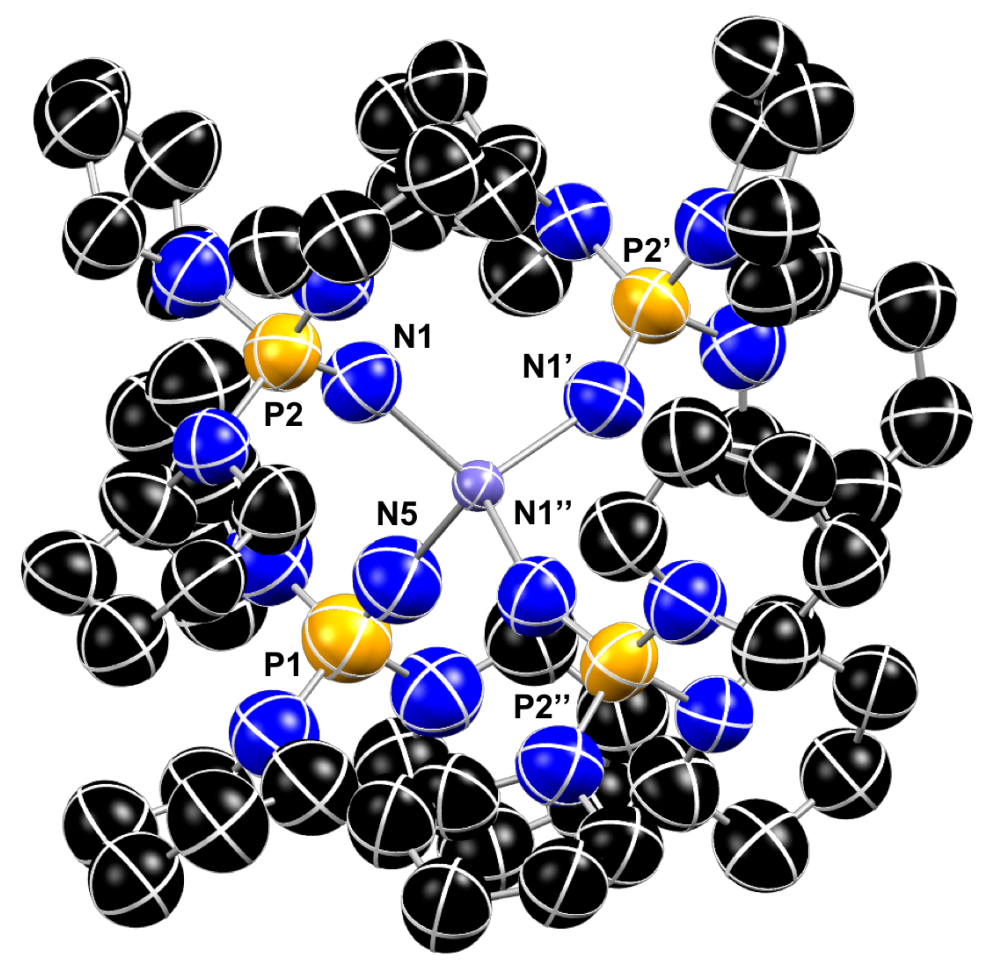

Figure S16. Molecular structure of 2-Ce with thermal ellipsoids shown at $50 \%$ probability and $\mathrm{H}$ atoms are omitted for clarity. 


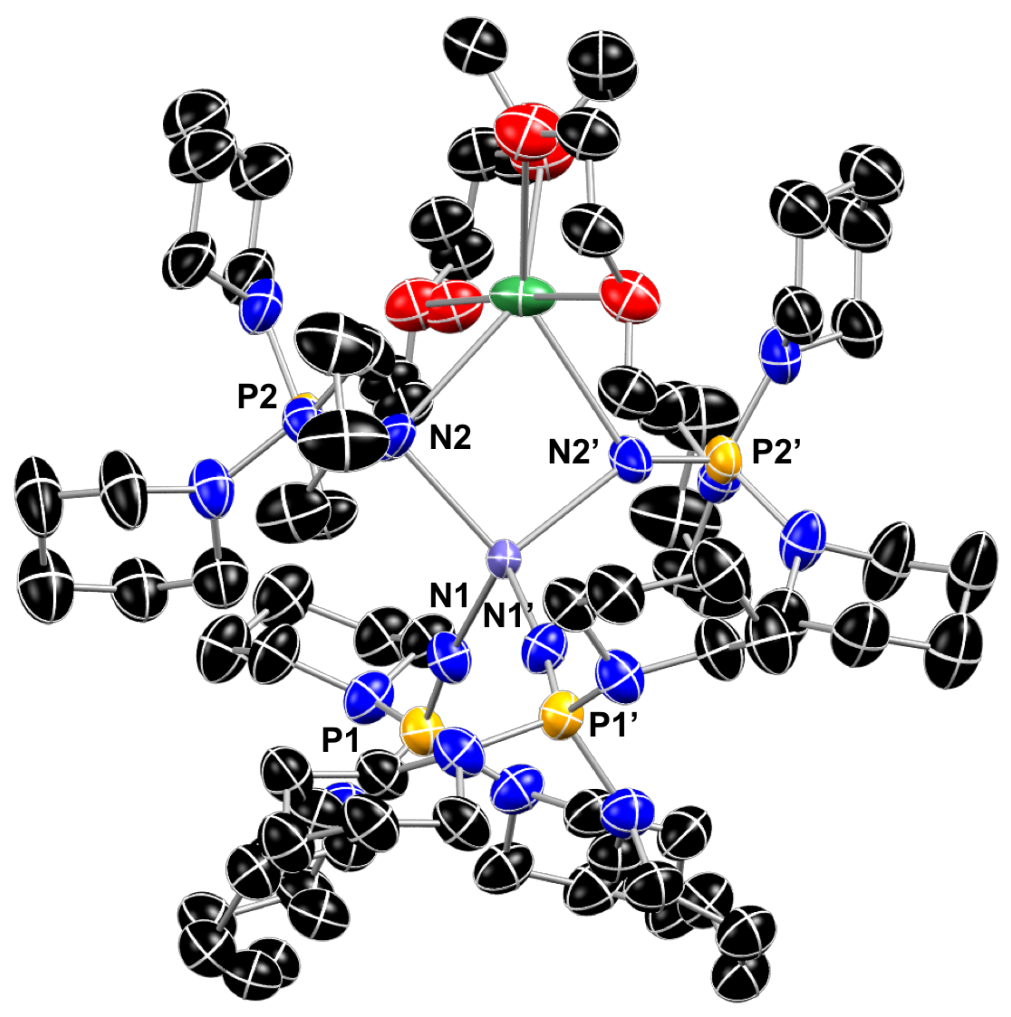

Figure S17. Molecular structure of 3-Ce-K(DME) 2 with thermal ellipsoids shown at $50 \%$ probability and $\mathrm{H}$ atoms are omitted for clarity. 


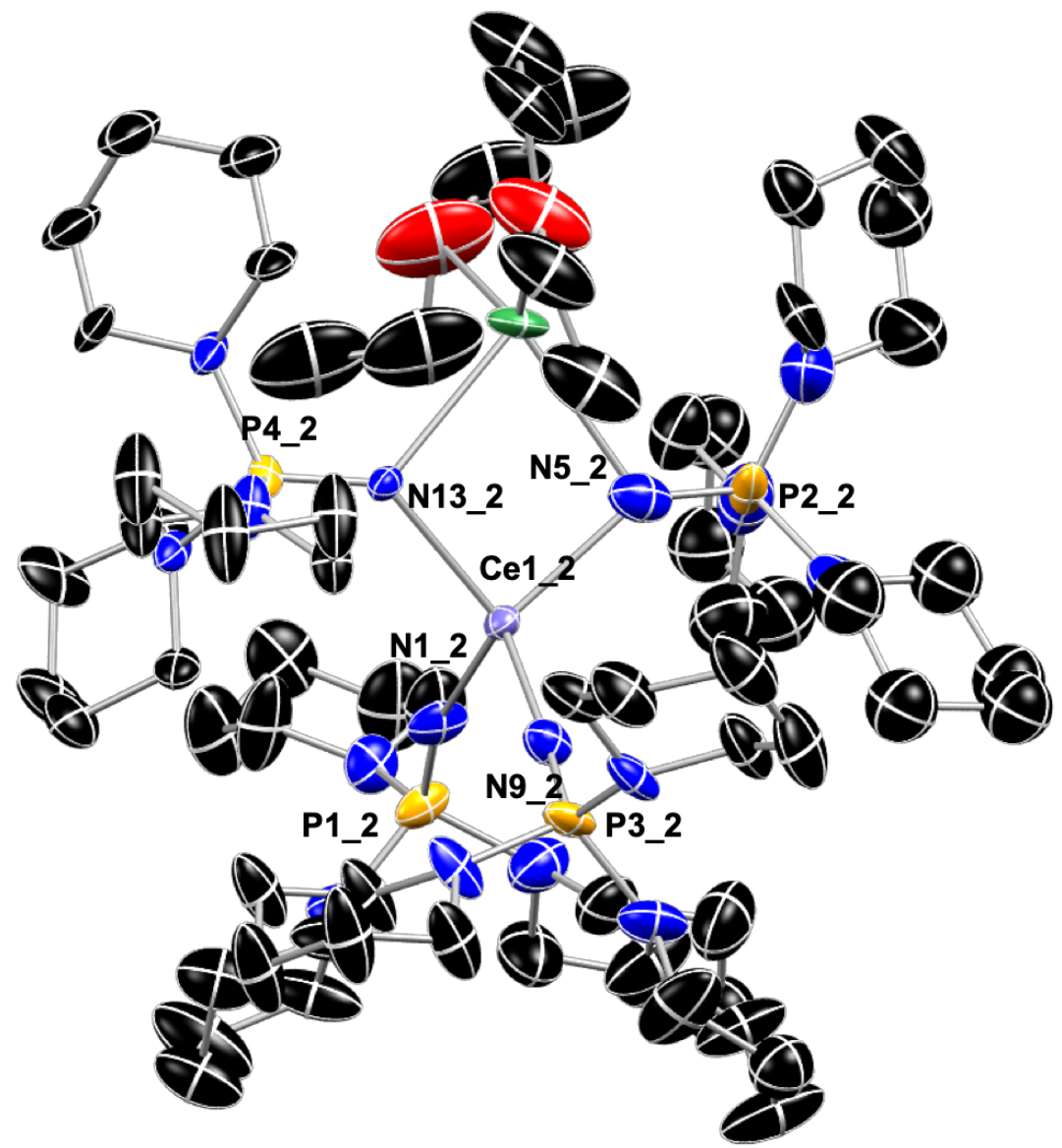

Figure S18. Molecular structure of $3-\mathrm{Ce}-\mathbf{K}\left(\mathrm{Et}_{2} \mathrm{O}\right)$ with thermal ellipsoids shown at $50 \%$ probability and $\mathrm{H}$ atoms are omitted for clarity. 


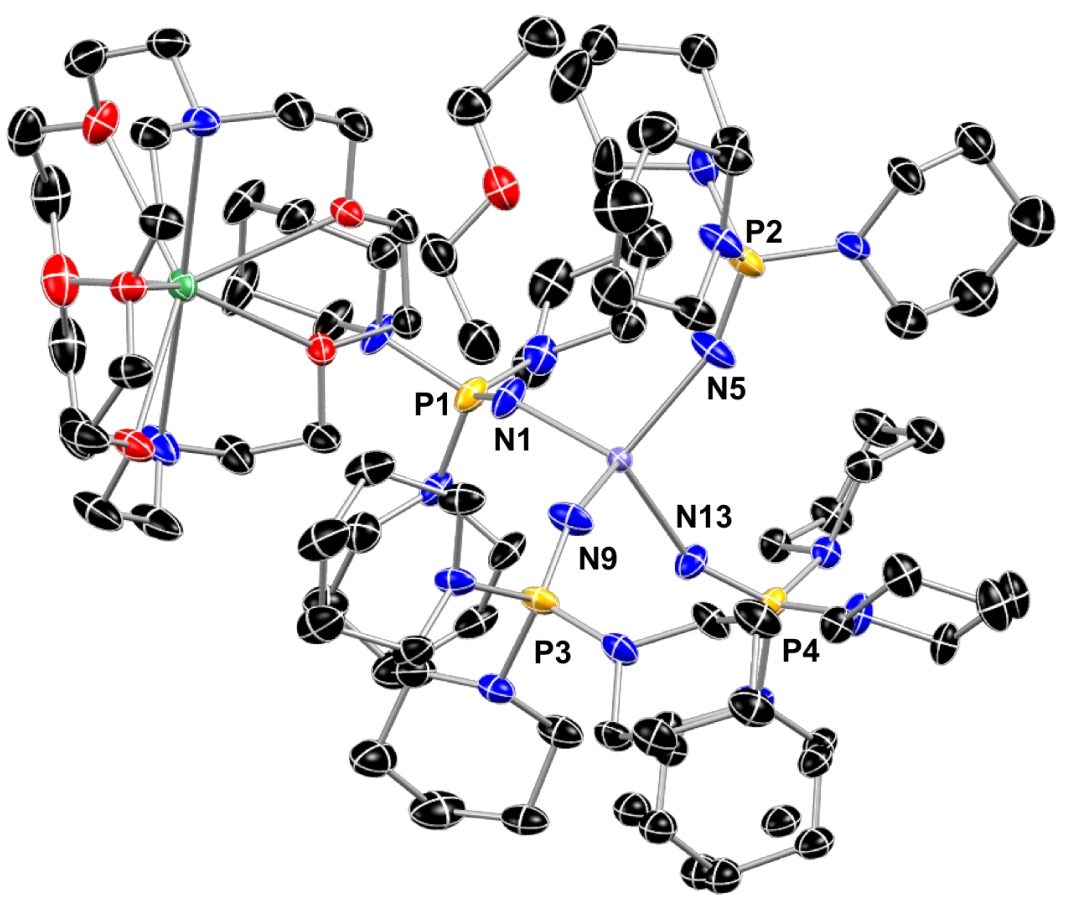

Figure S19. Molecular structure of 3-Ce-K222 with thermal ellipsoids shown at $50 \%$ probability and $\mathrm{H}$ atoms are omitted for clarity. 
Table S1. Crystallographic Data

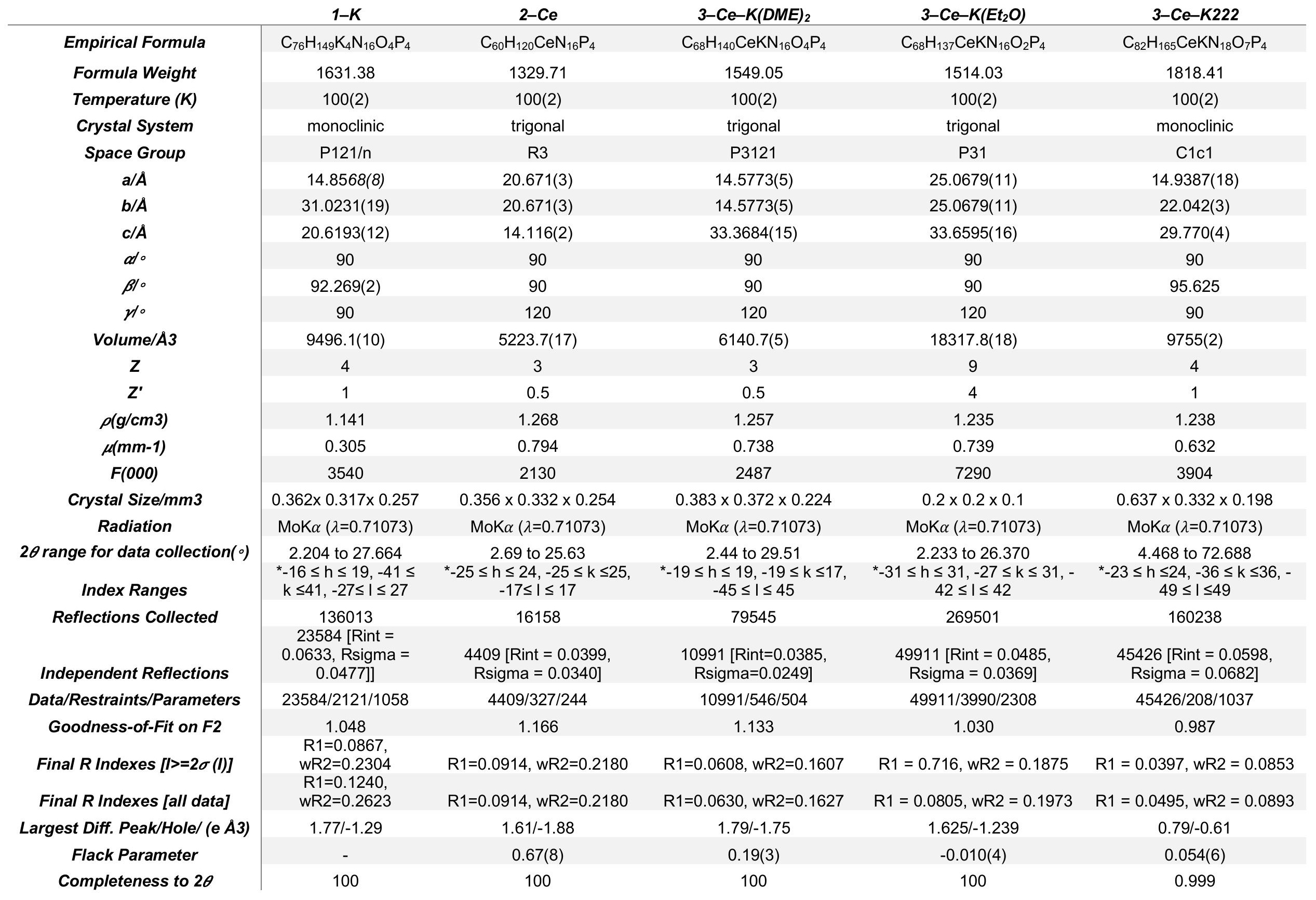


Table S2: Bond Lengths in $\AA$ for 1-K.

\begin{tabular}{lll}
\hline Atom & Atom & Length/A \\
\hline K1 & N1 & $2.727(3)$ \\
K1 & N4 & $2.738(3)$ \\
K1 & N2 & $2.767(3)$ \\
K1 & C6_11 & $3.489(4)$ \\
K1 & O1_14 & $2.871(3)$ \\
K4 & N1 & $2.735(4)$ \\
K4 & N4 & $2.754(3)$ \\
K4 & N3 & $2.676(4)$ \\
K4 & O1_15 & $2.704(3)$ \\
K3 & N4 & $2.690(3)$ \\
K3 & N3 & $2.732(4)$ \\
K3 & N2 & $2.719(3)$ \\
K3 & O1_16 & $2.784(3)$ \\
K3 & O1_17 & $2.782(3)$ \\
K2 & N1 & $2.766(4)$ \\
K2 & N3 & $2.716(4)$ \\
K2 & N2 & $2.712(3)$ \\
K2 & O1_18 & $2.751(3)$ \\
P3 & N3 & $1.518(4)$ \\
P3 & N5_4 & $1.699(3)$ \\
P3 & N5_5 & $1.694(3)$ \\
P3 & N5_6 & $1.698(3)$ \\
P3 & N5_7 & $1.708(3)$ \\
P3 & N5_19 & $1.705(3)$ \\
P4 & N4 & $1.522(3)$ \\
P4 & N5_8 & $1.708(3)$ \\
P4 & N5_9 & $1.710(3)$ \\
P4 & N5_10 & $1.717(3)$ \\
P1 & N1 & $1.517(3)$ \\
P1 & N5_1 & $1.699(3)$ \\
P1 & N5_2 & $1.710(3)$ \\
P1 & N5_3 & $1.700(3)$ \\
P2 & N2 & $1.516(3)$ \\
P2 & N5_11 & $1.715(2)$ \\
P2 & N5_12 & $1.712(2)$ \\
P2 & N5_13 & $1.705(2)$ \\
N5_1 & C2_1 & $1.4613(18)$ \\
N5_1 & C6_1 & $1.4584(18)$ \\
C2_1 & C3_1 & $1.516(2)$ \\
C3_1 & C4_1 & $1.521(2)$ \\
C4_1 & C5_1 & $1.523(2)$ \\
C5_1 & C6_1 & $1.503(2)$ \\
N5_2 & C2_2 & $1.4609(18)$ \\
N5_2 & C6_2 & $1.4596(17)$ \\
C2_2 & C3_2 & $1.514(2)$ \\
C3_2 & C4_2 & $1.518(2)$ \\
C4_2 & C5_2 & $1.521(2)$ \\
C5_2 & C6_2 & $1.504(2)$ \\
N5_3 & C2_3 & $1.4618(18)$ \\
N5_3 & C6_3 & $1.4619(18)$ \\
C2_3 & C3_3 & $1.518(2)$ \\
C3_3 & C4_3 & $1.520(2)$ \\
C4_3 & C5_3 & $1.523(2)$ \\
C5_3 & C6_3 & $1.507(2)$ \\
N5_4 & C2_4 & $1.4606(18)$ \\
& &
\end{tabular}

\begin{tabular}{lll} 
& & \\
\hline Atom & Atom & Length/A \\
\hline N5_4 & C6_4 & $1.4603(18)$ \\
C2_4 & C3_4 & $1.516(2)$ \\
C3_4 & C4_4 & $1.521(2)$ \\
C4_4 & C5_4 & $1.523(2)$ \\
C5_4 & C6_4 & $1.503(2)$ \\
N5_5 & C2_5 & $1.4617(18)$ \\
N5_5 & C6_5 & $1.4613(18)$ \\
C2_5 & C3_5 & $1.5183(16)$ \\
C3_5 & C4_5 & $1.5194(16)$ \\
C4_5 & C5_5 & $1.5212(16)$ \\
C5_5 & C6_5 & $1.507(2)$ \\
N5_6 & C2_6 & $1.4608(18)$ \\
N5_6 & C6_6 & $1.4617(18)$ \\
C2_6 & C3_6 & $1.517(2)$ \\
C3_6 & C4_6 & $1.521(2)$ \\
C4_6 & C5_6 & $1.524(2)$ \\
C5_6 & C6_6 & $1.507(2)$ \\
N5_7 & C2_7 & $1.4616(18)$ \\
N5_7 & C6_7 & $1.4618(18)$ \\
C2_7 & C3_7 & $1.516(2)$ \\
C3_7 & C4_7 & $1.520(2)$ \\
C4_7 & C5_7 & $1.523(2)$ \\
C5_7 & C6_7 & $1.506(2)$ \\
N5_8 & C2_8 & $1.4638(17)$ \\
N5_8 & C6_8 & $1.4632(17)$ \\
C2_8 & C3_8 & $1.518(2)$ \\
C3_8 & C4_8 & $1.519(2)$ \\
C4_8 & C5_8 & $1.522(2)$ \\
C5_8 & C6_8 & $1.506(2)$ \\
N5_9 & C2_9 & $1.4610(17)$ \\
N5_9 & C6_9 & $1.4607(17)$ \\
C2_9 & C3_9 & $1.516(2)$ \\
C3_9 & C4_9 & $1.520(2)$ \\
C4_9 & C5_9 & $1.523(2)$ \\
C5_9 & C6_9 & $1.506(2)$ \\
N5_10 & C2_10 & $1.4598(17)$ \\
N5_10 & C6_10 & $1.4609(17)$ \\
C2_10 & C3_10 & $1.514(2)$ \\
C3_10 & C4_10 & $1.520(2)$ \\
C4_10 & C5_10 & $1.525(2)$ \\
C5_10 & C6_10 & $1.507(2)$ \\
N5_11 & C2_11 & $1.4613(17)$ \\
N5_11 & C6_11 & $1.4613(17)$ \\
C2_11 & C3_11 & $1.517(2)$ \\
C3_11 & C4_11 & $1.521(2)$ \\
C4_11 & C5_11 & $1.522(2)$ \\
C5_11 & C6_11 & $1.507(2)$ \\
N5_12 & C2_12 & $1.4598(17)$ \\
N5_12 & C6_12 & $1.4612(17)$ \\
C2_12 & C3_12 & $1.516(2)$ \\
C3_12 & C4_12 & $1.520(2)$ \\
C4_12 & C5_12 & $1.524(2)$ \\
C5_12 & C6_12 & $1.507(2)$ \\
N5_13 & C2_13 & $1.4635(17)$ \\
N5_13 & C6_13 & $1.4631(17)$ \\
& &
\end{tabular}




\begin{tabular}{lll}
\hline Atom & Atom & Length/A \\
\hline C2_13 & C3_13 & $1.517(2)$ \\
C3_13 & C4_13 & $1.519(2)$ \\
C4_13 & C5_13 & $1.522(2)$ \\
C5_13 & C6_13 & $1.506(2)$ \\
N5_19 & C2_19 & $1.4614(18)$ \\
N5_19 & C6_19 & $1.4613(18)$ \\
C2_19 & C3_19 & $1.517(2)$ \\
C3_19 & C4_19 & $1.520(2)$ \\
C4_19 & C5_19 & $1.523(2)$ \\
C5_19 & C6_19 & $1.507(2)$ \\
O1_14 & C2_14 & $1.418(4)$ \\
O1_14 & C5_14 & $1.421(4)$ \\
C2_14 & C3_14 & $1.450(5)$ \\
C3_14 & C4_14 & $1.514(4)$ \\
C4_14 & C5_14 & $1.489(4)$ \\
O1_15 & C2_15 & $1.411(4)$ \\
O1_15 & C5_15 & $1.420(4)$ \\
C2_15 & C3_15 & $1.445(5)$ \\
C3_15 & C4_15 & $1.515(5)$ \\
C4_15 & C5_15 & $1.491(4)$ \\
O1_16 & C2_16 & $1.411(4)$ \\
O1_16 & C5_16 & $1.416(4)$ \\
C2_16 & C3_16 & $1.445(5)$ \\
C3_16 & C4_16 & $1.513(5)$ \\
C4_16 & C5_16 & $1.485(4)$ \\
O1_17 & C2_17 & $1.410(4)$ \\
O1_17 & C5_17 & $1.417(4)$ \\
C2_17 & C3_17 & $1.444(5)$ \\
C3_17 & C4_17 & $1.514(5)$ \\
C4_17 & C5_17 & $1.488(4)$ \\
O1_18 & C2_18 & $1.410(4)$ \\
O1_18 & C5_18 & $1.414(4)$ \\
C2_18 & C3_18 & $1.443(5)$ \\
C3_18 & C4_18 & $1.509(5)$ \\
C4_18 & C5_18 & $1.486(4)$ \\
& &
\end{tabular}


Table S3: Bond Angles in ${ }^{\circ}$ for $1-K$.

\begin{tabular}{|c|c|c|c|}
\hline Atom & Atom & Atom & ${\text { Angle } l^{\circ}}^{\circ}$ \\
\hline$\overline{\mathrm{N} 1}$ & K1 & N4 & $98.54(10)$ \\
\hline N1 & $\mathrm{K} 1$ & N2 & $97.15(10)$ \\
\hline N1 & K1 & C6_11 & 155.96(9) \\
\hline N1 & $\mathrm{K} 1$ & O1_14 & 111.87(10) \\
\hline N4 & K1 & $\mathrm{N} 2$ & $91.82(10)$ \\
\hline N4 & $\mathrm{K} 1$ & C6_11 & $81.32(8)$ \\
\hline N4 & K1 & O1_14 & $127.77(9)$ \\
\hline N2 & K1 & $\mathrm{C} 6^{-11}$ & $58.92(8)$ \\
\hline N2 & K1 & O1_14 & $123.37(10)$ \\
\hline 01_14 & $\mathrm{K} 1$ & C6_11 & 85.96(8) \\
\hline N1 & K4 & N4 & $97.96(10)$ \\
\hline N3 & K4 & N1 & $96.85(12)$ \\
\hline N3 & K4 & N4 & $95.37(11)$ \\
\hline N3 & K4 & O1_15 & $125.58(12)$ \\
\hline 01_15 & K4 & N1 & $113.05(11)$ \\
\hline 01_15 & K4 & N4 & 122.19(11) \\
\hline $\mathrm{N} 4$ & K3 & N3 & $95.57(11)$ \\
\hline N4 & K3 & N2 & $93.94(10)$ \\
\hline N4 & K3 & O1_16 & $121.1(2)$ \\
\hline N4 & K3 & O1_17 & $124.8(2)$ \\
\hline N3 & K3 & O1_16 & $125.0(3)$ \\
\hline N3 & K3 & O1_17 & $123.8(3)$ \\
\hline N2 & K3 & N3 ${ }^{-}$ & 93.83(10) \\
\hline N2 & K3 & O1_16 & $119.9(3)$ \\
\hline N2 & K3 & O1_17 & 117.2(3) \\
\hline N3 & K2 & N1 & $95.20(11)$ \\
\hline N3 & $\mathrm{K} 2$ & 01_18 & $119.66(12)$ \\
\hline N2 & K2 & $\mathrm{N} 1$ & $97.52(10)$ \\
\hline N2 & K2 & N3 & $94.33(11)$ \\
\hline N2 & K2 & O1_18 & $129.59(10)$ \\
\hline 01_18 & $\mathrm{K} 2$ & $\mathrm{~N} 1{ }^{-}$ & $113.55(13)$ \\
\hline K3 & P3 & K2 & $58.89(2)$ \\
\hline N3 & P3 & K3 & $41.00(16)$ \\
\hline N3 & P3 & K2 & $34.32(14)$ \\
\hline N3 & P3 & N5_4 & $113.8(3)$ \\
\hline N3 & P3 & N5_5 & $120.6(3)$ \\
\hline N3 & P3 & N5_6 & 115.8(2) \\
\hline N3 & P3 & N5_7 & $115.3(2)$ \\
\hline N3 & P3 & N5_19 & $120.8(4)$ \\
\hline N5_4 & P3 & $\mathrm{K} 3$ & 153.8(3) \\
\hline N5_4 & P3 & K2 & $105.1(3)$ \\
\hline N5_4 & P3 & N5_19 & $95.4(5)$ \\
\hline N5_5 & P3 & $\mathrm{K} 3$ & $160.6(3)$ \\
\hline N5_5 & P3 & K2 & $110.4(3)$ \\
\hline N5_5 & P3 & N5_6 & $99.9(3)$ \\
\hline N5_5 & P3 & N5_7 & $100.7(3)$ \\
\hline N5_6 & P3 & $\mathrm{K} 3$ & $87.66(14)$ \\
\hline N5_6 6 & P3 & K2 & $146.12(14)$ \\
\hline N5_6 & P3 & N5_4 & $103.6(4)$ \\
\hline N5_6 & P3 & N5_7 & 101.6(3) \\
\hline N5_6 & P3 & N5_19 & $104.4(3)$ \\
\hline N5_7 & P3 & K3 & $95.27(19)$ \\
\hline N5_7 & P3 & K2 & $87.8(2)$ \\
\hline N5 19 & P3 & K3 & $104.6(3)$ \\
\hline N5_19 & P3 & K2 & $90.3(3)$ \\
\hline
\end{tabular}

\begin{tabular}{|c|c|c|c|}
\hline Atom & Atom & Atom & Angle $^{\circ}$ \\
\hline$\overline{\mathrm{K} 4}$ & $\mathrm{P} 4$ & $\mathrm{~K} 1$ & $56.62(2)$ \\
\hline N4 & P4 & $\mathrm{K} 1$ & $37.98(13)$ \\
\hline N4 & P4 & $\mathrm{K} 4$ & $38.83(13)$ \\
\hline N4 & P4 & N5 8 & $121.69(17)$ \\
\hline N4 & P4 & N5 9 & $115.10(17)$ \\
\hline N4 & $\mathrm{P} 4$ & N5_10 & $114.76(16)$ \\
\hline N5_8 & $\mathrm{P} 4$ & $\mathrm{~K}^{-}$ & $87.09(10)$ \\
\hline N5_8 & $\mathrm{P} 4$ & $\mathrm{~K} 4$ & $104.20(10)$ \\
\hline N5_8 & P4 & N5_9 & $96.97(14)$ \\
\hline N5_8 & P4 & N5_10 & $98.12(13)$ \\
\hline N5_9 & $\mathrm{P} 4$ & $\mathrm{~K}^{-}$ & $141.72(12)$ \\
\hline N5_9 & $\mathrm{P} 4$ & $\mathrm{~K} 4$ & $85.70(11)$ \\
\hline N5 9 & P4 & N5_10 & $107.52(17)$ \\
\hline N5_10 & P4 & $\mathrm{K} 1$ & $109.54(11)$ \\
\hline N5_10 & P4 & K4 & $152.50(9)$ \\
\hline $\mathrm{K} 2^{-}$ & $\mathrm{P} 1$ & $\mathrm{~K} 4$ & $56.83(2)$ \\
\hline N1 & $\mathrm{P} 1$ & K4 & $34.60(14)$ \\
\hline N1 & P1 & K2 & $39.26(15)$ \\
\hline N1 & $\mathrm{P} 1$ & N5_1 & $115.05(19)$ \\
\hline N1 & $\mathrm{P} 1$ & $\mathrm{~N} 5-2$ & $115.48(17)$ \\
\hline N1 & $\mathrm{P} 1$ & N5_3 & $122.88(19)$ \\
\hline N5_1 & P1 & $\mathrm{K} 4$ & $102.59(12)$ \\
\hline N5_1 & P1 & K2 & $154.31(11)$ \\
\hline $\mathrm{N} 5^{-} 1$ & $\mathrm{P} 1$ & N5 2 & $105.01(18)$ \\
\hline N5 1 & $\mathrm{P} 1$ & N5_3 & $97.82(17)$ \\
\hline N5_2 & P1 & $\mathrm{K} 4$ & $147.33(11)$ \\
\hline N5_2 & $\mathrm{P} 1$ & K2 & $91.50(12)$ \\
\hline N5 3 & $\mathrm{P} 1$ & K4 & $95.78(12)$ \\
\hline N5_3 & $\mathrm{P} 1$ & K2 & $99.41(13)$ \\
\hline N5_3 & $\mathrm{P} 1$ & N5_2 & $97.44(15)$ \\
\hline $\mathrm{N} 2^{-}$ & $\mathrm{P} 2$ & $\mathrm{~K}^{-}{ }^{-}$ & $45.81(12)$ \\
\hline N2 & $\mathrm{P} 2$ & N5 11 & $114.12(15)$ \\
\hline N2 & $\mathrm{P} 2$ & N5_12 & $115.36(16)$ \\
\hline N2 & P2 & N5_13 & $122.33(16)$ \\
\hline N5_11 & P2 & $\mathrm{K} 1$ & $77.46(10)$ \\
\hline N5_12 & $\mathrm{P} 2$ & K1 & $158.09(10)$ \\
\hline N5_12 & $\mathrm{P} 2$ & N5_11 & $108.26(15)$ \\
\hline N5_13 & P2 & K1 & $102.70(9)$ \\
\hline $\mathrm{N} 5-13$ & P2 & N5 11 & $96.36(13)$ \\
\hline N5_13 & $\mathrm{P} 2$ & N5_12 & $97.72(13)$ \\
\hline $\mathrm{K} 1$ & N1 & K4 & $81.79(9)$ \\
\hline $\mathrm{K} 1$ & N1 & $\mathrm{K} 2$ & $82.36(10)$ \\
\hline K4 & N1 & K2 & $82.40(10)$ \\
\hline P1 & N1 & $\mathrm{K} 1$ & $142.8(2)$ \\
\hline P1 & N1 & $\mathrm{K} 4$ & $127.04(19)$ \\
\hline P1 & N1 & K2 & $120.43(19)$ \\
\hline $\mathrm{K} 1$ & N4 & K4 & $81.26(9)$ \\
\hline K3 & N4 & $\mathrm{K} 1$ & 87.12(9) \\
\hline K3 & N4 & K4 & $84.00(9)$ \\
\hline P4 & N4 & $\mathrm{K} 1$ & $122.03(18)$ \\
\hline P4 & N4 & K4 & $120.91(17)$ \\
\hline P4 & N4 & K3 & $142.27(19)$ \\
\hline K4 & N3 & K3 & $84.68(10)$ \\
\hline K4 & N3 & $\mathrm{K} 2$ & $84.44(11)$ \\
\hline $\mathrm{K} 2$ & N3 & K3 & $85.54(10)$ \\
\hline
\end{tabular}




\begin{tabular}{|c|c|c|c|}
\hline Atom & Atom & Atom & Angle $^{\circ}$ \\
\hline$\overline{\mathrm{P} 3}$ & N3 & $\mathrm{K} 4$ & $140.0(2)$ \\
\hline P3 & N3 & K3 & $117.6(2)$ \\
\hline P3 & N3 & K2 & $127.3(2)$ \\
\hline K3 & N2 & K1 & $85.98(9)$ \\
\hline K2 & N2 & $\mathrm{K} 1$ & $82.62(9)$ \\
\hline K2 & N2 & K3 & $85.88(9)$ \\
\hline P2 & N2 & $\mathrm{K} 1$ & $111.07(16)$ \\
\hline P2 & N2 & K3 & $132.79(17)$ \\
\hline P2 & N2 & K2 & $138.12(18)$ \\
\hline C2_1 & N5_1 & P1 & $120.0(2)$ \\
\hline C6_1 & N5_1 & $\mathrm{P} 1$ & $112.6(2)$ \\
\hline $\mathrm{C} 6-1$ & $\mathrm{~N} 5-1$ & C2 1 & 111.7(2) \\
\hline N5_1 & $\mathrm{C} 2 \_1$ & C3_1 & $110.5(2)$ \\
\hline C2_1 & C3_1 & C4_1 & $110.8(2)$ \\
\hline $\mathrm{C} 3{ }^{-1}$ & $\mathrm{C} 4^{-} 1$ & $\mathrm{C} 5^{-} 1$ & 109.7(2) \\
\hline C6_1 & C5_1 & C4_1 & $111.4(2)$ \\
\hline N5_-1 & C6_1 & C5_1 & 112.1(2) \\
\hline C2_2 & N5_2 & $\mathrm{P} 1^{-}$ & $126.97(19)$ \\
\hline $\mathrm{C} 6{ }^{-} 2$ & N5 2 & P1 & $119.40(19)$ \\
\hline C6 62 & N5 2 & C2 2 & $111.2(2)$ \\
\hline N5_2 & C2_2 & C3_2 & $111.3(2)$ \\
\hline C2_2 & C3_2 & C4_2 & $111.4(2)$ \\
\hline $\mathrm{C} 3{ }^{-} 2$ & $\mathrm{C} 4{ }^{-} 2$ & $\mathrm{C} 5{ }^{-} 2$ & $110.2(2)$ \\
\hline C6_2 & C5_2 & C4_2 & $111.6(2)$ \\
\hline N5_2 & C6_2 & C5_2 & $111.1(2)$ \\
\hline $\mathrm{C} 23$ & $\mathrm{~N} 5^{-} 3$ & $\mathrm{P}^{-}$ & $115.2(2)$ \\
\hline $\mathrm{C} 2-3$ & N5_3 & C6_3 & 111.2(2) \\
\hline C6_3 & N5_3 & $\mathrm{P} 1{ }^{-}$ & $118.1(2)$ \\
\hline N5_3 & C2_3 & C3_3 & $110.5(2)$ \\
\hline $\mathrm{C} 23$ & $\mathrm{C} 3^{-} 3$ & $\mathrm{C} 4-3$ & $111.0(2)$ \\
\hline $\mathrm{C} 3-3$ & $\mathrm{C} 4{ }^{-} 3$ & $\mathrm{C} 5$ & $110.1(2)$ \\
\hline C6_3 & C5_3 & C4_3 & $111.1(2)$ \\
\hline N5_3 & C6_3 & C5_3 & $110.7(2)$ \\
\hline $\mathrm{C} 2-4$ & $\mathrm{~N} 5^{-} 4$ & $\mathrm{P}^{-}$ & $129.2(5)$ \\
\hline C6 64 & $\mathrm{~N} 5$ & P3 & $118.9(5)$ \\
\hline C6_4 & N5_4 & C2_4 & $111.9(2)$ \\
\hline N5_4 & C2_4 & C3_4 & $111.0(3)$ \\
\hline $\mathrm{C} 2-4$ & $\mathrm{C}^{-} 4$ & $\mathrm{C} 4-4$ & $110.8(2)$ \\
\hline C3_4 & C4_4 & C5_4 & $109.5(2)$ \\
\hline C6_4 & C5_4 & C4_4 & 111.3(3) \\
\hline N5_4 & C6_4 & C5_4 & $112.0(2)$ \\
\hline $\mathrm{C} 25$ & N5 5 & $\mathrm{P} 3$ & $115.2(5)$ \\
\hline C6_5 & N5_5 & P3 & $114.2(4)$ \\
\hline C6_5 & N5_5 & C2_5 & 111.3(2) \\
\hline N5_5 & C2_5 & C3_5 & 110.2(2) \\
\hline $\mathrm{C} 25$ & $\mathrm{C} 35$ & $\mathrm{C} 45$ & $111.0(2)$ \\
\hline C3_5 & C4_5 & C5_5 & $110.4(2)$ \\
\hline C6_5 & C5_5 & C4_5 & $111.4(2)$ \\
\hline $\mathrm{N} 55$ & $\mathrm{C} 65$ & $\mathrm{C} 55$ & 110.7(2) \\
\hline $\mathrm{C} 2 \_6$ & N5_6 & $\mathrm{P} 3$ & $126.7(2)$ \\
\hline C2_6 & N5_6 & C6_6 & $111.2(2)$ \\
\hline C6_6 & N5_6 & P3 & $117.6(2)$ \\
\hline N5_6 & C2_6 & C3_6 & $110.9(2)$ \\
\hline $\mathrm{C} 2 \_6$ & C3 6 & C4_6 & $111.0(2)$ \\
\hline C3_6 & C4_6 & C5_6 & $109.4(2)$ \\
\hline C6_6 & C5_6 & C4_6 & $110.6(2)$ \\
\hline N5_6 & C6_6 & C5_6 & $111.1(2)$ \\
\hline $\mathrm{C} 2 \_7$ & N5_7 & P3 & $126.3(3)$ \\
\hline C2_-7 & N5_7 & C6_7 & $111.3(2)$ \\
\hline
\end{tabular}

\begin{tabular}{|c|c|c|c|}
\hline Atom & Atom & Atom & Angle $^{\circ}$ \\
\hline$\overline{C 67}$ & N5 7 & P3 & $121.5(3)$ \\
\hline N5_7 & $\mathrm{C} 2 \_7$ & C3_7 & $111.1(2)$ \\
\hline $\mathrm{C} 2-7$ & C3_7 & C4_7 & $111.2(2)$ \\
\hline C3_7 & C4_7 & C5_7 & $109.8(2)$ \\
\hline C6_7 & C5_7 & C4_7 & $111.1(2)$ \\
\hline N5_7 & C6_7 & C5_7 & $111.1(2)$ \\
\hline C2_8 & N5_8 & $\mathrm{P}^{-}$ & $117.98(18)$ \\
\hline C6_8 & N5_8 & P4 & 115.71(18) \\
\hline C6_8 & N5_8 & C2_8 & $110.57(19)$ \\
\hline N5_8 & C2_8 & C3_8 & 109.96(19) \\
\hline C2_8 & C3_8 & C4_8 & 111.1(2) \\
\hline C3_8 & C4_8 & C5_8 & $110.5(2)$ \\
\hline C6_8 & C5_8 & C4_8 & $111.3(2)$ \\
\hline N5_8 & C6_8 & C5_8 & $110.68(19)$ \\
\hline C2_9 & N5_9 & $\mathrm{P}^{-}$ & $126.5(2)$ \\
\hline C6 9 & N5_9 & P4 & 118.2(2) \\
\hline C6_9 & N5_9 & C2_9 & 111.6(2) \\
\hline N5_9 & C2_9 & C3_9 & $111.0(2)$ \\
\hline C2_9 & C3_9 & C4_9 & 111.1(2) \\
\hline $\mathrm{C} 3-9$ & $\mathrm{C} 4-9$ & $\mathrm{C} 5$ & $109.9(2)$ \\
\hline C6_9 & C5_9 & C4_9 & $111.1(2)$ \\
\hline N5_9 & C6_9 & C5_9 & 111.1(2) \\
\hline C2_10 & N5_10 & P4 & 114.87(19) \\
\hline C2_10 & N5_10 & C6_10 & $111.9(2)$ \\
\hline C6_10 & N5_10 & $\mathrm{P} 4^{-}$ & $120.24(19)$ \\
\hline N5_10 & C2_10 & C3_10 & 111.7(2) \\
\hline C2_10 & C3_10 & C4_10 & $111.4(2)$ \\
\hline C3_10 & C4_10 & C5_10 & $109.4(2)$ \\
\hline C6_10 & C5_10 & C4_10 & $110.4(2)$ \\
\hline N5_10 & C6_10 & C5_10 & $110.8(2)$ \\
\hline C2_11 & N5_11 & $\mathrm{P} 2^{-}$ & $126.12(17)$ \\
\hline C2_11 & N5_11 & C6_11 & $111.68(19)$ \\
\hline C6_11 & N5_11 & $\mathrm{P} 2$ & $119.53(17)$ \\
\hline N5_11 & C2_11 & C3_11 & $110.68(19)$ \\
\hline C2_11 & C3_11 & C4_11 & $110.8(2)$ \\
\hline C3_11 & C4_11 & C5_11 & $109.99(19)$ \\
\hline C6_11 & C5_11 & C4_11 & $111.2(2)$ \\
\hline N5_11 & C6_11 & $\mathrm{K} 1^{-}$ & $83.91(17)$ \\
\hline N5_11 & C6_11 & C5_11 & $111.27(19)$ \\
\hline C5_11 & C6_11 & $\mathrm{K} 1$ & $81.16(17)$ \\
\hline C2_12 & N5_12 & P2 & $115.90(18)$ \\
\hline C2_12 & N5_12 & C6_12 & $111.9(2)$ \\
\hline C6_12 & N5_12 & $\mathrm{P} 2$ & $121.09(19)$ \\
\hline N5_12 & C2_12 & C3_12 & 111.1(2) \\
\hline C2_12 & C3_12 & C4_12 & $111.0(2)$ \\
\hline C3_12 & C4_12 & C5_12 & 109.6(2) \\
\hline C6_12 & C5_12 & C4_12 & $110.9(2)$ \\
\hline N5_12 & C6_12 & C5_12 & 111.2(2) \\
\hline C2_13 & N5_13 & $\mathrm{P} 2$ & $120.43(18)$ \\
\hline C6_13 & N5_13 & P2 & $115.22(17)$ \\
\hline C6_13 & N5_13 & C2_13 & $110.60(18)$ \\
\hline N5_13 & C2_13 & C3_13 & $110.07(19)$ \\
\hline C2_13 & C3_13 & C4_13 & $110.9(2)$ \\
\hline C3 13 & C4_13 & C5_13 & $110.4(2)$ \\
\hline C6_13 & C5_13 & C4_13 & 111.5(2) \\
\hline N5_13 & C6_13 & C5_13 & 110.93(19) \\
\hline C2_19 & N5_19 & $\mathrm{P}^{-}$ & $118.7(6)$ \\
\hline C6_19 & N5_19 & P3 & $107.3(5)$ \\
\hline C6_19 & N5_19 & C2_19 & $111.3(3)$ \\
\hline
\end{tabular}




\begin{tabular}{llll}
\hline Atom & Atom & Atom & Anglel $^{\circ}$ \\
\hline N5_19 & C2_19 & C3_19 & $110.5(2)$ \\
C2_19 & C3_19 & C4_19 & $111.0(3)$ \\
C3_19 & C4_19 & C5_19 & $110.0(3)$ \\
C6_19 & C5_19 & C4_19 & $111.1(3)$ \\
N5_19 & C6_19 & C5_19 & $110.8(3)$ \\
C2_14 & O1_14 & K1 & $142.9(2)$ \\
C2_14 & O1_14 & C5_14 & $106.5(2)$ \\
C5_14 & O1_14 & K1 & $109.9(2)$ \\
O1_14 & C2_14 & C3_14 & $105.7(3)$ \\
C2_14 & C3_14 & C4_14 & $105.2(3)$ \\
C5_14 & C4_14 & C3_14 & $103.9(3)$ \\
O1_14 & C5_14 & C4_14 & $107.3(3)$ \\
C2_15 & O1_15 & K4- & $138.1(3)$ \\
C2_15 & O1_15 & C5_15 & $109.0(2)$ \\
C5_15 & O1_15 & K4 & $111.7(2)$ \\
O1_15 & C2_15 & C3_15 & $108.5(3)$ \\
C2_15 & C3_15 & C4_15 & $105.5(3)$ \\
C5_15 & C4_15 & C3_15 & $101.9(3)$ \\
O1_15 & C5_15 & K4 & $46.11(17)$ \\
O1_15 & C5_15 & C4_15 & $106.4(3)$ \\
C4_15 & C5_15 & K4 & $141.8(3)$ \\
C2_16 & O1_16 & K3 & $130.5(5)$ \\
C2_16 & O1_16 & C5_16 & $109.4(3)$ \\
C5_16 & O1_16 & K3 & $120.0(5)$ \\
O1_16 & C2_16 & C3_16 & $107.7(3)$ \\
C2_16 & C3_16 & C4_16 & $105.5(3)$ \\
C5_16 & C4_16 & C3_16 & $103.4(3)$ \\
O1_16 & C5_16 & C4_16 & $108.4(3)$ \\
C2_17 & O1_17 & K3 & $126.4(5)$ \\
C2_17 & O1_17 & C5_17 & $109.4(3)$ \\
C5_17 & O1_17 & K3 & $124.1(5)$ \\
O1_17 & C2_17 & C3_17 & $108.6(3)$ \\
C2_17 & C3_17 & C4_17 & $106.1(3)$ \\
C5_17 & C4_17 & C3_17 & $102.6(3)$ \\
O1_17 & C5_17 & C4_17 & $107.0(3)$ \\
C2_18 & O1_18 & K2 & $133.1(3)$ \\
C2_18 & O1_18 & C5_18 & $109.4(2)$ \\
C5_18 & O1_18 & K2- & $116.9(2)$ \\
O1_18 & C2_18 & C3_18 & $108.0(3)$ \\
C2_18 & C3_18 & C4_18 & $106.0(3)$ \\
C5_18 & C4_18 & C3_18 & $103.9(3)$ \\
O1_18 & C5_18 & C4_18 & $108.5(3)$ \\
& & &
\end{tabular}


Table S4: Bond Lengths in $\AA$ for 2-Ce.

\begin{tabular}{|c|c|c|}
\hline Atom & Atom & Length/Å \\
\hline$\overline{\mathrm{Ce} 1}$ & $\mathrm{~N} 1^{1}$ & $2.17(2)$ \\
\hline Ce1 & N1 & $2.17(2)$ \\
\hline $\mathrm{Ce} 1$ & $\mathrm{~N} 1^{2}$ & 2.17(2) \\
\hline Ce1 & N5 & $2.27(6)$ \\
\hline $\mathrm{N} 1$ & $\mathrm{P} 2$ & $1.48(2)$ \\
\hline N5 & P1 & $1.42(6)$ \\
\hline P1 & N2_32 & $1.693(3)$ \\
\hline P1 & N2_3 & $1.693(3)$ \\
\hline $\mathrm{P} 1$ & $\mathrm{~N} 2{ }^{-} 3^{1}$ & $1.693(3)$ \\
\hline P2 & N2_1 & $1.696(2)$ \\
\hline P2 & N2_2 & $1.696(2)$ \\
\hline P2 & N2_4 & $1.695(2)$ \\
\hline N2_1 & $\mathrm{C}^{-} 1$ & $1.431(8)$ \\
\hline N2_1 & C5_1 & $1.429(8)$ \\
\hline C1_1 & C2_1 & $1.526(5)$ \\
\hline C2_1 & C3_1 & $1.522(6)$ \\
\hline C3_1 & C4_1 & $1.524(5)$ \\
\hline C4-1 & C5_1 & $1.523(5)$ \\
\hline $\mathrm{N} 2 \_2$ & C1_2 & $1.444(6)$ \\
\hline $\mathrm{N} 2-2$ & C5 2 & $1.423(10)$ \\
\hline C1_2 & $\mathrm{C} 2-2$ & $1.526(5)$ \\
\hline C2_2 & C3_2 & $1.523(6)$ \\
\hline C3_2 & C4_2 & $1.524(6)$ \\
\hline C4_2 & C5_2 & $1.522(6)$ \\
\hline N2_3 & C1_3 & $1.430(8)$ \\
\hline N2_3 & C5_3 & $1.413(9)$ \\
\hline C1_3 & C2_3 & $1.529(7)$ \\
\hline $\mathrm{C} 2-3$ & $\mathrm{C} 3-3$ & $1.521(7)$ \\
\hline C3_3 & C4_3 & $1.523(7)$ \\
\hline C4_3 & C5_3 & $1.523(7)$ \\
\hline N2_4 & C1_4 & $1.441(8)$ \\
\hline N2_4 & C5_4 & $1.421(10)$ \\
\hline C1_4 & $\mathrm{C} 2 \_4$ & $1.528(6)$ \\
\hline C2_4 & C3_4 & $1.524(6)$ \\
\hline C3_4 & C4_4 & $1.523(6)$ \\
\hline C4_4 & C5_4 & $1.522(6)$ \\
\hline
\end{tabular}


Table S5: Bond Angles in ${ }^{\circ}$ for 2-Ce.

\begin{tabular}{|c|c|c|c|}
\hline Atom & Atom & Atom & Angle $l^{\circ}$ \\
\hline $\mathrm{N} 1^{1}$ & $\mathrm{Ce} 1$ & $\mathrm{~N} 1^{2}$ & $111.8(6)$ \\
\hline $\mathrm{N} 1^{1}$ & $\mathrm{Ce} 1$ & N1 & 111.8(6) \\
\hline $\mathrm{N} 1^{2}$ & Ce1 & N1 & $111.8(6)$ \\
\hline $\mathrm{N} 1^{1}$ & $\mathrm{Ce} 1$ & N5 & 107.0(6) \\
\hline N1 & Ce1 & N5 & $107.0(6)$ \\
\hline $\mathrm{N} 1^{2}$ & $\mathrm{Ce} 1$ & N5 & 107.0(6) \\
\hline P2 & N1 & Ce1 & $153.7(15)$ \\
\hline P1 & N5 & $\mathrm{Ce} 1$ & 180.0 \\
\hline N5 & P1 & N2_3 ${ }^{1}$ & $110.0(6)$ \\
\hline N5 & P1 & N2_- $3^{2}$ & 110.0(6) \\
\hline N5 & P1 & N2_3 & $110.0(6)$ \\
\hline N2 3 & P1 & $\mathrm{N} 2^{-} 3^{1}$ & $108.9(6)$ \\
\hline $\mathrm{N} 23^{2}$ & P1 & $\mathrm{N} 2 \_3$ & $108.9(6)$ \\
\hline N2_3 $3^{2}$ & P1 & N2_3 ${ }^{1}$ & $108.9(6)$ \\
\hline N1 & P2 & N2_1 & $119.4(13)$ \\
\hline N1 & P2 & N2_2 & $113.7(13)$ \\
\hline N1 & P2 & $\mathrm{N} 2 \_4$ & $107.9(12)$ \\
\hline N2_2 & P2 & N2_1 & $104.4(6)$ \\
\hline N2_4 & P2 & N2_1 & $105.0(6)$ \\
\hline $\mathrm{N} 2-4$ & $\mathrm{P} 2$ & $\mathrm{~N} 2-2$ & $105.2(6)$ \\
\hline C1_1 & N2_1 & P2 & $112.5(11)$ \\
\hline C5_1 & N2_1 & P2 & $111.9(11)$ \\
\hline C5_1 & N2_1 & C1_1 & $114.0(12)$ \\
\hline N2_1 & C1_1 & C2_1 & $111.0(10)$ \\
\hline C3_1 & C2_1 & C1_1 & $111.1(10)$ \\
\hline $\mathrm{C} 2-1$ & C3_1 & C4_1 & $113.1(12)$ \\
\hline C5_1 & C4_1 & C3_1 & $114.2(11)$ \\
\hline N2_1 & C5_1 & C4_1 & $109.8(11)$ \\
\hline C1_2 & N2_2 & $\mathrm{P} 2$ & $115.6(12)$ \\
\hline C5_2 & N2_2 & P2 & $123.3(14)$ \\
\hline C5_2 & N2_2 & C1_2 & $114.3(12)$ \\
\hline N2_2 & C1_2 & C2_2 & $110.4(10)$ \\
\hline C3_2 & $\mathrm{C} 2 \_2$ & C1_2 & $111.0(10)$ \\
\hline C2_2 & C3_2 & C4_2 & $113.1(12)$ \\
\hline$C 5-2$ & C4_2 & $\mathrm{C} 3-2$ & $114.2(12)$ \\
\hline N2_2 & C5_2 & C4_2 & $110.5(12)$ \\
\hline C1_3 & N2_3 & P1 & $120.5(6)$ \\
\hline C5_3 & N2_3 & P1 & $118.0(6)$ \\
\hline C5_3 & N2_3 & C1_3 & $115.9(12)$ \\
\hline N2_3 & C1_3 & C2_3 & $111.5(8)$ \\
\hline C3_3 & C2_3 & C1_3 & 111.2(9) \\
\hline C2_3 & C3_3 & C4_3 & $113.6(12)$ \\
\hline C3_3 & C4_3 & C5_3 & $113.4(9)$ \\
\hline N2_3 & C5_3 & C4_3 & $110.3(11)$ \\
\hline C1_4 & N2_4 & P2 & $121.2(13)$ \\
\hline C5_4 & N2_4 & P2 & $121.6(14)$ \\
\hline C5_4 & N2_4 & C1_4 & $114.0(12)$ \\
\hline N2_4 & C1_4 & C2_4 & $109.7(11)$ \\
\hline C3_4 & C2_4 & C1_4 & 110.1(8) \\
\hline C4_4 & C3_4 & C2_4 & $113.0(12)$ \\
\hline C5_4 & C4_4 & C3_4 & $114.1(12)$ \\
\hline N2_4 & C5_4 & C4_4 & $110.2(12)$ \\
\hline
\end{tabular}

${ }^{1}-y,+x-y,+z ;{ }^{2}+y-x,-x,+z$ 
Table S6: Bond Lengths in $\AA$ for 3-Ce-(DME)2.

\begin{tabular}{|c|c|c|c|c|c|}
\hline Atom & Atom & Length/Å & Atom & Atom & Length/Å \\
\hline $\mathrm{Ce} 1$ & $\mathrm{~N} 1^{1}$ & $2.306(6)$ & $\overline{\mathrm{C} 2 \_8}$ & C3_8 & $1.531(7)$ \\
\hline Ce1 & N1 & $2.306(6)$ & C3_8 & C4_8 & $1.513(7)$ \\
\hline $\mathrm{Ce} 1$ & $\mathrm{~N} 2^{1}$ & $2.359(6)$ & C4_8 & C5_8 & $1.502(7)$ \\
\hline $\mathrm{Ce} 1$ & N2 & $2.359(6)$ & N5_9 & C5_9 & $1.469(5)$ \\
\hline N1 & $\mathrm{P} 1$ & $1.528(7)$ & N5_9 & C1_9 & $1.461(11)$ \\
\hline N2 & P2 & $1.529(6)$ & C5_9 & C4_9 & $1.544(13)$ \\
\hline $\mathrm{P} 1$ & N5 1 & $1.708(5)$ & C4_9 & C3_9 & $1.441(19)$ \\
\hline $\mathrm{P} 1$ & N5_2 & $1.713(7)$ & C3_9 & C2_9 & $1.526(18)$ \\
\hline P1 & N5_3 & $1.703(5)$ & C1_9 & C2_9 & $1.528(17)$ \\
\hline P1 & N5_4 & $1.620(12)$ & 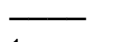 & & \\
\hline P2 & N5_5 & $1.680(5)$ & \multicolumn{3}{|c|}{$11-y+x, 2-y, 2 / 3-z$} \\
\hline P2 & N5_8 & $1.678(5)$ & & & \\
\hline P2 & N5_9 & $1.694(6)$ & & & \\
\hline 01_6 & C6_6 & $1.431(5)$ & & & \\
\hline $01-6$ & $\mathrm{C} 7{ }^{-6}$ & $1.436(5)$ & & & \\
\hline O2_6 & C8_6 & $1.432(5)$ & & & \\
\hline O2_6 & C9_6 & $1.435(5)$ & & & \\
\hline C7_6 & C8_6 & 1.481(15) & & & \\
\hline O1_7 & C6 67 & $1.431(5)$ & & & \\
\hline 01_7 & C7_7 & $1.436(5)$ & & & \\
\hline O2_7 & C8_7 & $1.432(5)$ & & & \\
\hline $\mathrm{O} 27$ & $\mathrm{C}^{-} 7$ & $1.435(5)$ & & & \\
\hline $\mathrm{C} 77$ & C8 7 & $1.481(15)$ & & & \\
\hline N5_1 & C1_1 & $1.469(5)$ & & & \\
\hline N5_1 & C5_1 & $1.479(5)$ & & & \\
\hline C1_1 & C2_1 & $1.502(6)$ & & & \\
\hline $\mathrm{C} 2 \_1$ & C3_1 & $1.529(7)$ & & & \\
\hline C3-1 & C4_-1 & $1.514(7)$ & & & \\
\hline C4_1 & C5_1 & $1.503(7)$ & & & \\
\hline N5_2 & $\mathrm{C} 1{ }^{-2} 2$ & $1.469(5)$ & & & \\
\hline N5_2 & C5_2 & $1.479(6)$ & & & \\
\hline C1_2 & C2_2 & $1.501(7)$ & & & \\
\hline C2_2 & C3_2 & $1.530(7)$ & & & \\
\hline $\mathrm{C} 3-2$ & $\mathrm{C} 4 \_-2$ & $1.514(7)$ & & & \\
\hline C4_2 & C5_2 & $1.503(7)$ & & & \\
\hline N5_3 & C1_3 & $1.468(5)$ & & & \\
\hline N5_3 & C5_3 & 1.477(5) & & & \\
\hline $\mathrm{C}^{-}{ }^{-3} 3$ & $\mathrm{C} 2{ }^{-} 3$ & $1.502(7)$ & & & \\
\hline C2_3 & C3_3 & $1.530(7)$ & & & \\
\hline C3_3 & C4_3 & $1.514(7)$ & & & \\
\hline C4_3 & C5_3 & $1.501(6)$ & & & \\
\hline N5_4 & C1_4 & $1.469(5)$ & & & \\
\hline N5_4 & C5_4 & $1.479(6)$ & & & \\
\hline C1_4 & C2_4 & $1.501(7)$ & & & \\
\hline $\mathrm{C} 2-4$ & $\mathrm{C}^{3}-4$ & $1.530(7)$ & & & \\
\hline C3_4 & C4_4 & $1.513(7)$ & & & \\
\hline C4_4 & C5_4 & $1.502(7)$ & & & \\
\hline N5_5 & C1_5 & $1.470(5)$ & & & \\
\hline N5_5 & C5_5 & $1.480(6)$ & & & \\
\hline C1_5 & C2_5 & $1.503(7)$ & & & \\
\hline C2_5 & C3_5 & $1.529(7)$ & & & \\
\hline C3_5 & C4_5 & $1.512(7)$ & & & \\
\hline C4_5 & C5 55 & $1.502(7)$ & & & \\
\hline N5_8 & C1_8 & $1.470(5)$ & & & \\
\hline N5_8 & C5_8 & $1.478(6)$ & & & \\
\hline C1_8 & C2_8 & $1.501(7)$ & & & \\
\hline
\end{tabular}


Table S7: Bond Angles in ${ }^{\circ}$ for $3-\mathrm{Ce}-\mathrm{K}(\mathrm{DME})_{2}$.

\begin{tabular}{|c|c|c|c|c|c|c|c|}
\hline Atom & Atom & Atom & Angle $^{\circ}$ & Atom & Atom & Atom & Angle $^{\circ}$ \\
\hline$\overline{\mathrm{N} 1{ }^{1}}$ & $\mathrm{Ce} 1$ & N1 & $109.3(3)$ & $\overline{\mathrm{C} 1 \_4}$ & N5_4 & C5_4 & $110.2(4)$ \\
\hline N1 & $\mathrm{Ce} 1$ & N2 & 110.3(3) & $\mathrm{C}^{-} 4$ & $\mathrm{~N} 5^{-} 4$ & $\mathrm{P} 1^{-}$ & 127.4(10) \\
\hline $\mathrm{N} 1^{1}$ & $\mathrm{Ce} 1$ & $\mathrm{~N} 2^{1}$ & $110.3(3)$ & N5 4 & $\mathrm{C} 1-4$ & C2 4 & $110.3(5)$ \\
\hline $\mathrm{N} 1$ & $\mathrm{Ce} 1$ & $\mathrm{~N} 2^{1}$ & $115.0(2)$ & C1_4 & C2_4 & C3_4 & $110.9(5)$ \\
\hline $\mathrm{N} 1{ }^{1}$ & $\mathrm{Ce} 1$ & N2 & $115.0(2)$ & C4_4 & C3_4 & $\mathrm{C} 2{ }^{-} 4$ & $111.4(5)$ \\
\hline $\mathrm{N} 2^{1}$ & $\mathrm{Ce} 1$ & N2 & $96.5(3)$ & $\mathrm{C}^{5} 4$ & $\mathrm{C} 4-4$ & $\mathrm{C} 34$ & $110.9(5)$ \\
\hline P1 & $\mathrm{N} 1$ & $\mathrm{Ce} 1$ & $173.1(4)$ & N5_4 & C5_4 & C4_4 & $111.0(5)$ \\
\hline P2 & N2 & $\mathrm{Ce} 1$ & $141.4(4)$ & C1_5 & N5_5 & $\mathrm{P} 2$ & $125.1(4)$ \\
\hline N1 & P1 & N5_1 & $120.5(4)$ & C1_5 & N5_5 & C5_5 & $109.9(4)$ \\
\hline N1 & P1 & $\mathrm{N} 5{ }^{-} 2$ & 114.1(4) & $\mathrm{C} 55$ & N5 5 & $\mathrm{P} 2$ & $115.4(4)$ \\
\hline N1 & P1 & $\mathrm{N} 53$ & $113.1(3)$ & N5_5 & C1_5 & C2_5 & $109.7(4)$ \\
\hline N1 & P1 & N5_4 & $114.1(7)$ & C1_5 & C2_5 & C3_5 & $110.8(5)$ \\
\hline N5_1 & P1 & N5_2 & $100.4(4)$ & C4_5 & C3_5 & $\mathrm{C} 2-5$ & $111.7(5)$ \\
\hline N5 3 & P1 & $\mathrm{N} 5^{-} 1$ & $98.8(3)$ & C5_5 & $\mathrm{C} 4-5$ & C3 5 & $111.2(5)$ \\
\hline N5_3 & P1 & N5_2 & $108.0(5)$ & N5_5 & C5_5 & C4_5 & $111.0(4)$ \\
\hline N5 4 & $\mathrm{P} 1$ & N5 1 & $98.8(7)$ & C1_8 & N5_8 & $\mathrm{P} 2$ & $122.9(4)$ \\
\hline N5_4 & P1 & N5_3 & 109.6(8) & C1_8 & N5_8 & C5_8 & $110.3(4)$ \\
\hline N2 & P2 & N5 5 & $113.6(3)$ & C5 58 & N5_8 & $\mathrm{P} 2$ & $123.8(4)$ \\
\hline N2 & P2 & N5 8 & $114.8(4)$ & N5_8 & C1_8 & C2_8 & $110.2(4)$ \\
\hline N2 & P2 & N5_9 & $119.8(4)$ & C1_8 & C2_8 & C3_8 & $110.6(5)$ \\
\hline N5 5 & P2 & N5 9 & $99.7(3)$ & $\mathrm{C}^{-} 8$ & $\mathrm{C} 3{ }^{-} 8$ & $\mathrm{C} 28$ & $111.1(5)$ \\
\hline N5 8 & P2 & N5 5 & $105.8(3)$ & C5 58 & $\mathrm{C} 4-8$ & C3 3 & $110.8(5)$ \\
\hline N5 8 & P2 & N5 9 & 101.1(3) & N5_8 & C5_8 & C4_8 & $111.2(4)$ \\
\hline C6_6 & 01_6 & C7_6 & $111.7(11)$ & C5_9 & N5_9 & $\mathrm{P} 2$ & $115.0(5)$ \\
\hline C8_6 & $02 \_6$ & C9_6 & $113.0(10)$ & C1_9 & N5_9 & P2 & $115.1(6)$ \\
\hline $01^{-} 6$ & $\mathrm{C}^{-} 6$ & $\mathrm{C} 8{ }^{-} 6$ & $110.0(8)$ & C1_9 & N5 9 & C5 9 & $108.3(7)$ \\
\hline O2_6 & C8_6 & C7_6 & 111.1(9) & N5_9 & C5_9 & C4_9 & 107.2(7) \\
\hline C6 7 & $01^{-} 7$ & $\mathrm{C} 7^{-} 7$ & $111.6(11)$ & $\mathrm{C} 3{ }^{-} 9$ & $\mathrm{C} 4{ }^{-} 9$ & $\mathrm{C} 5{ }^{-} 9$ & 111.2(11) \\
\hline $\mathrm{C}^{-} 7$ & $\mathrm{O}^{-7} 7$ & $\mathrm{Cg}^{-} 7$ & $113.0(9)$ & $\mathrm{C}^{4}-9$ & $\mathrm{C} 3-9$ & $\mathrm{C} 2-9$ & $111.2(10)$ \\
\hline $\mathrm{O}^{-} 7$ & $\mathrm{C} 7^{-7}$ & $\mathrm{C} 87$ & $110.0(8)$ & N5_9 & C1_9 & $\mathrm{C} 2 \_9$ & $107.2(9)$ \\
\hline $\mathrm{O} 2 \_7$ & C8_7 & $\mathrm{C} 7 \_7$ & $111.0(8)$ & C3_9 & C2_9 & C1_9 & $109.8(11)$ \\
\hline C1_1 & N5_1 & $\mathrm{P} 1$ & $115.3(4)$ & - & & & \\
\hline C1_1 & N5_1 & C5_1 & $110.2(4)$ & & 2/3-z & & \\
\hline
\end{tabular}

$\begin{array}{llll}\text { C5_1 } & \text { N5_1 } & \text { P1 } & 115.4(4) \\ \text { N5_1 } & \text { C1_1 } & \text { C2_1 } & 110.3(4) \\ \text { C1_1 } & \text { C2_1 } & \text { C3_1 } & 110.9(5) \\ \text { C4_1 } & \text { C3_1 } & \text { C2_1 } & 111.4(5) \\ \text { C5_1 } & \text { C4_1 } & \text { C3_1 } & 110.8(5) \\ \text { N5_1 } & \text { C5_1 } & \text { C4_1 } & 111.0(4) \\ \text { C1_2 } & \text { N5_2 } & \text { P1 } & 118.0(6) \\ \text { C1_2 } & \text { N5_2 } & \text { C5_2 } & 110.3(4) \\ \text { C5_2 } & \text { N5_2 } & \text { P1 } & 123.6(6) \\ \text { N5_2 } & \text { C1_2 } & \text { C2_2 } & 110.4(5) \\ \text { C1_2 } & \text { C2_2 } & \text { C3_2 } & 110.9(5) \\ \text { C4_2 } & \text { C3_2 } & \text { C2_2 } & 111.3(5) \\ \text { C5_2 } & \text { C4_2 } & \text { C3_2 } & 110.7(5) \\ \text { N5_2 } & \text { C5_2 } & \text { C4_2 } & 110.9(5) \\ \text { C1_3 } & \text { N5_3 } & \text { P1 } & 120.4(4) \\ \text { C1_3 } & \text { N5_3 } & \text { C5_3 } & 110.5(4) \\ \text { C5_3 } & \text { N5_3 } & \text { P1 } & 114.0(4) \\ \text { N5_3 } & \text { C1_3 } & \text { C2_3 } & 110.2(4) \\ \text { C1_3 } & \text { C2_3 } & \text { C3_3 } & 110.6(5) \\ \text { C4_3 } & \text { C3_3 } & \text { C2_3 } & 111.1(4) \\ \text { C5_3 } & \text { C4_3 } & \text { C3_3 } & 110.9(5) \\ \text { N5_3 } & \text { C5_3 } & \text { C4_3 } & 111.4(4) \\ \text { C1_4 } & \text { N5_4 } & \text { P1 } & 120.6(10)\end{array}$


Table S8: Bond Lengths in $\AA$ for $3-\mathrm{Ce}-\left(\mathrm{Et}_{2} \mathrm{O}\right)$.

\begin{tabular}{|c|c|c|}
\hline Atom & Atom & Length/A \\
\hline K1 & N5_3 & $2.717(9)$ \\
\hline K1 & N1) 3 & $2.660(11)$ \\
\hline K1 & 01_e & $2.760(6)$ \\
\hline K1 & 01_9 & $2.762(6)$ \\
\hline K2 & N5_1 & $2.728(9)$ \\
\hline K2 & $\mathrm{N} 1 \overline{3} 1$ & $2.696(10)$ \\
\hline K2 & $01 \overline{4}$ & $2.761(6)$ \\
\hline K2 & 01_5 & $2.761(6)$ \\
\hline K3 & N5_2 & $2.709(9)$ \\
\hline K3 & N13̄_2 & $2.712(11)$ \\
\hline K3 & 01_̄̄ & $2.761(6)$ \\
\hline K3 & 01 0 & $2.763(6)$ \\
\hline Ce1_2 & N1_2 & $2.299(7)$ \\
\hline Ce1_2 & N5_2 & $2.372(7)$ \\
\hline Ce1_2 & N9_2 & $2.312(8)$ \\
\hline Ce1_2 & N13_2 & $2.360(8)$ \\
\hline P1_̄ & N1_产 & $1.523(8)$ \\
\hline P1_2 & $\mathrm{N} 2{ }^{-} 2$ & $1.708(9)$ \\
\hline P1_2 & N3_2 & $1.688(8)$ \\
\hline P1_2 & N4_2 & $1.701(8)$ \\
\hline $\mathrm{P} 2{ }^{-} 2$ & $\mathrm{~N} 5{ }^{-} 2$ & $1.544(7)$ \\
\hline $\mathrm{P} 2{ }^{-} 2$ & N6_2 & $1.707(7)$ \\
\hline$P 2$ & N7_2 & $1.692(8)$ \\
\hline $\mathrm{P} 2 \_2$ & N8_2 & $1.680(7)$ \\
\hline P3_2 & N9_2 & $1.522(8)$ \\
\hline P3_2 & N10_2 & $1.667(9)$ \\
\hline P3_2 & N11_2 & $1.671(9)$ \\
\hline P3_2 & N12_2 & $1.725(9)$ \\
\hline $\mathrm{P}^{-}{ }^{-} 2$ & N13 2 & $1.518(8)$ \\
\hline P4_2 & N14_2 & $1.674(10)$ \\
\hline P4_2 & N15_2 & $1.678(10)$ \\
\hline P4_2 & N16_2 & $1.682(10)$ \\
\hline N2_2 & C1_2 & $1.447(13)$ \\
\hline N2_2 & C5_2 & $1.489(14)$ \\
\hline N3_2 & C6_2 & $1.459(11)$ \\
\hline N3_2 & C10_2 & $1.461(11)$ \\
\hline $\mathrm{N} 4-2$ & $\mathrm{C} 11^{-} 2$ & $1.478(12)$ \\
\hline N4_2 & C15_2 & $1.478(12)$ \\
\hline N6_2 & C16_2 & $1.484(10)$ \\
\hline N6_2 & C20_2 & $1.464(10)$ \\
\hline N7_2 & C21_2 & $1.452(11)$ \\
\hline N7_2 & C25_2 & $1.459(11)$ \\
\hline N8_2 & C26_2 & $1.469(10)$ \\
\hline N8_2 & C30_2 & $1.482(11)$ \\
\hline$N 1 \overline{0} 2$ & C31_2 & $1.445(13)$ \\
\hline N10_2 & C35_2 & $1.457(12)$ \\
\hline N11_2 & C36_2 & $1.409(15)$ \\
\hline N11_2 & C40_2 & $1.391(16)$ \\
\hline N12_2 & C41_2 & $1.444(14)$ \\
\hline N12_2 & C45_2 & $1.480(14)$ \\
\hline N14_2 & C46_2 & $1.442(15)$ \\
\hline N14_2 & C50_2 & $1.427(14)$ \\
\hline N15_2 & C51_2 & $1.500(16)$ \\
\hline
\end{tabular}

\begin{tabular}{lll}
\hline Atom & Atom & Length/A \\
\hline N15_2 & C55_2 & $1.393(16)$ \\
N16_2 & C56_2 & $1.540(16)$ \\
N16_2 & C60_2 & $1.440(14)$ \\
C1_2 & C2_2 & $1.510(9)$ \\
C2_2 & C3_2 & $1.520(9)$ \\
C3_2 & C4_2 & $1.528(9)$ \\
C4_2 & C5_2 & $1.519(9)$ \\
C6_2 & C7_2 & $1.519(8)$ \\
C7_2 & C8_2 & $1.529(8)$ \\
C8_2 & C9_2 & $1.520(8)$ \\
C9_2 & C10_2 & $1.512(8)$ \\
C11_2 & C12_2 & $1.525(8)$ \\
C12_2 & C13_2 & $1.512(9)$ \\
C13_2 & C14_2 & $1.532(8)$ \\
C14_2 & C15_2 & $1.525(8)$ \\
C16_2 & C17_2 & $1.531(8)$ \\
C17_2 & C18_2 & $1.531(8)$ \\
C18_2 & C19_2 & $1.521(8)$ \\
C19_2 & C20_2 & $1.521(8)$ \\
C21_2 & C22_2 & $1.526(8)$ \\
C22_2 & C23_2 & $1.514(8)$ \\
C23_2 & C24_2 & $1.525(8)$ \\
C24_2 & C25_2 & $1.533(8)$ \\
C26_2 & C27_2 & $1.525(8)$ \\
C27_2 & C28_2 & $1.524(9)$ \\
C28_2 & C29_2 & $1.521(8)$ \\
C29_2 & C30_2 & $1.511(8)$ \\
C31_2 & C32_2 & $1.520(9)$ \\
C32_2 & C33_2 & $1.527(9)$ \\
C33_2 & C34_2 & $1.534(9)$ \\
C34_2 & C35_2 & $1.516(9)$ \\
C36_2 & C37_2 & $1.545(9)$ \\
C37_2 & C38_2 & $1.517(9)$ \\
C38_2 & C39_2 & $1.527(9)$ \\
C39_2 & C40_2 & $1.539(9)$ \\
C41_2 & C42_2 & $1.531(9)$ \\
C42_2 & C43_2 & $1.507(9)$ \\
C43_2 & C44_2 & $1.523(9)$ \\
C44_2 & C45_2 & $1.531(9)$ \\
C46_2 & C47_2 & $1.527(8)$ \\
C47_2 & C48_2 & $1.512(8)$ \\
C48_2 & C49_2 & $1.499(9)$ \\
C49_2 & C50_2 & $1.505(9)$ \\
C51_2 & C52_2 & $1.534(9)$ \\
C52_2 & C53_2 & $1.528(9)$ \\
C53_2 & C54_2 & $1.501(9)$ \\
C54_2 & C55_2 & $1.500(9)$ \\
C56_2 & C57_2 & $1.547(9)$ \\
C57_2 & C58_2 & $1.5219)$ \\
C58_2 & C59_2 & $1.504(9)$ \\
C59_2 & C60_2 & $1.507(9)$ \\
Ce113 & N1_3 & $2.295(8)$ \\
Ce1_3 & N5_3 & $2.366(7)$ \\
Ce1_3 & N9_3 & $2.308(8)$ \\
& &
\end{tabular}




\begin{tabular}{|c|c|c|}
\hline Atom & Atom & Length/Å \\
\hline Ce1_3 & N13_3 & $2.374(8)$ \\
\hline P1_ $\overline{3}$ & N1_ $\overline{3}$ & $1.518(8)$ \\
\hline P1_3 & N2_3 & $1.709(9)$ \\
\hline P1_3 & N3_3 & $1.691(8)$ \\
\hline P1_3 & N4_3 & $1.695(8)$ \\
\hline P2_3 & N5_3 & $1.547(7)$ \\
\hline P2_3 & N6_3 & $1.711(8)$ \\
\hline P2_3 & N7_3 & 1.684(8) \\
\hline P2_3 & N8_3 & $1.671(8)$ \\
\hline P3_3 & N9_3 & $1.526(8)$ \\
\hline P3_3 & N10_3 & 1.667(9) \\
\hline P3_3 & N11_3 & $1.683(9)$ \\
\hline P3_3 & N12_3 & $1.718(9)$ \\
\hline P4_3 & N13_3 & $1.506(8)$ \\
\hline P4_3 & N14_3 & 1.694(10) \\
\hline P4_3 & N15_3 & 1.666(10) \\
\hline P4_3 & N16_3 & 1.687(10) \\
\hline N2_3 & C1_3 & $1.456(13)$ \\
\hline N2_3 & C5_3 & $1.497(14)$ \\
\hline N3_3 & C6_3 & $1.457(12)$ \\
\hline N3_3 & C10_3 & $1.462(12)$ \\
\hline N4_3 & C11_3 & $1.474(12)$ \\
\hline N4_3 & C15_3 & 1.492(13) \\
\hline N6_3 & C16_3 & $1.485(11)$ \\
\hline N6_3 & C20_3 & $1.468(11)$ \\
\hline N7_3 & C21_3 & $1.453(11)$ \\
\hline N7_3 & C25_3 & $1.460(11)$ \\
\hline N8_3 & C26_3 & $1.461(11)$ \\
\hline N8_3 & C30_3 & $1.479(11)$ \\
\hline N10_3 & C31_3 & $1.440(13)$ \\
\hline N10_3 & C35_3 & $1.450(13)$ \\
\hline N11_3 & C36_3 & $1.421(15)$ \\
\hline N11_3 & C40_3 & $1.391(15)$ \\
\hline N12_3 & C41_3 & $1.439(14)$ \\
\hline N12_3 & C45_3 & $1.476(13)$ \\
\hline N14_3 & C46_3 & $1.439(15)$ \\
\hline N14_3 & C50_3 & $1.428(15)$ \\
\hline N15_3 & C51_3 & $1.500(16)$ \\
\hline N15_3 & C55_3 & $1.383(16)$ \\
\hline N16_3 & C56_3 & $1.538(16)$ \\
\hline N16_3 & C60_3 & $1.445(14)$ \\
\hline C1_ $\overline{3}$ & C2_ 3 & $1.515(6)$ \\
\hline C2_3 & C3_3 & $1.521(6)$ \\
\hline C3_3 & C4_3 & $1.525(6)$ \\
\hline C4_3 & C5_3 & $1.517(6)$ \\
\hline C6_3 & C7_3 & $1.521(5)$ \\
\hline C7_3 & C8_3 & $1.526(5)$ \\
\hline C8_3 & C9_3 & $1.524(5)$ \\
\hline C9_3 & C10_3 & $1.520(5)$ \\
\hline C11_3 & C12_3 & $1.526(5)$ \\
\hline C12_3 & C13_3 & $1.516(6)$ \\
\hline C13_3 & C14_3 & $1.529(6)$ \\
\hline C14_3 & C15_3 & $1.524(5)$ \\
\hline C16_3 & C17_3 & $1.526(5)$ \\
\hline C17_3 & C18_3 & $1.528(5)$ \\
\hline C18_3 & C19_3 & $1.524(5)$ \\
\hline C19_3 & C20_3 & $1.525(5)$ \\
\hline C21_3 & C22_3 & $1.525(5)$ \\
\hline C22_3 & C23_3 & $1.518(5)$ \\
\hline
\end{tabular}

\begin{tabular}{|c|c|c|}
\hline Atom & Atom & Length/Å \\
\hline$\overline{C 23 \_3}$ & C24_3 & $1.524(5)$ \\
\hline C24_3 & C25_3 & $1.531(5)$ \\
\hline C26_3 & C27_3 & $1.528(5)$ \\
\hline C27_3 & C28_3 & $1.527(6)$ \\
\hline C28_3 & C29_3 & $1.524(5)$ \\
\hline C29_3 & C30_3 & $1.518(5)$ \\
\hline C31_3 & C32_3 & $1.519(6)$ \\
\hline C32_3 & C33_3 & $1.525(6)$ \\
\hline C33_3 & C34_3 & $1.529(6)$ \\
\hline C34_3 & C35_3 & $1.517(6)$ \\
\hline C36_3 & C37_3 & $1.532(6)$ \\
\hline C37_3 & C38_3 & $1.519(6)$ \\
\hline C38_3 & C39_3 & $1.524(6)$ \\
\hline C39_3 & C40_3 & $1.530(6)$ \\
\hline C41_3 & C42_3 & $1.526(6)$ \\
\hline C42_3 & C43_3 & $1.515(6)$ \\
\hline C43_3 & C44_3 & $1.523(6)$ \\
\hline C44_3 & C45_3 & $1.526(6)$ \\
\hline C46_3 & C47_3 & $1.520(6)$ \\
\hline C47_3 & C48_3 & $1.515(5)$ \\
\hline C48_3 & C49_3 & $1.515(6)$ \\
\hline C49_3 & C50_3 & $1.517(6)$ \\
\hline C51_3 & C52_3 & $1.531(6)$ \\
\hline C52_3 & C53_3 & $1.527(6)$ \\
\hline C53_3 & C54_3 & $1.514(6)$ \\
\hline C54_3 & C55_3 & $1.515(6)$ \\
\hline C56_3 & C57_3 & $1.529(6)$ \\
\hline C57_3 & C58_3 & $1.520(6)$ \\
\hline C58_3 & C59_3 & $1.517(6)$ \\
\hline C59_3 & C60_3 & $1.518(6)$ \\
\hline 01_ 7 & C2 $2 \overline{7}$ & $1.412(3)$ \\
\hline O1_7 & C3_7 & $1.419(3)$ \\
\hline C1_7 & $\mathrm{C} 2-7$ & $1.518(3)$ \\
\hline C3_7 & C4_7 & $1.520(3)$ \\
\hline 01_6 & C2_6 & $1.414(3)$ \\
\hline 01_6 & C3_6 & $1.421(3)$ \\
\hline C1_6 & C2_6 & 1.518(3) \\
\hline C3_6 & C4_6 & $1.519(3)$ \\
\hline 01_8 & C2_8 & $1.411(3)$ \\
\hline 01_8 & C3_8 & $1.419(3)$ \\
\hline C1_8 & C2_8 & $1.519(3)$ \\
\hline C3_8 & C4_8 & $1.520(3)$ \\
\hline Ce'1_1 & N1_1 & $2.301(7)$ \\
\hline $\mathrm{Ce}^{-1} 1$ & N5_1 & $2.369(7)$ \\
\hline $\mathrm{Ce}^{-1} 1$ & N9_1 & $2.311(8)$ \\
\hline Ce1_1 & N13_1 & $2.365(8)$ \\
\hline P1_ 1 & N1_ 1 & $1.516(8)$ \\
\hline P1_1 & N2_1 & $1.703(9)$ \\
\hline P1-1 & N3_1 & 1.696(8) \\
\hline P1_1 & N4_1 & 1.697(8) \\
\hline $\mathrm{P} 2{ }^{-} 1$ & N5_1 & $1.544(7)$ \\
\hline P2_1 & N6_1 & $1.707(7)$ \\
\hline P2_1 & N7_-1 & 1.684(8) \\
\hline P2_1 & N8_1 & $1.682(7)$ \\
\hline P3_1 & N9_1 & $1.528(8)$ \\
\hline P3-1 & N10_1 & $1.663(9)$ \\
\hline P3-1 & N11_1 & 1.683(9) \\
\hline P3-1 & N12_1 & $1.720(9)$ \\
\hline P4_1 & N13_1 & $1.525(8)$ \\
\hline
\end{tabular}




\begin{tabular}{|c|c|c|}
\hline Atom & Atom & Length/Å \\
\hline$\overline{\mathrm{P} 4 \_1}$ & N14_1 & $1.678(10)$ \\
\hline P4_1 & N15_1 & $1.664(10)$ \\
\hline P4_1 & N16_1 & $1.679(10)$ \\
\hline $\mathrm{N} 2-1$ & C1 $\overline{1}$ & $1.440(13)$ \\
\hline N2_1 & C5_1 & $1.490(14)$ \\
\hline N3_1 & C6_-1 & $1.460(11)$ \\
\hline N3 ${ }^{-} 1$ & $\mathrm{C} 1 \overline{0} 1$ & $1.462(11)$ \\
\hline $\mathrm{N} 4-1$ & $\mathrm{C} 11^{-} 1$ & $1.482(12)$ \\
\hline N4_1 & C15_1 & $1.487(12)$ \\
\hline N6_1 & C16_1 & $1.490(10)$ \\
\hline N6_1 & $\mathrm{C} 2 \mathrm{C}_{-}^{-1}$ & $1.463(10)$ \\
\hline $\mathrm{N} 7-1$ & $\mathrm{C} 21^{-} 1$ & $1.452(11)$ \\
\hline N7_1 & C25_1 & $1.460(11)$ \\
\hline N8_-1 & $\mathrm{C} 266_{-1}^{-1}$ & 1.464(10) \\
\hline N8-1 & $\mathrm{C} 30^{-} 1$ & $1.479(11)$ \\
\hline N10_ 1 & C31_1 & $1.442(13)$ \\
\hline N10_1 & C35_1 & $1.452(13)$ \\
\hline N11_1 & C36_1 & $1.418(15)$ \\
\hline N11_1 & C40_1 & $1.390(16)$ \\
\hline N12_1 & C41_1 & $1.434(14)$ \\
\hline N12_1 & C45_1 & $1.482(13)$ \\
\hline N14_1 & $\mathrm{C} 46-1$ & $1.432(15)$ \\
\hline $\mathrm{N} 14^{-} 1$ & $\mathrm{C} 50^{-1}$ & $1.420(15)$ \\
\hline N15_1 & C51_1 & $1.494(16)$ \\
\hline N15_1 & C55_1 & $1.401(15)$ \\
\hline N16_1 & C56_1 & $1.517(16)$ \\
\hline N16_1 & $\mathrm{C} 60^{-} 1$ & $1.445(14)$ \\
\hline C1_1 & C2 $2 \overline{1}$ & $1.514(9)$ \\
\hline $\mathrm{C} 2-1$ & $\mathrm{C} 3-1$ & $1.517(9)$ \\
\hline $\mathrm{C} 3{ }^{-1}$ & $\mathrm{C}^{-} 1$ & $1.528(9)$ \\
\hline C4_1 & $\mathrm{C} 5$ & $1.525(8)$ \\
\hline C6_1 & C7_1 & $1.514(8)$ \\
\hline C7_1 & C8_1 & $1.524(8)$ \\
\hline C8_1 & C9_1 & $1.522(8)$ \\
\hline $\mathrm{C}^{-} \mathrm{1}$ & $C 1 \overline{0} \_1$ & $1.515(8)$ \\
\hline C1̄1_1 & C12_1 & $1.524(8)$ \\
\hline C12_1 & C13_1 & $1.515(9)$ \\
\hline $\mathrm{C} 13^{-1}$ & $\mathrm{C} 14^{-} 1$ & $1.534(8)$ \\
\hline C14_1 & C15_1 & $1.528(8)$ \\
\hline C16_1 & C17_1 & $1.527(8)$ \\
\hline C17_1 & C18_1 & $1.529(8)$ \\
\hline C18_1 & C19_1 & $1.519(8)$ \\
\hline C19_1 & $\mathrm{C} 2 \mathrm{C}_{-}^{-1}$ & $1.522(8)$ \\
\hline C21_1 & C22_1 & 1.528(8) \\
\hline $\mathrm{C} 22^{-1}$ & $\mathrm{C} 23^{-} 1$ & $1.510(8)$ \\
\hline $\mathrm{C} 23^{-1}$ & $\mathrm{C} 24^{-1} 1$ & $1.524(8)$ \\
\hline C24_1 & C25_1 & $1.535(8)$ \\
\hline C26_1 & C27_1 & $1.526(8)$ \\
\hline C27_1 & C28_1 & $1.529(8)$ \\
\hline $\mathrm{C} 28-1$ & $\mathrm{C} 29-1$ & $1.521(8)$ \\
\hline $\mathrm{C} 29^{-1}$ & $\mathrm{C} 30^{-} 1$ & $1.511(8)$ \\
\hline
\end{tabular}

\begin{tabular}{lll}
\hline Atom & Atom & Length/A \\
\hline C31_1 & C32_1 & $1.515(9)$ \\
C32_1 & C33_1 & $1.530(9)$ \\
C33_1 & C34_1 & $1.537(9)$ \\
C34_1 & C35_1 & $1.509(9)$ \\
C36_1 & C37_1 & $1.542(9)$ \\
C37_1 & C38_1 & $1.515(9)$ \\
C38_1 & C39_1 & $1.524(9)$ \\
C39_1 & C40_1 & $1.543(9)$ \\
C41_1 & C42_1 & $1.534(9)$ \\
C42_1 & C43_1 & $1.507(9)$ \\
C43_1 & C44_1 & $1.527(9)$ \\
C44_1 & C45_1 & $1.527(9)$ \\
C46_1 & C47-1 & $1.520(8)$ \\
C47_1 & C48_1 & $1.512(8)$ \\
C48_1 & C49_1 & $1.508(9)$ \\
C49_1 & C50_1 & $1.506(9)$ \\
C51_1 & C52_1 & $1.541(9)$ \\
C52_1 & C53_1 & $1.525(9)$ \\
C53_1 & C54_1 & $1.511(9)$ \\
C54_1 & C55_1 & $1.513(9)$ \\
C56_1 & C57_1 & $1.539(9)$ \\
C57_1 & C58_1 & $1.517(9)$ \\
C58_1 & C59_1 & $1.512(9)$ \\
C59_1 & C60_1 & $1.518(9)$ \\
O1_4 & C2_4 & $1.411(3)$ \\
O1_4 & C3_4 & $1.419(3)$ \\
C1_4 & C2_4 & $1.518(3)$ \\
C3_4 & C4_4 & $1.520(3)$ \\
O1_5 & C2_5 & $1.411(3)$ \\
O1_5 & C3_5 & $1.418(3)$ \\
C1_5 & C2_5 & $1.519(3)$ \\
C3_5 & C4_5 & $1.520(3)$ \\
O1_9 & C2_9 & $1.413(3)$ \\
O1_9 & C3_9 & $1.420(3)$ \\
C1_9 & C2_9 & $1.518(3)$ \\
C3_9 & C4_9 & $1.519(3)$ \\
& &
\end{tabular}


Table S9: Bond Angles in ${ }^{\circ}$ for $3-\mathrm{Ce}-\left(\mathrm{Et}_{2} \mathrm{O}\right)$.

\begin{tabular}{|c|c|c|c|c|c|c|c|}
\hline Atom & Atom & Atom & Angle $^{\circ}$ & Atom & Atom & Atom & Angle $^{\circ}$ \\
\hline N5 3 & $\mathrm{~K} 1$ & 018 & $108.9(4)$ & $\overline{C 1} 2$ & N2_2 & $\mathrm{P} 1$ 1_2 & $121.9(8)$ \\
\hline N5_3 & $\mathrm{K} 1$ & 01_9 & $90.0(4)$ & C1_2 & N2_2 & C5_2 & $113.5(10)$ \\
\hline N13 3 & $\mathrm{~K} 1$ & N5_3 & $78.3(2)$ & C5_2 & N2_2 & P1_2 & $115.4(7)$ \\
\hline N13_3 & $\mathrm{K} 1$ & 01_8 & $112.5(4)$ & C6_2 & N3_2 & $\mathrm{P} 1 \_2$ & $123.3(7)$ \\
\hline N13 3 & K1 & $01-9$ & $95.7(4)$ & C6_2 & N3_2 & C10_2 & $111.8(8)$ \\
\hline 01_e & K1 & 01_9 & $148.4(4)$ & C10_2 & N3_2 & P1_z & $115.8(7)$ \\
\hline N5 ${ }^{-1}$ & K2 & $01^{-4}$ & $105.0(4)$ & $\mathrm{C} 11^{-} 2$ & $\mathrm{~N} 4-2$ & $\mathrm{P}^{-}{ }^{-} 2$ & $114.7(7)$ \\
\hline N5 ${ }^{-1}$ & K2 & $\mathrm{O}^{-} 5$ & $109.6(3)$ & C15_2 & $\mathrm{N} 4 \_2$ & $\mathrm{P} 1{ }^{-} 2$ & $116.6(6)$ \\
\hline N1) $\_1$ & $\mathrm{~K} 2$ & N5_1 & $78.1(2)$ & C15_2 & N4_2 & C11_2 & $111.4(9)$ \\
\hline N13_1 & $\mathrm{K} 2$ & O1_4 & $115.2(4)$ & Ce1_2 & N5_2 & K3 & $94.3(2)$ \\
\hline N13_1 & $\mathrm{K} 2$ & 01_5 & $113.7(4)$ & P2_ 2 & N5_2 & K3 & $127.1(4)$ \\
\hline $01 \_\overline{5}$ & $\mathrm{~K} 2$ & O1_4 & $124.3(4)$ & $\mathrm{P} 2$-2 & N5_2 & Ce1_2 & $136.6(5)$ \\
\hline N5 2 & K3 & $\mathrm{N} 1 \overline{3} 2$ & $77.8(2)$ & C1̄﹎_2 & N6_2 & P2_ $\overline{2}$ & $115.2(6)$ \\
\hline N5 2 & K3 & $01 \overline{7}$ & $108.9(4)$ & C20_2 & N6_2 & P2_2 & $124.0(7)$ \\
\hline N5 2 & K3 & $01^{-} 6$ & $100.3(3)$ & $\mathrm{C} 2 \mathrm{O}^{-} 2$ & N6 2 & C1̄ 2 & $110.1(8)$ \\
\hline N13﹎2 & K3 & 01_7 & $110.5(5)$ & C21_2 & N7_2 & $\mathrm{P} 2 \overline{2}^{-}$ & $122.8(6)$ \\
\hline N13_2 & K3 & 01_6 & $114.5(3)$ & C21_2 & N7_2 & C25_2 & 113.1(8) \\
\hline 01 & K3 & 01_6 & $130.3(4)$ & C25_2 & N7_2 & $\mathrm{P} 2 \_\overline{2}$ & $120.8(6)$ \\
\hline N1_2 & Ce1_2 & N5_2 & $112.6(3)$ & C26_2 & N8_2 & P2_2 & 115.6(6) \\
\hline $\mathrm{N} 1^{-} 2$ & $\mathrm{Ce}^{-1}{ }^{-} 2$ & $\mathrm{~N}^{-} 2$ & $106.9(3)$ & C26_2 & N8_2 & C30_2 & 107.1(8) \\
\hline N1_2 & Ce1_2 & N1) & $114.1(3)$ & C30_2 & N8_2 & P2_ 2 & $115.4(6)$ \\
\hline N9_2 & Ce1_2 & N5_ 2 & $115.0(3)$ & P3_ 2 & N9_2 & $\mathrm{Ce} \overline{1} \_2$ & $165.0(7)$ \\
\hline $\mathrm{N}^{-} 2$ & $\mathrm{Ce}^{-}{ }^{-} 2$ & $\mathrm{~N} 1 \overline{3} 2$ & 116.1(3) & $\mathrm{C} 3 \overline{1} 2$ & $\mathrm{~N} 1 \overline{0} 2$ & P3 $\overline{2}$ & $118.3(7)$ \\
\hline $\mathrm{N} 1 \overline{3} \_2$ & Ce1_2 & N5_ 2 & $92.0(3)$ & C31_2 & N10_2 & C35_2 & $112.3(9)$ \\
\hline N1 $\overline{2}$ & $\mathrm{P} 1 \overline{2}^{2}$ & N2 2 & $113.9(5)$ & C35_2 & N10_2 & P3_ 2 & $122.5(7)$ \\
\hline N1_2 & P1_2 & N3_2 & $113.0(5)$ & C36_2 & N11_2 & P3_2 & $119.0(8)$ \\
\hline $\mathrm{N} 1^{-} 2$ & $\mathrm{P}^{-} 2$ & $\mathrm{~N} 4-2$ & $121.3(5)$ & $\mathrm{C} 40^{-} 2$ & N11_2 & $\mathrm{P} 3-2$ & $118.7(8)$ \\
\hline N3 $3-2$ & $\mathrm{P} 12$ & $\mathrm{~N} 2-2$ & $110.1(5)$ & C40_2 & N11_2 & C3⿻上丨 2 & $113.3(12)$ \\
\hline N3_2 & P1_2 & N4_2 & $98.7(4)$ & C41_2 & N12_2 & P3_ 2 & $115.0(8)$ \\
\hline $\mathrm{N} 42$ & $\mathrm{P} 12$ & N2 2 & $97.9(5)$ & $\mathrm{C} 41^{-} 2$ & N12-2 & C $4 \overline{5} 2$ & $105.8(10)$ \\
\hline $\mathrm{N} 5{ }^{-} 2$ & $\mathrm{P} 22$ & $\mathrm{~N} 6{ }^{-} 2$ & $114.4(4)$ & C45_2 & N12_2 & P3 $\overline{2}$ & $122.3(8)$ \\
\hline N5_2 & $\mathrm{P} 2 \_2$ & N7_2 & $112.3(4)$ & Ce1_2 & N13_2 & $\mathrm{K} 3$ & $94.5(3)$ \\
\hline N5_2 & $P 2 \_2$ & N8_2 & $119.9(5)$ & P4_ 2 & N13_2 & K3 & $122.5(5)$ \\
\hline N7_2 & $P 2 \_2$ & N6_2 & $105.7(5)$ & P4_2 & N13_2 & Ce1_2 & 141.2(6) \\
\hline $\mathrm{N} 8{ }^{-} 2$ & $\mathrm{P} 2{ }^{-} 2$ & $\mathrm{~N} 6{ }^{-} 2$ & $99.8(4)$ & $C 4 \overline{6} 2$ & N14_2 & P4_ 2 & $121.2(8)$ \\
\hline N8_2 & $\mathrm{P} 2 \_2$ & N7_2 & $102.9(5)$ & C50_2 & N14_2 & P4_2 & 123.2(9) \\
\hline N9_2 & P3 2 & N10_2 & $113.3(5)$ & C50_2 & N14_2 & C46__2 & $112.1(10)$ \\
\hline N9 2 & P3 2 & N11 2 & 119.7(6) & $\mathrm{C} 51^{-} 2$ & N15-2 & P4 $\overline{2}$ & $116.5(8)$ \\
\hline N9_2 & P3_2 & N12_2 & $115.2(5)$ & C55_2 & N15_2 & P4_2 & $114.0(8)$ \\
\hline N10_2 & P3_2 & N11_2 & $103.0(5)$ & C55_2 & N15_2 & C51_2 & $109.9(10)$ \\
\hline N10_2 & P3_2 & N12_2 & $104.4(5)$ & C56_2 & N16_2 & P4_ 2 & $118.1(7)$ \\
\hline N11-2 & P3 2 & $\mathrm{~N} 12^{-} 2$ & $99.2(5)$ & $\mathrm{C} 60^{-} 2$ & N16-2 & $\mathrm{P} 4-2$ & 117.1(8) \\
\hline N13-2 & $\mathrm{P} 4-2$ & $\mathrm{~N} 14^{-} 2$ & $113.8(5)$ & C60_2 & N16_2 & C5) 2 & $109.0(11)$ \\
\hline N13_2 & P4_2 & N15_2 & $115.1(5)$ & N2_ 2 & C1_ 2 & C2 2 & $113.4(10)$ \\
\hline N13_2 & P4_2 & N16_2 & $117.4(5)$ & C1_2 & C2_2 & C3_2 & 111.3(11) \\
\hline N14 2 & $\mathrm{P} 4{ }^{-} 2$ & N16-2 & $104.4(6)$ & $\mathrm{C} 22$ & $\mathrm{C} 3-2$ & $\mathrm{C} 4 \overline{2} 2$ & $109.9(11)$ \\
\hline N15 2 & $\mathrm{P} 4-2$ & $\mathrm{~N} 14^{-} 2$ & $104.5(5)$ & C5_2 & C4_2 & C3_2 & $111.2(11)$ \\
\hline N15_2 & P4_2 & N16_2 & $99.8(5)$ & N2_2 & C5_2 & C4_2 & $110.5(10)$ \\
\hline P1_ 2 & N1_2 & Ce1_2 & $176.5(7)$ & N3_2 & C6_2 & C7_2 & $110.7(9)^{\prime}$ \\
\hline
\end{tabular}




\begin{tabular}{|c|c|c|c|}
\hline Atom & Atom & Atom & Anglel $^{\circ}$ \\
\hline$\overline{C 622}$ & C7 2 & C8 2 & 109.3(9) \\
\hline C9_2 & C8_2 & C7_2 & 108.8(9) \\
\hline C10̄_2 & C9_2 & C8_2 & 111.4(9) \\
\hline N3 $\overline{2}$ & $\mathrm{C} 1 \overline{0} 2$ & $\mathrm{C}^{-} 2$ & 111.8(9) \\
\hline N4_2 & C11_2 & $\mathrm{C} 1 \overline{2} \_2$ & $109.5(9)$ \\
\hline C13̄2 & C12_2 & C11_2 & $112.3(10)$ \\
\hline C12_2 & C13_2 & C14_2 & 112.6(10) \\
\hline C15 2 & $\mathrm{C} 14^{-} 2$ & $\mathrm{C} 13^{-} 2$ & $111.4(9)$ \\
\hline N4__ & C15_2 & C14_2 & $110.9(9)$ \\
\hline N6_2 & C16_2 & C17_2 & 109.2(8) \\
\hline C1) 2 & C17_2 & C16_2 & 109.9(9) \\
\hline $\mathrm{C} 19^{-} 2$ & $\mathrm{C} 18^{-} 2$ & $\mathrm{C} 17^{-} 2$ & 111.4(9) \\
\hline C20_2 & C19_2 & C18_2 & $110.6(9)$ \\
\hline N6_2 & C20_2 & C19_2 & 109.6(9) \\
\hline N7 72 & $\mathrm{C} 21^{-} 2$ & $\mathrm{C} 22^{-} 2$ & $110.5(8)$ \\
\hline C2 $2 \overline{3} 2$ & $\mathrm{C} 222^{-2}$ & $\mathrm{C} 21^{-} 2$ & $110.4(9)$ \\
\hline C22_2 & C23_2 & C24_2 & 109.3(9) \\
\hline C23_2 & C24_2 & C25_2 & $110.0(8)$ \\
\hline N7_̄ & C25_2 & C24_2 & $110.0(8)$ \\
\hline N8_2 & $\mathrm{C} 26{ }^{-} 2$ & $\mathrm{C} 277^{-2}$ & $109.9(8)$ \\
\hline C2) 2 & C27_2 & C26_2 & $109.0(9)$ \\
\hline C29_2 & C28_2 & C27_2 & 108.7(9) \\
\hline $\mathrm{C} 3 \mathrm{O}^{-} 2$ & $\mathrm{C} 29^{-} 2$ & $\mathrm{C} 28^{-} 2$ & 111.3(9) \\
\hline N8_ $\overline{2}$ & $\mathrm{C} 30^{-} 2$ & $\mathrm{C} 29^{-} 2$ & 109.1(9) \\
\hline N10_2 & C31_2 & C32_2 & $110.0(10)$ \\
\hline C31_2 & C32_2 & C33_2 & $111.0(10)$ \\
\hline C32_2 & $\mathrm{C} 33^{-} 2$ & C34_2 & $109.5(10)$ \\
\hline C35_2 & C34_2 & C33_2 & $108.3(11)$ \\
\hline N10_2 & C35_2 & C34_2 & $112.3(11)$ \\
\hline N11-2 2 & $\mathrm{C} 36^{-} 2$ & $\mathrm{C} 37^{-} 2$ & $111.7(12)$ \\
\hline C38_2 & $\mathrm{C} 37^{-} 2$ & C36_2 & $109.4(10)$ \\
\hline C37_2 & C38_2 & C39_2 & $101.5(12)$ \\
\hline C38_2 & C39_2 & C40_2 & $109.2(10)$ \\
\hline N11_2 & C40_2 & C39_2 & $109.2(12)$ \\
\hline N12_2 & C41_2 & $\mathrm{C} 42^{-2} 2$ & $112.4(11)$ \\
\hline C43_2 & C42_2 & C41_2 & $109.5(12)$ \\
\hline C42_2 & C43_2 & C44_2 & $107.3(11)$ \\
\hline C43_2 & C44_2 & C45_2 & $110.9(12)$ \\
\hline N12_2 & C45_2 & C44_2 & $108.5(11)$ \\
\hline N14_2 & C46_2 & K3 & $89.3(7)$ \\
\hline N14_2 & C46_2 & C47_2 & $109.3(10)$ \\
\hline C47_2 & C46_2 & K3 & $86.2(7)$ \\
\hline C48_2 & C47_2 & C46_2 & $110.2(8)$ \\
\hline C49_2 & C48_2 & C47_2 & 109.8(9) \\
\hline C48_2 & C49_2 & C50_2 & $115.9(10)$ \\
\hline N14 2 & $\mathrm{C} 50^{-} 2$ & $\mathrm{C} 49^{-} 2$ & $113.9(11)$ \\
\hline N15_2 & C51_2 & C52_2 & $104.8(11)$ \\
\hline C53_2 & C52_2 & C51_2 & $109.6(10)$ \\
\hline C54_2 & C53_2 & C52_2 & $103.5(11)$ \\
\hline C55_2 & C54_2 & $\mathrm{C} 53^{-2}$ & $112.5(10)$ \\
\hline N15_2 & C55_2 & C54_2 & $117.7(12)$ \\
\hline N16_2 & C56_2 & C57_2 & $100.2(10)$ \\
\hline C58-2 & $\mathrm{C} 57^{-} 2$ & $\mathrm{C} 56^{-} 2$ & $106.9(9)$ \\
\hline C59-2 & C58_2 & $\mathrm{C} 57 \overline{2}$ & $109.4(11)$ \\
\hline C58_2 & C59_2 & C60_2 & $115.5(10)$ \\
\hline N16_2 & C60_2 & C59_2 & $112.3(10)$ \\
\hline N1_3 & Ce1_3 & K1 & $126.0(3)$ \\
\hline N1_3 & Ce1_3 & N5_3 & $113.1(4)$ \\
\hline N1_3 & Ce1_3 & N9_3 & $107.7(4)$ \\
\hline
\end{tabular}

\begin{tabular}{|c|c|c|c|}
\hline Atom & Atom & Atom & Angle $^{\circ}$ \\
\hline N13 & Ce13 & N13 3 & $112.2(4)$ \\
\hline N5_3 & Ce1_3 & K1 & $46.5(2)$ \\
\hline N5_3 & Ce1_3 & N13_3 & $91.5(3)$ \\
\hline N9_3 & Ce1_3 & $\mathrm{K} 1{ }^{-}$ & $126.3(3)$ \\
\hline N9_3 & Ce1_3 & N5_3 & 116.4(4) \\
\hline N9_3 & Ce1_3 & N13_3 & $115.5(4)$ \\
\hline N13_3 & Ce1_3 & K1 & $45.1(3)$ \\
\hline N1_ $\overline{3}$ & P1_̄ㄱ & N2_3 & $114.2(6)$ \\
\hline N1_3 & P1_3 & N3_3 & $111.8(5)$ \\
\hline N1_3 & P1_3 & N4_3 & $122.5(6)$ \\
\hline N3_3 & P1_3 & N2_3 & $108.6(5)$ \\
\hline N3_3 & P1_3 & N4_3 & $98.4(5)$ \\
\hline N4_3 & P1_3 & N2_3 & $99.5(5)$ \\
\hline N5_3 & P2_3 & N6_3 & 113.1(5) \\
\hline N5_3 & P2_3 & N7_3 & $112.5(5)$ \\
\hline N5_3 & $\mathrm{P} 23$ & N8_3 & $120.3(5)$ \\
\hline N7_3 & P2_3 & N6_3 & $106.3(5)$ \\
\hline N8_3 & P2_3 & N6_3 & $99.9(4)$ \\
\hline N8_3 & P2_3 & N7_3 & $103.0(5)$ \\
\hline N9_3 & P3_3 & N10_3 & $112.8(6)$ \\
\hline N9_3 & P3_3 & N11_3 & 119.2(6) \\
\hline N9_3 & P3_3 & N12_3 & 115.1(5) \\
\hline N10_3 & P3_3 & N11_3 & 102.1(5) \\
\hline N10_3 & $\mathrm{P} 3{ }^{-3}$ & N12_3 & $106.3(5)$ \\
\hline N11_3 & P3_3 & N12_3 & $99.5(5)$ \\
\hline N13_3 & P4_3 & N14_3 & $112.8(6)$ \\
\hline N13_3 & P4_3 & N15_3 & $117.3(6)$ \\
\hline N13_3 & P4_3 & N16_3 & 118.3(6) \\
\hline N15_3 & P4_3 & N14_3 & 104.3(5) \\
\hline N15_3 & P4_3 & N16_3 & $100.4(5)$ \\
\hline N16_3 & $\mathrm{P} 4 \_3$ & N14_3 & $101.6(5)$ \\
\hline P1_ 3 & N1_3 & Ce1_3 & $172.7(7)$ \\
\hline C1_3 & N2_3 & P1_3 & 119.3(8) \\
\hline C1_3 & N2_3 & C5_3 & 111.5(10) \\
\hline C5_3 & N2_3 & P1_3 & $112.8(7)$ \\
\hline C6_3 & N3_3 & P1_3 & $121.0(7)$ \\
\hline C6_3 & N3_3 & C10_3 & 113.5(9) \\
\hline$C 1 \overline{0} 3$ & N3 3 & $\mathrm{P} 1 \overline{3}$ & $113.9(7)$ \\
\hline C11_3 & N4_3 & P1_3 & $116.5(7)$ \\
\hline C11_3 & N4_3 & C15_3 & 110.6(9) \\
\hline C15_3 & N4_3 & P1_3 & 113.8(7) \\
\hline Ce1_3 & N5_3 & $\mathrm{K} 1$ & $94.3(3)$ \\
\hline P2_ 3 & N5_3 & $\mathrm{K} 1$ & $130.5(5)$ \\
\hline P2_3 & N5_3 & Ce1_3 & 133.8(5) \\
\hline C16_3 & N6_3 & P2_3 & $113.7(6)$ \\
\hline $\mathrm{C} 20^{-} 3$ & $\mathrm{~N} 6{ }^{-} 3$ & $\mathrm{P} 23$ & $123.0(7)$ \\
\hline C20_3 & N6_3 & C1)_3 & 110.3(8) \\
\hline C21_3 & N7_3 & P2_3 & $123.4(7)$ \\
\hline C21_3 & N7_3 & C25_3 & 112.3(8) \\
\hline $\mathrm{C} 25$ & N7 3 & $\mathrm{P} 2 \overline{3}$ & $122.4(7)$ \\
\hline C26_3 & N8_3 & P2_3 & $117.4(7)$ \\
\hline C26_3 & N8_3 & C30_3 & 108.8(8) \\
\hline C30_3 & N8_3 & P2_3 & 115.9(7) \\
\hline P3 3 & N9_3 & $\mathrm{Ce} \overline{1} 33$ & $165.9(7)$ \\
\hline C3̄1_3 & N10_3 & P3_ $\overline{3}$ & 118.9(8) \\
\hline C31_3 & N10_3 & C35_3 & 114.0(9) \\
\hline C35_3 & N10_3 & P3_ 3 & 123.2(8) \\
\hline C36_3 & N11_3 & P3_3 & 116.3(8) \\
\hline C40_3 & N11_3 & P3_3 & $118.2(7)$ \\
\hline
\end{tabular}




\begin{tabular}{|c|c|c|c|}
\hline Atom & Atom & Atom & Anglel $^{\circ}$ \\
\hline$\overline{C 403}$ & N113 & C36 3 & $112.4(10)$ \\
\hline C41_3 & N12_3 & P3_ $\overline{3}$ & $115.3(8)$ \\
\hline C41_3 & N12_3 & C45__3 & $108.0(10)$ \\
\hline C45_3 & N12_3 & P3_3 & $122.0(7)$ \\
\hline Ce1_3 & N13_3 & $\mathrm{K} 1$ & $95.6(3)$ \\
\hline P4_3 & N13_3 & $\mathrm{K} 1$ & 119.3(5) \\
\hline P4_3 & N13_3 & Ce1_3 & $143.9(6)$ \\
\hline C4⿳亠口冋_3 & N14_3 & P4_ $\overline{3}$ & 117.6(8) \\
\hline C50_3 & N14_3 & P4_3 & $119.7(8)$ \\
\hline C50_3 & N14_3 & C46_3 & $114.2(10)$ \\
\hline C51_3 & N15_3 & P4_3 & 116.8(8) \\
\hline C55_3 & N15_3 & P4_3 & 119.1(8) \\
\hline C55_3 & N15_3 & C51_3 & $109.7(11)$ \\
\hline C56_3 & N16_3 & P4_3 & $117.4(7)$ \\
\hline C60_3 & N16_3 & P4_3 & $114.6(7)$ \\
\hline C60-3 & N16_3 & C5 $5 \overline{6} 3$ & $109.0(10)$ \\
\hline N2_ 3 & C1_ 3 & C2_ 3 & $111.3(10)$ \\
\hline C1_3 & C2_3 & C3_3 & $110.3(10)$ \\
\hline C2_3 & C3_3 & C4_3 & $110.9(10)$ \\
\hline C5_3 & C4_3 & C3_3 & $111.9(10)$ \\
\hline N2_3 & C5_3 & C4_3 & $110.0(10)$ \\
\hline N3_3 & C6_3 & C7_3 & $110.0(9)$ \\
\hline C6_3 & C7_3 & C8_3 & 108.2(9) \\
\hline $\mathrm{CO}^{-} 3$ & $\mathrm{C} 8{ }^{-3}$ & $C 7-3$ & 109.6(9) \\
\hline C10_3 & C9_3 & C8_3 & $110.0(9)$ \\
\hline N3_3 & C10_3 & C9_3 & $110.4(9)$ \\
\hline N4_3 & C11_3 & C12_3 & 109.6(9) \\
\hline C1) 3 & C12_3 & C11_3 & $112.4(10)$ \\
\hline C12_3 & C13_3 & C14_3 & $111.7(10)$ \\
\hline C15_3 & C14_3 & C13_3 & $110.7(9)$ \\
\hline N4_ $\overline{3}$ & C15_3 & $\mathrm{C} 14+3$ & 110.2(9) \\
\hline N6_3 & C16_3 & C17_3 & 109.8(8) \\
\hline C16_3 & C17_3 & C18_3 & $110.4(8)$ \\
\hline C19_3 & C18_3 & C17_3 & $111.5(8)$ \\
\hline C18_3 & C19_3 & C20_3 & $109.8(9)$ \\
\hline N6_ 3 & C20_3 & C19_3 & 108.2(8) \\
\hline N7_3 & C21_3 & C22_3 & $110.4(9)$ \\
\hline C23_3 & C22_3 & C21_3 & $110.4(9)$ \\
\hline C22_3 & C23_3 & C24_3 & 109.3(9) \\
\hline C23_3 & C24_3 & C25_3 & $110.7(8)$ \\
\hline N7_3 & C25_3 & C24_3 & 109.3(9) \\
\hline N8_3 & C26_3 & C27_3 & 109.5(8) \\
\hline C28_3 & C27_3 & C26_3 & 107.4(8) \\
\hline C29_3 & C28_3 & C27_3 & $108.0(9)$ \\
\hline C30_3 & C29_3 & C28_3 & 109.9(9) \\
\hline N8_3 & C30_3 & C29_3 & 108.6(9) \\
\hline N10_3 & C31_3 & C32_3 & $111.6(10)$ \\
\hline C31_3 & C32_3 & C33_3 & $111.5(10)$ \\
\hline C32_3 & C33_3 & C34_3 & $110.0(10)$ \\
\hline C35_3 & C34_3 & C33_3 & $108.7(10)$ \\
\hline N10_3 & C35_3 & C34_3 & $113.8(10)$ \\
\hline N11_3 & C36_3 & C37_3 & $112.6(10)$ \\
\hline C38_3 & C37_3 & C36_3 & $110.5(5)$ \\
\hline C37_3 & C38_3 & C39_3 & $102.3(10)$ \\
\hline C38_3 & C39_3 & C40_3 & $110.5(5)$ \\
\hline N11_3 & C40_3 & C39_3 & $110.5(11)$ \\
\hline N12_3 & C41_3 & C42_3 & $112.7(10)$ \\
\hline C43_3 & C42_3 & C41_3 & $109.5(10)$ \\
\hline C42_3 & C43_3 & C44_3 & $106.8(10)$ \\
\hline
\end{tabular}

\begin{tabular}{|c|c|c|c|}
\hline Atom & Atom & Atom & Angle $^{\circ}$ \\
\hline C43 3 & C44 3 & C45 3 & $110.5(10)$ \\
\hline N12_3 & C45_3 & C44_3 & 109.8(10) \\
\hline N14_3 & C46_3 & C47_3 & $112.5(10)$ \\
\hline C48_3 & C47_3 & C46_3 & $111.7(5)$ \\
\hline C49_3 & C48_3 & C47_3 & $108.8(10)$ \\
\hline C48_3 & C49_3 & C50_3 & $111.8(5)$ \\
\hline N14_3 & C50_3 & C49_3 & $113.0(11)$ \\
\hline N153 3 & $\mathrm{C} 51^{-} 3$ & $\mathrm{C} 52^{-} 3$ & 105.1(11) \\
\hline C53_3 & C52_3 & C51_3 & $110.1(5)$ \\
\hline C54_3 & C53_3 & C52_3 & 103.2(11) \\
\hline C53_3 & C54_3 & C55_3 & 111.7(6) \\
\hline N15_3 & C55_3 & C54_3 & $116.6(12)$ \\
\hline C57_3 & C56_3 & N16_3 & 103.4(10) \\
\hline C58_3 & C57_3 & C56_3 & $110.6(5)$ \\
\hline C59_3 & C58_3 & C57_3 & 108.2(10) \\
\hline C58_3 & C59-3 & $\mathrm{C} 60^{-} 3$ & $111.5(5)$ \\
\hline N16_3 & C60_3 & C59_3 & $110.0(10)$ \\
\hline C2_7 & 01_7 & K3 & $92.6(11)$ \\
\hline C2_7 & 01_7 & C3_7 & $113.0(6)$ \\
\hline $\mathrm{C} 3-7$ & O1_7 & $\mathrm{K} 3$ & $126.7(11)$ \\
\hline 01_7 & $\mathrm{C} 2 \_7$ & K3 & $60.9(9)$ \\
\hline 01_7 & C2_7 & C1_7 & $107.9(5)$ \\
\hline $\mathrm{C}^{-} 7$ & $\mathrm{C} 2^{-7} 7$ & $\mathrm{~K}^{-}$ & $131.5(17)$ \\
\hline $01-7$ & $\mathrm{C} 3-7$ & C4_7 & $107.4(5)$ \\
\hline C2_6 & 01_6 & $\mathrm{K}^{-}$ & 120.1(7) \\
\hline C2_6 & 01_6 & C3_6 & $112.2(6)$ \\
\hline C3-6 & 01_6 & $\mathrm{K}^{-}$ & $122.5(6)$ \\
\hline 01_6 & C2_6 & C1_6 & $107.9(5)$ \\
\hline O1_6 & C3_6 & C4_6 & $107.7(5)$ \\
\hline C3_6 & C4_6 & $\mathrm{K} 3$ & $85.0(5)$ \\
\hline C2_8 & 01_8 & $\mathrm{K} 1$ & $131.2(10)$ \\
\hline C2_-8 & 01_8 & C3_8 & $113.4(6)$ \\
\hline C3_8 & 01_8 & $\mathrm{K} 1$ & $104.0(11)$ \\
\hline 01_8 & C2_-8 & C1_8 & $107.7(5)$ \\
\hline 01_8 & $\mathrm{C} 3-8$ & $\mathrm{~K} 1^{-}$ & $52.1(8)$ \\
\hline 01_8 & C3_8 & C4_8 & $107.5(5)$ \\
\hline C4_8 & C3_8 & $\mathrm{K} 1$ & $92.0(13)$ \\
\hline $\mathrm{N} 1-1$ & $\mathrm{Ce} \overline{1} 1$ & K2 & $130.6(3)$ \\
\hline N1_1 & Ce1_1 & N5_1 & 111.3(3) \\
\hline N1_1 & Ce1_1 & N9_1 & 106.9(3) \\
\hline N1_1 & Ce1_1 & $\mathrm{N} 1 \overline{3} \_1$ & $112.5(3)$ \\
\hline N5 1 & $\mathrm{Ce} 1-1$ & K2 & $47.1(2)$ \\
\hline N9_1 & Ce1_1 & K2 & $122.5(3)$ \\
\hline N9_1 & Ce1_1 & N5_1 & 116.1(3) \\
\hline N9_1 & Ce1_1 & N13_1 & 117.3(3) \\
\hline $\mathrm{N} 1 \overline{3} 1$ & $\mathrm{Ce} 1-1$ & $\mathrm{~K} 2$ & $46.3(2)$ \\
\hline N13_1 & Ce1_1 & N5_1 & $92.4(3)$ \\
\hline N1_ì & P1_1 & N2_1 & 113.0(5) \\
\hline N1_1 & $\mathrm{P} 1{ }^{-} 1$ & N3_1 & 113.0(5) \\
\hline N1_1 & $\mathrm{P} 1$ & $\mathrm{~N} 4 \_1$ & $122.2(5)$ \\
\hline N3_1 & P1_1 & N2_1 & $109.0(5)$ \\
\hline N3_1 & $\mathrm{P} 1{ }_{-1}^{-1}$ & N4_1 & $99.4(5)$ \\
\hline $\mathrm{N}^{-}-1$ & $\mathrm{P} 1^{-} 1$ & $\mathrm{~N} 2-1$ & $98.5(5)$ \\
\hline N5_1 & $\mathrm{P} 21$ & N6 1 & $113.4(5)$ \\
\hline N5_1 & P2_1 & N7_1 & $113.5(5)$ \\
\hline N5_1 & $\mathrm{P} 2-1$ & N8_1 & $120.3(5)$ \\
\hline N7_1 & P2_1 & N6_1 & 106.4(5) \\
\hline N7_-1 & $\mathrm{P} 2 \_1$ & N8_1 & $101.5(5)$ \\
\hline N8_1 & P2_1 & N6_1 & $99.8(4)$ \\
\hline
\end{tabular}




\begin{tabular}{|c|c|c|c|}
\hline Atom & Atom & Atom & Angle ${ }^{\circ}$ \\
\hline $\bar{N} 91$ & P3 1 & N10 1 & $113.6(5)$ \\
\hline N9_1 & P3_1 & N11_1 & $117.6(6)$ \\
\hline N9_1 & P3_1 & N12_1 & $117.5(5)$ \\
\hline N10_1 & P3_1 & N11_1 & $102.7(6)$ \\
\hline N10_1 & P3_1 & N12_1 & $104.6(5)$ \\
\hline N11_1 & P3_1 & N12_1 & $98.5(5)$ \\
\hline N13_1 & $\mathrm{P} 4{ }^{-} 1$ & N14_1 & $112.9(5)$ \\
\hline N13_1 & P4_1 & N15_1 & 117.6(5) \\
\hline N13_1 & P4_1 & N16_1 & $115.2(5)$ \\
\hline N15_1 & P4_1 & N14_1 & $106.2(5)$ \\
\hline N15_1 & P4_1 & N16_1 & $99.8(5)$ \\
\hline N16_1 & P4_1 & N14_1 & $103.5(5)$ \\
\hline P1_-1 & N1_1 & Ce1_1 & $173.7(7)$ \\
\hline C1_1 & N2_1 & P1_1 & $124.3(8)$ \\
\hline C1_1 & N2_1 & C5_1 & $113.4(10)$ \\
\hline C5 5 & $\mathrm{~N} 2$ & $\mathrm{P} 1-1$ & $115.8(7)$ \\
\hline C6_1 & N3_1 & P1_1 & $121.5(7)$ \\
\hline C6_1 & N3_1 & C10_1 & 111.4(9) \\
\hline C10_1 & N3_1 & P1_1 & 115.7(7) \\
\hline C11_1 & N4_1 & P1_1 & $114.6(7)$ \\
\hline C11_1 & N4_1 & C15_1 & 109.8(9) \\
\hline C15_1 & N4_1 & P1_1 & $115.1(7)$ \\
\hline Ce1_1 & N5_1 & $\mathrm{K} 2$ & $93.4(3)$ \\
\hline P2_- & N5_1 & $\mathrm{K} 2$ & 127.1(4) \\
\hline P2_1 & N5_1 & Ce1_1 & $137.4(5)$ \\
\hline C16_1 & N6_1 & P2_1 & $113.6(6)$ \\
\hline C20_1 & N6_1 & P2_1 & $124.2(7)$ \\
\hline C20_1 & N6_1 & C16_1 & $110.0(8)$ \\
\hline C21_1 & N7_1 & P2_1 & $123.9(7)$ \\
\hline C21_1 & N7_-1 & C25_1 & $113.0(8)$ \\
\hline C25_1 & N7_-1 & P2_-̄ & $121.9(7)$ \\
\hline C26_1 & N8_1 & P2_1 & $115.9(6)$ \\
\hline C26_1 & N8_1 & C30_1 & 107.7(8) \\
\hline C30_1 & N8_1 & P2_1 & 115.3(6) \\
\hline P3_1 & N9_1 & Ce1_1 & 167.2(7) \\
\hline C31__1 & N10__1 & P3_ & $118.9(8)$ \\
\hline C31_1 & N10_1 & C35_1 & $113.7(10)$ \\
\hline C35_1 & N10_1 & P3_1 & $121.8(8)$ \\
\hline C36_1 & N11_1 & P3_1 & $117.5(8)$ \\
\hline C40_1 & N11_1 & P3_1 & $120.3(8)$ \\
\hline C40_1 & N11_1 & C36_1 & 111.2(11) \\
\hline C41_1 & N12_1 & P3_1 & 117.1(8) \\
\hline C41_1 & N12_1 & C45_1 & $107.9(10)$ \\
\hline C45_1 & N12_1 & P3_1 & $119.9(8)$ \\
\hline Ce1_1 & N13_1 & K2 & $94.3(3)$ \\
\hline P4_1 & N13_1 & K2 & $122.9(5)$ \\
\hline P4_1 & N13_1 & Ce1_1 & $139.5(5)$ \\
\hline C46_1 & N14_1 & P4_1 & $121.3(8)$ \\
\hline C50_1 & N14_1 & P4_1 & $120.8(8)$ \\
\hline C50_1 & N14_1 & C46_1 & 117.2(9) \\
\hline C51_1 & N15_1 & P4_1 & $119.6(8)$ \\
\hline C55_1 & N15_1 & P4_1 & 115.2(8) \\
\hline C55_1 & N15_1 & C51_1 & 108.2(10) \\
\hline C56_1 & N16_1 & P4_1 & $124.5(7)$ \\
\hline C60_1 & N16_1 & P4_1 & $115.8(7)$ \\
\hline C60_1 & N16_1 & C56̄_1 & $109.7(10)$ \\
\hline N2_1 & C1_1 & C2_1 & $112.1(10)$ \\
\hline C1_1 & C2_1 & C3_1 & $111.2(11)$ \\
\hline C2_1 & C3_1 & C4_1 & $111.4(10)$ \\
\hline
\end{tabular}

\begin{tabular}{|c|c|c|c|}
\hline Atom & Atom & Atom & Angle $^{\circ}$ \\
\hline$\overline{C 5} 1$ & C4 1 & C3 1 & $111.2(10)$ \\
\hline N2_-1 & C5_1 & C4_1 & 108.4(10) \\
\hline N3_1 & C6_1 & $\mathrm{C} 7{ }_{-1}^{-1}$ & $110.9(9)$ \\
\hline C6_1 & C7_1 & C8_1 & 109.9(9) \\
\hline C9_1 & C8_1 & C7_1 & 109.1(9) \\
\hline C10_1 & C9_1 & C8_1 & $110.9(10)$ \\
\hline N3_1 & C10_1 & C9_1 & 112.6(9) \\
\hline N4_1 & C11_1 & C12__1 & 109.2(9) \\
\hline C13 1 & C12_1 & C11_1 & $112.7(10)$ \\
\hline C12_1 & C13_1 & C14_1 & 111.2(10) \\
\hline C15_1 & C14_1 & C13_1 & $110.5(10)$ \\
\hline N4_1 & C15_1 & C14_1 & $110.0(9)$ \\
\hline N6_1 & C16_1 & C17_1 & $1(8)$ \\
\hline C16_1 & C17_1 & C18_1 & (9) \\
\hline C19_1 & C18_1 & C17_1 & 11 \\
\hline C18_1 & $\mathrm{C} 19^{-1}$ & $\mathrm{C} 20^{-}-1$ & \\
\hline N6_- & C20_1 & C19_1 & \\
\hline N7_1 & C21_1 & C22_1 & $110.0(9)$ \\
\hline C23_1 & C22_1 & C21_1 & 110.8(9) \\
\hline $\mathrm{C} 22^{-} 1$ & $\mathrm{C} 23^{-1}$ & $\mathrm{C} 244^{-} 1$ & 4(9) \\
\hline C23_1 & C24_1 & C25_1 & \\
\hline N7_1 & C25_1 & C24_1 & \\
\hline N8_-1 & C26_1 & C27_1 & \\
\hline $\mathrm{C} 2 \overline{6} \_1$ & $\mathrm{C} 27^{-1}$ & $\mathrm{C} 28-1$ & (8) \\
\hline C29_1 & C28_1 & C27_1 & 107.8(9) \\
\hline C30_1 & C29_1 & C28_1 & $3(9)$ \\
\hline N8_- & C30_1 & C29_1 & (9) \\
\hline N10_1 & & C32_1 & (10) \\
\hline C31_1 & 2 & C33_1 & \\
\hline C32_1 & C33_1 & C34_1 & \\
\hline C35_1 & C34_1 & C33_1 & (11) \\
\hline N10_1 & C35_1 & C34_1 & 1(11) \\
\hline N11_1 & C36_1 & C37_1 & $112.0(12)$ \\
\hline C38_1 & C37_1 & C36_1 & 110.3(10) \\
\hline C $37^{-1}$ & C $38-1$ & $\mathrm{C} 39^{-1}$ & $6(12)$ \\
\hline 1 & & $0 \_1$ & (10) \\
\hline 1_1 & C40_1 & 39_1 & 12) \\
\hline N12_1 & C41_1 & C42_1 & (11) \\
\hline C43_1 & C42_1 & C41_1 & $2(11)$ \\
\hline C42_1 & C43_1 & C44_1 & $9(11)$ \\
\hline C43_1 & C44_1 & C45_1 & f(11) \\
\hline N12_1 & C45_1 & C44_1 & $9(10)$ \\
\hline N14_1 & C46_1 & C47_1 & (10) \\
\hline C48_1 & C47_1 & C46_1 & \\
\hline C49_1 & C48_1 & C47_1 & 10 \\
\hline C48_1 & C49_1 & C50_1 & (9) \\
\hline N14_1 & C50_1 & C49_1 & $5(11)$ \\
\hline N15_1 & C51_1 & C52_1 & 104.6(11) \\
\hline C53_1 & C52_1 & C51_1 & 109.8(10) \\
\hline C54_1 & C53_1 & C52_1 & 103.7(11) \\
\hline C53_1 & C54_1 & C55_1 & 110.6(9) \\
\hline N15_1 & C55_1 & C54_1 & $115.4(11)$ \\
\hline N16_1 & C56_1 & C57_1 & 103.2(10) \\
\hline C58_1 & C57_1 & C56_1 & 111.7(10) \\
\hline C5s & C58_1 & C57_1 & 109.3(10) \\
\hline C58_1 & C59_1 & C60_1 & 111.0(9) \\
\hline N16_1 & C60_1 & C59_1 & 108.0(10) \\
\hline C2 $2 \overline{4}$ & $01+\overline{4}$ & K2 & $119.4(7)$ \\
\hline C2 44 & O1_4 & C3_4 & $113.0(6)$ \\
\hline
\end{tabular}




\begin{tabular}{llll}
\hline Atom & Atom & Atom & \multicolumn{1}{c}{ Angle $^{\circ}$} \\
\hline C3_4 & O1_4 & K2 & $126.7(6)$ \\
C2_4 & C1_4 & K2 & $86.7(6)$ \\
O1_4 & C2_4 & C1_4 & $107.9(5)$ \\
O1_4 & C3_4 & C4_4 & $107.5(5)$ \\
C2_5 & O1_5 & K2 & $112.9(8)$ \\
C2_5 & O1_5 & C3_5 & $113.3(6)$ \\
C3_5 & O1_5 & K2 & $129.9(9)$
\end{tabular}

\begin{tabular}{llll}
\hline Atom & Atom & Atom & Angle $^{\circ}$ \\
\hline O1_5 & C2_5 & C1_5 & $107.8(5)$ \\
O1_5 & C3_5 & C4_5 & $107.4(5)$ \\
C2_9 & O1_9 & K1 & $128.3(9)$ \\
C2_9 & O1_9 & C3_9 & $112.7(6)$ \\
C3_9 & O1_9 & K1 & $112.9(10)$ \\
O1_9 & C2_9 & C1_9 & $107.7(5)$ \\
O1_9 & C3_9 & C4_9 & $107.6(5)$
\end{tabular}

Table S10: Bond Lengths in $\AA$ for 3-Ce-K222.

\begin{tabular}{|c|c|c|c|c|c|}
\hline Atom & Atom & Length/Å & Atom & Atom & Length/Å \\
\hline$\overline{\mathrm{Ce} 1}$ & N1 & $2.329(2)$ & $\overline{\mathrm{N} 6}$ & C16 & $1.449(4)$ \\
\hline Ce1 & N5 & $2.293(3)$ & N6 & $\mathrm{C} 20$ & $1.458(4)$ \\
\hline Ce1 & N9 & $2.324(2)$ & N7 & $\mathrm{C} 21$ & $1.460(4)$ \\
\hline Ce1 & N13 & $2.339(2)$ & N7 & $\mathrm{C} 25$ & $1.461(4)$ \\
\hline K1 & 01 & $2.783(2)$ & N8 & C26 & $1.466(4)$ \\
\hline K1 & $\mathrm{O} 2$ & $2.759(2)$ & N8 & C30 & $1.459(5)$ \\
\hline K1 & $\mathrm{O} 3$ & $2.826(2)$ & N10 & C31 & $1.464(4)$ \\
\hline K1 & $\mathrm{O} 4$ & $2.840(2)$ & N10 & C35 & $1.463(4)$ \\
\hline K1 & O5 & $2.878(3)$ & N11 & C36 & $1.482(5)$ \\
\hline K1 & N17 & $2.994(3)$ & N11 & C40 & $1.482(4)$ \\
\hline K1 & N18 & $3.055(3)$ & $\mathrm{N} 12$ & C41 & $1.465(4)$ \\
\hline K1 & 019 & $2.817(3)$ & $\mathrm{N} 12$ & $\mathrm{C} 45$ & $1.461(4)$ \\
\hline $\mathrm{P} 1$ & $\mathrm{~N} 1$ & $1.532(3)$ & N14 & C46 & $1.475(4)$ \\
\hline P1 & N3 & $1.691(3)$ & N14 & C50 & $1.468(3)$ \\
\hline P1 & N4 & $1.700(2)$ & N16 & C56 & $1.452(4)$ \\
\hline P1 & N2 1 & $1.703(2)$ & N16 & C60 & $1.461(4)$ \\
\hline $\mathrm{P} 2$ & N5 & $1.530(3)$ & N17 & C64 & $1.478(4)$ \\
\hline $\mathrm{P} 2$ & N6 & $1.708(3)$ & N17 & C65 & $1.463(5)$ \\
\hline $\mathrm{P} 2$ & N7 & $1.685(3)$ & N17 & $\mathrm{C} 78$ & $1.473(5)$ \\
\hline P2 & N8 & $1.679(3)$ & N18 & C70 & $1.475(4)$ \\
\hline P3 & N9 & $1.535(3)$ & N18 & C71 & $1.456(5)$ \\
\hline P3 & N10 & $1.698(3)$ & N18 & C73 & $1.477(4)$ \\
\hline P3 & N11 & $1.709(2)$ & 019 & C76 & $1.448(5)$ \\
\hline P3 & N12 & $1.700(3)$ & 019 & C77 & $1.416(4)$ \\
\hline P4 & N13 & $1.522(2)$ & C6 & C7 & $1.502(5)$ \\
\hline P4 & N14 & $1.703(2)$ & $\mathrm{C} 7$ & $\mathrm{C} 8$ & $1.530(5)$ \\
\hline P4 & N16 & $1.695(2)$ & $\mathrm{C} 8$ & $\mathrm{C9}$ & $1.540(6)$ \\
\hline P4 & N2 3 & $1.680(4)$ & C9 & C10 & $1.500(6)$ \\
\hline P4 & N2_2 & $1.724(3)$ & C11 & C12 & $1.491(5)$ \\
\hline 01 & C61 & $1.423(4)$ & C12 & C13 & $1.528(4)$ \\
\hline $\mathrm{O} 1$ & C72 & $1.414(3)$ & C13 & C14 & $1.547(5)$ \\
\hline $\mathrm{O} 2$ & C62 & $1.435(3)$ & C14 & C15 & $1.524(5)$ \\
\hline $\mathrm{O} 2$ & C63 & $1.435(3)$ & C16 & C17 & $1.517(5)$ \\
\hline $\mathrm{O} 3$ & C66 & $1.407(5)$ & C17 & C18 & $1.530(5)$ \\
\hline $\mathrm{O} 3$ & $\mathrm{C} 67$ & $1.428(4)$ & C18 & C19 & $1.535(6)$ \\
\hline $\mathrm{O} 4$ & C68 & $1.414(4)$ & C19 & $\mathrm{C} 20$ & $1.500(6)$ \\
\hline O4 & C69 & $1.419(4)$ & $\mathrm{C} 21$ & $\mathrm{C} 22$ & $1.515(6)$ \\
\hline O5 & C74 & $1.431(5)$ & $\mathrm{C} 22$ & $\mathrm{C} 23$ & $1.500(8)$ \\
\hline O5 & C75 & $1.438(5)$ & $\mathrm{C} 23$ & $\mathrm{C} 24$ & $1.518(8)$ \\
\hline $\mathrm{O} 6$ & C79 & $1.416(3)$ & $\mathrm{C} 24$ & $\mathrm{C} 25$ & 1.515(6) \\
\hline $\mathrm{O} 6$ & C81 & $1.412(3)$ & C26 & $\mathrm{C} 27$ & $1.499(6)$ \\
\hline N3 & $\mathrm{C} 6$ & $1.479(4)$ & $\mathrm{C} 27$ & $\mathrm{C} 28$ & $1.555(6)$ \\
\hline N3 & C10 & $1.462(4)$ & C28 & $\mathrm{C} 29$ & $1.522(7)$ \\
\hline N4 & C11 & $1.481(4)$ & C29 & C30 & $1.521(7)$ \\
\hline \multirow[t]{2}{*}{ N4 } & C15 & $1.465(4)$ & C31 & C32 & $1.529(5)$ \\
\hline & & & C32 & C33 & $1.525(5)$ \\
\hline
\end{tabular}




\begin{tabular}{lll}
\hline Atom & Atom & Length/A \\
\hline C33 & C34 & $1.549(5)$ \\
C34 & C35 & $1.515(5)$ \\
C36 & C37 & $1.537(5)$ \\
C37 & C38 & $1.517(6)$ \\
C38 & C39 & $1.515(6)$ \\
C39 & C40 & $1.511(4)$ \\
C41 & C42 & $1.531(6)$ \\
C42 & C43 & $1.524(6)$ \\
C43 & C44 & $1.524(6)$ \\
C44 & C45 & $1.528(5)$ \\
C46 & C47 & $1.520(5)$ \\
C47 & C48 & $1.536(5)$ \\
C48 & C49 & $1.533(5)$ \\
C49 & C50 & $1.508(4)$ \\
C56 & C57 & $1.527(5)$ \\
C57 & C58 & $1.542(5)$ \\
C58 & C59 & $1.508(5)$ \\
C59 & C60 & $1.516(4)$ \\
C61 & C62 & $1.502(4)$ \\
C63 & C64 & $1.504(5)$ \\
C65 & C66 & $1.531(6)$ \\
C67 & C68 & $1.511(5)$ \\
C69 & C70 & $1.497(5)$ \\
C71 & C72 & $1.519(5)$ \\
& &
\end{tabular}

\begin{tabular}{lll}
\hline Atom & Atom & Length/A \\
\hline C73 & C74 & $1.474(6)$ \\
C75 & C76 & $1.486(6)$ \\
C77 & C78 & $1.495(7)$ \\
C79 & C80 & $1.510(6)$ \\
C81 & C82 & $1.539(6)$ \\
N2_1 & C1_1 & $1.470(3)$ \\
N2_1 & C5_1 & $1.471(2)$ \\
C1_1 & C2_1 & $1.518(4)$ \\
C2_1 & C3_1 & $1.524(4)$ \\
C3_1 & C4_1 & $1.518(4)$ \\
C4_1 & C5_1 & $1.522(4)$ \\
N2_3 & C1_3 & $1.468(3)$ \\
N2_3 & C5_3 & $1.468(3)$ \\
C1_3 & C2_3 & $1.517(4)$ \\
C2_3 & C3_3 & $1.524(4)$ \\
C3_3 & C4_3 & $1.519(4)$ \\
C4_3 & C5_3 & $1.520(4)$ \\
N2_2 & C1_2 & $1.469(3)$ \\
N2_2 & C5_2 & $1.467(3)$ \\
C1_2 & C2_2 & $1.518(4)$ \\
C2_2 & C3_2 & $1.523(4)$ \\
C3_2 & C4_2 & $1.518(4)$ \\
C4_2 & C5_2 & $1.519(4)$
\end{tabular}

Table S11: Bond Angles in ${ }^{\circ}$ for 3-Ce-K222.

\begin{tabular}{|c|c|c|c|c|c|c|c|}
\hline Atom & Atom & Atom & Angle $^{\circ}$ & Atom & Atom & Atom & Angle $^{\circ}$ \\
\hline$\overline{\mathrm{N} 1}$ & $\mathrm{Ce} 1$ & N13 & $106.88(9)$ & $\overline{\mathrm{O} 4}$ & $\mathrm{~K} 1$ & N18 & $59.64(7)$ \\
\hline N5 & $\mathrm{Ce} 1$ & $\mathrm{~N} 1$ & $108.05(10)$ & 05 & $\mathrm{~K} 1$ & N17 & 120.55(9) \\
\hline N5 & Ce1 & N9 & $104.55(10)$ & 05 & $\mathrm{~K} 1$ & N18 & $58.60(8)$ \\
\hline N5 & $\mathrm{Ce} 1$ & N13 & $110.79(11)$ & N17 & $\mathrm{K} 1$ & N18 & 178.76(9) \\
\hline N9 & Ce1 & $\mathrm{N} 1$ & $113.55(11)$ & O19 & $\mathrm{K} 1$ & $\mathrm{O} 3$ & $99.87(8)$ \\
\hline N9 & $\mathrm{Ce} 1$ & N13 & 112.97(9) & O19 & $\mathrm{K} 1$ & $\mathrm{O} 4$ & $121.81(7)$ \\
\hline 01 & $\mathrm{~K} 1$ & $\mathrm{O} 3$ & $121.36(7)$ & O19 & $\mathrm{K} 1$ & $\mathrm{O} 5$ & $59.92(8)$ \\
\hline 01 & $\mathrm{~K} 1$ & O4 & $97.43(6)$ & O19 & $\mathrm{K} 1$ & N17 & $61.27(9)$ \\
\hline $\mathrm{O} 1$ & $\mathrm{~K} 1$ & O5 & $94.76(7)$ & 019 & $\mathrm{~K} 1$ & N18 & $117.74(8)$ \\
\hline 01 & $\mathrm{~K} 1$ & N17 & $120.72(7)$ & N1 & $\mathrm{P} 1$ & N3 & 114.86(15) \\
\hline 01 & $\mathrm{~K} 1$ & N18 & $60.47(7)$ & N1 & $\mathrm{P} 1$ & N4 & $112.28(13)$ \\
\hline 01 & $\mathrm{~K} 1$ & 019 & $134.09(8)$ & N1 & $\mathrm{P} 1$ & N2_1 & $120.45(14)$ \\
\hline $\mathrm{O} 2$ & K1 & O1 & $61.43(6)$ & N3 & P1 & N4 & $106.52(15)$ \\
\hline $\mathrm{O} 2$ & $\mathrm{~K} 1$ & O3 & $97.07(7)$ & N3 & $\mathrm{P} 1$ & N2_1 & $101.07(12)$ \\
\hline $\mathrm{O} 2$ & $\mathrm{~K} 1$ & O4 & $136.70(7)$ & N4 & $\mathrm{P} 1$ & $\mathrm{~N} 2-1$ & $99.74(12)$ \\
\hline $\mathrm{O} 2$ & $\mathrm{~K} 1$ & O5 & $120.05(8)$ & N5 & $\mathrm{P} 2$ & $\mathrm{~N} 6$ & $119.80(15)$ \\
\hline $\mathrm{O} 2$ & K1 & N17 & $59.81(7)$ & N5 & $\mathrm{P} 2$ & N7 & $113.87(16)$ \\
\hline $\mathrm{O} 2$ & $\mathrm{~K} 1$ & N18 & $121.31(7)$ & N5 & $\mathrm{P} 2$ & N8 & $112.57(16)$ \\
\hline $\mathrm{O} 2$ & $\mathrm{~K} 1$ & 019 & $96.77(7)$ & N7 & $\mathrm{P} 2$ & N6 & $97.60(14)$ \\
\hline $\mathrm{O} 3$ & $\mathrm{~K} 1$ & $\mathrm{O} 4$ & $60.22(6)$ & N8 & $\mathrm{P} 2$ & N6 & $101.23(13)$ \\
\hline $\mathrm{O} 3$ & $\mathrm{~K} 1$ & O5 & $138.17(8)$ & N8 & $\mathrm{P} 2$ & N7 & $110.22(14)$ \\
\hline $\mathrm{O} 3$ & $\mathrm{~K} 1$ & N17 & $60.59(8)$ & N9 & P3 & N10 & $113.51(15)$ \\
\hline $\mathrm{O} 3$ & $\mathrm{~K} 1$ & N18 & $119.30(7)$ & N9 & P3 & N11 & $121.48(14)$ \\
\hline $\mathrm{O} 4$ & $\mathrm{~K} 1$ & O5 & $97.61(7)$ & N9 & P3 & N12 & $113.88(14)$ \\
\hline $\mathrm{O} 4$ & $\mathrm{~K} 1$ & N17 & $120.05(8)$ & N10 & P3 & N11 & $100.99(13)$ \\
\hline
\end{tabular}




\begin{tabular}{|c|c|c|c|c|c|c|c|}
\hline Atom & Atom & Atom & Angle $^{\circ}$ & Atom & Atom & Atom & Angle $^{\circ}$ \\
\hline$\overline{N 10}$ & P3 & N12 & $106.68(13)$ & $\overline{\mathrm{P} 4}$ & N13 & $\mathrm{Ce} 1$ & $153.04(15)$ \\
\hline N12 & P3 & N11 & $98.20(13)$ & C46 & N14 & P4 & $114.56(18)$ \\
\hline N13 & P4 & N14 & $120.64(13)$ & C50 & N14 & P4 & $114.34(18)$ \\
\hline N13 & P4 & N16 & $114.32(13)$ & C50 & N14 & C46 & $109.8(2)$ \\
\hline N13 & P4 & N2_3 & $115.34(19)$ & C56 & N16 & P4 & $125.54(19)$ \\
\hline N13 & P4 & N2_2 & $110.08(16)$ & C56 & N16 & C60 & $113.9(2)$ \\
\hline N14 & P4 & N2_2 & $100.3(2)$ & $\mathrm{C} 60$ & N16 & $\mathrm{P} 4$ & $117.4(2)$ \\
\hline N16 & P4 & $\mathrm{N} 1 \overline{4}$ & $98.77(12)$ & C64 & N17 & $\mathrm{K} 1$ & $110.12(17)$ \\
\hline N16 & P4 & N2_2 & $111.6(2)$ & C65 & N17 & $\mathrm{K} 1$ & $108.9(2)$ \\
\hline N2_3 & P4 & $\mathrm{N} 14$ & $99.6(3)$ & C65 & N17 & C64 & $108.4(3)$ \\
\hline $\mathrm{N} 23$ & P4 & N16 & $105.7(3)$ & C65 & N17 & C78 & $112.0(3)$ \\
\hline C6̄i & 01 & $\mathrm{~K} 1$ & $111.57(15)$ & C78 & N17 & $\mathrm{K} 1$ & $106.9(2)$ \\
\hline C72 & 01 & $\mathrm{~K} 1$ & $119.07(18)$ & C78 & N17 & C64 & $110.5(3)$ \\
\hline $\mathrm{C} 72$ & 01 & C61 & $109.7(2)$ & C70 & N18 & $\mathrm{K} 1$ & $109.27(17)$ \\
\hline C62 & $\mathrm{O} 2$ & $\mathrm{~K} 1$ & $116.41(16)$ & C70 & N18 & C73 & $109.2(3)$ \\
\hline C63 & $\mathrm{O} 2$ & K1 & $121.57(17)$ & C71 & N18 & K1 & $106.96(18)$ \\
\hline C63 & $\mathrm{O} 2$ & C62 & $111.4(2)$ & C71 & N18 & C70 & $110.5(3)$ \\
\hline C66 & O3 & $\mathrm{K} 1$ & $118.2(3)$ & C71 & N18 & C73 & $110.0(3)$ \\
\hline C66 & $\mathrm{O} 3$ & C67 & $110.8(3)$ & C73 & N18 & $\mathrm{K} 1$ & $110.8(2)$ \\
\hline C67 & $\mathrm{O} 3$ & $\mathrm{~K} 1$ & $114.84(18)$ & C76 & 019 & $\mathrm{~K} 1$ & $112.9(2)$ \\
\hline C68 & O4 & $\mathrm{K} 1$ & $114.39(18)$ & C77 & 019 & $\mathrm{~K} 1$ & $117.4(2)$ \\
\hline C68 & $\mathrm{O} 4$ & C69 & $111.6(3)$ & C77 & O19 & C76 & $111.5(3)$ \\
\hline C69 & O4 & $\mathrm{K} 1$ & 118.63(18) & N3 & C6 & C7 & $111.0(3)$ \\
\hline C74 & O5 & K1 & $118.4(2)$ & C6 & C7 & C8 & $112.2(3)$ \\
\hline C74 & O5 & C75 & $112.1(3)$ & C7 & C8 & C9 & $110.1(3)$ \\
\hline C75 & O5 & $\mathrm{K} 1$ & $114.7(2)$ & C10 & C9 & C8 & $111.4(3)$ \\
\hline C81 & O6 & C79 & 111.1(3) & N3 & C10 & C9 & $112.5(3)$ \\
\hline P1 & N1 & $\mathrm{Ce} 1$ & $142.79(15)$ & N4 & C11 & C12 & $110.6(3)$ \\
\hline C6 & N3 & $\mathrm{P} 1$ & $120.2(2)$ & C11 & $\mathrm{C} 12$ & $\mathrm{C} 13$ & $110.2(3)$ \\
\hline C10 & N3 & P1 & 126.2(2) & C12 & C13 & C14 & $109.8(3)$ \\
\hline C10 & N3 & C6 & $111.8(3)$ & $\mathrm{C} 15$ & C14 & $\mathrm{C} 13$ & $109.4(3)$ \\
\hline C11 & N4 & P1 & $112.63(19)$ & N4 & C15 & C14 & $110.1(3)$ \\
\hline C15 & N4 & $\mathrm{P} 1$ & $120.9(2)$ & N6 & $\mathrm{C} 16$ & C17 & $110.9(3)$ \\
\hline C15 & N4 & C11 & 111.1(3) & C16 & C17 & C18 & $111.9(3)$ \\
\hline P2 & N5 & $\mathrm{Ce} 1$ & $160.1(2)$ & C17 & C18 & C19 & $109.8(3)$ \\
\hline C16 & N6 & P2 & $116.5(2)$ & C20 & C19 & C18 & $110.4(3)$ \\
\hline C16 & N6 & $\mathrm{C} 20$ & $110.5(3)$ & N6 & C20 & C19 & 112.1(3) \\
\hline C20 & N6 & P2 & 111.7(2) & N7 & C21 & C22 & $111.5(3)$ \\
\hline C21 & N7 & $\mathrm{P} 2$ & $120.1(2)$ & C23 & C22 & C21 & $111.4(4)$ \\
\hline C25 & N7 & P2 & $125.3(2)$ & C22 & C23 & C24 & $111.5(5)$ \\
\hline $\mathrm{C} 25$ & N7 & $\mathrm{C} 21$ & $114.3(3)$ & $\mathrm{C} 25$ & C24 & $\mathrm{C} 23$ & $112.0(4)$ \\
\hline C26 & N8 & P2 & $125.5(2)$ & N7 & C25 & C24 & $112.0(3)$ \\
\hline C30 & N8 & $\mathrm{P} 2$ & $119.0(2)$ & N8 & C26 & C27 & $112.2(3)$ \\
\hline C30 & N8 & $\mathrm{C} 26$ & $111.7(3)$ & C26 & $\mathrm{C} 27$ & $\mathrm{C} 28$ & $109.8(4)$ \\
\hline P3 & N9 & $\mathrm{Ce} 1$ & $147.42(15)$ & C29 & $\mathrm{C} 28$ & $\mathrm{C} 27$ & $110.7(4)$ \\
\hline C31 & N10 & P3 & $120.3(2)$ & C30 & $\mathrm{C} 29$ & $\mathrm{C} 28$ & $108.9(4)$ \\
\hline C35 & N10 & P3 & $125.0(2)$ & N8 & C30 & C29 & $111.1(3)$ \\
\hline C35 & N10 & C31 & $111.8(3)$ & N10 & C31 & C32 & $111.4(3)$ \\
\hline C36 & N11 & P3 & $115.0(2)$ & C33 & C32 & C31 & $110.7(3)$ \\
\hline C36 & N11 & C40 & $109.9(3)$ & C32 & C33 & C34 & $109.9(3)$ \\
\hline $\mathrm{C} 40$ & N11 & P3 & $112.8(2)$ & C35 & C34 & C33 & $110.0(3)$ \\
\hline C41 & N12 & P3 & $114.8(2)$ & N10 & C35 & C34 & $111.3(3)$ \\
\hline $\mathrm{C} 45$ & N12 & P3 & $122.2(2)$ & N11 & C36 & C37 & $109.4(3)$ \\
\hline C45 & N12 & C41 & $112.9(3)$ & C38 & C37 & C36 & $110.8(4)$ \\
\hline
\end{tabular}




\begin{tabular}{|c|c|c|c|}
\hline Atom & Atom & Atom & Angle $^{\circ}$ \\
\hline$\overline{\mathrm{C} 39}$ & C38 & C37 & $111.3(3)$ \\
\hline C40 & C39 & C38 & 111.3(3) \\
\hline N11 & C40 & C39 & $109.4(3)$ \\
\hline N12 & C41 & C42 & $110.2(3)$ \\
\hline C43 & C42 & C41 & $110.5(3)$ \\
\hline C44 & C43 & C42 & 109.7(3) \\
\hline C43 & C44 & C45 & $110.3(4)$ \\
\hline N12 & C45 & C44 & $110.4(3)$ \\
\hline N14 & C46 & C47 & $110.7(2)$ \\
\hline C46 & C47 & C48 & $110.0(3)$ \\
\hline C49 & C48 & C47 & 109.1(3) \\
\hline C50 & C49 & C48 & 111.1(3) \\
\hline N14 & C50 & C49 & $111.3(2)$ \\
\hline N16 & C56 & C57 & $110.4(3)$ \\
\hline C56 & C57 & C58 & $110.4(3)$ \\
\hline C59 & C58 & C57 & $110.0(3)$ \\
\hline C58 & C59 & C60 & $110.5(3)$ \\
\hline N16 & C60 & C59 & $110.6(3)$ \\
\hline $\mathrm{O} 1$ & C61 & C62 & 109.9(2) \\
\hline $\mathrm{O} 2$ & C62 & C61 & $108.0(2)$ \\
\hline $\mathrm{O} 2$ & C63 & C64 & $107.9(3)$ \\
\hline N17 & C64 & C63 & $113.5(3)$ \\
\hline N17 & C65 & C66 & 114.1(4) \\
\hline $\mathrm{O} 3$ & C66 & C65 & $108.4(3)$ \\
\hline O3 & C67 & C68 & 109.1(3) \\
\hline $\mathrm{O} 4$ & C68 & C67 & 109.5(3) \\
\hline $\mathrm{O} 4$ & C69 & C70 & $110.4(3)$ \\
\hline N18 & C70 & C69 & $113.6(3)$ \\
\hline N18 & C71 & $\mathrm{C} 72$ & $114.4(3)$ \\
\hline 01 & $\mathrm{C} 72$ & C71 & $108.8(3)$ \\
\hline C74 & $\mathrm{C} 73$ & N18 & $114.0(3)$ \\
\hline O5 & C74 & C73 & $109.2(3)$ \\
\hline O5 & $\mathrm{C} 75$ & C76 & $107.8(3)$ \\
\hline O19 & C76 & C75 & 109.1(3) \\
\hline O19 & C77 & C78 & 109.3(3) \\
\hline N17 & C78 & C77 & $115.2(3)$ \\
\hline O6 & C79 & C80 & 109.1(3) \\
\hline O6 & C81 & C82 & $108.9(3)$ \\
\hline C1_1 & N2_ 1 & P1 & $115.58(17)$ \\
\hline $\mathrm{C}^{-}{ }^{-1}$ & N2 1 & C5_1 & $110.1(2)$ \\
\hline $\mathrm{C} 5^{-1}$ & $\mathrm{~N} 2^{-} 1$ & $\mathrm{P} 1$ & $112.76(17)$ \\
\hline $\mathrm{N} 2-1$ & $\mathrm{C}^{-} 1$ & C2 1 & $110.4(2)$ \\
\hline $\mathrm{C} 1-1$ & $\mathrm{C} 2-1$ & $\mathrm{C} 3-1$ & $112.0(3)$ \\
\hline C4_1 & C3-1 & $\mathrm{C} 2-1$ & $110.3(2)$ \\
\hline $\mathrm{C} 3^{-1}$ & $\mathrm{C}^{-} 1$ & $\mathrm{C} 5^{-} 1$ & 111.2(2) \\
\hline $\mathrm{N} 2-1$ & $\mathrm{C} 5^{-} 1$ & $\mathrm{C}^{-} 1$ & $109.4(2)$ \\
\hline $\mathrm{C} 1-3$ & $\mathrm{~N} 2{ }^{-} 3$ & $\mathrm{P} 4$ & $124.1(3)$ \\
\hline $\mathrm{C} 53$ & N2 3 & P4 & $117.5(3)$ \\
\hline $\mathrm{C} 53$ & $\mathrm{~N} 2{ }^{-} 3$ & C1 3 & $110.9(3)$ \\
\hline $\mathrm{N} 2-3$ & $\mathrm{C}^{-} 3$ & $\mathrm{C} 2-3$ & $110.8(3)$ \\
\hline $\mathrm{C} 13$ & $\mathrm{C} 23$ & $\mathrm{C} 33$ & 111.7(3) \\
\hline $\mathrm{C} 4-3$ & $\mathrm{C} 33$ & $\mathrm{C} 23$ & $109.8(3)$ \\
\hline $\mathrm{C} 3{ }^{-} 3$ & $\mathrm{C}^{-} 3$ & $\mathrm{C} 5^{-} 3$ & $111.3(3)$ \\
\hline $\mathrm{N} 2-3$ & $\mathrm{C} 5{ }^{-} 3$ & $\mathrm{C} 4-3$ & 109.6(3) \\
\hline
\end{tabular}

\begin{tabular}{lllr}
\hline Atom & Atom & Atom & Angle $^{\circ}$ \\
\hline C1_2 & N2_2 & P4 & $117.3(3)$ \\
C5_2 & N2_2 & P4 & $120.1(3)$ \\
C5_2 & N2_2 & C1_2 & $110.9(3)$ \\
N2_2 & C1_2 & C2_2 & $110.3(3)$ \\
C1_2 & C2_2 & C3_2 & $111.7(3)$ \\
C4_2 & C3_2 & C2_2 & $110.1(3)$ \\
C3_2 & C4_2 & C5_2 & $111.6(3)$ \\
N2_2 & C5_2 & C4_2 & $110.0(3)$
\end{tabular}




\section{Electronic Absorption Spectra}

All UV-vis measurements were performed in THF.

A

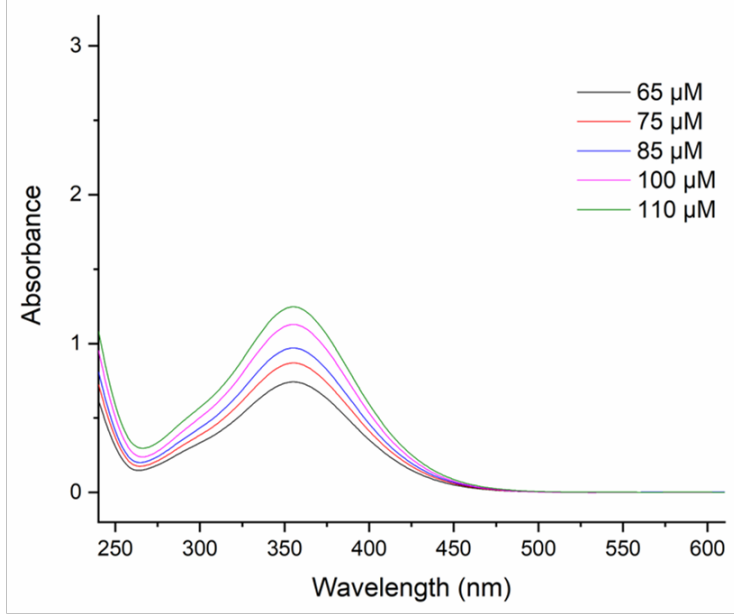

B

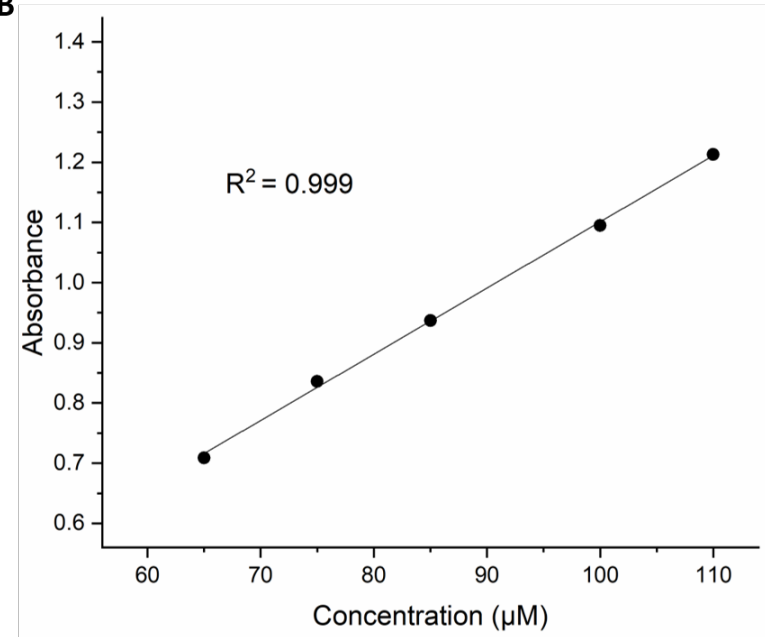

Figure S20. A) UV-vis spectra of 2-Ce. B) Linear regression of absorbance at $355 \mathrm{~nm}$ maximum where $\varepsilon=11,000 \mathrm{~cm}^{-1} \mathrm{M}^{-1}$.

A

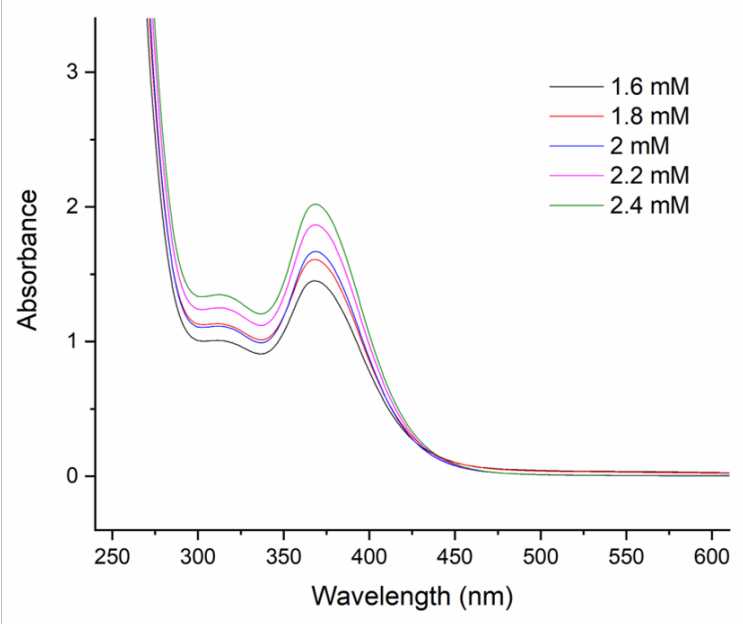

B

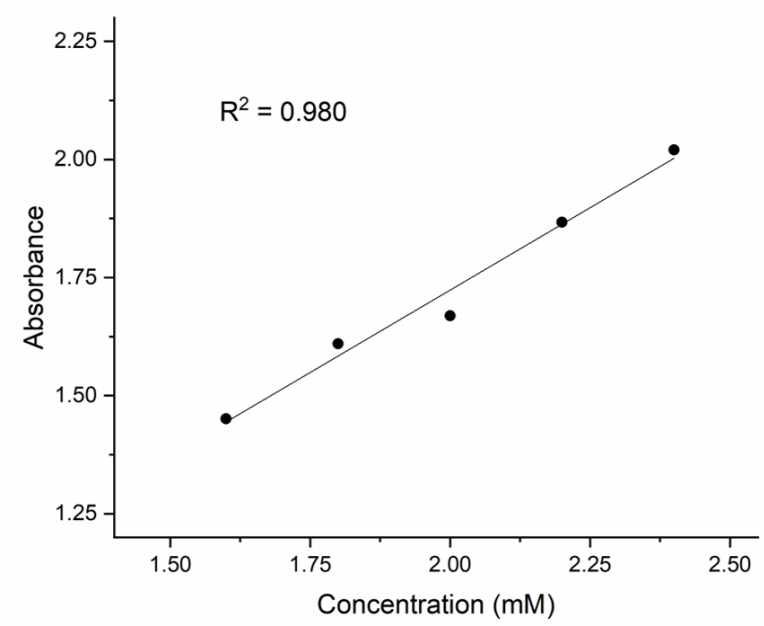

Figure S21. A) UV-vis spectra of 3-Ce-K(Et $\left.{ }_{2} \mathrm{O}\right)$. B) Linear regression of absorbance at $366 \mathrm{~nm}$ maximum where $\varepsilon=600 \mathrm{~cm}^{-1} \mathrm{M}^{-1}$. 

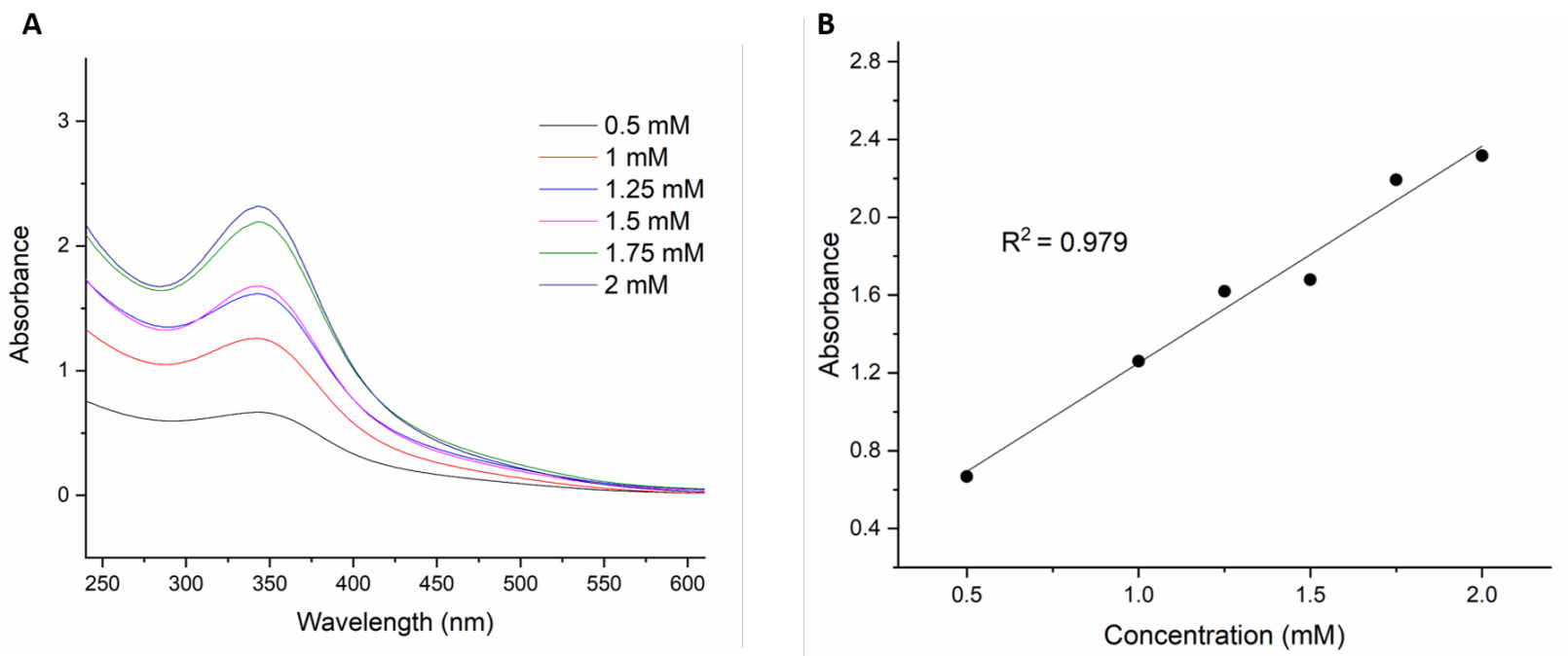

Figure S22. A) UV-vis spectra of 3-Ce-K222. B) Linear regression of absorbance at 361 $\mathrm{nm}$ maximum where $\varepsilon=1115 \mathrm{~cm}^{-1} \mathrm{M}^{-1}$.

\section{Magnetism}

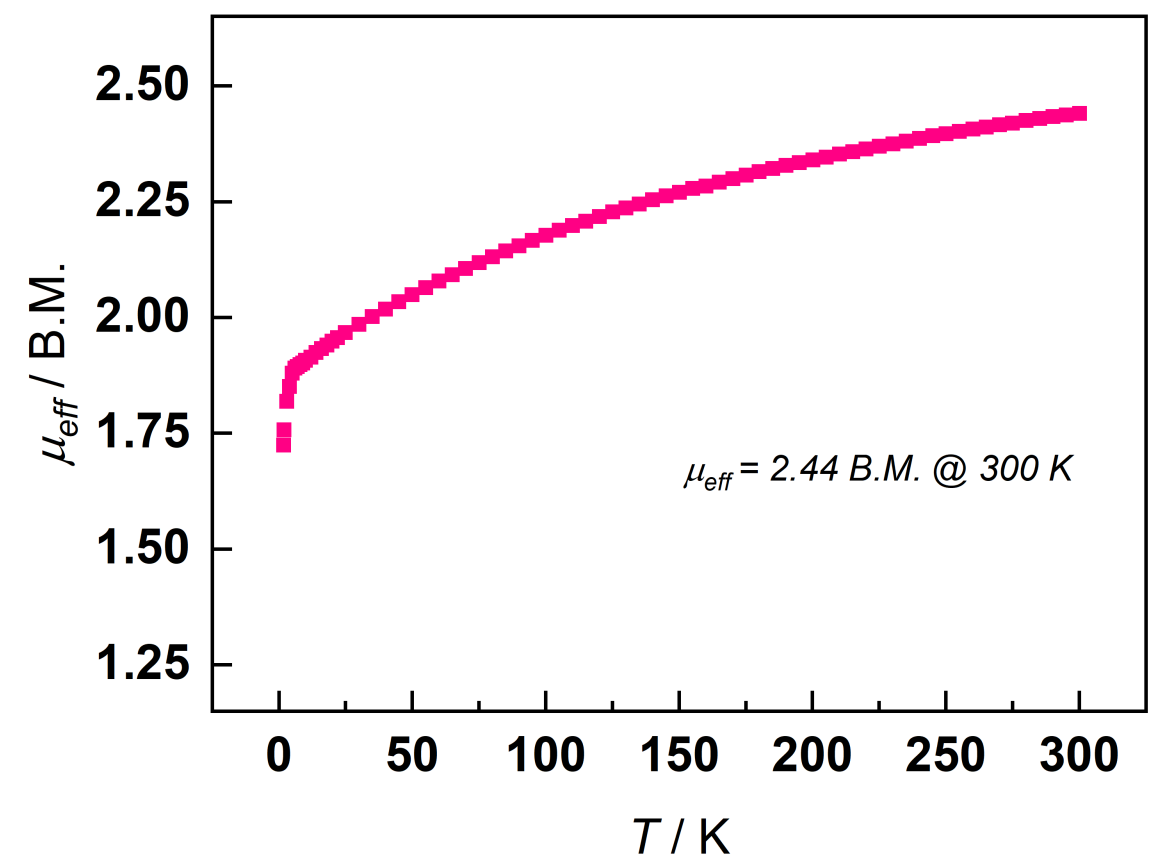

Figure S23. Effective magnetic moment $\left(U_{\text {eff }}\right)$ vs. T plot for $3-\mathrm{Ce}-\mathrm{K}\left(\mathrm{Et}_{2} \mathrm{O}\right)$. 


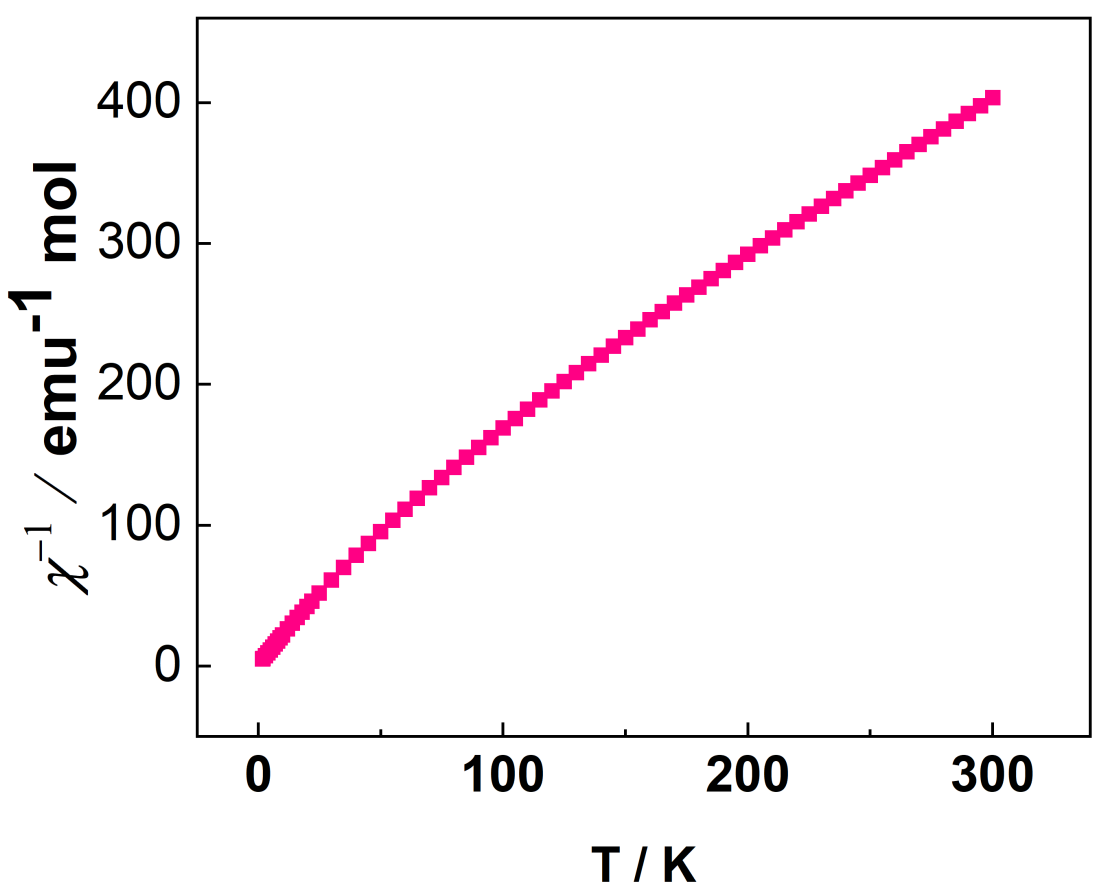

Figure S24. $1 / \chi$ vs. T plot for $3-\mathrm{Ce}-\mathrm{K}\left(\mathrm{Et}_{2} \mathrm{O}\right)$. 


\section{Theoretical Calculations}

$\mathrm{Ce}(4+)$

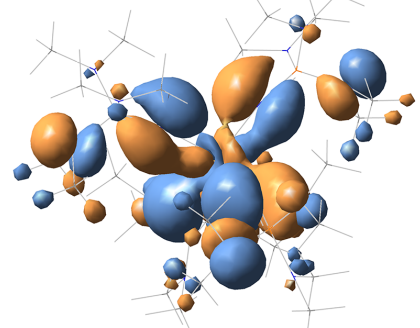

HOMO ( $\pi$ bonding)

(5.1\%4f)

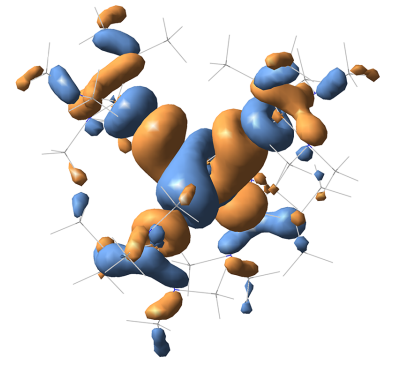

HOMO-20 ( $\sigma$ bonding) $(12.1 \% 5 d)$

$\mathrm{Ce}(3+)$

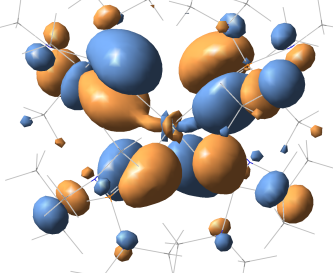

HOMO (non-bonding)

$(0.7 \% 4 f)$

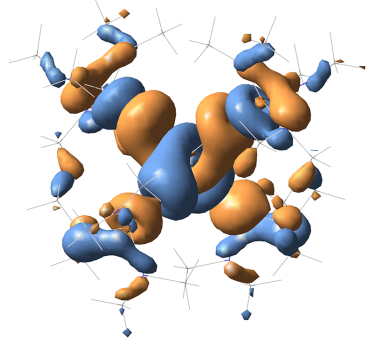

HOMO-20 ( $\sigma$ bonding) $(7.8 \% 5 \mathrm{~d})$

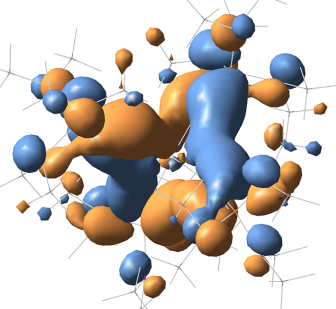

HOMO-10 ( $\pi$ bonding)

$(5.4 \% 5 d)$

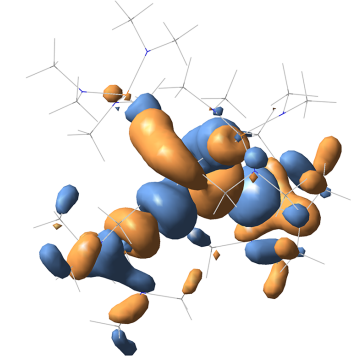

HOMO-21 ( $\sigma$ bonding) $(12.2 \% 5 \mathrm{~d})$

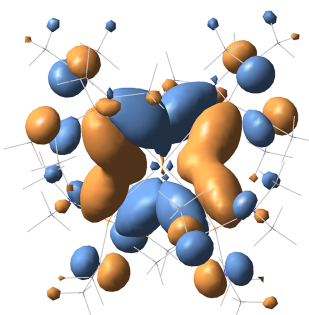

HOMO-6 ( $\pi$ bonding) $(6.2 \% 5 d)$

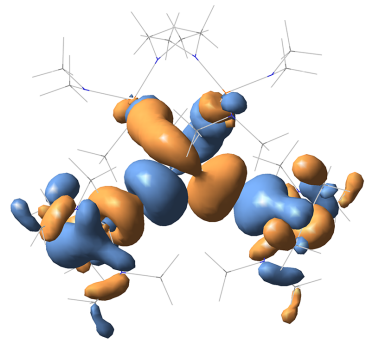

HOMO-21 ( $\sigma$ bonding) $(7.8 \% 5 \mathrm{~d})$

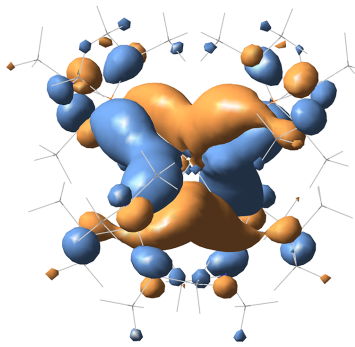

HOMO-11 ( $\pi$ bonding)

$(7.4 \% 5 d)$

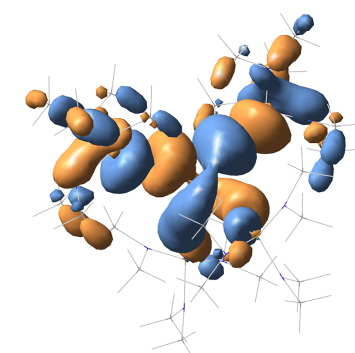

HOMO-22 ( $\sigma$ bonding) $(12.3 \% 5 d)$

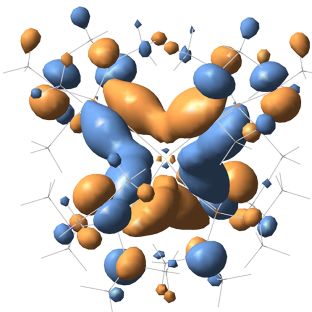

HOMO-7 ( $\pi$ bonding) $(5.0 \% 5 \mathrm{~d})$

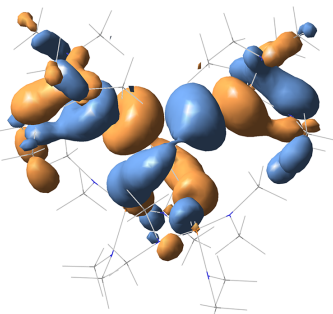

HOMO-22 ( $\sigma$ bonding)

$(8.5 \% 5 d)$

Figure S25. Metal atomic valence orbital compositions and bonding character of some typical molecular orbitals in $\left[\mathrm{Ce}\left(\mathrm{NP}\left(\mathrm{NMe}_{2}\right)_{3}\right)_{4}\right]^{0 /-}$. $\mathrm{HOMO}$ is labelled for the first ligand 
based occupied orbital. For the $\mathrm{Ce}\left(3^{+}\right)$compound, the compositions are averaged for alpha and beta orbitals.

Table S12. Experimental (Expt.) and Optimized (Opt.) Average M-N and M-O Bond Lengths for $\left[\mathrm{Ce}\left(\mathrm{NP}\left(\mathrm{NMe}_{2}\right)_{3}\right)_{4}\right]^{0 /-}$ and $\left[\mathrm{K}(\mathrm{DME})_{2}\right]\left[\mathrm{Ce}\left(\mathrm{NP}\left(\mathrm{NMe}_{2}\right)_{3}\right)_{4}\right]^{+/ 0}$.

$\begin{array}{lllll}\text { Compound } & & \mathrm{Ce}-\mathrm{N}^{a} & \mathrm{~K}^{-\mathrm{N}^{a}} & \mathrm{~K}-\mathrm{O}^{a} \\ \left.\mathrm{Ce}\left[\mathrm{NP}\left(\mathrm{NMe}_{2}\right)_{3}\right)_{4}\right] & \text { Opt. } & 2.20 & & \\ \text { 2-Ce } & \text { Expt. } & 2.20 & & \\ \left.\mathrm{Ce}\left[\mathrm{NP}\left(\mathrm{NMe}_{2}\right)_{3}\right)_{4}\right]^{-} & \text {Opt. } & 2.34 & & \\ 3-\mathrm{Ce}-\mathrm{K2222} & \text { Expt. } & 2.32 & & \\ {\left[\mathrm{~K}(\mathrm{DME})_{2}\right]\left[\mathrm{Ce}\left(\mathrm{NP}\left(\mathrm{NMe}_{2}\right)_{3}\right)_{4}\right]^{+}} & \text {Opt. } & 2.21 & 3.05 & 2.95 \\ {\left[\mathrm{~K}(\mathrm{DME})_{2}\right]\left[\mathrm{Ce}\left(\mathrm{NP}\left(\mathrm{NMe}_{2}\right)_{3}\right)_{4}\right]} & \text { Opt. } & 2.36 & 2.87 & 2.92 \\ \text { 3-Ce-K(DME})_{2} & \text { Expt. } & 2.33 & 2.79 & 2.95\end{array}$

ain Angstroms.

Table S13. Gibbs free energies in $\mathrm{kcal} / \mathrm{mol}$ of ligand replacement reactions.

\begin{tabular}{c|c} 
Reactions & $\Delta G$ \\
{$\left[\mathrm{Ce}\left(\mathrm{pyNO}_{4}\right)_{+}+4 \mathrm{NP}\left(\mathrm{NMe}_{2}\right)_{3}{ }^{-} \rightarrow\left[\mathrm{Ce}\left(\mathrm{NP}\left(\mathrm{NMe}_{2}\right)_{3}\right)_{4}\right]+4 \mathrm{PyNO}^{-}\right.$} & 1.3 \\
{$\left[\mathrm{Ce}\left(\mathrm{pyNO}_{4}\right]^{-}+4 \mathrm{NP}\left(\mathrm{NMe}_{2}\right)_{3}{ }^{-} \rightarrow\left[\mathrm{Ce}\left(\mathrm{NP}\left(\mathrm{NMe}_{2}\right)_{3}\right)_{4}\right]^{-}+4 \mathrm{PyNO}^{-}\right.$} & 9.1
\end{tabular}

Table S14. Optimized Cartesian coordinates of $\mathrm{Ce}(3+/ 4+)$ complexes with $\left[\mathrm{NP}\left(\mathrm{NMe}_{2}\right)_{3}\right]^{-}$.

\begin{tabular}{|lrrr|lrrr|}
\hline \multicolumn{2}{|l|}{$\left.\mathrm{Ce}\left(\mathrm{NP}\left(\mathrm{NMe}_{2}\right)_{3}\right)_{4}\right]$} & & \multicolumn{4}{l|}{$\left[\mathrm{Ce}\left(\mathrm{NP}(\mathrm{NMe})_{3}\right) 4\right]^{-}$} \\
$\mathrm{Ce}$ & 0.00824900 & -0.00551100 & 0.04421800 & $\mathrm{Ce}$ & -0.00061200 & 0.00153500 & 0.03098400 \\
$\mathrm{~N}$ & -1.52108400 & 0.48753900 & 1.54474700 & $\mathrm{~N}$ & -1.61000600 & 0.99240900 & 1.41426400 \\
$\mathrm{~N}$ & 0.93629600 & 1.83395700 & -0.71720200 & $\mathrm{~N}$ & 0.99500700 & 1.63360100 & -1.32589500 \\
$\mathrm{P}$ & -2.61502800 & 1.07634200 & 2.47408400 & $\mathrm{P}$ & -2.62093700 & 1.89559500 & 2.12361100 \\
$\mathrm{P}$ & 1.91849600 & 2.89291800 & -1.28523000 & $\mathrm{P}$ & 2.01402600 & 2.40179500 & -2.17081200 \\
$\mathrm{~N}$ & -3.42602500 & 2.52601200 & 2.05036000 & $\mathrm{~N}$ & -3.38696000 & 3.22792500 & 1.31330600 \\
$\mathrm{C}$ & -4.33061100 & 2.47343700 & 0.90159100 & $\mathrm{C}$ & -4.31016800 & 2.87073700 & 0.23789300 \\
$\mathrm{H}$ & -4.91414100 & 1.55568600 & 0.92144900 & $\mathrm{H}$ & -4.96057300 & 2.05895900 & 0.55845000 \\
$\mathrm{H}$ & -3.78978500 & 2.51874700 & -0.05478600 & $\mathrm{H}$ & -3.78666500 & 2.55018000 & -0.67534500 \\
$\mathrm{C}$ & -2.61913700 & 3.74780000 & 2.03076700 & $\mathrm{C}$ & -2.47926800 & 4.29078800 & 0.88034000 \\
$\mathrm{H}$ & -2.02485400 & 3.83537800 & 1.11093000 & $\mathrm{H}$ & -1.90076300 & 4.00915100 & -0.01094900 \\
$\mathrm{H}$ & -1.93920700 & 3.76563900 & 2.88001700 & $\mathrm{H}$ & -1.77676600 & 4.53116500 & 1.67680900 \\
$\mathrm{~N}$ & -1.99488900 & 1.52771300 & 3.99914400 & $\mathrm{~N}$ & -1.96305100 & 2.76704300 & 3.46079300 \\
$\mathrm{C}$ & -0.78647700 & 0.88399900 & 4.49550800 & $\mathrm{C}$ & -0.77316800 & 2.24357700 & 4.10794200 \\
$\mathrm{H}$ & -0.22383500 & 0.46884400 & 3.66259500 & $\mathrm{H}$ & -0.27502300 & 1.54635700 & 3.43707400 \\
$\mathrm{H}$ & -1.01069100 & 0.07563300 & 5.20795200 & $\mathrm{H}$ & -1.00571900 & 1.72086000 & 5.05084200 \\
$\mathrm{C}$ & -2.79116200 & 2.15288800 & 5.04502200 & $\mathrm{C}$ & -2.67466900 & 3.75031500 & 4.25184100 \\
$\mathrm{H}$ & -3.17930400 & 1.42498800 & 5.77269800 & $\mathrm{H}$ & -3.08236900 & 3.33594000 & 5.18916500 \\
$\mathrm{H}$ & -3.63035900 & 2.69598200 & 4.61488600 & $\mathrm{H}$ & -3.49445800 & 4.17872000 & 3.67756400 \\
\hline
\end{tabular}




\begin{tabular}{|c|c|c|c|c|c|c|c|}
\hline $\mathrm{N}$ & -3.95765700 & 0.02955200 & 2.62943000 & $\mathrm{~N}$ & -4.04995700 & 1.06455400 & 2.63377600 \\
\hline C & -5.09904900 & 0.33277600 & 3.48119000 & C & -5.10404000 & 1.68957000 & 3.40829500 \\
\hline $\mathrm{H}$ & -5.25858600 & 1.40795300 & 3.54001400 & $\mathrm{H}$ & -5.20087100 & 2.74093400 & 3.14020300 \\
\hline $\mathrm{H}$ & -4.98388900 & -0.06126500 & 4.50202000 & $\mathrm{H}$ & -4.94199000 & 1.62053700 & 4.49751900 \\
\hline C & -3.76484400 & -1.39782400 & 2.41014300 & C & -3.95816400 & -0.37177200 & 2.83522300 \\
\hline $\mathrm{H}$ & -3.56512000 & -1.94148900 & 3.34641100 & $\mathrm{H}$ & -3.75156300 & -0.63886200 & 3.88571400 \\
\hline $\mathrm{H}$ & -2.93018900 & -1.55205400 & 1.73070800 & $\mathrm{H}$ & -3.15883700 & -0.76820900 & 2.21260200 \\
\hline $\mathrm{N}$ & 1.36507500 & 3.55964900 & -2.75618300 & $\mathrm{~N}$ & 1.51821600 & 2.63115900 & -3.81119000 \\
\hline C & 2.09097400 & 4.58295100 & -3.49370400 & C & 2.27945800 & 3.38212200 & -4.78913800 \\
\hline $\mathrm{H}$ & 3.16069800 & 4.50154800 & -3.31000100 & $\mathrm{H}$ & 3.34602300 & 3.30317900 & -4.58281000 \\
\hline $\mathrm{H}$ & 1.76663400 & 5.60207800 & -3.23550200 & $\mathrm{H}$ & 2.00820800 & 4.45118200 & -4.82246700 \\
\hline C & -0.05819700 & 3.52099700 & -3.06153000 & C & 0.09693100 & 2.56706700 & -4.10391700 \\
\hline $\mathrm{H}$ & -0.56604700 & 4.46216500 & -2.80024700 & $\mathrm{H}$ & -0.37323500 & 3.56518800 & -4.13020400 \\
\hline $\mathrm{H}$ & -0.52428800 & 2.70586700 & -2.51323700 & $\mathrm{H}$ & -0.39631600 & 1.96938400 & -3.34010600 \\
\hline $\mathrm{N}$ & 2.28406800 & 4.11049800 & -0.14592800 & $\mathrm{~N}$ & 2.46561100 & 3.92648400 & -1.49568600 \\
\hline C & 3.19073600 & 5.21705500 & -0.41352800 & C & 3.43969400 & 4.83389400 & -2.06695200 \\
\hline $\mathrm{H}$ & 3.93419300 & 4.93274400 & -1.15580400 & $\mathrm{H}$ & 4.15797100 & 4.28643700 & -2.67521600 \\
\hline $\mathrm{H}$ & 3.72264100 & 5.48789000 & 0.50677000 & $\mathrm{H}$ & 3.99688200 & 5.34000000 & -1.26496900 \\
\hline C & 1.34703100 & 4.37499800 & 0.93655400 & C & 1.55477500 & 4.55840100 & -0.55855200 \\
\hline $\mathrm{H}$ & 1.89713800 & 4.59424600 & 1.86003100 & $\mathrm{H}$ & 2.11679100 & 5.01590600 & 0.26815200 \\
\hline $\mathrm{H}$ & 0.72256600 & 3.49996100 & 1.10033300 & $\mathrm{H}$ & 0.88207000 & 3.80575800 & -0.15186500 \\
\hline $\mathrm{N}$ & 3.50561300 & 2.44175500 & -1.74810900 & $\mathrm{~N}$ & 3.60842100 & 1.78665700 & -2.47706400 \\
\hline C & 4.41368700 & 2.02991700 & -0.67498700 & C & 4.45932100 & 1.65110300 & -1.29349200 \\
\hline $\mathrm{H}$ & 4.21893400 & 1.00206600 & -0.33963700 & $\mathrm{H}$ & 4.17683600 & 0.79035100 & -0.67122200 \\
\hline $\mathrm{H}$ & 4.31100500 & 2.69314300 & 0.18128100 & $\mathrm{H}$ & 4.39147600 & 2.54807200 & -0.67999900 \\
\hline C & 3.62835900 & 1.56175300 & -2.91095000 & C & 3.67101500 & 0.57779700 & -3.29704400 \\
\hline $\mathrm{H}$ & 2.92957800 & 1.86349500 & -3.68830900 & $\mathrm{H}$ & 3.00227300 & 0.67079300 & -4.15093600 \\
\hline $\mathrm{H}$ & 3.43039800 & 0.51025400 & -2.65738600 & $\mathrm{H}$ & 3.38666100 & -0.32464200 & -2.73559600 \\
\hline $\mathrm{N}$ & 1.52588300 & -1.24660900 & 1.03731500 & $\mathrm{~N}$ & 1.60102800 & -0.96075200 & 1.44335400 \\
\hline $\mathrm{N}$ & -0.89981600 & -1.11643900 & -1.62043600 & $\mathrm{~N}$ & -0.98942000 & -1.65910700 & -1.29578100 \\
\hline $\mathrm{P}$ & 2.56825800 & -2.29309300 & 1.51198200 & $\mathrm{P}$ & 2.60755600 & -1.85095200 & 2.17508100 \\
\hline $\mathrm{P}$ & -1.86570500 & -1.67124700 & -2.70121700 & $\mathrm{P}$ & -2.00176100 & -2.44663800 & -2.13084000 \\
\hline $\mathrm{N}$ & 3.38422600 & -3.27942200 & 0.37182700 & $\mathrm{~N}$ & 3.37860000 & -3.19815200 & 1.39469100 \\
\hline C & 4.34077500 & -2.61832400 & -0.51626900 & C & 4.30706000 & -2.86080100 & 0.31736100 \\
\hline $\mathrm{H}$ & 4.93000000 & -1.89078200 & 0.03751200 & $\mathrm{H}$ & 4.95514200 & -2.04237000 & 0.62548100 \\
\hline $\mathrm{H}$ & 3.84363400 & -2.09911500 & -1.34836700 & $\mathrm{H}$ & 3.78808200 & -2.55845500 & -0.60460600 \\
\hline C & 2.56602400 & -4.24784500 & -0.36097100 & C & 2.47404800 & -4.27000200 & 0.97742300 \\
\hline $\mathrm{H}$ & 2.01114100 & -3.77949700 & -1.18548000 & $\mathrm{H}$ & 1.89856400 & -4.00538500 & 0.07901900 \\
\hline $\mathrm{H}$ & 1.84972000 & -4.72031700 & 0.30805300 & $\mathrm{H}$ & 1.76897800 & -4.49710400 & 1.77550100 \\
\hline $\mathrm{N}$ & 1.87499000 & -3.50409100 & 2.49584200 & $\mathrm{~N}$ & 1.94114300 & -2.69784700 & 3.52390400 \\
\hline C & 0.66006000 & -3.20471500 & 3.24137500 & C & 0.74975700 & -2.15934400 & 4.15603800 \\
\hline $\mathrm{H}$ & 0.13677000 & -2.37420700 & 2.77333600 & $\mathrm{H}$ & 0.25385400 & -1.47693800 & 3.46851000 \\
\hline $\mathrm{H}$ & 0.87116700 & -2.94242200 & 4.28925600 & $\mathrm{H}$ & 0.98001500 & -1.61595500 & 5.08779700 \\
\hline C & 2.61993700 & -4.63386600 & 3.03234800 & C & 2.65116200 & -3.66130400 & 4.34046700 \\
\hline $\mathrm{H}$ & 2.99052600 & -4.45003800 & 4.05165600 & $\mathrm{H}$ & 3.05495700 & -3.22446800 & 5.26927800 \\
\hline $\mathrm{H}$ & 3.46675200 & -4.87305000 & 2.39209200 & $\mathrm{H}$ & 3.47355600 & -4.10204000 & 3.77938600 \\
\hline $\mathrm{N}$ & 3.90982700 & -1.55960600 & 2.27497400 & $\mathrm{~N}$ & 4.03333300 & -1.01037100 & 2.67852900 \\
\hline C & 5.01055800 & -2.32310800 & 2.84497100 & C & 5.08696400 & -1.62073900 & 3.46508400 \\
\hline $\mathrm{H}$ & 5.15396900 & -3.25450700 & 2.30011800 & $\mathrm{H}$ & 5.18231400 & -2.67746700 & 3.21860700 \\
\hline $\mathrm{H}$ & 4.85794300 & -2.55879100 & 3.90878300 & $\mathrm{H}$ & 4.92538200 & -1.52909100 & 4.55271900 \\
\hline C & 3.74693000 & -0.24251100 & 2.87573900 & C & 3.94132600 & 0.42943100 & 2.85251100 \\
\hline $\mathrm{H}$ & 3.52025200 & -0.29941800 & 3.95150600 & $\mathrm{H}$ & 3.73415000 & 0.71616100 & 3.89769800 \\
\hline $\mathrm{H}$ & 2.94047600 & 0.28797400 & 2.37533500 & $\mathrm{H}$ & 3.14226900 & 0.81375700 & 2.22197700 \\
\hline $\mathrm{N}$ & -1.25557200 & -1.44963100 & -4.28040200 & $\mathrm{~N}$ & -1.49532400 & -2.70901700 & -3.76315200 \\
\hline C & -1.95634300 & -1.88767400 & -5.47826400 & C & -2.24952400 & -3.48107400 & -4.73011300 \\
\hline $\mathrm{H}$ & -3.03233000 & -1.88820000 & -5.31419200 & $\mathrm{H}$ & -3.31738000 & -3.39998000 & -4.53156000 \\
\hline
\end{tabular}




\begin{tabular}{|c|c|c|c|c|c|c|c|}
\hline $\mathrm{H}$ & -1.65028800 & -2.89264100 & -5.80511000 & $\mathrm{H}$ & -1.97608400 & -4.55006300 & -4.74022600 \\
\hline C & 0.17916300 & -1.28905100 & -4.47127300 & C & -0.07217300 & -2.65004400 & -4.04782600 \\
\hline $\mathrm{H}$ & 0.67126700 & -2.23472900 & -4.74606800 & $\mathrm{H}$ & 0.39920200 & -3.64795000 & -4.04874300 \\
\hline $\mathrm{H}$ & 0.62695000 & -0.91324500 & -3.55453800 & $\mathrm{H}$ & 0.41548700 & -2.03476300 & -3.29446800 \\
\hline $\mathrm{N}$ & -2.28579100 & -3.30170600 & -2.41688900 & $\mathrm{~N}$ & -2.45515800 & -3.95760200 & -1.42698000 \\
\hline C & -3.18266300 & -4.06170900 & -3.27494500 & C & -3.42360900 & -4.87884400 & -1.98557600 \\
\hline $\mathrm{H}$ & -3.89417700 & -3.40102900 & -3.76665000 & $\mathrm{H}$ & -4.13774000 & -4.34614300 & -2.61153500 \\
\hline $\mathrm{H}$ & -3.75263300 & -4.77728900 & -2.66975600 & $\mathrm{H}$ & -3.98647400 & -5.36784600 & -1.17696400 \\
\hline C & -1.39363700 & -4.13642500 & -1.62480100 & C & -1.55072400 & -4.56735000 & -0.46922400 \\
\hline $\mathrm{H}$ & -1.98122300 & -4.81154800 & -0.99046000 & $\mathrm{H}$ & -2.11825400 & -5.00602400 & 0.36391900 \\
\hline $\mathrm{H}$ & -0.77498800 & -3.50791500 & -0.98861700 & $\mathrm{H}$ & -0.88118100 & -3.80519500 & -0.07525500 \\
\hline $\mathrm{N}$ & -3.42991300 & -0.99906400 & -2.89619000 & $\mathrm{~N}$ & -3.59526000 & -1.84078500 & -2.46032000 \\
\hline C & -4.38386400 & -1.21356100 & -1.80565600 & C & -4.45440500 & -1.68270800 & -1.28546400 \\
\hline $\mathrm{H}$ & -4.20642900 & -0.53461300 & -0.96043300 & $\mathrm{H}$ & -4.17700400 & -0.80948000 & -0.67846800 \\
\hline $\mathrm{H}$ & -4.31606100 & -2.23637700 & -1.44127000 & $\mathrm{H}$ & -4.39002500 & -2.56727500 & -0.65386100 \\
\hline C & -3.50124100 & 0.37319800 & -3.39931700 & C & -3.65373200 & -0.64838700 & -3.30450500 \\
\hline $\mathrm{H}$ & -2.77314600 & 0.52252700 & -4.19376800 & $\mathrm{H}$ & -2.97905300 & -0.75739700 & -4.15180600 \\
\hline $\mathrm{H}$ & -3.30883700 & 1.11432900 & -2.61014900 & $\mathrm{H}$ & -3.37430900 & 0.26533400 & -2.75909100 \\
\hline $\mathrm{H}$ & 1.97037200 & -5.51686000 & 3.07421900 & $\mathrm{H}$ & 1.97297000 & -4.47506700 & 4.63685200 \\
\hline $\mathrm{H}$ & -0.00067000 & -4.08033500 & 3.24353100 & $\mathrm{H}$ & 0.05425700 & -2.97172400 & 4.41023900 \\
\hline $\mathrm{H}$ & 5.93842500 & -1.74373400 & 2.76754400 & $\mathrm{H}$ & 6.04779600 & -1.13682200 & 3.24028800 \\
\hline $\mathrm{H}$ & 5.01738200 & -3.36738900 & -0.94061600 & $\mathrm{H}$ & 4.92829100 & -3.73451200 & 0.08496800 \\
\hline $\mathrm{H}$ & 3.21416300 & -5.02352500 & -0.78193000 & $\mathrm{H}$ & 3.05640400 & -5.17321100 & 0.75787500 \\
\hline $\mathrm{H}$ & 4.64608200 & 1.62793600 & -3.30956300 & $\mathrm{H}$ & 4.69528100 & 0.43889100 & -3.66437900 \\
\hline $\mathrm{H}$ & 5.44587700 & 2.08380600 & -1.03650800 & $\mathrm{H}$ & 5.50175800 & 1.52058800 & -1.60880000 \\
\hline $\mathrm{H}$ & 1.92488700 & 4.44879500 & -4.56954100 & $\mathrm{H}$ & 2.10204700 & 2.97348900 & -5.79403200 \\
\hline $\mathrm{H}$ & -0.20234400 & 3.35062600 & -4.13537600 & $\mathrm{H}$ & -0.06535200 & 2.09783100 & -5.08406400 \\
\hline $\mathrm{H}$ & 2.66713300 & 6.11751200 & -0.76755500 & $\mathrm{H}$ & 2.98294500 & 5.62218300 & -2.68900800 \\
\hline $\mathrm{H}$ & 0.69744400 & 5.23731400 & 0.72158900 & $\mathrm{H}$ & 0.95259700 & 5.35352700 & -1.03010800 \\
\hline $\mathrm{H}$ & -2.17404200 & 2.87161800 & 5.59829900 & $\mathrm{H}$ & -1.99654100 & 4.57009100 & 4.53150900 \\
\hline $\mathrm{H}$ & -0.15814200 & 1.61953600 & 5.01284700 & $\mathrm{H}$ & -0.07867500 & 3.06175200 & 7800 \\
\hline $\mathrm{H}$ & -5.01663300 & 3.32588800 & 0.94272100 & $\mathrm{H}$ & -4.92931300 & 3.74036000 & -0.01455900 \\
\hline $\mathrm{H}$ & -3.28001300 & 4.61851200 & 2.09491500 & $\mathrm{H}$ & -3.05924900 & 5.19088000 & 0.64237500 \\
\hline $\mathrm{H}$ & -6.00402600 & -0.11756000 & 3.05617700 & $\mathrm{H}$ & -6.06414800 & 1.19985300 & 3.19364400 \\
\hline $\mathrm{H}$ & -4.66841800 & -1.82736800 & 1.96139400 & $\mathrm{H}$ & -4.90452300 & -0.85211400 & 2.55109500 \\
\hline $\mathrm{H}$ & -2.64637300 & -4.63611500 & -4.04483500 & $\mathrm{H}$ & -2.96085500 & -5.68008400 & -2.58628500 \\
\hline $\mathrm{H}$ & -0.73705200 & -4.75620800 & -2.25454200 & $\mathrm{H}$ & -0.94508500 & -5.37280100 & -0.91823900 \\
\hline $\mathrm{H}$ & -4.50226800 & 0.55981200 & -3.80203300 & $\mathrm{H}$ & -4.67563600 & -0.51824000 & -3.68146800 \\
\hline $\mathrm{H}$ & -5.39963800 & -1.04157500 & -2.17680600 & $\mathrm{H}$ & -5.49470500 & -1.55948500 & -1.61053500 \\
\hline $\mathrm{H}$ & 4.67299400 & 0.33340000 & 2.76001700 & $\mathrm{H}$ & 4.88774200 & 0.90464300 & 2.55993600 \\
\hline $\mathrm{H}$ & 0.36739000 & -0.56839000 & -5.27637600 & $\mathrm{H}$ & 0.09601100 & -2.20270400 & -5.03716000 \\
\hline $\mathrm{H}$ & -1.74390600 & -1.19608200 & -6.30265200 & $\mathrm{H}$ & -2.06703900 & -3.09251800 & -5.74205800 \\
\hline \multicolumn{4}{|c|}{$\left[\mathrm{K}(\mathrm{DME})_{2}\right]\left[\mathrm{Ce}\left(\mathrm{NP}\left(\mathrm{NMe}_{2}\right)_{3}\right)_{4}\right]^{+}$} & \multicolumn{4}{|c|}{$\left[\mathrm{K}(\mathrm{DME})_{2]}\left[\mathrm{Ce}\left(\mathrm{NP}\left(\mathrm{NMe}_{2}\right)_{3}\right)_{4]}\right.\right.$} \\
\hline $\mathrm{Ce}$ & 0.93913400 & 0.00004600 & -0.00000800 & $\mathrm{Ce}$ & -0.71827300 & -0.00474400 & -0.00147000 \\
\hline $\mathrm{N}$ & 2.21294500 & 1.22656400 & -1.25604500 & $\mathrm{~N}$ & -2.24212800 & -1.50083900 & -0.89106300 \\
\hline $\mathrm{N}$ & -0.46920400 & -1.23343100 & -1.23648100 & $\mathrm{~N}$ & 0.87406600 & 0.66849600 & -1.67187600 \\
\hline $\mathrm{K}$ & -2.97254300 & -0.00007100 & -0.00006300 & $\mathrm{~K}$ & 3.04497700 & -0.15517600 & -0.03580100 \\
\hline $\mathrm{P}$ & 3.01856200 & 2.05440700 & -2.30859400 & $\mathrm{P}$ & -3.23891900 & -2.40179200 & -1.64301900 \\
\hline $\mathrm{P}$ & -0.63642000 & -2.55886200 & -2.04592400 & $\mathrm{P}$ & 1.06004300 & 1.75829700 & -2.75129700 \\
\hline 0 & -5.14579000 & 1.76608300 & 0.88886200 & $\mathrm{O}$ & 5.34812700 & -2.04706900 & 0.16551300 \\
\hline 0 & -4.44539400 & 1.78103800 & -1.85532500 & $\mathrm{O}$ & 3.28056500 & -2.41523700 & -1.73558300 \\
\hline C & -5.74225000 & 1.54887100 & 2.16004700 & C & 6.59841800 & -1.69210900 & 0.72546100 \\
\hline $\mathrm{H}$ & -4.93616900 & 1.31513300 & 2.85243200 & $\mathrm{H}$ & 6.40108100 & -1.01667100 & 1.55747600 \\
\hline $\mathrm{H}$ & -6.44859200 & 0.71103100 & 2.13589700 & $\mathrm{H}$ & 7.23995500 & -1.18491000 & -0.00757100 \\
\hline $\mathrm{H}$ & -6.26649200 & 2.44838700 & 2.50428300 & $\mathrm{H}$ & 7.12946200 & -2.57558300 & 1.10298400 \\
\hline
\end{tabular}




\begin{tabular}{|c|c|c|c|c|c|c|c|}
\hline $\mathrm{C}$ & -6.09155400 & 2.08905900 & -0.11874600 & $\mathrm{C}$ & 5.47581700 & -2.93562200 & -0.93616500 \\
\hline $\mathrm{H}$ & -6.81530600 & 2.82499600 & 0.25839000 & $\mathrm{H}$ & 6.12482200 & -3.78126800 & -0.66521000 \\
\hline $\mathrm{H}$ & -6.65222000 & 1.19079900 & -0.41277700 & $\mathrm{H}$ & 5.93554500 & -2.41449200 & -1.78832200 \\
\hline C & -5.39207000 & 2.69386600 & -1.31453200 & C & 4.11867200 & -3.47676700 & -1.32461700 \\
\hline $\mathrm{H}$ & -6.14965500 & 2.94365100 & -2.07113000 & $\mathrm{H}$ & 4.25428300 & -4.20057700 & -2.14301000 \\
\hline $\mathrm{H}$ & -4.88797000 & 3.62450500 & -1.02318200 & $\mathrm{H}$ & 3.67012100 & -4.00851700 & -0.47395200 \\
\hline C & -3.87059900 & 2.27030100 & -3.06077700 & C & 2.01668800 & -2.85953200 & -2.22409900 \\
\hline $\mathrm{H}$ & -3.34629500 & 3.21901900 & -2.89431500 & $\mathrm{H}$ & 1.51074800 & -3.48661000 & -1.48319300 \\
\hline $\mathrm{H}$ & -4.63741000 & 2.41969700 & -3.83039500 & $\mathrm{H}$ & 2.13967400 & -3.42838500 & -3.15464500 \\
\hline $\mathrm{H}$ & -3.15758300 & 1.52474700 & -3.40770100 & $\mathrm{H}$ & 1.41549800 & -1.96944600 & -2.40501500 \\
\hline $\mathrm{N}$ & 2.23836100 & 2.59962800 & -3.72560700 & $\mathrm{~N}$ & -2.69209200 & -3.70315200 & -2.64172600 \\
\hline C & 1.24317700 & 3.66263100 & -3.59083900 & C & -2.04306800 & -4.82205500 & -1.96501900 \\
\hline $\mathrm{H}$ & 1.57557200 & 4.40437300 & -2.86841100 & $\mathrm{H}$ & -2.56742500 & -5.06207500 & -1.04234700 \\
\hline $\mathrm{H}$ & 0.26613400 & 3.27451700 & -3.26546500 & $\mathrm{H}$ & -0.99303100 & -4.60660600 & -1.71441900 \\
\hline C & 1.81581200 & 1.59047700 & -4.69764700 & C & -1.97518800 & -3.33779300 & -3.86026000 \\
\hline $\mathrm{H}$ & 0.85853300 & 1.12636500 & -4.41992800 & $\mathrm{H}$ & -0.92696000 & -3.06478900 & -3.66364500 \\
\hline $\mathrm{H}$ & 2.56424700 & 0.80584000 & -4.78174800 & $\mathrm{H}$ & -2.45943100 & -2.49198700 & -4.34386400 \\
\hline $\mathrm{N}$ & 4.28530300 & 1.14651800 & -2.99280200 & $\mathrm{~N}$ & -4.18429300 & -1.56357300 & -2.81395900 \\
\hline C & 4.93202100 & 0.11489400 & -2.18851000 & C & -4.45957600 & -0.15226500 & -2.59678100 \\
\hline $\mathrm{H}$ & 4.24620800 & -0.24540200 & -1.42537400 & $\mathrm{H}$ & -3.68214600 & 0.27718100 & -1.96974100 \\
\hline $\mathrm{H}$ & 5.84343900 & 0.48477800 & -1.69677100 & $\mathrm{H}$ & -5.43256900 & 0.01475800 & -2.10762500 \\
\hline C & 5.13800600 & 1.64551500 & -4.06891300 & C & -5.17964900 & -2.21125800 & -3.65435800 \\
\hline $\mathrm{H}$ & 6.05967900 & 2.10890500 & -3.69159300 & $\mathrm{H}$ & -6.18864400 & -2.19033600 & -3.21412700 \\
\hline $\mathrm{H}$ & 4.60549800 & 2.37436600 & -4.67602200 & $\mathrm{H}$ & -4.90601600 & -3.24766200 & -3.84266000 \\
\hline $\mathrm{N}$ & 3.56077400 & 3.53593100 & -1.66913800 & $\mathrm{~N}$ & -4.27136000 & -3.30307600 & -0.59800600 \\
\hline C & 4.38009800 & 4.46157100 & -2.44710700 & C & -5.26191500 & -4.25854900 & -1.07041700 \\
\hline $\mathrm{H}$ & 4.13874200 & 4.39224300 & -3.50599500 & $\mathrm{H}$ & -4.94128800 & -4.71110100 & -2.00673100 \\
\hline $\mathrm{H}$ & 5.45618500 & 4.28022800 & -2.31944700 & $\mathrm{H}$ & -6.25338900 & -3.80478300 & -1.22313700 \\
\hline C & 3.76562000 & 3.66151400 & -0.23098300 & C & -4.62546600 & -2.71321500 & 0.68381400 \\
\hline $\mathrm{H}$ & 4.79565000 & 3.41753600 & 0.06800500 & $\mathrm{H}$ & -5.58339500 & -2.17012100 & 0.64489000 \\
\hline $\mathrm{H}$ & 3.08085000 & 3.00229300 & 0.29673300 & $\mathrm{H}$ & -3.84650400 & -2.01902300 & 0.98910500 \\
\hline $\mathrm{N}$ & -1.91343800 & -2.42894600 & -3.19189000 & $\mathrm{~N}$ & 2.64352100 & 1.74905500 & -3.47688800 \\
\hline C & -2.34795900 & -3.57038100 & -3.99719800 & C & 3.11879800 & 2.80602100 & -4.36242800 \\
\hline $\mathrm{H}$ & -2.28447700 & -4.49110600 & -3.42179400 & $\mathrm{H}$ & 2.69078700 & 3.76329700 & -4.07495800 \\
\hline $\mathrm{H}$ & -1.75662900 & -3.69022800 & -4.91556100 & $\mathrm{H}$ & 2.87730500 & 2.61915800 & -5.42019000 \\
\hline C & -1.97484500 & -1.17849800 & -3.94169900 & C & 3.16812300 & 0.44928300 & -3.86669100 \\
\hline $\mathrm{H}$ & -1.34282200 & -1.19511800 & -4.84265900 & $\mathrm{H}$ & 2.83569700 & 0.13834600 & -4.87139900 \\
\hline $\mathrm{H}$ & -1.64651300 & -0.35619800 & -3.30778800 & $\mathrm{H}$ & 2.86357800 & -0.31332900 & -3.15557400 \\
\hline $\mathrm{N}$ & 0.79332000 & -3.09985100 & -2.79969500 & $\mathrm{~N}$ & -0.11481200 & 1.72982300 & -4.00060000 \\
\hline C & 0.86543600 & -4.35911200 & -3.53874900 & C & -0.08044200 & 2.63402900 & -5.13925400 \\
\hline $\mathrm{H}$ & 0.10786900 & -5.05736300 & -3.19106300 & $\mathrm{H}$ & 0.41938200 & 3.56468200 & -4.87719900 \\
\hline $\mathrm{H}$ & 1.84795900 & -4.81926400 & -3.38370500 & $\mathrm{H}$ & -1.10434000 & 2.87894100 & -5.44922800 \\
\hline C & 1.81626800 & -2.15189300 & -3.22499300 & C & -0.86843600 & 0.51226100 & -4.25856000 \\
\hline $\mathrm{H}$ & 2.81230600 & -2.56614900 & -3.03314100 & $\mathrm{H}$ & -1.90172700 & 0.76118700 & -4.52851100 \\
\hline $\mathrm{H}$ & 1.72975600 & -1.20923500 & -2.68931300 & $\mathrm{H}$ & -0.89063800 & -0.11310400 & -3.36938800 \\
\hline $\mathrm{N}$ & -1.10925200 & -3.98826100 & -1.23868500 & $\mathrm{~N}$ & 1.00413500 & 3.43313600 & -2.33861400 \\
\hline C & -0.12443600 & -4.63420400 & -0.36687200 & C & -0.31336400 & 3.99631700 & -2.02707800 \\
\hline $\mathrm{H}$ & -0.05233600 & -4.14034700 & 0.61144800 & $\mathrm{H}$ & -0.68130300 & 3.68479000 & -1.04121300 \\
\hline $\mathrm{H}$ & 0.86112400 & -4.62166000 & -0.82432600 & $\mathrm{H}$ & -1.04085400 & 3.68777700 & -2.77464100 \\
\hline C & -2.43811200 & -3.99955900 & -0.63169400 & C & 1.99972000 & 3.86466100 & -1.35917100 \\
\hline $\mathrm{H}$ & -3.15764200 & -3.47509600 & -1.25540500 & $\mathrm{H}$ & 2.96338900 & 3.39918700 & -1.56123400 \\
\hline $\mathrm{H}$ & -2.43758400 & -3.52439400 & 0.36092200 & $\mathrm{H}$ & 1.70406800 & 3.60839400 & -0.33078300 \\
\hline $\mathrm{N}$ & 2.21304700 & -1.22632600 & 1.25605700 & $\mathrm{~N}$ & -1.85165000 & 1.77155700 & 0.95168500 \\
\hline $\mathrm{N}$ & -0.46925000 & 1.23338700 & 1.23651500 & $\mathrm{~N}$ & 0.79731300 & -0.95488600 & 1.61119500 \\
\hline $\mathrm{P}$ & 3.01875800 & -2.05403600 & 2.30863900 & $\mathrm{P}$ & -2.70937600 & 2.83844400 & 1.65669700 \\
\hline $\mathrm{P}$ & -0.63689500 & 2.55875000 & 2.04597300 & $\mathrm{P}$ & 0.74346800 & -2.02481500 & 2.72711400 \\
\hline
\end{tabular}




\begin{tabular}{|c|c|c|c|c|c|c|c|}
\hline $\mathrm{O}$ & -5.14516700 & -1.76705200 & -0.88908600 & $\mathrm{O}$ & 5.21006000 & 1.90747100 & -0.33325600 \\
\hline $\mathrm{O}$ & -4.44463200 & -1.78161000 & 1.85513800 & $\mathrm{O}$ & 3.59170100 & 1.81572100 & 1.95275200 \\
\hline C & -5.74157900 & -1.54970300 & -2.16026900 & $\mathrm{C}$ & 6.24064100 & 1.79801000 & -1.29772100 \\
\hline $\mathrm{H}$ & -4.93532500 & -1.31712700 & -2.85284700 & $\mathrm{H}$ & 5.78826900 & 1.39568200 & -2.20287000 \\
\hline $\mathrm{H}$ & -6.44700300 & -0.71108800 & -2.13636700 & $\mathrm{H}$ & 7.04146700 & 1.12579700 & -0.96149800 \\
\hline $\mathrm{H}$ & -6.26681900 & -2.44880000 & -2.50408300 & $\mathrm{H}$ & 6.67792700 & 2.77921300 & -1.52270200 \\
\hline C & -6.09103900 & -2.08911900 & 0.11870300 & C & 5.65378800 & 2.44091100 & 0.90510700 \\
\hline $\mathrm{H}$ & -6.81531500 & -2.82468500 & -0.25815400 & $\mathrm{H}$ & 6.31301400 & 3.30434700 & 0.73486400 \\
\hline $\mathrm{H}$ & -6.65108000 & -1.19041400 & 0.41256100 & $\mathrm{H}$ & 6.22753400 & 1.68375000 & 1.45934200 \\
\hline C & -5.39180000 & -2.69405800 & 1.31456500 & C & 4.46260800 & 2.90329900 & 1.71449100 \\
\hline $\mathrm{H}$ & -6.14945500 & -2.94333300 & 2.07126200 & $\mathrm{H}$ & 4.82322100 & 3.32659400 & 2.66486400 \\
\hline $\mathrm{H}$ & -4.88818200 & -3.62499600 & 1.02333300 & $\mathrm{H}$ & 3.93271200 & 3.69747400 & 1.17081800 \\
\hline C & -3.86972600 & -2.27112900 & 3.06043100 & C & 2.44194500 & 2.18562700 & 2.71119800 \\
\hline $\mathrm{H}$ & -3.34581600 & -3.22003500 & 2.89380300 & $\mathrm{H}$ & 1.91847500 & 3.02409200 & 2.23836700 \\
\hline $\mathrm{H}$ & -4.63640400 & -2.42027300 & 3.83023200 & $\mathrm{H}$ & 2.72513800 & 2.46969700 & 3.73281100 \\
\hline $\mathrm{H}$ & -3.15634600 & -1.52585700 & 3.40721200 & $\mathrm{H}$ & 1.78063700 & 1.32059100 & 2.72889400 \\
\hline $\mathrm{N}$ & 2.23860400 & -2.59937800 & 3.72562300 & $\mathrm{~N}$ & -1.98764100 & 4.05030100 & 2.65839700 \\
\hline C & 1.24361500 & -3.66256400 & 3.59086000 & C & -1.14788300 & 5.04051800 & 1.99134400 \\
\hline $\mathrm{H}$ & 1.57610800 & -4.40420700 & 2.86837600 & $\mathrm{H}$ & -1.61267900 & 5.36427200 & 1.06248700 \\
\hline $\mathrm{H}$ & 0.26648300 & -3.27461600 & 3.26556000 & $\mathrm{H}$ & -0.14657000 & 4.64904000 & 1.75349300 \\
\hline C & 1.81589100 & -1.59031500 & 4.69767800 & C & -1.37836600 & 3.59591000 & 3.90478500 \\
\hline $\mathrm{H}$ & 0.85853100 & -1.12636200 & 4.41997100 & $\mathrm{H}$ & -0.37969100 & 3.16059600 & 3.74854200 \\
\hline $\mathrm{H}$ & 2.56419300 & -0.80555200 & 4.78178700 & $\mathrm{H}$ & -2.00409100 & 2.84232000 & 4.37840100 \\
\hline $\mathrm{N}$ & 4.28533200 & -1.14593700 & 2.99288100 & $\mathrm{~N}$ & -3.81635100 & 2.19060900 & 2.80378900 \\
\hline C & 4.93186900 & -0.11415800 & 2.18864200 & C & -4.28885500 & 0.82733600 & 2.62563900 \\
\hline $\mathrm{H}$ & 4.24600200 & 0.24603700 & 1.42550600 & $\mathrm{H}$ & -3.57966200 & 0.27455500 & 2.01421800 \\
\hline $\mathrm{H}$ & 5.84336500 & -0.48385000 & 1.69690400 & $\mathrm{H}$ & -5.27516600 & 0.78667300 & 2.13724000 \\
\hline C & 5.13811400 & -1.64482200 & 4.06898100 & C & -4.70079500 & 2.99656500 & 3.63017700 \\
\hline $\mathrm{H}$ & 6.05987900 & -2.10802100 & 3.69165200 & $\mathrm{H}$ & -5.70178600 & 3.11938000 & 3.18872600 \\
\hline $\mathrm{H}$ & 4.60573400 & -2.37380100 & 4.67604900 & $\mathrm{H}$ & -4.27387300 & 3.98311900 & 3.79941500 \\
\hline $\mathrm{N}$ & 3.56122700 & -3.53548200 & 1.66920700 & $\mathrm{~N}$ & -3.53701500 & 3.88239300 & 0.56376900 \\
\hline C & 4.38065300 & -4.46100100 & 2.44721400 & C & -4.41575600 & 4.95758200 & 0.99752800 \\
\hline $\mathrm{H}$ & 4.13920600 & -4.39174300 & 3.50608500 & $\mathrm{H}$ & -4.07765700 & 5.36799100 & 1.94723500 \\
\hline $\mathrm{H}$ & 5.45671900 & -4.27947000 & 2.31964000 & $\mathrm{H}$ & -5.46310800 & 4.63618900 & 1.10810600 \\
\hline C & 3.76623200 & -3.66098000 & 0.23106500 & C & -3.88281100 & 3.36504900 & -0.75079600 \\
\hline $\mathrm{H}$ & 4.79623300 & -3.41675900 & -0.06782600 & $\mathrm{H}$ & -4.89784500 & 2.93771900 & -0.78244400 \\
\hline $\mathrm{H}$ & 3.08135500 & -3.00190600 & -0.29669500 & $\mathrm{H}$ & -3.17254000 & 2.59143400 & -1.03167000 \\
\hline $\mathrm{N}$ & -1.91415700 & 2.42854200 & 3.19163200 & $\mathrm{~N}$ & 2.30394500 & -2.53221400 & 3.28391200 \\
\hline C & -2.34928500 & 3.56988500 & 3.99674100 & C & 2.55272500 & -3.69567300 & 4.12251000 \\
\hline $\mathrm{H}$ & -2.28591400 & 4.49059800 & 3.42130700 & $\mathrm{H}$ & 1.75499500 & -4.42542600 & 4.00952000 \\
\hline $\mathrm{H}$ & -1.75827300 & 3.68996800 & 4.91527800 & $\mathrm{H}$ & 2.63984300 & -3.43610700 & 5.18903400 \\
\hline C & -1.97534400 & 1.17812700 & 3.94152100 & C & 3.35138900 & -1.53602700 & 3.41205600 \\
\hline $\mathrm{H}$ & -1.34365500 & 1.19508500 & 4.84270700 & $\mathrm{H}$ & 3.48844600 & -1.20699400 & 4.45529700 \\
\hline $\mathrm{H}$ & -1.64644800 & 0.35590200 & 3.30780200 & $\mathrm{H}$ & 3.11569400 & -0.65943300 & 2.81511800 \\
\hline $\mathrm{N}$ & 0.79256400 & 3.09996200 & 2.80009200 & $\mathrm{~N}$ & -0.20564400 & -1.57243300 & 4.08654100 \\
\hline C & 0.86419300 & 4.35908900 & 3.53941500 & C & -0.22656900 & -2.36412300 & 5.30599400 \\
\hline $\mathrm{H}$ & 0.10652900 & 5.05722200 & 3.19169500 & $\mathrm{H}$ & -0.09597100 & -3.42112000 & 5.08039300 \\
\hline $\mathrm{H}$ & 1.84663300 & 4.81952400 & 3.38468600 & $\mathrm{H}$ & -1.19592800 & -2.24751800 & 5.80590400 \\
\hline C & 1.81573500 & 2.15220900 & 3.22531000 & C & -0.54336500 & -0.17492600 & 4.30357000 \\
\hline $\mathrm{H}$ & 2.81167700 & 2.56679400 & 3.03366300 & $\mathrm{H}$ & -1.53238900 & -0.10156300 & 4.77150200 \\
\hline $\mathrm{H}$ & 1.72954400 & 1.20964900 & 2.68940900 & $\mathrm{H}$ & -0.57961200 & 0.35617500 & 3.35546400 \\
\hline $\mathrm{N}$ & -1.10984300 & 3.98808400 & 1.23867400 & $\mathrm{~N}$ & 0.08406200 & -3.59417000 & 2.44853700 \\
\hline C & -0.12493500 & 4.63436100 & 0.36721200 & C & -1.37236900 & -3.71509500 & 2.34340300 \\
\hline $\mathrm{H}$ & -0.05233900 & 4.14055300 & -0.61109700 & $\mathrm{H}$ & -1.74695400 & -3.40499300 & 1.35978600 \\
\hline $\mathrm{H}$ & 0.86046900 & 4.62212300 & 0.82500900 & $\mathrm{H}$ & -1.85295000 & -3.10532800 & 3.10549500 \\
\hline C & -2.43850300 & 3.99899800 & 0.63124700 & C & 0.74878300 & -4.40030600 & 1.42905500 \\
\hline
\end{tabular}




\begin{tabular}{|c|c|c|c|c|c|c|c|}
\hline $\mathrm{H}$ & -3.15807200 & 3.47428900 & 1.25471600 & $\mathrm{H}$ & 1.82854700 & -4.26495700 & 1.48243800 \\
\hline $\mathrm{H}$ & -2.43750000 & 3.52388100 & -0.36139600 & $\mathrm{H}$ & 0.41397300 & -4.13965100 & 0.41530600 \\
\hline $\mathrm{H}$ & 5.42947000 & -0.81200600 & 4.71868000 & $\mathrm{H}$ & -4.83475300 & 2.51539700 & 4.60811300 \\
\hline $\mathrm{H}$ & 5.21826300 & 0.72710200 & 2.82979500 & $\mathrm{H}$ & -4.37980200 & 0.32897700 & 3.59966300 \\
\hline $\mathrm{H}$ & 4.17790400 & -5.48753700 & 2.12325600 & $\mathrm{H}$ & -4.40101800 & 5.76719000 & 0.25659800 \\
\hline $\mathrm{H}$ & 1.10713600 & -4.15423300 & 4.55885900 & $\mathrm{H}$ & -1.02241000 & 5.91233100 & 2.64362700 \\
\hline $\mathrm{H}$ & 1.69430500 & -2.06119500 & 5.67793000 & $\mathrm{H}$ & -1.27207800 & 4.44406600 & 4.59083900 \\
\hline $\mathrm{H}$ & -2.77023200 & -5.03560100 & -0.50686300 & $\mathrm{H}$ & 2.11806300 & 4.95309500 & -1.41378000 \\
\hline $\mathrm{H}$ & -0.42054600 & -5.67512100 & -0.20480000 & $\mathrm{H}$ & -0.24413100 & 5.08965200 & -2.04162400 \\
\hline $\mathrm{H}$ & -3.39212500 & -3.42646600 & -4.29604500 & $\mathrm{H}$ & 4.21185200 & 2.88228900 & -4.28940500 \\
\hline $\mathrm{H}$ & -3.00380600 & -0.99210700 & -4.27028100 & $\mathrm{H}$ & 4.26554200 & 0.47923200 & -3.88841800 \\
\hline $\mathrm{H}$ & 0.73789800 & -4.21197300 & -4.61977500 & $\mathrm{H}$ & 0.42858400 & 2.19719400 & -6.01244500 \\
\hline $\mathrm{H}$ & 1.74844800 & -1.93561300 & -4.30079500 & $\mathrm{H}$ & -0.43981200 & -0.07317700 & -5.08796600 \\
\hline $\mathrm{H}$ & 5.42952700 & 0.81272200 & -4.71856800 & $\mathrm{H}$ & -5.23738800 & -1.69645200 & -4.62223100 \\
\hline $\mathrm{H}$ & 5.21858600 & -0.72633800 & -2.82962400 & $\mathrm{H}$ & -4.47589000 & 0.38023800 & -3.55638500 \\
\hline $\mathrm{H}$ & 1.10655400 & 4.15422700 & -4.55885400 & $\mathrm{H}$ & -2.05860100 & -5.70316800 & -2.61682300 \\
\hline $\mathrm{H}$ & 1.69414300 & 2.06132300 & -5.67790400 & $\mathrm{H}$ & -1.97758600 & -4.18643000 & -4.55379200 \\
\hline $\mathrm{H}$ & 4.17714900 & 5.48808100 & -2.12319400 & $\mathrm{H}$ & -5.38284600 & -5.06080700 & -0.33091300 \\
\hline $\mathrm{H}$ & 3.56661000 & 4.69429300 & 0.07631600 & $\mathrm{H}$ & -4.71984500 & -3.49762100 & 1.44549200 \\
\hline $\mathrm{H}$ & 0.73644400 & 4.21170600 & 4.62038400 & $\mathrm{H}$ & 0.55125300 & -2.06154500 & 6.02507000 \\
\hline $\mathrm{H}$ & 1.74784700 & 1.93567900 & 4.30105600 & $\mathrm{H}$ & 0.17567700 & 0.33363200 & 4.96723300 \\
\hline $\mathrm{H}$ & -2.77091200 & 5.03493900 & 0.50635000 & $\mathrm{H}$ & 0.52407800 & -5.46066500 & 1.59367600 \\
\hline $\mathrm{H}$ & -0.42132300 & 5.67518700 & 0.20506600 & $\mathrm{H}$ & -1.65652600 & -4.76129000 & 2.50505200 \\
\hline $\mathrm{H}$ & 3.56748700 & -4.69379700 & -0.07627300 & $\mathrm{H}$ & -3.83916400 & 4.17216600 & -1.49307100 \\
\hline $\mathrm{H}$ & -3.00434800 & 0.99134500 & 4.26974300 & $\mathrm{H}$ & 4.31112800 & -1.94380200 & 3.06722700 \\
\hline $\mathrm{H}$ & -3.39349600 & 3.42565200 & 4.29527900 & $\mathrm{H}$ & 3.49810400 & -4.17161600 & 3.82717200 \\
\hline
\end{tabular}

Table S15. Calculated absolute potentials of the redox couples in THF at the scalar relativistic level $\left(E_{\mathrm{SR}}\right)$ and at the spin-orbit relativistic level $\left(E_{\mathrm{so}}\right)$, respectively. The calculated $E_{\mathrm{SR}}$ for $\mathrm{Fc}^{+} \rightarrow \mathrm{Fc}^{0}$ is $-5.436 \mathrm{~V}$.

$\begin{array}{lll} & E_{\text {SR }}(\mathbf{V}) & \boldsymbol{E}_{\text {so }}(\mathbf{V}) \\ {\left[\mathrm{Ce}\left(\mathrm{NP}\left(\mathrm{NMe}_{2}\right)_{3}\right)_{4}\right]^{0} \rightarrow\left[\mathrm{Ce}\left(\mathrm{NP}\left(\mathrm{NMe}_{2}\right)_{3}\right)_{4}\right]^{-}} & -2.886 & -3.013 \\ {\left[\mathrm{~K}(\mathrm{DME})_{2}\right]\left[\mathrm{Ce}\left(\mathrm{NP}\left(\mathrm{NMe}_{2}\right)_{3}\right)_{4}\right]^{+} \rightarrow} & -3.219 & -3.346 \\ {\left[\mathrm{~K}(\mathrm{DME})_{2}\right]\left[\mathrm{Ce}\left(\mathrm{NP}(\mathrm{NMe})_{3}\right)_{4}\right]^{0}} & & \\ {\left[\mathrm{Ce}\left(\mathrm{NP}(\mathrm{NMe})_{3}\right)_{4}\right]+\mathrm{K}(\mathrm{DME})_{2}{ }^{+} \rightarrow} & -2.324 & -2.451 \\ {\left[\mathrm{~K}(\mathrm{DME})_{2}\right]\left[\mathrm{Ce}\left(\mathrm{NP}(\mathrm{NMe})_{3}\right)_{4}\right]^{0}} & & \\ {\left[\mathrm{Ce}(\mathrm{pyNO})_{4}\right]^{0} \rightarrow\left[\mathrm{Ce}(\text { pyNO })_{4}\right]^{-}} & -3.225 & -3.352\end{array}$




\section{Chemical Reactivity}

Unless otherwise noted, chemical oxidations were performed inside of a J. Young tube in $d_{8}$-THF with excess oxidant or reductant. The reactions were followed by ${ }^{31} \mathrm{P}$ NMR spectroscopy and the cerium and ligand products determined by comparison with isolated materials. Reactions were monitored for conversion on NMR scale. Products were not isolated.

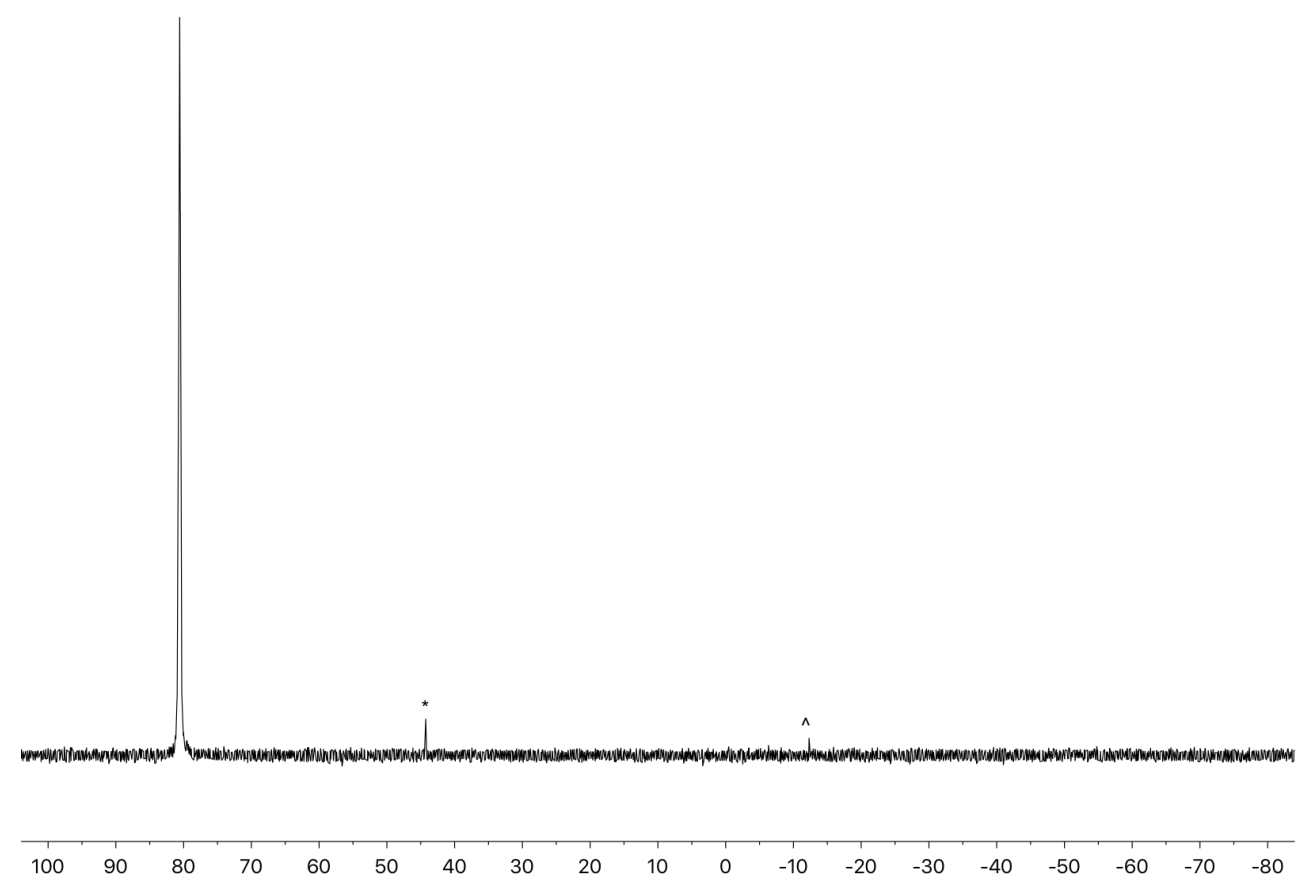

Figure S26. Reaction of 2-Ce with $\mathrm{Na}^{0}$ sand in diethyl ether in $20 \mathrm{~mL}$ scintillation vial overnight, monitored by ${ }^{31} \mathrm{P}$ NMR. Residual imine denoted by *. Residual 2-Ce starting material denoted by ${ }^{\wedge}$. 


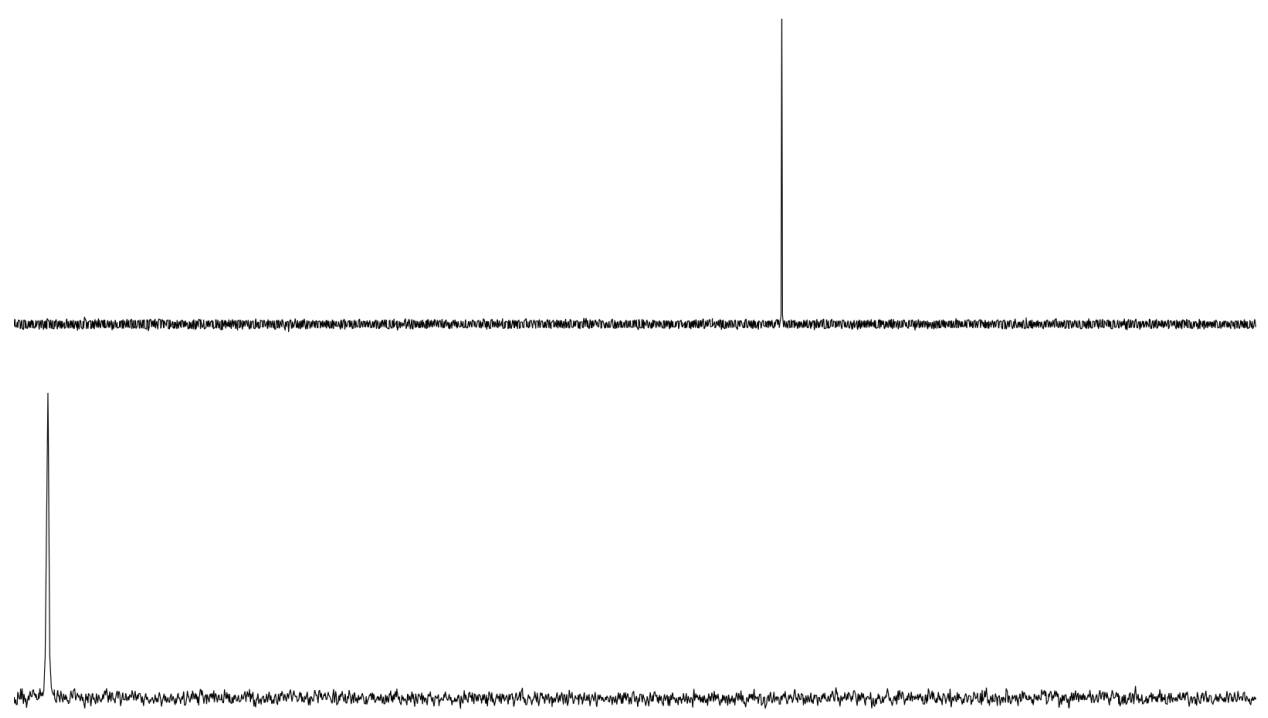

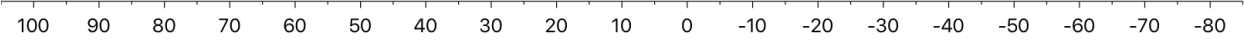

Figure S27. Reaction of 2-Ce with sodium anthranecide, resulting in conversion to $3-$ $\mathrm{Ce}-\mathrm{Na}\left(\mathrm{Et}_{2} \mathrm{O}_{\mathrm{x}}\right)$ (see Fig. S25), shown by ${ }^{31} \mathrm{P}$ NMR.

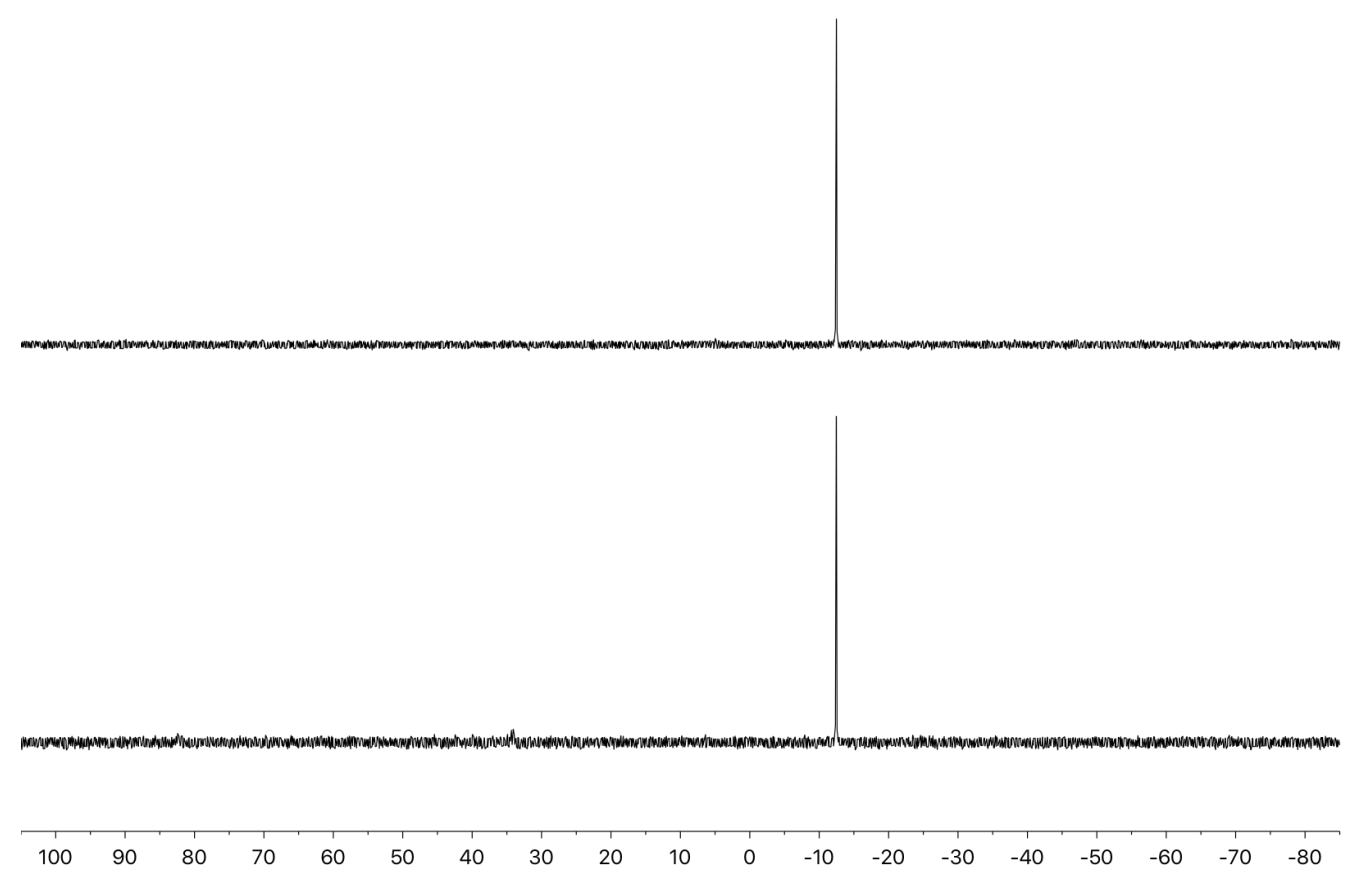

Figure S28. Reaction of 2-Ce with sodium benzophenone, resulting in no conversion to 3-Ce- $\mathrm{Na}\left(\mathrm{Et}_{2} \mathrm{O}_{x}\right)$ (see Fig. S25), shown by ${ }^{31} \mathrm{P} \mathrm{NMR}$. 

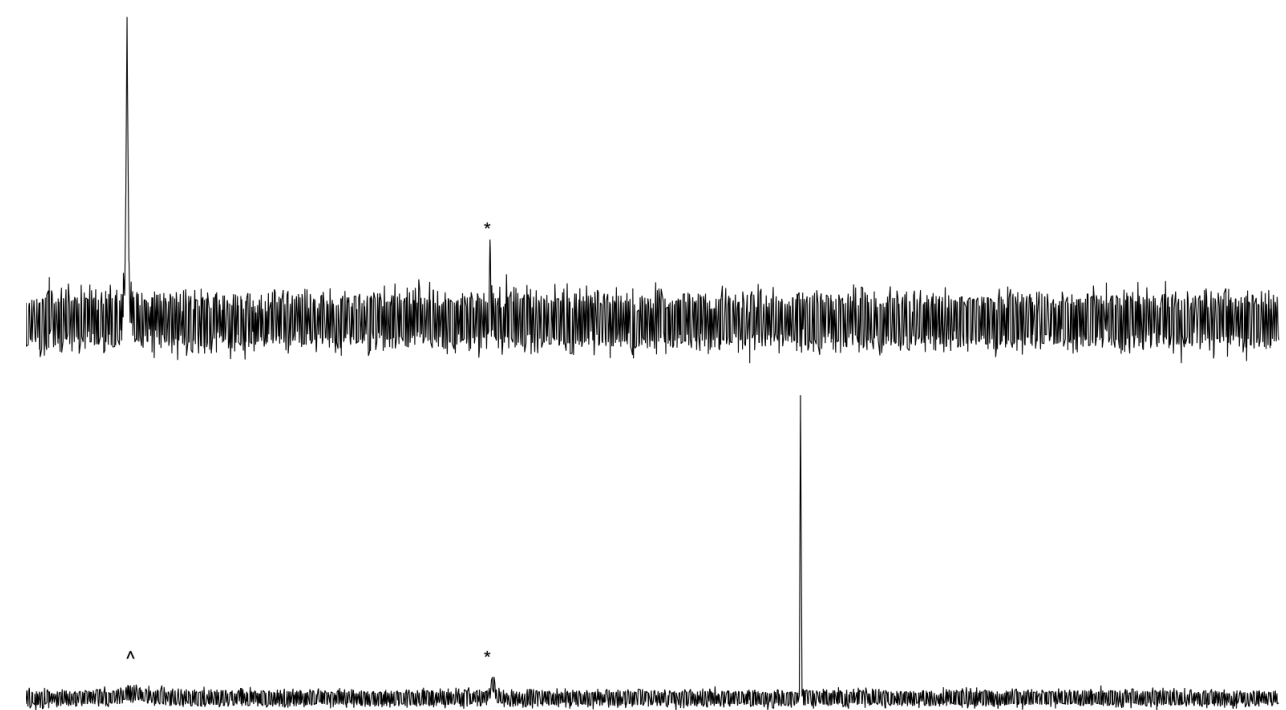

$\begin{array}{lllllllllllllllllll}100 & 90 & 80 & 70 & 60 & 50 & 40 & 30 & 20 & 10 & 0 & -10 & -20 & -30 & -40 & -50 & -60 & -70 & -80\end{array}$

Figure S29. Reaction of 3-Ce-K(Et $\left.{ }_{2} \mathrm{O}\right)$ with anthracene, resulting in conversion to 2 Ce, shown by ${ }^{31} \mathrm{P}$ NMR. Residual imine denoted by *. Residual starting material denoted by ${ }^{\wedge}$.
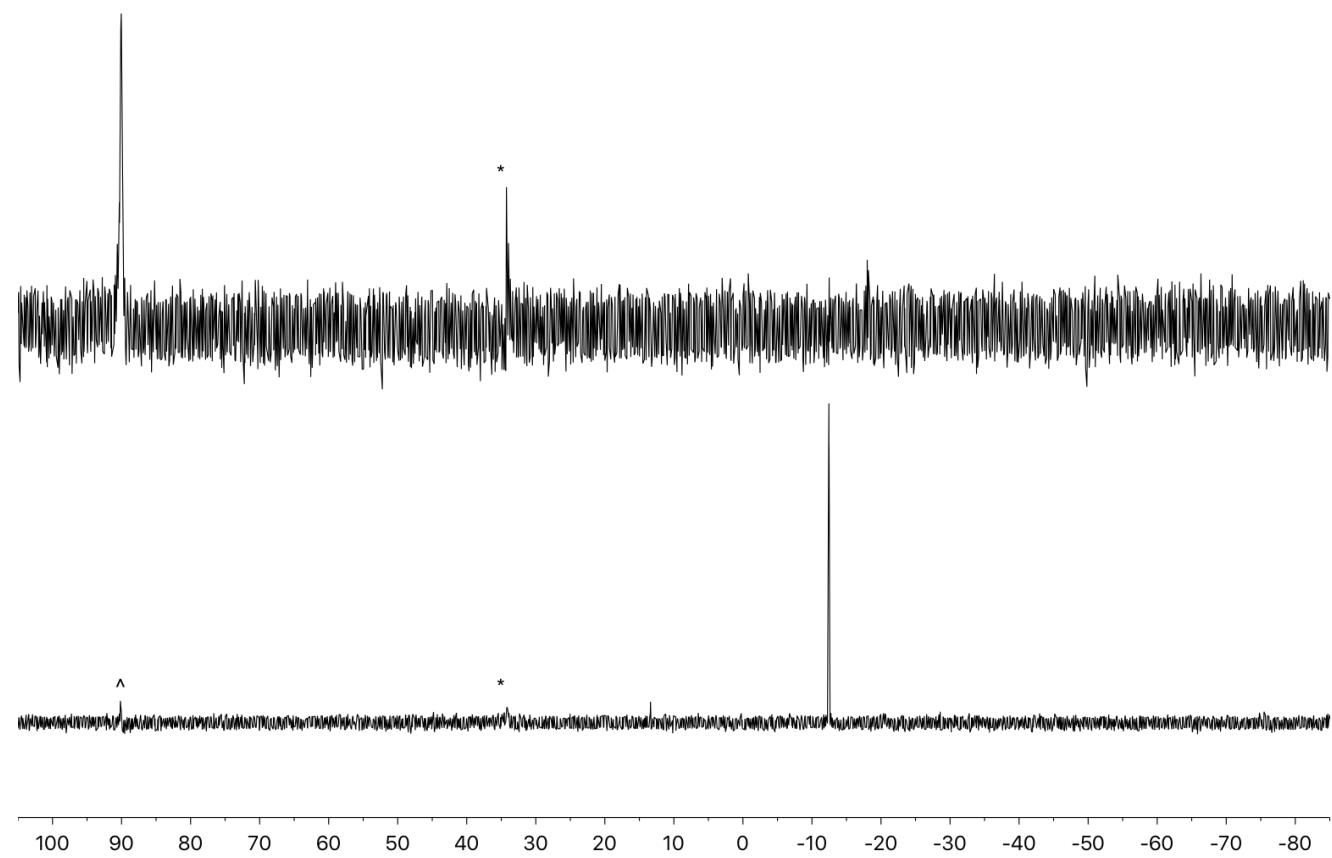

Figure S30. Reaction of 3-Ce- $\mathrm{K}\left(\mathrm{Et}_{2} \mathrm{O}\right)$ with 2,6-di-tert-butylanthracene, resulting in conversion to $2-\mathrm{Ce}$, shown by ${ }^{31} \mathrm{P}$ NMR. Residual imine denoted by *. Residual starting material denoted by ${ }^{\wedge}$. 

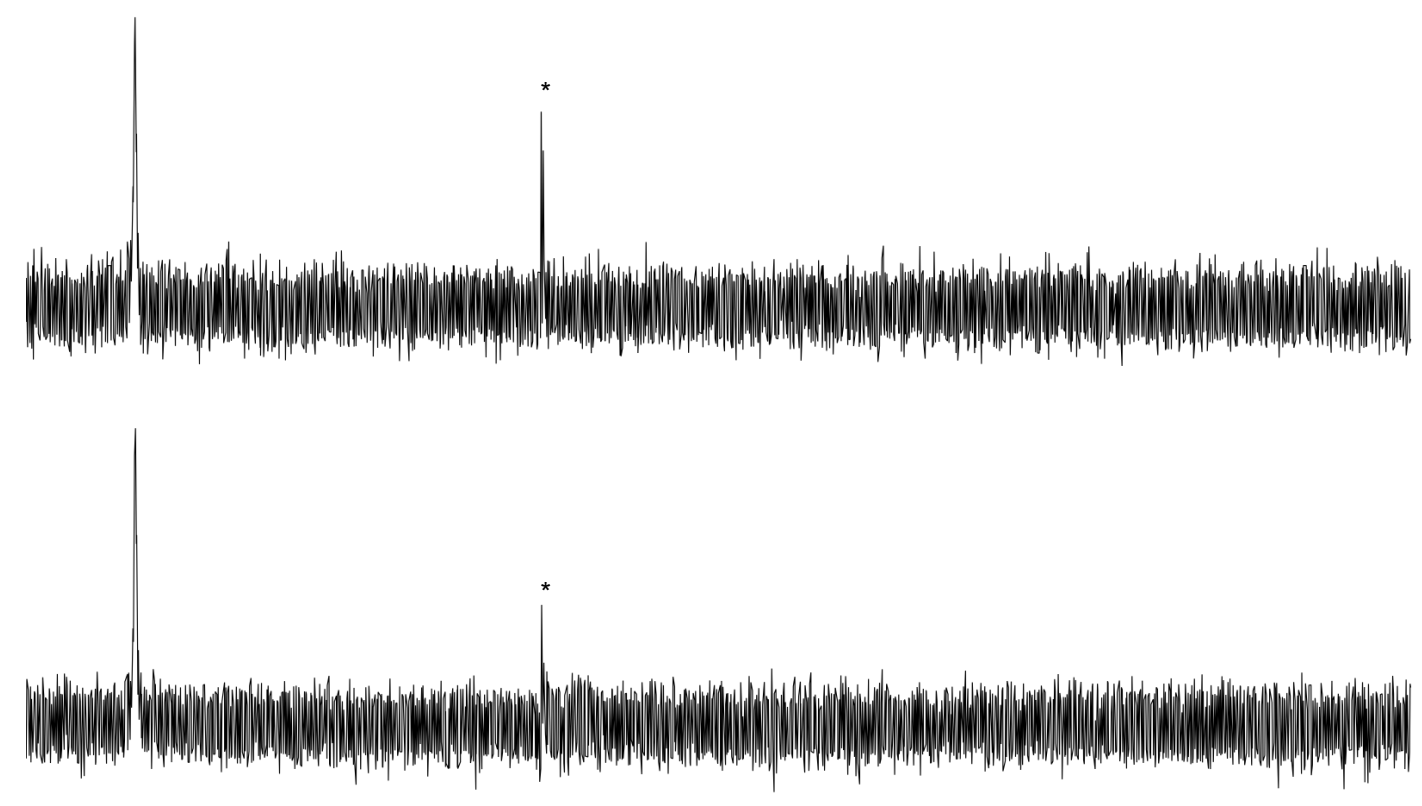

$\begin{array}{lllllllllllllllllll}100 & 90 & 80 & 70 & 60 & 50 & 40 & 30 & 20 & 10 & 0 & -10 & -20 & -30 & -40 & -50 & -60 & -70 & -80\end{array}$

Figure S31. Reaction of 3-Ce-K(Et $\left.\mathbf{t}_{2} \mathrm{O}\right)$ with naphthalene, resulting in no conversion to 2-Ce, shown by ${ }^{31} \mathrm{P}$ NMR. Residual imine denoted by *.
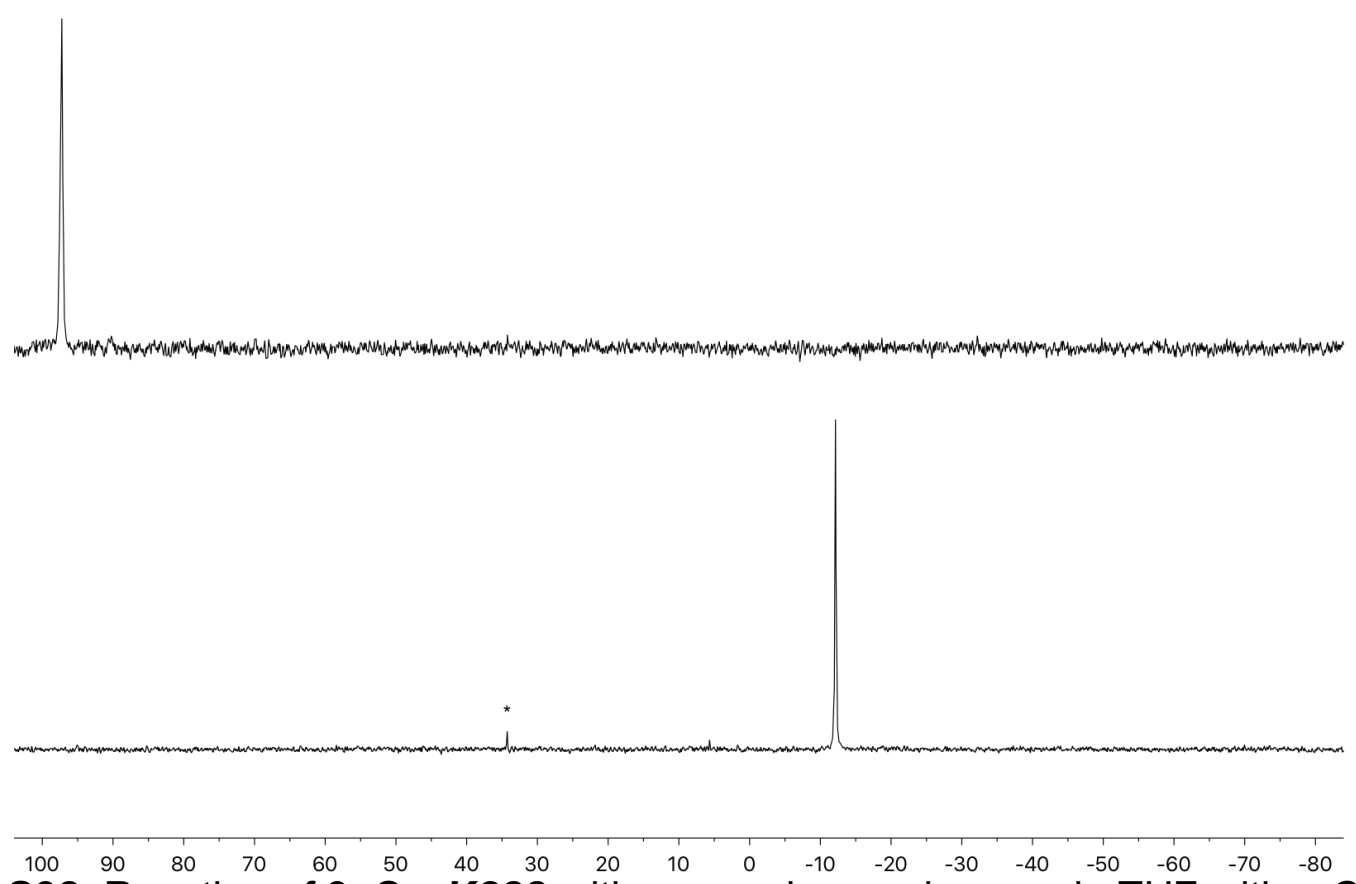

Figure S32. Reaction of 3-Ce-K222 with excess benzophenone in THF with a $\mathrm{C}_{6} \mathrm{D}_{6}$ insert, resulting in conversion to $2-\mathrm{Ce}$, shown by ${ }^{31} \mathrm{P}$ NMR. Residual imine denoted by *. 

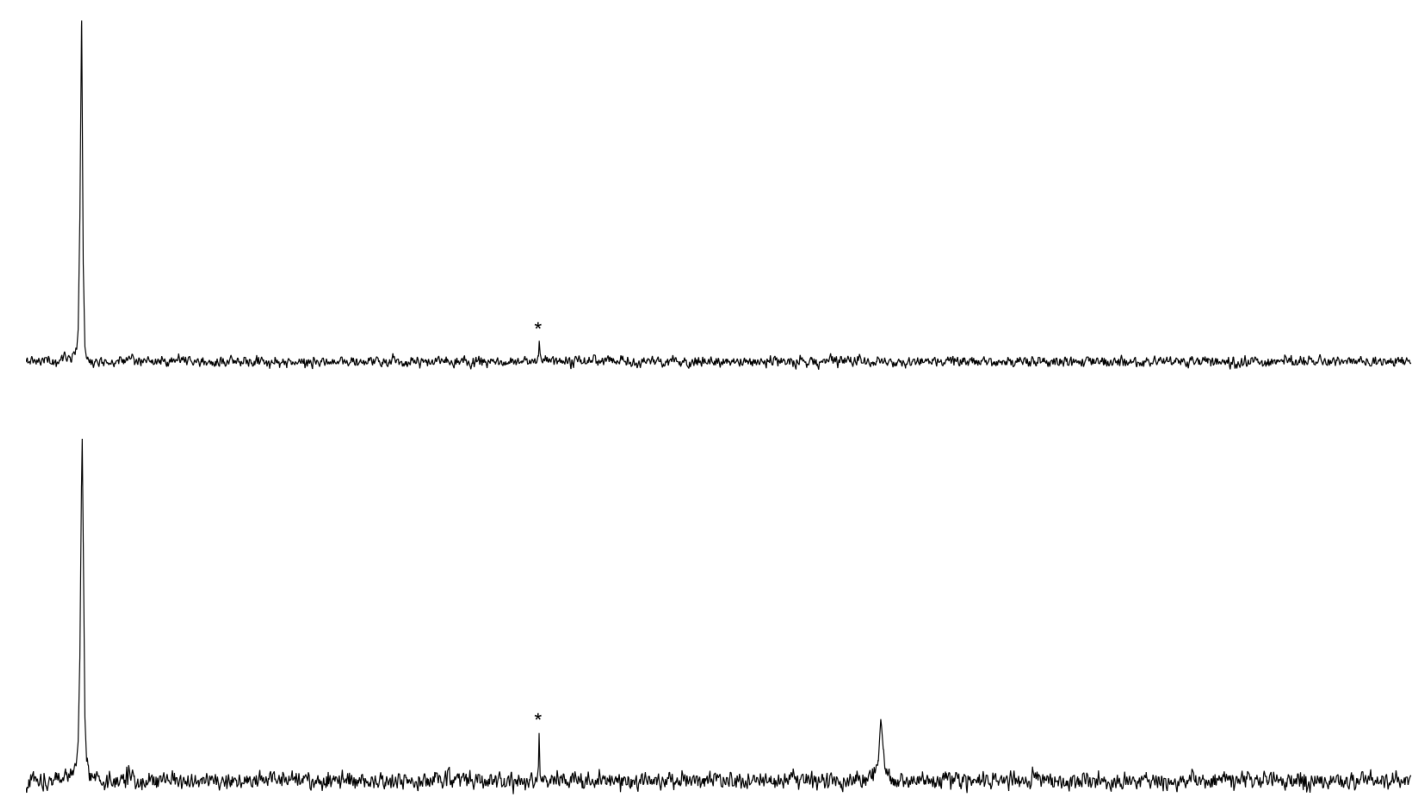

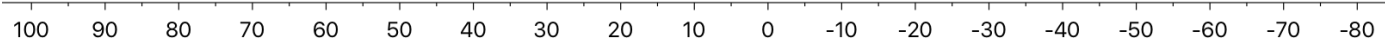

Figure S33. Reaction of 3-Ce-K222 with anthracene (1:1) in THF with a $\mathrm{C}_{6} \mathrm{D}_{6}$ insert, resulting in non-stoichiometric production of $2-\mathrm{Ce}$ and imine, shown by ${ }^{31} \mathrm{P}$ NMR. Residual imine denoted by *.
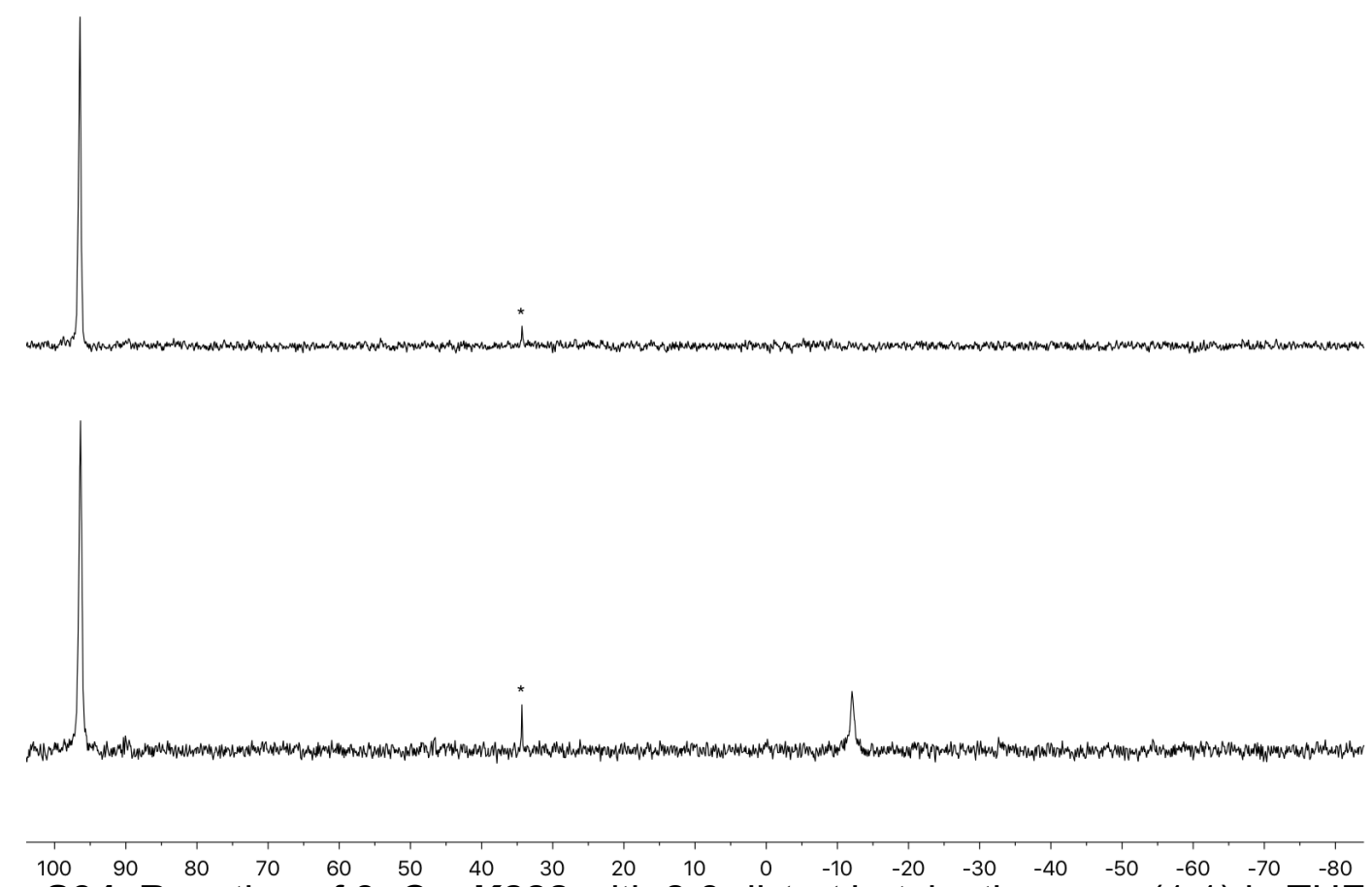

Figure S34. Reaction of 3-Ce-K222 with 2,6-di-tert-butylanthracene (1:1) in THF with a $\mathrm{C}_{6} \mathrm{D}_{6}$ insert, resulting in non-stoichiometric production of $2-\mathrm{Ce}$ and imine, shown by ${ }^{31} \mathrm{P}$ NMR. Residual imine denoted by *. 


\section{Cyclic Voltammetry}

Electrochemical measurements were performed on a Pine Wave Driver 20 Bipotentiostat/Galvanostat. Measurements were performed inside a $\mathrm{N}_{2}$ atmosphere glovebox in a $20 \mathrm{~mL}$ electrochemical cell with a glassy carbon working electrode, a platinum wire counter electrode, and a $\mathrm{Ag} / \mathrm{AgCl}$ pseudo reference electrode. Electrodes were polished before each use. Measurements were performed in a positive feedback IR compensation mode and referenced versus $\mathrm{Fc} / \mathrm{Fc}^{+}$.

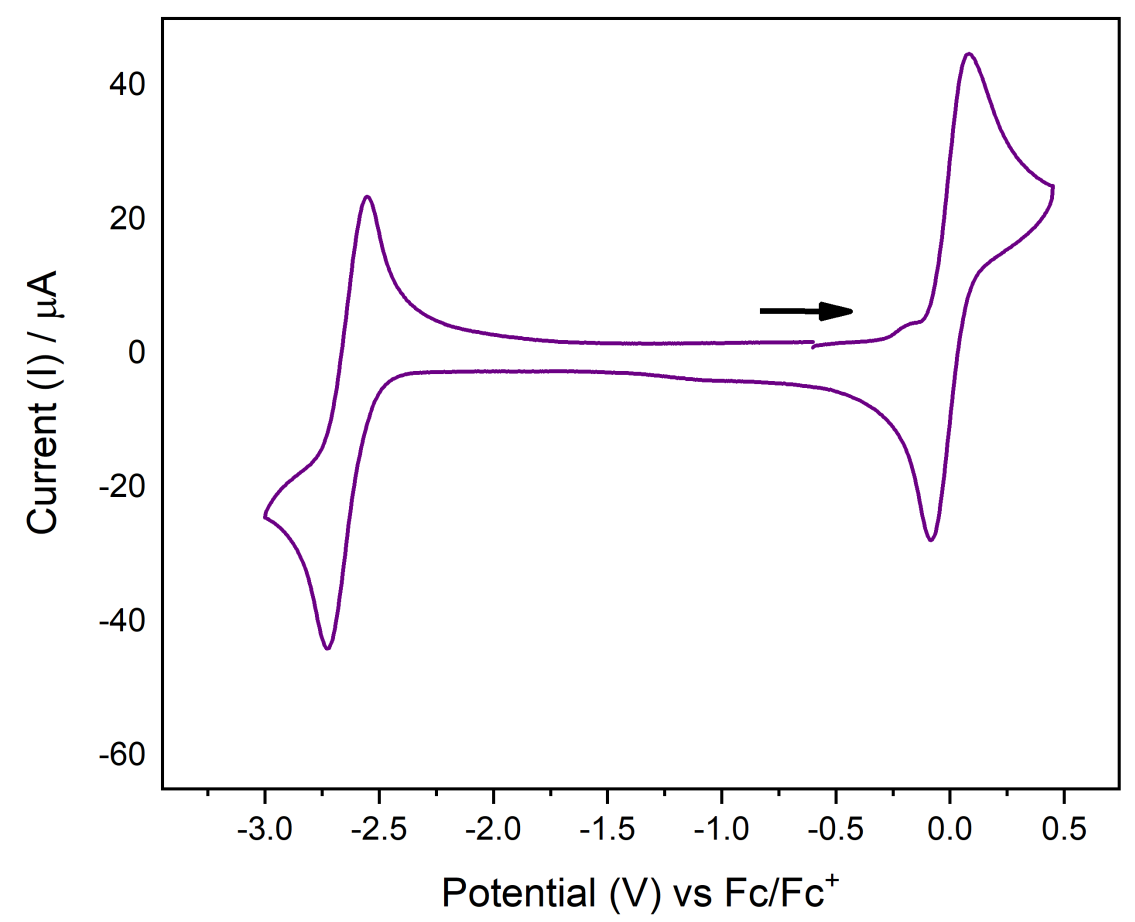

Figure S35. CV of 2,6-di-tert-butylanthracene referenced against ferrocene in THF $(0.1$ $\mathrm{M}\left[\mathrm{NBu}_{4}\right]\left[\mathrm{PF}_{6}\right], 3 \mathrm{mM}$ analyte). 


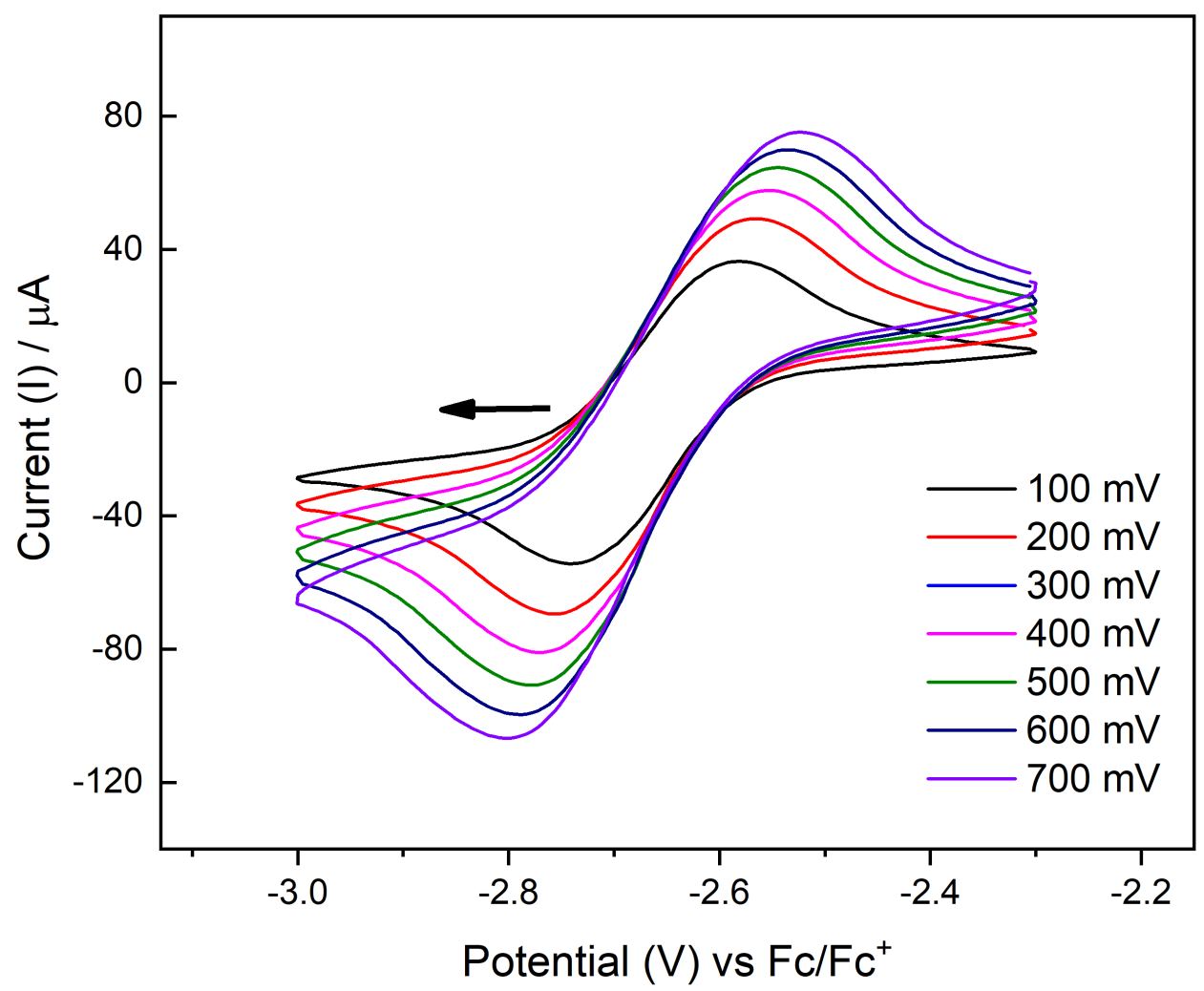

Figure S36. Scan rate dependence of 2,6-di-tert-butylanthracene in THF $(0.1 \mathrm{M}$ $\left[\mathrm{NBu}_{4}\right]\left[\mathrm{PF}_{6}\right], 3 \mathrm{mM}$ analyte).

A

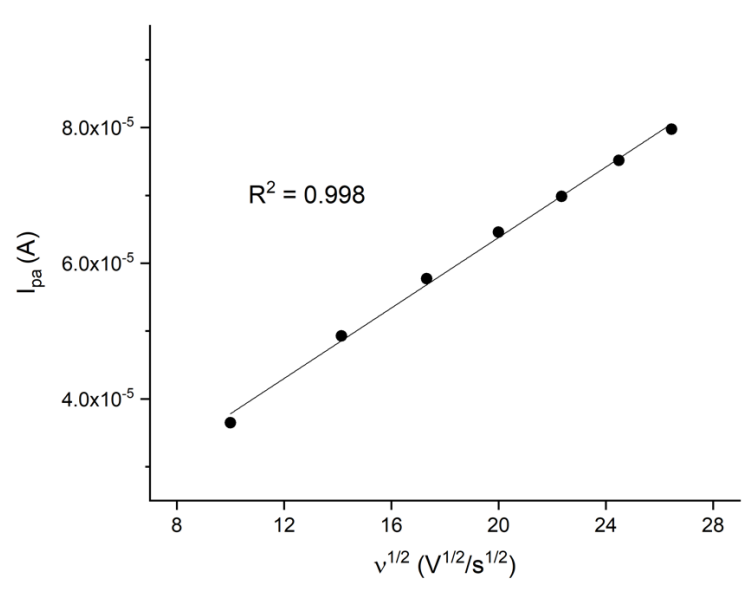

B

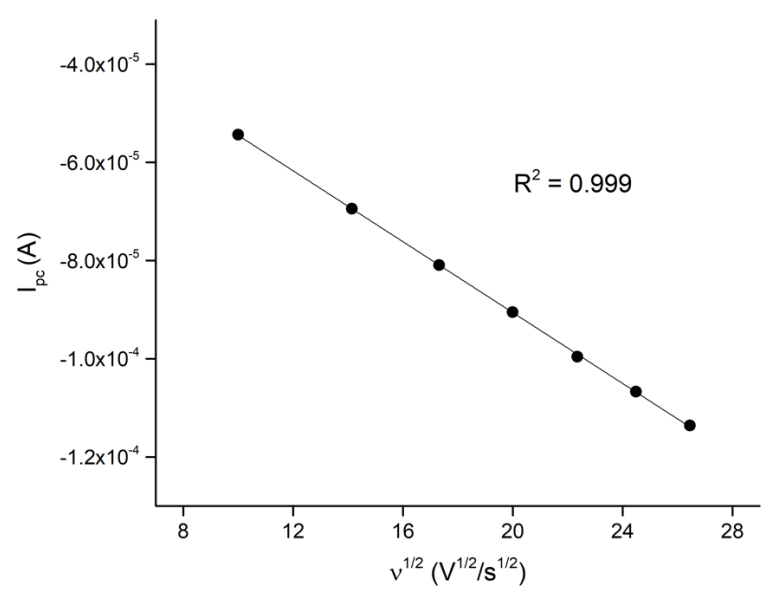

Figure S37. Randals-Sevcik Plots associated with Fig. S35 where $\mathbf{A}$ is the anodic wave and $\mathbf{B}$ is the cathodic wave. 


\section{Ce $L_{3}$-edge XANES}

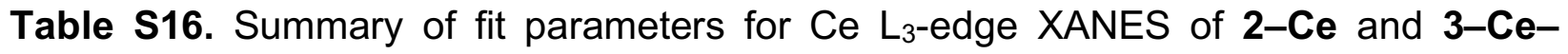
$\mathrm{K}\left(\mathrm{Et}_{2} \mathrm{O}\right)$.

\begin{tabular}{|c|c|c|c|c|c|c|}
\hline \multirow[t]{2}{*}{ Complex } & \multicolumn{2}{|l|}{ Peak 1} & \multicolumn{2}{|l|}{ Peak 2} & \multicolumn{2}{|l|}{ Peak 3} \\
\hline & Intensity & Energy $(\mathrm{eV})$ & Intensity & Energy $(\mathrm{eV})$ & Intensity & Energy $(\mathrm{eV})$ \\
\hline $\begin{array}{l}3-\mathrm{Ce}- \\
\mathrm{K}\left(\mathrm{Et}_{2} \mathrm{O}\right)\end{array}$ & $6.60(3)$ & $5725.2(1)$ & NA & NA & NA & NA \\
\hline $2-\mathrm{Ce}$ & $0.31(2)$ & $5720.1(1)$ & $1.19(5)$ & $5728.2(1)$ & $4.37(6)$ & $5735.2(1)$ \\
\hline
\end{tabular}

Table S17. Normalized and Background Subtracted $\mathrm{L}_{3}$-edge XAS of 2-Ce and 3-Ce$\mathrm{K}\left(\mathrm{Et}_{2} \mathrm{O}\right)$.

$\begin{array}{cccc}\text { 2-Ce_CeL3_eV } & \text { 2-Ce_CeL3_int } & \begin{array}{c}\text { 3-Ce-K(Et2O) } \\ \text { CeL3_eV }\end{array} & \begin{array}{c}\text { 3-Ce-K(Et2O) } \\ \text {-CeL3_int }\end{array} \\ 5522.2 & 4.76 \mathrm{E}-03 & 5522.63 & 8.98 \mathrm{E}-03 \\ 5525.291 & 5.16 \mathrm{E}-03 & 5525.721 & 1.05 \mathrm{E}-02 \\ 5528.382 & 7.17 \mathrm{E}-03 & 5528.812 & 1.03 \mathrm{E}-02 \\ 5531.473 & 5.41 \mathrm{E}-03 & 5531.903 & 8.59 \mathrm{E}-03 \\ 5534.564 & 6.64 \mathrm{E}-03 & 5534.994 & 8.69 \mathrm{E}-03 \\ 5537.655 & 1.50 \mathrm{E}-03 & 5538.085 & 6.75 \mathrm{E}-03 \\ 5540.746 & 6.87 \mathrm{E}-03 & 5541.176 & 6.61 \mathrm{E}-03 \\ 5543.837 & 1.29 \mathrm{E}-03 & 5544.267 & 5.70 \mathrm{E}-03 \\ 5546.928 & 5.30 \mathrm{E}-03 & 5547.358 & 5.95 \mathrm{E}-03 \\ 5550.019 & 3.21 \mathrm{E}-05 & 5550.449 & 4.68 \mathrm{E}-03 \\ 5553.11 & 3.91 \mathrm{E}-03 & 5553.54 & 4.10 \mathrm{E}-03 \\ 5556.201 & -1.22 \mathrm{E}-03 & 5556.631 & 2.26 \mathrm{E}-03 \\ 5559.292 & 3.90 \mathrm{E}-03 & 5559.722 & 2.47 \mathrm{E}-03 \\ 5562.383 & -1.54 \mathrm{E}-03 & 5562.813 & 1.08 \mathrm{E}-03 \\ 5565.474 & 2.57 \mathrm{E}-03 & 5565.904 & 1.31 \mathrm{E}-03 \\ 5568.565 & -2.02 \mathrm{E}-03 & 5568.995 & 2.25 \mathrm{E}-04 \\ 5571.656 & 2.05 \mathrm{E}-03 & 5572.086 & 7.22 \mathrm{E}-04 \\ 5574.747 & -2.50 \mathrm{E}-03 & 5575.177 & -3.39 \mathrm{E}-04 \\ 5577.838 & 1.21 \mathrm{E}-03 & 5578.268 & -5.75 \mathrm{E}-04 \\ 5580.929 & -3.74 \mathrm{E}-03 & 5581.359 & -2.52 \mathrm{E}-03 \\ 5584.02 & 7.48 \mathrm{E}-04 & 5584.45 & -1.66 \mathrm{E}-03 \\ 5587.111 & -4.22 \mathrm{E}-03 & 5587.541 & -2.94 \mathrm{E}-03 \\ 5590.202 & -8.88 \mathrm{E}-04 & 5590.632 & -2.63 \mathrm{E}-03\end{array}$




$\begin{array}{cccc}5593.293 & -4.93 \mathrm{E}-03 & 5593.723 & -4.40 \mathrm{E}-03 \\ 5596.384 & -1.07 \mathrm{E}-03 & 5596.814 & -4.13 \mathrm{E}-03 \\ 5599.475 & -5.63 \mathrm{E}-03 & 5599.905 & -4.99 \mathrm{E}-03 \\ 5602.566 & -4.53 \mathrm{E}-04 & 5602.996 & -4.38 \mathrm{E}-03 \\ 5605.657 & -4.53 \mathrm{E}-03 & 5606.087 & -5.71 \mathrm{E}-03 \\ 5608.748 & 4.25 \mathrm{E}-05 & 5609.178 & -5.25 \mathrm{E}-03 \\ 5611.839 & -4.00 \mathrm{E}-03 & 5612.269 & -5.89 \mathrm{E}-03 \\ 5614.93 & 8.35 \mathrm{E}-04 & 5615.36 & -5.86 \mathrm{E}-03 \\ 5618.021 & -3.89 \mathrm{E}-03 & 5618.451 & -7.15 \mathrm{E}-03 \\ 5621.112 & 1.21 \mathrm{E}-05 & 5621.542 & -5.85 \mathrm{E}-03 \\ 5624.203 & -2.50 \mathrm{E}-03 & 5624.633 & -5.64 \mathrm{E}-03 \\ 5627.294 & 1.90 \mathrm{E}-03 & 5627.724 & -4.41 \mathrm{E}-03 \\ 5630.385 & -2.86 \mathrm{E}-03 & 5630.815 & -5.71 \mathrm{E}-03 \\ 5633.476 & 1.51 \mathrm{E}-03 & 5633.906 & -5.10 \mathrm{E}-03 \\ 5636.567 & -2.42 \mathrm{E}-03 & 5636.997 & -6.03 \mathrm{E}-03 \\ 5639.658 & 1.84 \mathrm{E}-03 & 5640.088 & -5.16 \mathrm{E}-03 \\ 5642.749 & -2.17 \mathrm{E}-03 & 5643.179 & -5.99 \mathrm{E}-03 \\ 5645.84 & 2.01 \mathrm{E}-03 & 5646.27 & -4.98 \mathrm{E}-03 \\ 5648.931 & -2.04 \mathrm{E}-03 & 5649.361 & -5.29 \mathrm{E}-03 \\ 5652.022 & 3.50 \mathrm{E}-03 & 5652.452 & -3.36 \mathrm{E}-03 \\ 5655.113 & -1.88 \mathrm{E}-03 & 5655.543 & -4.50 \mathrm{E}-03 \\ 5658.204 & -3.31 \mathrm{E}-03 & 5658.634 & -4.24 \mathrm{E}-03 \\ 5661.295 & -1.45 \mathrm{E}-04 & 5661.725 & -2.42 \mathrm{E}-03 \\ 5664.386 & -4.49 \mathrm{E}-03 & 5664.816 & -2.20 \mathrm{E}-03 \\ 5667.477 & -1.68 \mathrm{E}-03 & 5667.907 & -7.27 \mathrm{E}-04 \\ 5670.568 & -7.66 \mathrm{E}-03 & 5670.998 & -1.04 \mathrm{E}-03 \\ 5673.659 & -4.36 \mathrm{E}-03 & 5674.089 & 9.98 \mathrm{E}-04 \\ 5676.75 & -9.23 \mathrm{E}-03 & 5677.18 & 1.15 \mathrm{E}-03 \\ 5679.841 & -1.01 \mathrm{E}-02 & 5680.271 & 2.17 \mathrm{E}-03 \\ 5682.932 & -5.46 \mathrm{E}-03 & 5683.362 & 4.25 \mathrm{E}-03 \\ 5686.023 & -8.50 \mathrm{E}-03 & 5686.453 & 5.21 \mathrm{E}-03 \\ 5689.114 & -2.83 \mathrm{E}-03 & 5689.544 & 7.61 \mathrm{E}-03 \\ 5692.3 & -5.82 \mathrm{E}-03 & 5692.73 & 9.47 \mathrm{E}-03 \\ 5692.4 & -6.41 \mathrm{E}-03 & 5692.83 & 9.52 \mathrm{E}-03 \\ 5692.5 & -6.18 \mathrm{E}-03 & 5692.93 & 1.03 \mathrm{E}-02 \\ 5692.6 & -6.04 \mathrm{E}-03 & 5693.03 & 1.05 \mathrm{E}-02 \\ 5692.7 & -6.18 \mathrm{E}-03 & 5693.13 & 1.08 \mathrm{E}-02 \\ 5692.8 & -5.94 \mathrm{E}-03 & 5693.23 & 1.14 \mathrm{E}-02 \\ 5692.9 & -6.07 \mathrm{E}-03 & 5693.33 & 1.13 \mathrm{E}-02 \\ 5693 & -6.53 \mathrm{E}-03 & 5693.43 & 53 \mathrm{E}-02 \\ 5693.1 & -5.68 \mathrm{E}-03 & 5693.53 & \\ 5693.2 & -5.82 \mathrm{E}-03 & 5693.63 & 53 \\ 5693.3 & -5.35 \mathrm{E}-03 & 5693.73 & \\ & & & \end{array}$




$\begin{array}{cccc}5693.4 & -5.04 \mathrm{E}-03 & 5693.83 & 1.11 \mathrm{E}-02 \\ 5693.5 & -4.86 \mathrm{E}-03 & 5693.93 & 1.22 \mathrm{E}-02 \\ 5693.6 & -4.41 \mathrm{E}-03 & 5694.03 & 1.19 \mathrm{E}-02 \\ 5693.7 & -4.07 \mathrm{E}-03 & 5694.13 & 1.18 \mathrm{E}-02 \\ 5693.8 & -4.13 \mathrm{E}-03 & 5694.23 & 1.20 \mathrm{E}-02 \\ 5693.9 & -3.32 \mathrm{E}-03 & 5694.33 & 1.15 \mathrm{E}-02 \\ 5694 & -3.57 \mathrm{E}-03 & 5694.43 & 1.22 \mathrm{E}-02 \\ 5694.1 & -3.47 \mathrm{E}-03 & 5694.53 & 1.26 \mathrm{E}-02 \\ 5694.2 & -3.13 \mathrm{E}-03 & 5694.63 & 1.27 \mathrm{E}-02 \\ 5694.3 & -2.80 \mathrm{E}-03 & 5694.73 & 1.31 \mathrm{E}-02 \\ 5694.4 & -2.57 \mathrm{E}-03 & 5694.83 & 1.28 \mathrm{E}-02 \\ 5694.5 & -2.24 \mathrm{E}-03 & 5694.93 & 1.29 \mathrm{E}-02 \\ 5694.6 & -1.98 \mathrm{E}-03 & 5695.03 & 1.33 \mathrm{E}-02 \\ 5694.7 & -1.81 \mathrm{E}-03 & 5695.13 & 1.37 \mathrm{E}-02 \\ 5694.8 & -1.94 \mathrm{E}-03 & 5695.23 & 1.36 \mathrm{E}-02 \\ 5694.9 & -1.95 \mathrm{E}-03 & 5695.33 & 1.35 \mathrm{E}-02 \\ 5695 & -1.49 \mathrm{E}-03 & 5695.43 & 1.38 \mathrm{E}-02 \\ 5695.1 & -1.40 \mathrm{E}-03 & 5695.53 & 1.33 \mathrm{E}-02 \\ 5695.2 & -1.46 \mathrm{E}-03 & 5695.63 & 1.31 \mathrm{E}-02 \\ 5695.3 & -2.78 \mathrm{E}-04 & 5695.73 & 1.33 \mathrm{E}-02 \\ 5695.4 & -3.94 \mathrm{E}-04 & 5695.83 & 1.37 \mathrm{E}-02 \\ 5695.5 & -2.79 \mathrm{E}-04 & 5695.93 & 1.43 \mathrm{E}-02 \\ 5695.6 & -1.28 \mathrm{E}-04 & 5696.03 & 1.44 \mathrm{E}-02 \\ 5695.7 & -2.11 \mathrm{E}-04 & 5696.13 & 1.45 \mathrm{E}-02 \\ 5695.8 & 5.60 \mathrm{E}-05 & 5696.23 & 1.46 \mathrm{E}-02 \\ 5695.9 & 4.05 \mathrm{E}-04 & 5696.33 & 1.37 \mathrm{E}-02 \\ 5696 & 1.46 \mathrm{E}-04 & 5696.43 & 1.34 \mathrm{E}-02 \\ 5696.1 & 5.05 \mathrm{E}-04 & 5696.53 & 1.32 \mathrm{E}-02 \\ 5696.2 & 5.94 \mathrm{E}-04 & 5696.63 & 1.32 \mathrm{E}-02 \\ 5696.3 & 1.14 \mathrm{E}-03 & 5696.73 & 1.36 \mathrm{E}-02 \\ 5696.4 & 1.32 \mathrm{E}-03 & 5696.83 & 1.36 \mathrm{E}-02 \\ 5696.5 & 2.07 \mathrm{E}-03 & 5696.93 & 1.32 \mathrm{E}-02 \\ 5696.6 & 2.60 \mathrm{E}-03 & 5697.03 & 1.31 \mathrm{E}-02 \\ 5696.7 & 3.04 \mathrm{E}-03 & 5697.13 & 1.37 \mathrm{E}-02 \\ 5696.8 & 2.47 \mathrm{E}-03 & 5697.23 & 1.42 \mathrm{E}-02 \\ 5696.9 & 2.85 \mathrm{E}-03 & 5697.33 & 1.38 \mathrm{E}-02 \\ 5697 & 2.82 \mathrm{E}-03 & 5697.43 & 1.36 \mathrm{E}-02 \\ 5697.1 & 2.67 \mathrm{E}-03 & 5697.53 & 1.43 \mathrm{E}-02 \\ 5697.2 & 2.95 \mathrm{E}-03 & 5697.63 & 1.40 \mathrm{E}-02 \\ 5697.3 & 3.41 \mathrm{E}-03 & 5697.73 & 1.49 \mathrm{E}-02 \\ 5697.4 & 3.96 \mathrm{E}-03 & 5697.83 & \\ 5697.5 & 5.02 \mathrm{E}-03 & 5697.93 & \\ 5697.6 & 4.77 \mathrm{E}-03 & 5698.03 & \\ & & & \\ 5 & & & \\ 5 & & & \\ 5 & & & \end{array}$




\begin{tabular}{|c|c|c|c|}
\hline 5697.7 & $5.28 \mathrm{E}-03$ & 5698.13 & $1.53 \mathrm{E}-02$ \\
\hline 5697.8 & $5.95 \mathrm{E}-03$ & 5698.23 & 1.52E-02 \\
\hline 5697.9 & $6.52 \mathrm{E}-03$ & 5698.33 & 1.56E-02 \\
\hline 5698 & $6.71 \mathrm{E}-03$ & 5698.43 & 1.57E-02 \\
\hline 5698.1 & $6.89 \mathrm{E}-03$ & 5698.53 & $1.61 \mathrm{E}-02$ \\
\hline 5698.2 & 7.53E-03 & 5698.63 & 1.63E-02 \\
\hline 5698.3 & 7.77E-03 & 5698.73 & 1.68E-02 \\
\hline 5698.4 & $7.52 \mathrm{E}-03$ & 5698.83 & 1.73E-02 \\
\hline 5698.5 & 7.52E-03 & 5698.93 & $1.72 \mathrm{E}-02$ \\
\hline 5698.6 & 1.17E-02 & 5699.03 & 1.76E-02 \\
\hline 5698.7 & 1.16E-02 & 5699.13 & 1.79E-02 \\
\hline 5698.8 & $1.02 \mathrm{E}-02$ & 5699.23 & 1.77E-02 \\
\hline 5698.9 & 8.88E-03 & 5699.33 & 1.77E-02 \\
\hline 5699 & 7.19E-03 & 5699.43 & 1.77E-02 \\
\hline 5699.1 & 6.32E-03 & 5699.53 & 1.83E-02 \\
\hline 5699.2 & $4.89 \mathrm{E}-03$ & 5699.63 & 1.86E-02 \\
\hline 5699.3 & $4.00 \mathrm{E}-03$ & 5699.73 & 1.88E-02 \\
\hline 5699.4 & $3.48 \mathrm{E}-03$ & 5699.83 & 1.91E-02 \\
\hline 5699.5 & 2.81E-03 & 5699.93 & 1.93E-02 \\
\hline 5699.6 & 2.33E-03 & 5700.03 & $2.02 \mathrm{E}-02$ \\
\hline 5699.7 & $2.20 \mathrm{E}-03$ & 5700.13 & $2.00 \mathrm{E}-02$ \\
\hline 5699.8 & 1.99E-03 & 5700.23 & $2.02 \mathrm{E}-02$ \\
\hline 5699.9 & $2.37 \mathrm{E}-03$ & 5700.33 & 2.03E-02 \\
\hline 5700 & $2.69 \mathrm{E}-03$ & 5700.43 & 2.07E-02 \\
\hline 5700.1 & $2.96 \mathrm{E}-03$ & 5700.53 & $2.09 \mathrm{E}-02$ \\
\hline 5700.2 & $2.73 \mathrm{E}-03$ & 5700.63 & $2.11 \mathrm{E}-02$ \\
\hline 5700.3 & 2.47E-03 & 5700.73 & 2.10E-02 \\
\hline 5700.4 & 2.67E-03 & 5700.83 & $2.11 \mathrm{E}-02$ \\
\hline 5700.5 & $2.58 \mathrm{E}-03$ & 5700.93 & $2.09 \mathrm{E}-02$ \\
\hline 5700.6 & $2.74 \mathrm{E}-03$ & 5701.03 & $2.13 \mathrm{E}-02$ \\
\hline 5700.7 & $2.40 \mathrm{E}-03$ & 5701.13 & 2.17E-02 \\
\hline 5700.8 & $2.73 \mathrm{E}-03$ & 5701.23 & 2.20E-02 \\
\hline 5700.9 & $3.14 \mathrm{E}-03$ & 5701.33 & 2.25E-02 \\
\hline 5701 & 3.39E-03 & 5701.43 & 2.29E-02 \\
\hline 5701.1 & $3.27 \mathrm{E}-03$ & 5701.53 & 2.30E-02 \\
\hline 5701.2 & $3.44 \mathrm{E}-03$ & 5701.63 & $2.38 \mathrm{E}-02$ \\
\hline 5701.3 & $3.67 \mathrm{E}-03$ & 5701.73 & 2.42E-02 \\
\hline 5701.4 & 4.37E-03 & 5701.83 & 2.38E-02 \\
\hline 5701.5 & $4.01 \mathrm{E}-03$ & 5701.93 & 2.39E-02 \\
\hline 5701.6 & $4.57 \mathrm{E}-03$ & 5702.03 & 2.38E-02 \\
\hline 5701.7 & 4.66E-03 & 5702.13 & $2.36 \mathrm{E}-02$ \\
\hline 5701.8 & 2.97E-03 & 5702.23 & $2.32 \mathrm{E}-02$ \\
\hline 5701.9 & 3.92E-03 & 5702.33 & 2.37E-02 \\
\hline
\end{tabular}




\begin{tabular}{|c|c|c|c|}
\hline 5702 & 3.89E-03 & 5702.43 & $2.40 \mathrm{E}-02$ \\
\hline 5702.1 & $4.28 \mathrm{E}-03$ & 5702.53 & $2.40 \mathrm{E}-02$ \\
\hline 5702.2 & 4.48E-03 & 5702.63 & $2.45 \mathrm{E}-02$ \\
\hline 5702.3 & 4.40E-03 & 5702.73 & $2.52 \mathrm{E}-02$ \\
\hline 5702.4 & 4.35E-03 & 5702.83 & $2.60 \mathrm{E}-\mathrm{C}$ \\
\hline 5702.5 & $4.34 \mathrm{E}-03$ & 5702.93 & $2.62 \mathrm{E}-\mathrm{C}$ \\
\hline 5702.6 & 4.94E-03 & 5703.03 & $2.61 \mathrm{E}-\mathrm{C}$ \\
\hline 5702.7 & 5.65E-03 & 5703.13 & $2.60 \mathrm{E}-\mathrm{C}$ \\
\hline 5702.8 & 5.57E-03 & 5703.23 & $2.64 \mathrm{E}-\mathrm{C}$ \\
\hline 5702.9 & $5.58 \mathrm{E}-03$ & 5703.33 & $2.73 \mathrm{E}-\mathrm{C}$ \\
\hline 5703 & $6.85 \mathrm{E}-03$ & 5703.43 & $2.75 \mathrm{E}-\mathrm{C}$ \\
\hline 5703.1 & $7.18 \mathrm{E}-03$ & 5703.53 & $2.81 \mathrm{E}-\mathrm{C}$ \\
\hline 5703.2 & 7.37E-03 & 5703.63 & $2.84 \mathrm{E}-\mathrm{C}$ \\
\hline 5703.3 & 7.83E-03 & 5703.73 & $2.82 \mathrm{E}-\mathrm{C}$ \\
\hline 5703.4 & 7.87E-03 & 5703.83 & $2.85 \mathrm{E}-($ \\
\hline 5703.5 & 8.32E-03 & 5703.93 & $2.82 \mathrm{E}-($ \\
\hline 5703.6 & 8.65E-03 & 5704.03 & 2.87E-( \\
\hline 5703.7 & 8.46E-03 & 5704.13 & $2.94 \mathrm{E}-($ \\
\hline 5703.8 & $8.63 \mathrm{E}-03$ & 5704.23 & $3.00 \mathrm{E}-($ \\
\hline 5703.9 & 8.77E-03 & 5704.33 & $3.03 \mathrm{E}-($ \\
\hline 5704 & $9.00 \mathrm{E}-03$ & 5704.43 & $3.01 \mathrm{E}-\mathrm{C}$ \\
\hline 5704.1 & 9.32E-03 & 5704.53 & $3.01 \mathrm{E}-0$ \\
\hline 5704.2 & $1.01 \mathrm{E}-02$ & 5704.63 & 3.03E-C \\
\hline 5704.3 & 9.43E-03 & 5704.73 & $3.10 \mathrm{E}-0$ \\
\hline 5704.4 & $9.98 \mathrm{E}-03$ & 5704.83 & $3.14 \mathrm{E}-0$ \\
\hline 5704.5 & 1.04E-02 & 5704.93 & $3.15 \mathrm{E}-0$ \\
\hline 5704.6 & 1.06E-02 & 5705.03 & $3.18 \mathrm{E}-02$ \\
\hline 5704.7 & 1.05E-02 & 5705.13 & $3.24 \mathrm{E}-02$ \\
\hline 5704.8 & 1.14E-02 & 5705.23 & $3.29 \mathrm{E}-02$ \\
\hline 5704.9 & 1.21E-02 & 5705.33 & $3.38 \mathrm{E}-\mathrm{C}$ \\
\hline 5705 & $1.24 \mathrm{E}-02$ & 5705.43 & $3.42 \mathrm{E}-\mathrm{C}$ \\
\hline 5705.1 & 1.33E-02 & 5705.53 & $3.43 \mathrm{E}-\mathrm{C}$ \\
\hline 5705.2 & 1.96E-02 & 5705.63 & $3.53 \mathrm{E}-\mathrm{C}$ \\
\hline 5705.3 & 1.94E-02 & 5705.73 & $3.54 \mathrm{E}-\mathrm{C}$ \\
\hline 5705.4 & 1.92E-02 & 5705.83 & $3.56 \mathrm{E}-($ \\
\hline 5705.5 & $1.85 \mathrm{E}-02$ & 5705.93 & $3.62 \mathrm{E}-\mathrm{C}$ \\
\hline 5705.6 & 1.73E-02 & 5706.03 & $3.62 \mathrm{E}-($ \\
\hline 5705.7 & 1.62E-02 & 5706.13 & $3.68 \mathrm{E}-($ \\
\hline 5705.8 & $1.51 \mathrm{E}-02$ & 5706.23 & $3.69 \mathrm{E}-($ \\
\hline 5705.9 & 1.36E-02 & 5706.33 & $3.78 \mathrm{E}-($ \\
\hline 5706 & $1.29 \mathrm{E}-02$ & 5706.43 & $3.83 \mathrm{E}-($ \\
\hline 5706.1 & $1.24 \mathrm{E}-02$ & 5706.53 & $3.87 \mathrm{E}-($ \\
\hline 5706.2 & 1.14E-02 & 5706.63 & $3.94 \mathrm{E}-\mathrm{-}$ \\
\hline
\end{tabular}




$\begin{array}{cccc}5706.3 & 1.05 \mathrm{E}-02 & 5706.73 & 4.00 \mathrm{E}-02 \\ 5706.4 & 1.03 \mathrm{E}-02 & 5706.83 & 4.03 \mathrm{E}-02 \\ 5706.5 & 9.97 \mathrm{E}-03 & 5706.93 & 4.08 \mathrm{E}-02 \\ 5706.6 & 9.70 \mathrm{E}-03 & 5707.03 & 4.14 \mathrm{E}-02 \\ 5706.7 & 9.69 \mathrm{E}-03 & 5707.13 & 4.19 \mathrm{E}-02 \\ 5706.8 & 9.65 \mathrm{E}-03 & 5707.23 & 4.21 \mathrm{E}-02 \\ 5706.9 & 9.99 \mathrm{E}-03 & 5707.33 & 4.27 \mathrm{E}-02 \\ 5707 & 1.06 \mathrm{E}-02 & 5707.43 & 4.30 \mathrm{E}-02 \\ 5707.1 & 1.07 \mathrm{E}-02 & 5707.53 & 4.32 \mathrm{E}-02 \\ 5707.2 & 1.06 \mathrm{E}-02 & 5707.63 & 4.40 \mathrm{E}-02 \\ 5707.3 & 1.08 \mathrm{E}-02 & 5707.73 & 4.37 \mathrm{E}-02 \\ 5707.4 & 1.16 \mathrm{E}-02 & 5707.83 & 4.46 \mathrm{E}-02 \\ 5707.5 & 1.22 \mathrm{E}-02 & 5707.93 & 4.55 \mathrm{E}-02 \\ 5707.6 & 1.28 \mathrm{E}-02 & 5708.03 & 4.60 \mathrm{E}-02 \\ 5707.7 & 1.32 \mathrm{E}-02 & 5708.13 & 4.65 \mathrm{E}-02 \\ 5707.8 & 1.37 \mathrm{E}-02 & 5708.23 & 4.70 \mathrm{E}-02 \\ 5707.9 & 1.40 \mathrm{E}-02 & 5708.33 & 4.70 \mathrm{E}-02 \\ 5708 & 1.49 \mathrm{E}-02 & 5708.43 & 4.78 \mathrm{E}-02 \\ 5708.1 & 1.52 \mathrm{E}-02 & 5708.53 & 4.81 \mathrm{E}-02 \\ 5708.2 & 1.57 \mathrm{E}-02 & 5708.63 & 4.86 \mathrm{E}-02 \\ 5708.3 & 1.59 \mathrm{E}-02 & 5708.73 & 4.98 \mathrm{E}-02 \\ 5708.4 & 1.61 \mathrm{E}-02 & 5708.83 & 4.97 \mathrm{E}-02 \\ 5708.5 & 1.66 \mathrm{E}-02 & 5708.93 & 5.04 \mathrm{E}-02 \\ 5708.6 & 1.73 \mathrm{E}-02 & 5709.03 & 5.10 \mathrm{E}-02 \\ 5708.7 & 1.72 \mathrm{E}-02 & 5709.13 & 5.23 \mathrm{E}-02 \\ 5708.8 & 1.82 \mathrm{E}-02 & 5709.23 & 5.32 \mathrm{E}-02 \\ 5708.9 & 1.87 \mathrm{E}-02 & 5709.33 & 5.40 \mathrm{E}-02 \\ 5709 & 1.84 \mathrm{E}-02 & 5709.43 & 5.39 \mathrm{E}-02 \\ 5709.1 & 1.88 \mathrm{E}-02 & 5709.53 & 5.45 \mathrm{E}-02 \\ 5709.2 & 2.00 \mathrm{E}-02 & 5709.63 & 5.53 \mathrm{E}-02 \\ 5709.3 & 2.03 \mathrm{E}-02 & 5709.73 & 5.64 \mathrm{E}-02 \\ 5709.4 & 2.09 \mathrm{E}-02 & 5709.83 & 5.64 \mathrm{E}-02 \\ 5709.5 & 2.14 \mathrm{E}-02 & 5709.93 & 5.67 \mathrm{E}-02 \\ 5709.6 & 2.16 \mathrm{E}-02 & 5710.03 & 5.69 \mathrm{E}-02 \\ 5709.7 & 2.30 \mathrm{E}-02 & 5710.13 & 5.73 \mathrm{E}-02 \\ 5709.8 & 2.40 \mathrm{E}-02 & 5710.23 & 5.78 \mathrm{E}-02 \\ 5709.9 & 2.47 \mathrm{E}-02 & 5710.33 & 5.87 \mathrm{E}-02 \\ 5710 & 2.50 \mathrm{E}-02 & 5710.43 & 5.95 \mathrm{E}-02 \\ 5710.1 & 2.55 \mathrm{E}-02 & 5710.53 & 6.06 \mathrm{E}-02 \\ 5710.2 & 2.60 \mathrm{E}-02 & 5710.63 & 6.30 \mathrm{E}-02 \\ 5710.3 & 2.66 \mathrm{E}-02 & 5710.73 & \\ 5710.4 & 2.70 \mathrm{E}-02 & 5710.83 & \\ 5710.5 & 2.73 \mathrm{E}-02 & 5710.93 & \\ & & & \\ 5 & & & \\ 5 & & & \\ 5 & & & \end{array}$




$\begin{array}{cccc}5710.6 & 2.76 \mathrm{E}-02 & 5711.03 & 6.46 \mathrm{E}-02 \\ 5710.7 & 2.65 \mathrm{E}-02 & 5711.13 & 6.55 \mathrm{E}-02 \\ 5710.8 & 2.69 \mathrm{E}-02 & 5711.23 & 6.64 \mathrm{E}-02 \\ 5710.9 & 2.76 \mathrm{E}-02 & 5711.33 & 6.73 \mathrm{E}-02 \\ 5711 & 2.83 \mathrm{E}-02 & 5711.43 & 6.87 \mathrm{E}-02 \\ 5711.1 & 2.89 \mathrm{E}-02 & 5711.53 & 6.98 \mathrm{E}-02 \\ 5711.2 & 2.93 \mathrm{E}-02 & 5711.63 & 7.05 \mathrm{E}-02 \\ 5711.3 & 2.99 \mathrm{E}-02 & 5711.73 & 7.14 \mathrm{E}-02 \\ 5711.4 & 3.07 \mathrm{E}-02 & 5711.83 & 7.26 \mathrm{E}-02 \\ 5711.5 & 3.12 \mathrm{E}-02 & 5711.93 & 7.38 \mathrm{E}-02 \\ 5711.6 & 3.22 \mathrm{E}-02 & 5712.03 & 7.53 \mathrm{E}-02 \\ 5711.7 & 3.30 \mathrm{E}-02 & 5712.13 & 7.63 \mathrm{E}-02 \\ 5711.8 & 3.40 \mathrm{E}-02 & 5712.23 & 7.78 \mathrm{E}-02 \\ 5711.9 & 3.99 \mathrm{E}-02 & 5712.33 & 8.01 \mathrm{E}-02 \\ 5712 & 4.09 \mathrm{E}-02 & 5712.43 & 8.16 \mathrm{E}-02 \\ 5712.1 & 4.09 \mathrm{E}-02 & 5712.53 & 8.28 \mathrm{E}-02 \\ 5712.2 & 4.10 \mathrm{E}-02 & 5712.63 & 8.39 \mathrm{E}-02 \\ 5712.3 & 4.10 \mathrm{E}-02 & 5712.73 & 8.54 \mathrm{E}-02 \\ 5712.4 & 4.08 \mathrm{E}-02 & 5712.83 & 8.67 \mathrm{E}-02 \\ 5712.5 & 3.99 \mathrm{E}-02 & 5712.93 & 8.77 \mathrm{E}-02 \\ 5712.6 & 3.96 \mathrm{E}-02 & 5713.03 & 8.95 \mathrm{E}-02 \\ 5712.7 & 3.95 \mathrm{E}-02 & 5713.13 & 9.04 \mathrm{E}-02 \\ 5712.8 & 3.86 \mathrm{E}-02 & 5713.23 & 9.17 \mathrm{E}-02 \\ 5712.9 & 3.78 \mathrm{E}-02 & 5713.33 & 9.35 \mathrm{E}-02 \\ 5713 & 3.81 \mathrm{E}-02 & 5713.43 & 9.54 \mathrm{E}-02 \\ 5713.1 & 3.76 \mathrm{E}-02 & 5713.53 & 9.69 \mathrm{E}-02 \\ 5713.2 & 3.81 \mathrm{E}-02 & 5713.63 & 9.91 \mathrm{E}-02 \\ 5713.3 & 3.84 \mathrm{E}-02 & 5713.73 & 0.10102102 \\ 5713.4 & 3.87 \mathrm{E}-02 & 5713.83 & 0.10302921 \\ 5713.5 & 3.90 \mathrm{E}-02 & 5713.93 & 0.10481002 \\ 5713.6 & 3.94 \mathrm{E}-02 & 5714.03 & 0.10711212 \\ 5713.7 & 4.02 \mathrm{E}-02 & 5714.13 & 0.10851209 \\ 5713.8 & 4.04 \mathrm{E}-02 & 5714.23 & 0.11019355 \\ 5713.9 & 4.13 \mathrm{E}-02 & 5714.33 & 0.1124995 \\ 5714 & 4.25 \mathrm{E}-02 & 5714.43 & 0.11536963 \\ 5714.1 & 4.37 \mathrm{E}-02 & 5714.53 & 0.11790172 \\ 5714.2 & 4.43 \mathrm{E}-02 & 5714.63 & 0.11995971 \\ 5714.3 & 4.54 \mathrm{E}-02 & 5714.73 & 0.12258743 \\ 5714.4 & 4.69 \mathrm{E}-02 & 5714.83 & 0.12542727 \\ 5714.5 & 4.77 \mathrm{E}-02 & 5714.93 & 0.12832483 \\ 5714.6 & 4.88 \mathrm{E}-02 & 5715.03 & 0.13074241 \\ 5714.7 & 5.01 \mathrm{E}-02 & 5715.13 & 0.13345205 \\ 5714.8 & 5.10 \mathrm{E}-02 & 5715.23 & 0.13689994\end{array}$




$\begin{array}{cccc}5714.9 & 5.23 \mathrm{E}-02 & 5715.33 & 0.14063326 \\ 5715 & 5.32 \mathrm{E}-02 & 5715.43 & 0.14358917 \\ 5715.1 & 5.49 \mathrm{E}-02 & 5715.53 & 0.14674565 \\ 5715.2 & 5.63 \mathrm{E}-02 & 5715.63 & 0.1509049 \\ 5715.3 & 5.77 \mathrm{E}-02 & 5715.73 & 0.15447853 \\ 5715.4 & 5.92 \mathrm{E}-02 & 5715.83 & 0.15865518 \\ 5715.5 & 6.01 \mathrm{E}-02 & 5715.93 & 0.16203755 \\ 5715.6 & 6.19 \mathrm{E}-02 & 5716.03 & 0.16545309 \\ 5715.7 & 6.33 \mathrm{E}-02 & 5716.13 & 0.16980624 \\ 5715.8 & 6.50 \mathrm{E}-02 & 5716.23 & 0.17368534 \\ 5715.9 & 6.67 \mathrm{E}-02 & 5716.33 & 0.17867847 \\ 5716 & 6.89 \mathrm{E}-02 & 5716.43 & 0.18349644 \\ 5716.1 & 7.08 \mathrm{E}-02 & 5716.53 & 0.18881157 \\ 5716.2 & 7.25 \mathrm{E}-02 & 5716.63 & 0.1945208 \\ 5716.3 & 7.56 \mathrm{E}-02 & 5716.73 & 0.19999325 \\ 5716.4 & 7.75 \mathrm{E}-02 & 5716.83 & 0.2056824 \\ 5716.5 & 7.99 \mathrm{E}-02 & 5716.93 & 0.2118607 \\ 5716.6 & 8.14 \mathrm{E}-02 & 5717.03 & 0.21752447 \\ 5716.7 & 8.42 \mathrm{E}-02 & 5717.13 & 0.22350813 \\ 5716.8 & 8.62 \mathrm{E}-02 & 5717.23 & 0.22937478 \\ 5716.9 & 8.83 \mathrm{E}-02 & 5717.33 & 0.2356407 \\ 5717 & 9.14 \mathrm{E}-02 & 5717.43 & 0.24231532 \\ 5717.1 & 9.35 \mathrm{E}-02 & 5717.53 & 0.24858107 \\ 5717.2 & 9.69 \mathrm{E}-02 & 5717.63 & 0.25580555 \\ 5717.3 & 0.10030298 & 5717.73 & 0.26261837 \\ 5717.4 & 0.10364267 & 5717.83 & 0.26954667 \\ 5717.5 & 0.10675285 & 5717.93 & 0.27733763 \\ 5717.6 & 0.10943459 & 5718.03 & 0.28428844 \\ 5717.7 & 0.11312684 & 5718.13 & 0.29236048 \\ 5717.8 & 0.11688631 & 5718.23 & 0.2996543 \\ 5717.9 & 0.12055978 & 5718.33 & 0.30823092 \\ 5718 & 0.12484704 & 5718.43 & 0.31712138 \\ 5718.1 & 0.12853544 & 5718.53 & 0.32460021 \\ 5718.2 & 0.13267313 & 5718.63 & 0.332565 \\ 5718.3 & 0.13769616 & 5718.73 & 0.34175009 \\ 5718.4 & 0.14243271 & 5718.83 & 0.35087721 \\ 5718.5 & 0.1474705 & 5718.93 & 0.36159088 \\ 5718.6 & 0.15718944 & 5719.03 & 0.37239912 \\ 5718.7 & 0.16260984 & 5719.13 & 0.38329251 \\ 5718.8 & 0.16858669 & 5719.23 & 0.39531837 \\ 5718.9 & 0.17326189 & 5719.33 & 0.40637248 \\ 5719 & 0.17790619 & 5719.43 & 0.41883486 \\ 5719.1 & 0.18340108 & 5719.53 & 0.43286113\end{array}$




\begin{tabular}{|c|c|c|c|}
\hline 5719.2 & 0.18810061 & 5719.63 & 0.44632877 \\
\hline 5719.3 & 0.19350521 & 5719.73 & 0.46194378 \\
\hline 5719.4 & 0.19799308 & 5719.83 & 0.47738582 \\
\hline 5719.5 & 0.20226495 & 5719.93 & 0.49351097 \\
\hline 5719.6 & 0.20507078 & 5720.03 & 0.51183102 \\
\hline 5719.7 & 0.20915498 & 5720.13 & 0.52911123 \\
\hline 5719.8 & 0.21287593 & 5720.23 & 0.54825347 \\
\hline 5719.9 & 0.21584675 & 5720.33 & 0.57036598 \\
\hline 5720 & 0.21820971 & 5720.43 & 0.59248305 \\
\hline 5720.1 & 0.22060399 & 5720.53 & 0.61797207 \\
\hline 5720.2 & 0.22242608 & 5720.63 & 0.64243521 \\
\hline 5720.3 & 0.22355855 & 5720.73 & 0.66874339 \\
\hline 5720.4 & 0.2257287 & 5720.83 & 0.69945165 \\
\hline 5720.5 & 0.22802942 & 5720.93 & 0.72912122 \\
\hline 5720.6 & 0.23059546 & 5721.03 & 0.76301141 \\
\hline 5720.7 & 0.23352855 & 5721.13 & 0.79558842 \\
\hline 5720.8 & 0.23762795 & 5721.23 & 0.83038678 \\
\hline 5720.9 & 0.24149388 & 5721.33 & 0.87228569 \\
\hline 5721 & 0.24558876 & 5721.43 & 0.91119179 \\
\hline 5721.1 & 0.25008798 & 5721.53 & 0.95388028 \\
\hline 5721.2 & 0.25603017 & 5721.63 & 1.0026462 \\
\hline 5721.3 & 0.26123541 & 5721.73 & 1.049072 \\
\hline 5721.4 & 0.26768256 & 5721.83 & 1.1014286 \\
\hline 5721.5 & 0.27370505 & 5721.93 & 1.1501986 \\
\hline 5721.6 & 0.28059546 & 5722.03 & 1.2009865 \\
\hline 5721.7 & 0.2882959 & 5722.13 & 1.2588911 \\
\hline 5721.8 & 0.29700187 & 5722.23 & 1.3129801 \\
\hline 5721.9 & 0.30600618 & 5722.33 & 1.3673554 \\
\hline 5722 & 0.31662995 & 5722.43 & 1.4281485 \\
\hline 5722.1 & 0.3266879 & 5722.53 & 1.4829405 \\
\hline 5722.2 & 0.33716859 & 5722.63 & 1.5381421 \\
\hline 5722.3 & 0.3503947 & 5722.73 & 1.5997472 \\
\hline 5722.4 & 0.36288186 & 5722.83 & 1.6549673 \\
\hline 5722.5 & 0.37758429 & 5722.93 & 1.7144477 \\
\hline 5722.6 & 0.39113248 & 5723.03 & 1.7669639 \\
\hline 5722.7 & 0.4058157 & 5723.13 & 1.8172623 \\
\hline 5722.8 & 0.42295544 & 5723.23 & 1.8701634 \\
\hline 5722.9 & 0.43926913 & 5723.33 & 1.9161716 \\
\hline 5723 & 0.45788881 & 5723.43 & 1.9609067 \\
\hline 5723.1 & 0.4786184 & 5723.53 & 2.0073029 \\
\hline 5723.2 & 0.49793723 & 5723.63 & 2.0470608 \\
\hline 5723.3 & 0.52014475 & 5723.73 & 2.0866231 \\
\hline 5723.4 & 0.54115198 & 5723.83 & 2.119953 \\
\hline
\end{tabular}




\begin{tabular}{|c|c|c|c|}
\hline 5723.5 & 0.56269855 & 5723.93 & 2.1511286 \\
\hline 5723.6 & 0.58830702 & 5724.03 & 2.1827782 \\
\hline 5723.7 & 0.61245503 & 5724.13 & 2.207959 \\
\hline 5723.8 & 0.63737609 & 5724.23 & 2.231818 \\
\hline 5723.9 & 0.66688927 & 5724.33 & 2.2550326 \\
\hline 5724 & 0.69356468 & 5724.43 & 2.2732067 \\
\hline 5724.1 & 0.72125513 & 5724.53 & 2.288659 \\
\hline 5724.2 & 0.7529682 & 5724.63 & 2.3039851 \\
\hline 5724.3 & 0.78140478 & 5724.73 & 2.3154518 \\
\hline 5724.4 & 0.81359305 & 5724.83 & 2.3255365 \\
\hline 5724.5 & 0.84302971 & 5724.93 & 2.332531 \\
\hline 5724.6 & 0.87186472 & 5725.03 & 2.3387353 \\
\hline 5724.7 & 0.90467641 & 5725.13 & 2.3430876 \\
\hline 5724.8 & 0.93206138 & 5725.23 & 2.3457371 \\
\hline 5724.9 & 0.95989959 & 5725.33 & 2.345949 \\
\hline 5725 & 0.98942489 & 5725.43 & 2.3442423 \\
\hline 5725.1 & 1.0139675 & 5725.53 & 2.3417872 \\
\hline 5725.2 & 1.0375416 & 5725.63 & 2.3381373 \\
\hline 5725.3 & 1.0662827 & 5725.73 & 2.3350956 \\
\hline 5725.4 & 1.0871041 & 5725.83 & 2.3288454 \\
\hline 5725.5 & 1.1083883 & 5725.93 & 2.3206285 \\
\hline 5725.6 & 1.1252706 & 5726.03 & 2.3120346 \\
\hline 5725.7 & 1.1395509 & 5726.13 & 2.3036674 \\
\hline 5725.8 & 1.1539253 & 5726.23 & 2.292941 \\
\hline 5725.9 & 1.1641354 & 5726.33 & 2.2825444 \\
\hline 5726 & 1.1717292 & 5726.43 & 2.2706189 \\
\hline 5726.1 & 1.1800678 & 5726.53 & 2.256042 \\
\hline 5726.2 & 1.1865659 & 5726.63 & 2.2417931 \\
\hline 5726.3 & 1.191638 & 5726.73 & 2.2257212 \\
\hline 5726.4 & 1.2007666 & 5726.83 & 2.2075041 \\
\hline 5726.5 & 1.2106152 & 5726.93 & 2.189515 \\
\hline 5726.6 & 1.2243313 & 5727.03 & 2.1695619 \\
\hline 5726.7 & 1.2389565 & 5727.13 & 2.1526136 \\
\hline 5726.8 & 1.2545082 & 5727.23 & 2.1355854 \\
\hline 5726.9 & 1.2734266 & 5727.33 & 2.1180579 \\
\hline 5727 & 1.2896352 & 5727.43 & 2.1029801 \\
\hline 5727.1 & 1.3042571 & 5727.53 & 2.0880347 \\
\hline 5727.2 & 1.3185288 & 5727.63 & 2.0719706 \\
\hline 5727.3 & 1.3289769 & 5727.73 & 2.0569965 \\
\hline 5727.4 & 1.3382641 & 5727.83 & 2.0413984 \\
\hline 5727.5 & 1.3511798 & 5727.93 & 2.0230016 \\
\hline 5727.6 & 1.3569784 & 5728.03 & 2.0062485 \\
\hline 5727.7 & 1.3611715 & 5728.13 & 1.9886384 \\
\hline
\end{tabular}




\begin{tabular}{|c|c|c|c|}
\hline 5727.8 & 1.3644855 & 5728.23 & 1.9691464 \\
\hline 5727.9 & 1.3672914 & 5728.33 & 1.9519691 \\
\hline 5728 & 1.369816 & 5728.43 & 1.9319836 \\
\hline 5728.1 & 1.3719176 & 5728.53 & 1.9149693 \\
\hline 5728.2 & 1.3735552 & 5728.63 & 1.8984313 \\
\hline 5728.3 & 1.374588 & 5728.73 & 1.8804566 \\
\hline 5728.4 & 1.3757972 & 5728.83 & 1.8656656 \\
\hline 5728.5 & 1.3758794 & 5728.93 & 1.8503025 \\
\hline 5728.6 & 1.3781164 & 5729.03 & 1.8322227 \\
\hline 5728.7 & 1.3808285 & 5729.13 & 1.8169242 \\
\hline 5728.8 & 1.3842247 & 5729.23 & 1.8016813 \\
\hline 5728.9 & 1.389061 & 5729.33 & 1.7862977 \\
\hline 5729 & 1.3933678 & 5729.43 & 1.7728843 \\
\hline 5729.1 & 1.3989907 & 5729.53 & 1.7585578 \\
\hline 5729.2 & 1.4052179 & 5729.63 & 1.743677 \\
\hline 5729.3 & 1.411238 & 5729.73 & 1.7303081 \\
\hline 5729.4 & 1.4172178 & 5729.83 & 1.717647 \\
\hline 5729.5 & 1.423906 & 5729.93 & 1.7042582 \\
\hline 5729.6 & 1.4294476 & 5730.03 & 1.6909299 \\
\hline 5729.7 & 1.4373096 & 5730.13 & 1.6774895 \\
\hline 5729.8 & 1.443898 & 5730.23 & 1.6662611 \\
\hline 5729.9 & 1.4496134 & 5730.33 & 1.6537787 \\
\hline 5730 & 1.4561733 & 5730.43 & 1.6412418 \\
\hline 5730.1 & 1.4619914 & 5730.53 & 1.6294202 \\
\hline 5730.2 & 1.467719 & 5730.63 & 1.6177899 \\
\hline 5730.3 & 1.4736744 & 5730.73 & 1.605178 \\
\hline 5730.4 & 1.4794035 & 5730.83 & 1.5940655 \\
\hline 5730.5 & 1.4849987 & 5730.93 & 1.5829639 \\
\hline 5730.6 & 1.4905136 & 5731.03 & 1.5705963 \\
\hline 5730.7 & 1.4953379 & 5731.13 & 1.5600089 \\
\hline 5730.8 & 1.4998677 & 5731.23 & 1.5489985 \\
\hline 5730.9 & 1.5051295 & 5731.33 & 1.5368969 \\
\hline 5731 & 1.5086806 & 5731.43 & 1.5262329 \\
\hline 5731.1 & 1.5108861 & 5731.53 & 1.5151921 \\
\hline 5731.2 & 1.5112294 & 5731.63 & 1.5038554 \\
\hline 5731.3 & 1.5118142 & 5731.73 & 1.4939641 \\
\hline 5731.4 & 1.5112747 & 5731.83 & 1.4837995 \\
\hline 5731.5 & 1.5106755 & 5731.93 & 1.4718657 \\
\hline 5731.6 & 1.5116075 & 5732.03 & 1.4610287 \\
\hline 5731.7 & 1.5147695 & 5732.13 & 1.4492274 \\
\hline 5731.8 & 1.5216559 & 5732.23 & 1.4360433 \\
\hline 5731.9 & 1.5312529 & 5732.33 & 1.4244293 \\
\hline 5732 & 1.5492262 & 5732.43 & 1.4157637 \\
\hline
\end{tabular}




\begin{tabular}{|c|c|c|c|}
\hline 5732.1 & 1.5636118 & 5732.53 & 1.4075101 \\
\hline 5732.2 & 1.5787749 & 5732.63 & 1.3996157 \\
\hline 5732.3 & 1.5955624 & 5732.73 & 1.3910523 \\
\hline 5732.4 & 1.610065 & 5732.83 & 1.3839325 \\
\hline 5732.5 & 1.6228714 & 5732.93 & 1.3765745 \\
\hline 5732.6 & 1.6343157 & 5733.03 & 1.3672867 \\
\hline 5732.7 & 1.6419287 & 5733.13 & 1.3587579 \\
\hline 5732.8 & 1.6472939 & 5733.23 & 1.3495425 \\
\hline 5732.9 & 1.6506261 & 5733.33 & 1.3370457 \\
\hline 5733 & 1.6503383 & 5733.43 & 1.3264526 \\
\hline 5733.1 & 1.6504546 & 5733.53 & 1.3163579 \\
\hline 5733.2 & 1.6518459 & 5733.63 & 1.3063121 \\
\hline 5733.3 & 1.6554623 & 5733.73 & 1.2992276 \\
\hline 5733.4 & 1.66217 & 5733.83 & 1.2943679 \\
\hline 5733.5 & 1.6730005 & 5733.93 & 1.2900835 \\
\hline 5733.6 & 1.6852114 & 5734.03 & 1.2869164 \\
\hline 5733.7 & 1.6987121 & 5734.13 & 1.2832662 \\
\hline 5733.8 & 1.7141134 & 5734.23 & 1.2787884 \\
\hline 5733.9 & 1.726318 & 5734.33 & 1.274352 \\
\hline 5734 & 1.737774 & 5734.43 & 1.2691406 \\
\hline 5734.1 & 1.7509995 & 5734.53 & 1.2625434 \\
\hline 5734.2 & 1.7649698 & 5734.63 & 1.2560308 \\
\hline 5734.3 & 1.7770778 & 5734.73 & 1.2492472 \\
\hline 5734.4 & 1.7879419 & 5734.83 & 1.2429016 \\
\hline 5734.5 & 1.7957467 & 5734.93 & 1.2365557 \\
\hline 5734.6 & 1.801557 & 5735.03 & 1.2306071 \\
\hline 5734.7 & 1.8057017 & 5735.13 & 1.2238337 \\
\hline 5734.8 & 1.8075981 & 5735.23 & 1.2183559 \\
\hline 5734.9 & 1.8090273 & 5735.33 & 1.2129945 \\
\hline 5735 & 1.8119038 & 5735.43 & 1.206635 \\
\hline 5735.1 & 1.816418 & 5735.53 & 1.2005646 \\
\hline 5735.2 & 1.8216257 & 5735.63 & 1.1944681 \\
\hline 5735.3 & 1.8291917 & 5735.73 & 1.1880895 \\
\hline 5735.4 & 1.8351078 & 5735.83 & 1.1830312 \\
\hline 5735.5 & 1.840018 & 5735.93 & 1.1777274 \\
\hline 5735.6 & 1.8451372 & 5736.03 & 1.1731793 \\
\hline 5735.7 & 1.8490813 & 5736.13 & 1.1692067 \\
\hline 5735.8 & 1.8512556 & 5736.23 & 1.165143 \\
\hline 5735.9 & 1.8525327 & 5736.33 & 1.1599142 \\
\hline 5736 & 1.8531935 & 5736.43 & 1.156186 \\
\hline 5736.1 & 1.8521338 & 5736.53 & 1.1523918 \\
\hline 5736.2 & 1.8500169 & 5736.63 & 1.1476597 \\
\hline 5736.3 & 1.8461685 & 5736.73 & 1.1436577 \\
\hline
\end{tabular}




\begin{tabular}{|c|c|c|c|}
\hline 5736.4 & 1.8413101 & 5736.83 & 1.1393723 \\
\hline 5736.5 & 1.8379726 & 5736.93 & 1.1347695 \\
\hline 5736.6 & 1.8299616 & 5737.03 & 1.1307209 \\
\hline 5736.7 & 1.8215748 & 5737.13 & 1.1269159 \\
\hline 5736.8 & 1.8101692 & 5737.23 & 1.1224285 \\
\hline 5736.9 & 1.7990813 & 5737.33 & 1.1187423 \\
\hline 5737 & 1.7860116 & 5737.43 & 1.1147933 \\
\hline 5737.1 & 1.770612 & 5737.53 & 1.1101447 \\
\hline 5737.2 & 1.756205 & 5737.63 & 1.106393 \\
\hline 5737.3 & 1.7405561 & 5737.73 & 1.1023006 \\
\hline 5737.4 & 1.7225179 & 5737.83 & 1.0983425 \\
\hline 5737.5 & 1.7067165 & 5737.93 & 1.0949237 \\
\hline 5737.6 & 1.6903726 & 5738.03 & 1.0912458 \\
\hline 5737.7 & 1.6703616 & 5738.13 & 1.0872597 \\
\hline 5737.8 & 1.6528836 & 5738.23 & 1.0834683 \\
\hline 5737.9 & 1.6344129 & 5738.33 & 1.0795141 \\
\hline 5738 & 1.6141836 & 5738.43 & 1.0750584 \\
\hline 5738.1 & 1.5961653 & 5738.53 & 1.0711889 \\
\hline 5738.2 & 1.5773508 & 5738.63 & 1.0663615 \\
\hline 5738.3 & 1.5557289 & 5738.73 & 1.0616233 \\
\hline 5738.4 & 1.5352999 & 5738.83 & 1.0567292 \\
\hline 5738.5 & 1.5152407 & 5738.93 & 1.0519207 \\
\hline 5738.6 & 1.4934106 & 5739.03 & 1.0468697 \\
\hline 5738.7 & 1.4801623 & 5739.13 & 1.044643 \\
\hline 5738.8 & 1.4655717 & 5739.23 & 1.0422795 \\
\hline 5738.9 & 1.4514159 & 5739.33 & 1.0407089 \\
\hline 5739 & 1.4418897 & 5739.43 & 1.0409103 \\
\hline 5739.1 & 1.4357955 & 5739.53 & 1.0420969 \\
\hline 5739.2 & 1.432229 & 5739.63 & 1.0443218 \\
\hline 5739.3 & 1.4311068 & 5739.73 & 1.0451749 \\
\hline 5739.4 & 1.4303218 & 5739.83 & 1.0462003 \\
\hline 5739.5 & 1.4284474 & 5739.93 & 1.0458983 \\
\hline 5739.6 & 1.4252269 & 5740.03 & 1.0443308 \\
\hline 5739.7 & 1.4203204 & 5740.13 & 1.0418484 \\
\hline 5739.8 & 1.4113402 & 5740.23 & 1.0386308 \\
\hline 5739.9 & 1.4028866 & 5740.33 & 1.0362517 \\
\hline 5740 & 1.3941878 & 5740.43 & 1.0334746 \\
\hline 5740.1 & 1.3836246 & 5740.53 & 1.0304337 \\
\hline 5740.2 & 1.3727321 & 5740.63 & 1.0272773 \\
\hline 5740.3 & 1.3597895 & 5740.73 & 1.0243124 \\
\hline 5740.4 & 1.3471641 & 5740.83 & 1.0218065 \\
\hline 5740.5 & 1.3321584 & 5740.93 & 1.0187432 \\
\hline 5740.6 & 1.3192483 & 5741.03 & 1.0157505 \\
\hline
\end{tabular}




\begin{tabular}{|c|c|c|c|}
\hline 5740.7 & 1.3079097 & 5741.13 & 1.0128355 \\
\hline 5740.8 & 1.2970937 & 5741.23 & 1.0102844 \\
\hline 5740.9 & 1.2893601 & 5741.33 & 1.0077302 \\
\hline 5741 & 1.2826391 & 5741.43 & 1.0052058 \\
\hline 5741.1 & 1.2749915 & 5741.53 & 1.0027512 \\
\hline 5741.2 & 1.2681427 & 5741.63 & 1.0018122 \\
\hline 5741.3 & 1.2614348 & 5741.73 & 1.0007343 \\
\hline 5741.4 & 1.2535129 & 5741.83 & 0.99913893 \\
\hline 5741.5 & 1.2462469 & 5741.93 & 0.99775312 \\
\hline 5741.6 & 1.2388325 & 5742.03 & 0.99655567 \\
\hline 5741.7 & 1.2311383 & 5742.13 & 0.99532807 \\
\hline 5741.8 & 1.2246634 & 5742.23 & 0.99391955 \\
\hline 5741.9 & 1.2177965 & 5742.33 & 0.99271948 \\
\hline 5742 & 1.2110604 & 5742.43 & 0.99102029 \\
\hline 5742.1 & 1.2056818 & 5742.53 & 0.9895909 \\
\hline 5742.2 & 1.2000627 & 5742.63 & 0.98872507 \\
\hline 5742.3 & 1.1937549 & 5742.73 & 0.98778118 \\
\hline 5742.4 & 1.1884152 & 5742.83 & 0.98730239 \\
\hline 5742.5 & 1.1830789 & 5742.93 & 0.9861293 \\
\hline 5742.6 & 1.1778595 & 5743.03 & 0.98469974 \\
\hline 5742.7 & 1.1717964 & 5743.13 & 0.9833873 \\
\hline 5742.8 & 1.1676581 & 5743.23 & 0.98281868 \\
\hline 5742.9 & 1.1631039 & 5743.33 & 0.9817286 \\
\hline 5743 & 1.1572061 & 5743.43 & 0.98066279 \\
\hline 5743.1 & 1.1536975 & 5743.53 & 0.97913224 \\
\hline 5743.2 & 1.1541846 & 5743.63 & 0.9778459 \\
\hline 5743.3 & 1.149371 & 5743.73 & 0.9772428 \\
\hline 5743.4 & 1.1450229 & 5743.83 & 0.9767357 \\
\hline 5743.5 & 1.1408862 & 5743.93 & 0.97566685 \\
\hline 5743.6 & 1.1350109 & 5744.03 & 0.9747742 \\
\hline 5743.7 & 1.1307892 & 5744.13 & 0.97374436 \\
\hline 5743.8 & 1.1268294 & 5744.23 & 0.97265959 \\
\hline 5743.9 & 1.1219913 & 5744.33 & 0.97156409 \\
\hline 5744 & 1.1184433 & 5744.43 & 0.9707792 \\
\hline 5744.1 & 1.1143102 & 5744.53 & 0.97033646 \\
\hline 5744.2 & 1.1101946 & 5744.63 & 0.96980467 \\
\hline 5744.3 & 1.1054197 & 5744.73 & 0.9680350 \\
\hline 5744.4 & 1.1016547 & 5744.83 & 0.9675351 \\
\hline 5744.5 & 1.0975125 & 5744.93 & 0.9670117 \\
\hline 5744.6 & 1.092971 & 5745.03 & 0.9659114 \\
\hline 5744.7 & 1.089007 & 5745.13 & 0.9655398 \\
\hline 5744.8 & 1.0857299 & 5745.23 & 0.9655191 \\
\hline 5744.9 & 1.0819904 & 5745.33 & 0.9640471 \\
\hline
\end{tabular}




$\begin{array}{cccc}5745 & 1.0786353 & 5745.43 & 0.96285506 \\ 5745.1 & 1.0749707 & 5745.53 & 0.96267041 \\ 5745.2 & 1.0709428 & 5745.63 & 0.96208602 \\ 5745.3 & 1.0672602 & 5745.73 & 0.96143099 \\ 5745.4 & 1.0644856 & 5745.83 & 0.96125803 \\ 5745.5 & 1.0615172 & 5745.93 & 0.96182194 \\ 5745.6 & 1.058516 & 5746.03 & 0.96182986 \\ 5745.7 & 1.0556759 & 5746.13 & 0.96105081 \\ 5745.8 & 1.0519838 & 5746.23 & 0.96025926 \\ 5745.9 & 1.0492301 & 5746.33 & 0.95987966 \\ 5746 & 1.046152 & 5746.43 & 0.95931252 \\ 5746.1 & 1.0431299 & 5746.53 & 0.95871152 \\ 5746.2 & 1.0403097 & 5746.63 & 0.95795219 \\ 5746.3 & 1.0373023 & 5746.73 & 0.95708023 \\ 5746.4 & 1.0348089 & 5746.83 & 0.95706718 \\ 5746.5 & 1.0299788 & 5746.93 & 0.95718091 \\ 5746.6 & 1.0280792 & 5747.03 & 0.95705026 \\ 5746.7 & 1.0253614 & 5747.13 & 0.95745847 \\ 5746.8 & 1.022665 & 5747.23 & 0.9575152 \\ 5746.9 & 1.0197643 & 5747.33 & 0.95676688 \\ 5747 & 1.0177841 & 5747.43 & 0.95624383 \\ 5747.1 & 1.016093 & 5747.53 & 0.95629081 \\ 5747.2 & 1.013714 & 5747.63 & 0.95551354 \\ 5747.3 & 1.0111843 & 5747.73 & 0.95562013 \\ 5747.4 & 1.0086756 & 5747.83 & 0.95621253 \\ 5747.5 & 1.0064854 & 5747.93 & 0.95596561 \\ 5747.6 & 1.0047228 & 5748.03 & 0.9560886 \\ 5747.7 & 1.0032542 & 5748.13 & 0.95612379 \\ 5747.8 & 1.0051249 & 5748.23 & 0.95622822 \\ 5747.9 & 1.0039328 & 5748.33 & 0.95655522 \\ 5748 & 1.0020246 & 5748.43 & 0.95675094 \\ 5748.1 & 0.99945806 & 5748.53 & 0.95694194 \\ 5748.2 & 0.99646872 & 5748.63 & 0.95685595 \\ 5748.3 & 0.99302359 & 5748.73 & 0.95699047 \\ 5748.4 & 0.990413 & 5748.83 & 0.95726906 \\ 5748.5 & 0.98673015 & 5748.93 & 0.95730382 \\ 5748.6 & 0.98346184 & 5749.03 & 0.95702533 \\ 5748.7 & 0.97957567 & 5749.13 & 0.95724351 \\ 5748.8 & 0.9766961 & 5749.23 & 0.95735013 \\ 5748.9 & 0.97431392 & 5749.33 & 0.95761189 \\ 5749 & 0.97153702 & 5749.43 & 0.95806054 \\ 5749.1 & 0.96950694 & 5749.53 & 0.9583916 \\ 5749.2 & 0.96768887 & 5749.63 & 0.95941662\end{array}$




$\begin{array}{cccc}5749.3 & 0.96610807 & 5749.73 & 0.96032105 \\ 5749.4 & 0.96415824 & 5749.83 & 0.95980101 \\ 5749.5 & 0.96264945 & 5749.93 & 0.96006475 \\ 5749.6 & 0.96070221 & 5750.03 & 0.96043592 \\ 5749.7 & 0.95910433 & 5750.13 & 0.96056609 \\ 5749.8 & 0.95743824 & 5750.23 & 0.9615373 \\ 5749.9 & 0.95657899 & 5750.33 & 0.96211673 \\ 5750 & 0.95678457 & 5750.43 & 0.96268902 \\ 5750.1 & 0.95554327 & 5750.53 & 0.96281179 \\ 5750.2 & 0.95413736 & 5750.63 & 0.96302224 \\ 5750.3 & 0.95273569 & 5750.73 & 0.96468943 \\ 5750.4 & 0.95166775 & 5750.83 & 0.96528153 \\ 5750.5 & 0.94990921 & 5750.93 & 0.96577628 \\ 5750.6 & 0.94870174 & 5751.03 & 0.96658021 \\ 5750.7 & 0.94712667 & 5751.13 & 0.96685538 \\ 5750.8 & 0.946141 & 5751.23 & 0.96780582 \\ 5750.9 & 0.94515921 & 5751.33 & 0.96875994 \\ 5751 & 0.9439128 & 5751.43 & 0.96924421 \\ 5751.1 & 0.94258756 & 5751.53 & 0.96942016 \\ 5751.2 & 0.94195479 & 5751.63 & 0.9693744 \\ 5751.3 & 0.94099914 & 5751.73 & 0.97101168 \\ 5751.4 & 0.94020426 & 5751.83 & 0.97192151 \\ 5751.5 & 0.93953554 & 5751.93 & 0.97294282 \\ 5751.6 & 0.93831708 & 5752.03 & 0.97405439 \\ 5751.7 & 0.9375075 & 5752.13 & 0.97515235 \\ 5751.8 & 0.93680657 & 5752.23 & 0.97576558 \\ 5751.9 & 0.93572508 & 5752.33 & 0.97697144 \\ 5752 & 0.93499945 & 5752.43 & 0.97767618 \\ 5752.3 & 0.93561382 & 5752.73 & 0.98061086 \\ 5752.602 & 0.93266465 & 5753.032 & 0.98226605 \\ 5752.904 & 0.93002057 & 5753.334 & 0.98413803 \\ 5753.206 & 0.92750683 & 5753.636 & 0.9870456 \\ 5753.508 & 0.92334513 & 5753.938 & 0.98980004 \\ 5753.81 & 0.9206031 & 5754.24 & 0.99219984 \\ 5754.112 & 0.91858433 & 5754.542 & 0.99443606 \\ 5754.414 & 0.91738379 & 5754.844 & 0.99797976 \\ 5754.716 & 0.9206115 & 5755.146 & 1.0001803 \\ 5755.018 & 0.91925393 & 5755.448 & 1.0032157 \\ 5755.32 & 0.91765649 & 5755.75 & 1.0062751 \\ 5755.622 & 0.91643635 & 5756.052 & 1.0088093 \\ 5755.924 & 0.91689338 & 5756.354 & 1.010581 \\ 5756.226 & 0.91606874 & 5756.656 & 57632 \\ 5756.528 & 0.91482448 & 5756.958 & \\ & & & \\ 5 & & & \\ & & & \end{array}$




\begin{tabular}{|c|c|c|c|}
\hline 5756.83 & 0.91645727 & 5757.26 & 1.0214744 \\
\hline 5757.132 & 0.91621383 & 5757.562 & 1.0249395 \\
\hline 5757.434 & 0.91619556 & 5757.864 & 1.0279314 \\
\hline 5757.736 & 0.91686033 & 5758.166 & 1.0312227 \\
\hline 5758.038 & 0.91798013 & 5758.468 & 1.0336121 \\
\hline 5758.34 & 0.91836301 & 5758.77 & 1.0362808 \\
\hline 5758.642 & 0.91940447 & 5759.072 & 1.0393541 \\
\hline 5758.944 & 0.92048877 & 5759.374 & 1.0421135 \\
\hline 5759.246 & 0.92262776 & 5759.676 & 1.0448906 \\
\hline 5759.548 & 0.92379541 & 5759.978 & 1.0473191 \\
\hline 5759.85 & 0.92433974 & 5760.28 & 1.0499711 \\
\hline 5760.152 & 0.92576466 & 5760.582 & 1.0521983 \\
\hline 5760.454 & 0.92730956 & 5760.884 & 1.0537557 \\
\hline 5760.756 & 0.92796685 & 5761.186 & 1.0567191 \\
\hline 5761.058 & 0.92851991 & 5761.488 & 1.0584756 \\
\hline 5761.36 & 0.93148151 & 5761.79 & 1.062299 \\
\hline 5761.662 & 0.93137643 & 5762.092 & 1.0646762 \\
\hline 5761.964 & 0.93123705 & 5762.394 & 1.0659589 \\
\hline 5762.266 & 0.93092538 & 5762.696 & 1.0675428 \\
\hline 5762.568 & 0.93031919 & 5762.998 & 1.0674715 \\
\hline 5762.87 & 0.93037968 & 5763.3 & 1.0682968 \\
\hline 5763.172 & 0.93096775 & 5763.602 & 1.069035 \\
\hline 5763.474 & 0.93197641 & 5763.904 & 1.0701151 \\
\hline 5763.776 & 0.93745058 & 5764.206 & 1.0712049 \\
\hline 5764.078 & 0.93924127 & 5764.508 & 1.0717746 \\
\hline 5764.38 & 0.94075243 & 5764.81 & 1.0728138 \\
\hline 5764.682 & 0.94025694 & 5765.112 & 1.0730602 \\
\hline 5764.984 & 0.94191063 & 5765.414 & 1.0735282 \\
\hline 5765.286 & 0.94337405 & 5765.716 & 1.0737967 \\
\hline 5765.588 & 0.94469403 & 5766.018 & 1.073515 \\
\hline 5765.89 & 0.94671653 & 5766.32 & 1.0737411 \\
\hline 5766.192 & 0.95074718 & 5766.622 & 1.0744495 \\
\hline 5766.494 & 0.95292818 & 5766.924 & 1.0742091 \\
\hline 5766.796 & 0.95453394 & 5767.226 & 1.0746072 \\
\hline 5767.098 & 0.95696876 & 5767.528 & 1.0745881 \\
\hline 5767.4 & 0.95892625 & 5767.83 & 1.0745539 \\
\hline 5767.702 & 0.96124455 & 5768.132 & 1.074401 \\
\hline 5768.004 & 0.96365978 & 5768.434 & 1.0740882 \\
\hline 5768.306 & 0.96960159 & 5768.736 & 1.0742356 \\
\hline 5768.608 & 0.9714201 & 5769.038 & 1.0735498 \\
\hline 5768.91 & 0.97367257 & 5769.34 & 1.07286 \\
\hline 5769.212 & 0.97574855 & 5769.642 & 1.0720634 \\
\hline 5769.514 & 0.97789656 & 5769.944 & 1.0719967 \\
\hline
\end{tabular}




\begin{tabular}{|c|c|c|c|}
\hline 5769.816 & 0.9794201 & 5770.246 & 1.0716471 \\
\hline 5770.118 & 0.98093079 & 5770.548 & 1.0703854 \\
\hline 5770.42 & 0.98231705 & 5770.85 & 1.06939 \\
\hline 5770.722 & 0.98608925 & 5771.152 & 1.0698614 \\
\hline 5771.024 & 0.98779075 & 5771.454 & 1.0690829 \\
\hline 5771.326 & 0.98975429 & 5771.756 & 1.0681266 \\
\hline 5771.628 & 0.99235942 & 5772.058 & 1.0663204 \\
\hline 5771.93 & 0.99335444 & 5772.36 & 1.0652602 \\
\hline 5772.232 & 0.99499936 & 5772.662 & 1.0639976 \\
\hline 5772.534 & 0.99662083 & 5772.964 & 1.063452 \\
\hline 5772.836 & 1.0013206 & 5773.266 & 1.0631818 \\
\hline 5773.138 & 1.0026725 & 5773.568 & 1.0614468 \\
\hline 5773.44 & 1.0046589 & 5773.87 & 1.0591081 \\
\hline 5773.742 & 1.0055464 & 5774.172 & 1.05805 \\
\hline 5774.044 & 1.0064003 & 5774.474 & 1.0565598 \\
\hline 5774.346 & 1.0077571 & 5774.776 & 1.0541823 \\
\hline 5774.648 & 1.0089339 & 5775.078 & 1.0522007 \\
\hline 5774.95 & 1.0095926 & 5775.38 & 1.0504615 \\
\hline 5775.252 & 1.0134733 & 5775.682 & 1.0490159 \\
\hline 5775.554 & 1.0140689 & 5775.984 & 1.0464472 \\
\hline 5775.856 & 1.0147275 & 5776.286 & 1.0446248 \\
\hline 5776.158 & 1.0149866 & 5776.588 & 1.0423317 \\
\hline 5776.46 & 1.0164725 & 5776.89 & 1.0391365 \\
\hline 5776.762 & 1.0169341 & 5777.192 & 1.0359996 \\
\hline 5777.064 & 1.0182185 & 5777.494 & 1.0339844 \\
\hline 5777.366 & 1.019219 & 5777.796 & 1.0322815 \\
\hline 5777.668 & 1.0242675 & 5778.098 & 1.0307696 \\
\hline 5777.97 & 1.0253508 & 5778.4 & 1.0284029 \\
\hline 5778.272 & 1.0258203 & 5778.702 & 1.0264983 \\
\hline 5778.574 & 1.0264022 & 5779.004 & 1.0240442 \\
\hline 5778.876 & 1.0267139 & 5779.306 & 1.0223987 \\
\hline 5779.178 & 1.0266425 & 5779.608 & 1.0203047 \\
\hline 5779.48 & 1.0276804 & 5779.91 & 1.0186629 \\
\hline 5779.782 & 1.02983 & 5780.212 & 1.017047 \\
\hline 5780.084 & 1.0302952 & 5780.514 & 1.0151137 \\
\hline 5780.386 & 1.0308651 & 5780.816 & 1.0137754 \\
\hline 5780.688 & 1.0309867 & 5781.118 & 1.012038 \\
\hline 5780.99 & 1.0310191 & 5781.42 & 1.0099939 \\
\hline 5781.292 & 1.0310603 & 5781.722 & 1.0082482 \\
\hline 5781.594 & 1.0311166 & 5782.024 & 1.005873 \\
\hline 5781.896 & 1.0309141 & 5782.326 & 1.0048487 \\
\hline 5782.198 & 1.0356312 & 5782.628 & 1.0037675 \\
\hline 5782.5 & 1.0353439 & 5782.93 & 1.0029654 \\
\hline
\end{tabular}




$\begin{array}{cccc}5782.802 & 1.0351726 & 5783.232 & 1.0017517 \\ 5783.104 & 1.0357219 & 5783.534 & 1.0002712 \\ 5783.406 & 1.0357475 & 5783.836 & 0.99881239 \\ 5783.708 & 1.0352166 & 5784.138 & 0.99846832 \\ 5784.01 & 1.034348 & 5784.44 & 0.99726619 \\ 5784.312 & 1.0361682 & 5784.742 & 0.99647904 \\ 5784.614 & 1.0345077 & 5785.044 & 0.9947798 \\ 5784.916 & 1.0320346 & 5785.346 & 0.99289933 \\ 5785.218 & 1.0297644 & 5785.648 & 0.99238377 \\ 5785.52 & 1.0268062 & 5785.95 & 0.99145205 \\ 5785.822 & 1.0252122 & 5786.252 & 0.99029494 \\ 5786.124 & 1.0236341 & 5786.554 & 0.98934598 \\ 5786.426 & 1.0221422 & 5786.856 & 0.98814579 \\ 5786.728 & 1.0237271 & 5787.158 & 0.9884134 \\ 5787.03 & 1.0226471 & 5787.46 & 0.98692065 \\ 5787.332 & 1.0216306 & 5787.762 & 0.98603965 \\ 5787.634 & 1.0204797 & 5788.064 & 0.98585052 \\ 5787.936 & 1.0189122 & 5788.366 & 0.98468733 \\ 5788.238 & 1.0175712 & 5788.668 & 0.98417023 \\ 5788.54 & 1.016458 & 5788.97 & 0.98359949 \\ 5788.842 & 1.0157525 & 5789.272 & 0.98335432 \\ 5789.144 & 1.0166648 & 5789.574 & 0.98330042 \\ 5789.446 & 1.0158856 & 5789.876 & 0.98305571 \\ 5789.748 & 1.0153686 & 5790.178 & 0.98360256 \\ 5790.05 & 1.0144414 & 5790.48 & 0.98223193 \\ 5790.352 & 1.0139468 & 5790.782 & 0.98214073 \\ 5790.654 & 1.0130306 & 5791.084 & 0.98206558 \\ 5790.956 & 1.0124204 & 5791.386 & 0.98074865 \\ 5791.258 & 1.0156629 & 5791.688 & 0.98112079 \\ 5791.56 & 1.0143391 & 5791.99 & 0.98038154 \\ 5791.862 & 1.0136824 & 5792.292 & 0.98016401 \\ 5795.5 & 0.99968414 & 5795.93 & 0.97784566 \\ 5798.616 & 0.99442472 & 5799.046 & 0.97934106 \\ 5801.732 & 0.97852455 & 5802.162 & 0.98037635 \\ 5804.848 & 0.9733289 & 5805.278 & 0.98836437 \\ 5807.964 & 0.96083706 & 5808.394 & 0.99679166 \\ 5811.08 & 0.96233134 & 5811.51 & 1.0078711 \\ 5814.196 & 0.95635948 & 5814.626 & 1.0148451 \\ 5817.312 & 0.95755386 & 5817.742 & 1.0187873 \\ 5820.428 & 0.96983264 & 5820.858 & 1.020852 \\ 5823.544 & 0.97305282 & 5823.974 & 1.0188552 \\ 5826.66 & 0.98115641 & 5827.09 & 1.0148323 \\ 5829.776 & 0.99079761 & 5830.206 & \\ & & & \\ 5 & & & \\ 5 & & & \\ 5 & & & \end{array}$




\begin{tabular}{|c|c|c|c|}
\hline 5832.892 & 1.003727 & 5833.322 & 1.0136509 \\
\hline 5836.008 & 1.005839 & 5836.438 & 1.0095218 \\
\hline 5839.124 & 1.0094779 & 5839.554 & 1.0082423 \\
\hline 5842.24 & 1.0167378 & 5842.67 & 1.009508 \\
\hline 5845.356 & 1.0156112 & 5845.786 & 1.0134694 \\
\hline 5848.472 & 1.0198452 & 5848.902 & 1.0159959 \\
\hline 5851.588 & 1.0288628 & 5852.018 & 1.015857 \\
\hline 5854.704 & 1.029862 & 5855.134 & 1.0108 \\
\hline 5857.82 & 1.0295713 & 5858.25 & 1.0041267 \\
\hline 5860.936 & 1.0291829 & 5861.366 & 0.9994949 \\
\hline 5864.052 & 1.0148957 & 5864.482 & 0.99342527 \\
\hline 5867.168 & 1.0086228 & 5867.598 & 0.98924863 \\
\hline 5870.284 & 1.0062567 & 5870.714 & 0.98746377 \\
\hline 5873.4 & 1.0090325 & 5873.83 & 0.98694377 \\
\hline 5876.516 & 1.0017824 & 5876.946 & 0.98596939 \\
\hline 5879.632 & 0.99801548 & 5880.062 & 0.98677484 \\
\hline 5882.748 & 0.99949547 & 5883.178 & 0.99110811 \\
\hline 5885.864 & 0.9933786 & 5886.294 & 0.99421973 \\
\hline 5888.98 & 0.98893866 & 5889.41 & 0.99759848 \\
\hline 5892.096 & 0.98830816 & 5892.526 & 1.0006484 \\
\hline 5895.212 & 0.99335822 & 5895.642 & 1.0052816 \\
\hline 5898.328 & 0.99195915 & 5898.758 & 1.0059779 \\
\hline 5901.444 & 0.99038948 & 5901.874 & 1.0059674 \\
\hline 5904.56 & 0.99138022 & 5904.99 & 1.0057395 \\
\hline 5907.676 & 0.99713001 & 5908.106 & 1.0049716 \\
\hline 5910.792 & 0.99415092 & 5911.222 & 1.0017997 \\
\hline 5913.908 & 0.99571552 & 5914.338 & 1.0000721 \\
\hline 5917.024 & 0.9947167 & 5917.454 & 0.99754869 \\
\hline 5920.14 & 1.0026925 & 5920.57 & 0.99720375 \\
\hline 5923.256 & 1.0002294 & 5923.686 & 0.99402097 \\
\hline 5926.372 & 1.0014014 & 5926.802 & 0.9935891 \\
\hline 5929.488 & 1.0012109 & 5929.918 & 0.99230438 \\
\hline 5932.604 & 1.0004008 & 5933.034 & 0.99127842 \\
\hline 5935.72 & 0.99883111 & 5936.15 & 0.99129394 \\
\hline 5938.836 & 1.0058704 & 5939.266 & 0.99301589 \\
\hline 5941.952 & 1.0001703 & 5942.382 & 0.99311025 \\
\hline 5945.068 & 1.0003467 & 5945.498 & 0.99387507 \\
\hline 5948.184 & 0.99904011 & 5948.614 & 0.99512372 \\
\hline 5951.3 & 1.0045825 & 5951.73 & 0.99677551 \\
\hline 5954.416 & 1.002196 & 5954.846 & 0.9969338 \\
\hline 5957.532 & 0.99975536 & 5957.962 & 0.99615166 \\
\hline 5960.648 & 0.99981041 & 5961.078 & 0.99723239 \\
\hline 5963.764 & 1.0045994 & 5964.194 & 0.99887059 \\
\hline
\end{tabular}




$\begin{array}{cccc}5966.88 & 0.99959992 & 5967.31 & 0.99937707 \\ 5969.996 & 1.0004955 & 5970.426 & 0.99997143 \\ 5973.112 & 0.99963558 & 5973.542 & 1.0014451 \\ 5976.3 & 1.0048619 & 5976.73 & 1.0035194 \\ 5976.4 & 1.0048851 & 5976.83 & 1.0035162 \\ 5976.5 & 1.0048589 & 5976.93 & 1.0031426 \\ 5976.6 & 1.005182 & 5977.03 & 1.0029203 \\ 5976.7 & 1.0050206 & 5977.13 & 1.0033391 \\ 5976.8 & 1.0049767 & 5977.23 & 1.0036528 \\ 5976.9 & 1.0050762 & 5977.33 & 1.0035444 \\ 5977 & 1.005482 & 5977.43 & 1.0040117 \\ 5977.1 & 1.0053207 & 5977.53 & 1.0037756 \\ 5977.2 & 1.0054135 & 5977.63 & 1.0026785 \\ 5977.3 & 1.0056177 & 5977.73 & 1.0021314 \\ 5977.4 & 1.0055937 & 5977.83 & 1.002368 \\ 5977.5 & 1.0062313 & 5977.93 & 1.0019533 \\ 5977.6 & 1.00591 & 5978.03 & 1.0007545 \\ 5977.7 & 1.0062207 & 5978.13 & 1.0008227 \\ 5977.8 & 1.0066016 & 5978.23 & 1.0005403 \\ 5977.9 & 1.0067886 & 5978.33 & 1.0000973 \\ 5978 & 1.0070074 & 5978.43 & 0.99963022 \\ 5978.1 & 1.006995 & 5978.53 & 0.99946248 \\ 5978.2 & 1.0073607 & 5978.63 & 0.99958309 \\ 5978.3 & 1.0074871 & 5978.73 & 0.99972708 \\ 5978.4 & 1.0072757 & 5978.83 & 0.99997479 \\ 5978.5 & 1.0074726 & 5978.93 & 1.0003801 \\ 5978.6 & 1.0078206 & 5979.03 & 1.0005245 \\ 5978.7 & 1.0079764 & 5979.13 & 1.0004234 \\ 5978.8 & 1.0081627 & 5979.23 & 1.0005785 \\ 5978.9 & 1.0084612 & 5979.33 & 0.99979077 \\ 5979 & 1.0089933 & 5979.43 & 0.99938051 \\ 5979.1 & 1.0091643 & 5979.53 & 0.99919211 \\ 5979.2 & 1.0094172 & 5979.63 & 0.99946587 \\ 5979.3 & 1.0095901 & 5979.73 & 0.99971135 \\ 5979.4 & 1.0096217 & 5979.83 & 0.99953427 \\ 5979.5 & 1.0093769 & 5979.93 & 1.0002829 \\ 5979.6 & 1.009748 & 5980.03 & 0.99988445 \\ 5979.7 & 1.0098058 & 5980.13 & 0.99974461 \\ 5979.8 & 1.0096622 & 5980.23 & 0.99976359 \\ 5979.9 & 1.0098139 & 5980.33 & 0.99994465 \\ 5980 & 1.0099277 & 5980.43 & 0.99983972 \\ 5980.1 & 1.0105256 & 5980.53 & 0.99988791 \\ 5980.2 & 1.0095526 & 5980.63 & 0.99988423\end{array}$




\begin{tabular}{|c|c|c|c|}
\hline 5980.3 & 1.0082577 & 5980.73 & 0.99954417 \\
\hline 5980.4 & 1.0071404 & 5980.83 & 0.99948655 \\
\hline 5980.5 & 1.0053309 & 5980.93 & 0.99982055 \\
\hline 5980.6 & 1.0038375 & 5981.03 & 1.0000124 \\
\hline 5980.7 & 1.0018654 & 5981.13 & 0.9999088 \\
\hline 5980.8 & 1.0002394 & 5981.23 & 1.0000404 \\
\hline 5980.9 & 0.99892266 & 5981.33 & 1.0003898 \\
\hline 5981 & 0.99769771 & 5981.43 & 1.0006783 \\
\hline 5981.1 & 0.99699433 & 5981.53 & 1.0009336 \\
\hline 5981.2 & 0.9958289 & 5981.63 & 1.0010725 \\
\hline 5981.3 & 0.99522206 & 5981.73 & 1.0007922 \\
\hline 5981.4 & 0.99495394 & 5981.83 & 1.0013568 \\
\hline 5981.5 & 0.99426561 & 5981.93 & 1.0015579 \\
\hline 5981.6 & 0.9942112 & 5982.03 & 1.0015908 \\
\hline 5981.7 & 0.99421631 & 5982.13 & 1.0015273 \\
\hline 5981.8 & 0.99379709 & 5982.23 & 1.0021156 \\
\hline 5981.9 & 0.99330656 & 5982.33 & 1.0021178 \\
\hline 5982 & 0.99291314 & 5982.43 & 1.0024848 \\
\hline 5982.1 & 0.99317628 & 5982.53 & 1.0022663 \\
\hline 5982.2 & 0.99242328 & 5982.63 & 1.0023603 \\
\hline 5982.3 & 0.99208839 & 5982.73 & 1.0025981 \\
\hline 5982.4 & 0.9912795 & 5982.83 & 1.0032256 \\
\hline 5982.5 & 0.99038006 & 5982.93 & 1.0040158 \\
\hline 5982.6 & 0.98796854 & 5983.03 & 1.0039269 \\
\hline 5982.7 & 0.98767485 & 5983.13 & 1.0039677 \\
\hline 5982.8 & 0.98714645 & 5983.23 & 1.0038557 \\
\hline 5982.9 & 0.98640111 & 5983.33 & 1.0037429 \\
\hline 5983 & 0.98621205 & 5983.43 & 1.0035621 \\
\hline 5983.1 & 0.98608399 & 5983.53 & 1.0036444 \\
\hline 5983.2 & 0.98555067 & 5983.63 & 1.0034603 \\
\hline 5983.3 & 0.98485845 & 5983.73 & 1.0035553 \\
\hline 5983.4 & 0.98543465 & 5983.83 & 1.0036035 \\
\hline 5983.5 & 0.98501751 & 5983.93 & 1.0030726 \\
\hline 5983.6 & 0.98558284 & 5984.03 & 1.0033622 \\
\hline 5983.7 & 0.98626375 & 5984.13 & 1.003175 \\
\hline 5983.8 & 0.98668809 & 5984.23 & 1.0030301 \\
\hline 5983.9 & 0.99102392 & 5984.33 & 1.0038012 \\
\hline 5984 & 0.9921714 & 5984.43 & 1.0040571 \\
\hline 5984.1 & 0.99281806 & 5984.53 & 1.0037964 \\
\hline 5984.2 & 0.99367344 & 5984.63 & 1.0036641 \\
\hline 5984.3 & 0.99473886 & 5984.73 & 1.0032892 \\
\hline 5984.4 & 0.99576918 & 5984.83 & 1.003016 \\
\hline 5984.5 & 0.99643322 & 5984.93 & 1.0027664 \\
\hline
\end{tabular}




\begin{tabular}{|c|c|c|c|}
\hline 5984.6 & 0.99726351 & 5985.03 & 1.0025899 \\
\hline 5984.7 & 0.99768355 & 5985.13 & 1.0025071 \\
\hline 5984.8 & 0.99827691 & 5985.23 & 1.0016119 \\
\hline 5984.9 & 0.99881275 & 5985.33 & 1.0015232 \\
\hline 5985 & 0.99901527 & 5985.43 & 1.0014777 \\
\hline 5985.1 & 0.99850115 & 5985.53 & 1.0014722 \\
\hline 5985.2 & 0.99744521 & 5985.63 & 1.0014965 \\
\hline 5985.3 & 0.9954931 & 5985.73 & 1.0011789 \\
\hline 5985.4 & 0.99119637 & 5985.83 & 1.0005187 \\
\hline 5985.5 & 0.98459889 & 5985.93 & 0.99956071 \\
\hline 5985.6 & 0.97502246 & 5986.03 & 0.99872362 \\
\hline 5985.7 & 0.96004984 & 5986.13 & 0.99757081 \\
\hline 5985.8 & 0.94492822 & 5986.23 & 0.99531048 \\
\hline 5985.9 & 0.93141255 & 5986.33 & 0.99355491 \\
\hline 5986 & 0.92188803 & 5986.43 & 0.99220355 \\
\hline 5986.1 & 0.91439737 & 5986.53 & 0.99098987 \\
\hline 5986.2 & 0.91019734 & 5986.63 & 0.99001302 \\
\hline 5986.3 & 0.9111989 & 5986.73 & 0.99111955 \\
\hline 5986.4 & 0.91789083 & 5986.83 & 0.9920781 \\
\hline 5986.5 & 0.93129293 & 5986.93 & 0.99440362 \\
\hline 5986.6 & 0.95134593 & 5987.03 & 0.9975679 \\
\hline 5986.7 & 0.98002106 & 5987.13 & 1.0022392 \\
\hline 5986.8 & 1.0071271 & 5987.23 & 1.0072882 \\
\hline 5986.9 & 1.0293007 & 5987.33 & 1.0114856 \\
\hline 5987 & 1.0435554 & 5987.43 & 1.0147869 \\
\hline 5987.1 & 1.0566296 & 5987.53 & 1.0178443 \\
\hline 5987.2 & 1.0649327 & 5987.63 & 1.0196008 \\
\hline 5987.3 & 1.0697408 & 5987.73 & 1.0199303 \\
\hline 5987.4 & 1.0718528 & 5987.83 & 1.0199713 \\
\hline 5987.5 & 1.0720233 & 5987.93 & 1.0191331 \\
\hline 5987.6 & 1.0707632 & 5988.03 & 1.0182833 \\
\hline 5987.7 & 1.0677577 & 5988.13 & 1.0170122 \\
\hline 5987.8 & 1.0627741 & 5988.23 & 1.0153238 \\
\hline 5987.9 & 1.05737 & 5988.33 & 1.0130389 \\
\hline 5988 & 1.0493336 & 5988.43 & 1.0109563 \\
\hline 5988.1 & 1.0391445 & 5988.53 & 1.008542 \\
\hline 5988.2 & 1.0283754 & 5988.63 & 1.0068388 \\
\hline 5988.3 & 1.0197388 & 5988.73 & 1.0055903 \\
\hline 5988.4 & 1.0128263 & 5988.83 & 1.0040429 \\
\hline 5988.5 & 1.0070223 & 5988.93 & 1.002924 \\
\hline 5988.6 & 1.0036836 & 5989.03 & 1.002762 \\
\hline 5988.7 & 1.0010025 & 5989.13 & 1.0021616 \\
\hline 5988.8 & 0.99911203 & 5989.23 & 1.0025295 \\
\hline
\end{tabular}




\begin{tabular}{|c|c|c|c|}
\hline 5988.9 & 0.99809999 & 5989.33 & 1.00256 \\
\hline 5989 & 0.99806238 & 5989.43 & 1.0025962 \\
\hline 5989.1 & 1.0041763 & 5989.53 & 1.0043515 \\
\hline 5989.2 & 1.0041258 & 5989.63 & 1.0043171 \\
\hline 5989.3 & 1.004055 & 5989.73 & 1.0052638 \\
\hline 5989.4 & 1.0038566 & 5989.83 & 1.0059898 \\
\hline 5989.5 & 1.0030996 & 5989.93 & 1.0061844 \\
\hline 5989.6 & 1.0034273 & 5990.03 & 1.0069234 \\
\hline 5989.7 & 1.0032357 & 5990.13 & 1.0078816 \\
\hline 5989.8 & 1.0033016 & 5990.23 & 1.0082203 \\
\hline 5989.9 & 1.0028639 & 5990.33 & 1.0084172 \\
\hline 5990 & 1.0029335 & 5990.43 & 1.0091241 \\
\hline 5990.1 & 1.0034368 & 5990.53 & 1.0092938 \\
\hline 5990.2 & 1.0032225 & 5990.63 & 1.0088347 \\
\hline 5990.3 & 1.0008914 & 5990.73 & 1.007835 \\
\hline 5990.4 & 1.0009211 & 5990.83 & 1.0076493 \\
\hline 5990.5 & 1.001238 & 5990.93 & 1.0078565 \\
\hline 5990.6 & 1.0009983 & 5991.03 & 1.0079475 \\
\hline 5990.7 & 1.0011804 & 5991.13 & 1.0082957 \\
\hline 5990.8 & 1.0011056 & 5991.23 & 1.0080629 \\
\hline 5990.9 & 1.0011521 & 5991.33 & 1.0075092 \\
\hline 5991 & 1.0009306 & 5991.43 & 1.0073714 \\
\hline 5991.1 & 1.0007456 & 5991.53 & 1.006298 \\
\hline 5991.2 & 1.0007246 & 5991.63 & 1.0065936 \\
\hline 5991.3 & 1.0008021 & 5991.73 & 1.0064374 \\
\hline 5991.4 & 1.0013376 & 5991.83 & 1.005893 \\
\hline 5991.5 & 1.0017512 & 5991.93 & 1.0056676 \\
\hline 5991.6 & 1.0024072 & 5992.03 & 1.0057578 \\
\hline 5991.7 & 1.0030069 & 5992.13 & 1.0060122 \\
\hline 5991.8 & 1.0031501 & 5992.23 & 1.0062621 \\
\hline 5991.9 & 1.0031431 & 5992.33 & 1.0060352 \\
\hline 5992 & 1.0036176 & 5992.43 & 1.0055791 \\
\hline 5992.1 & 1.0037816 & 5992.53 & 1.0055481 \\
\hline 5992.2 & 1.0041153 & 5992.63 & 1.0058852 \\
\hline 5992.3 & 1.0043105 & 5992.73 & 1.0060205 \\
\hline 5992.4 & 1.0052901 & 5992.83 & 1.0058764 \\
\hline 5992.5 & 1.0053881 & 5992.93 & 1.005655 \\
\hline 5992.6 & 1.0057913 & 5993.03 & 1.005899 \\
\hline 5992.7 & 1.0061183 & 5993.13 & 1.005708 \\
\hline 5992.8 & 1.0064842 & 5993.23 & 1.0056499 \\
\hline 5992.9 & 1.0069034 & 5993.33 & 1.005787 \\
\hline 5993 & 1.0073627 & 5993.43 & 1.0055013 \\
\hline 5993.1 & 1.0073535 & 5993.53 & 1.005276 \\
\hline
\end{tabular}




\begin{tabular}{|c|c|c|c|}
\hline 5993.2 & 1.0071154 & 5993.63 & 1.0054893 \\
\hline 5993.3 & 1.0069186 & 5993.73 & 1.0050362 \\
\hline 5993.4 & 1.0073152 & 5993.83 & 1.0056592 \\
\hline 5993.5 & 1.0068927 & 5993.93 & 1.0053691 \\
\hline 5993.6 & 1.00716 & 5994.03 & 1.0052358 \\
\hline 5993.7 & 1.007542 & 5994.13 & 1.0054633 \\
\hline 5993.8 & 1.0074124 & 5994.23 & 1.0060648 \\
\hline 5993.9 & 1.0072115 & 5994.33 & 1.0066148 \\
\hline 5994 & 1.0076007 & 5994.43 & 1.0068616 \\
\hline 5994.1 & 1.0079599 & 5994.53 & 1.0068608 \\
\hline 5994.2 & 1.0099053 & 5994.63 & 1.0071713 \\
\hline 5994.3 & 1.0104071 & 5994.73 & 1.006896 \\
\hline 5994.4 & 1.0105783 & 5994.83 & 1.0065512 \\
\hline 5994.5 & 1.010829 & 5994.93 & 1.0062173 \\
\hline 5994.6 & 1.0106551 & 5995.03 & 1.0067 \\
\hline 5994.7 & 1.0095235 & 5995.13 & 1.0068146 \\
\hline 5994.8 & 1.0084549 & 5995.23 & 1.0072963 \\
\hline 5994.9 & 1.0073516 & 5995.33 & 1.0076967 \\
\hline 5995 & 1.0054532 & 5995.43 & 1.007449 \\
\hline 5995.1 & 1.0035757 & 5995.53 & 1.0068526 \\
\hline 5995.2 & 1.0017731 & 5995.63 & 1.0067651 \\
\hline 5995.3 & 1.0006574 & 5995.73 & 1.0067924 \\
\hline 5995.4 & 0.99956361 & 5995.83 & 1.0067719 \\
\hline 5995.5 & 0.99869198 & 5995.93 & 1.006557 \\
\hline 5995.6 & 0.99816801 & 5996.03 & 1.0066342 \\
\hline 5995.7 & 0.99769589 & 5996.13 & 1.0066748 \\
\hline 5995.8 & 0.99676065 & 5996.23 & 1.0070998 \\
\hline 5995.9 & 0.99612578 & 5996.33 & 1.0069234 \\
\hline 5996 & 0.9961029 & 5996.43 & 1.0075691 \\
\hline 5996.1 & 0.99568214 & 5996.53 & 1.0073215 \\
\hline 5996.2 & 0.99612807 & 5996.63 & 1.0070216 \\
\hline 5996.3 & 0.99669124 & 5996.73 & 1.0075875 \\
\hline 5996.4 & 0.99667969 & 5996.83 & 1.0077301 \\
\hline 5996.5 & 0.99695762 & 5996.93 & 1.0074 \\
\hline 5996.6 & 0.99679243 & 5997.03 & 1.0076627 \\
\hline 5996.7 & 0.99665279 & 5997.13 & 1.0081737 \\
\hline 5996.8 & 0.99778706 & 5997.23 & 1.009525 \\
\hline 5996.9 & 0.99772755 & 5997.33 & 1.0095047 \\
\hline 5997 & 0.99799262 & 5997.43 & 1.0095868 \\
\hline 5997.1 & 0.99824383 & 5997.53 & 1.0096699 \\
\hline 5997.2 & 0.99845691 & 5997.63 & 1.0097846 \\
\hline 5997.3 & 0.99844454 & 5997.73 & 1.0098878 \\
\hline 5997.4 & 0.99846389 & 5997.83 & 1.0102295 \\
\hline
\end{tabular}




\begin{tabular}{|c|c|c|c|}
\hline 5997.5 & 0.99869878 & 5997.93 & 1.010553 \\
\hline 5997.6 & 0.99853881 & 5998.03 & 1.0101461 \\
\hline 5997.7 & 0.99865237 & 5998.13 & 1.0097627 \\
\hline 5997.8 & 0.99889859 & 5998.23 & 1.0100286 \\
\hline 5997.9 & 0.99891107 & 5998.33 & 1.0095912 \\
\hline 5998 & 0.9970233 & 5998.43 & 1.0080801 \\
\hline 5998.1 & 0.99708789 & 5998.53 & 1.0082311 \\
\hline 5998.2 & 0.99700488 & 5998.63 & 1.0080506 \\
\hline 5998.3 & 0.9972053 & 5998.73 & 1.0085824 \\
\hline 5998.4 & 0.99742197 & 5998.83 & 1.0084151 \\
\hline 5998.5 & 0.99718887 & 5998.93 & 1.0079745 \\
\hline 5998.6 & 0.99711138 & 5999.03 & 1.0075661 \\
\hline 5998.7 & 0.99731118 & 5999.13 & 1.0070201 \\
\hline 5998.8 & 0.9972482 & 5999.23 & 1.0067478 \\
\hline 5998.9 & 0.99725123 & 5999.33 & 1.0067535 \\
\hline 5999 & 0.99761483 & 5999.43 & 1.0064788 \\
\hline 5999.1 & 0.99758865 & 5999.53 & 1.0063561 \\
\hline 5999.2 & 0.99766431 & 5999.63 & 1.0064972 \\
\hline 5999.3 & 0.99781727 & 5999.73 & 1.0061338 \\
\hline 5999.4 & 1.002879 & 5999.83 & 1.0055679 \\
\hline 5999.5 & 1.0033521 & 5999.93 & 1.0049848 \\
\hline 5999.6 & 1.0033298 & 6000.03 & 1.005044 \\
\hline 5999.7 & 1.0041495 & 6000.13 & 1.0052675 \\
\hline 5999.8 & 1.0046032 & 6000.23 & 1.0057352 \\
\hline 5999.9 & 1.0039855 & 6000.33 & 1.0058603 \\
\hline 6000 & 1.0040523 & 6000.43 & 1.0050538 \\
\hline 6000.1 & 1.0041649 & 6000.53 & 1.0053153 \\
\hline 6000.2 & 1.0042246 & 6000.63 & 1.0053711 \\
\hline 6000.3 & 1.0038929 & 6000.73 & 1.0056271 \\
\hline 6000.4 & 1.0039135 & 6000.83 & 1.0056207 \\
\hline 6000.5 & 1.0042617 & 6000.93 & 1.0054232 \\
\hline 6000.6 & 1.0042361 & 6001.03 & 1.0054163 \\
\hline 6000.7 & 1.0045251 & 6001.13 & 1.0057366 \\
\hline 6000.8 & 1.004708 & 6001.23 & 1.0054691 \\
\hline 6000.9 & 1.0049941 & 6001.33 & 1.0052522 \\
\hline 6001 & 1.0048389 & 6001.43 & 1.0050383 \\
\hline 6001.1 & 1.0048655 & 6001.53 & 1.0049725 \\
\hline 6001.2 & 1.0049811 & 6001.63 & 1.0050979 \\
\hline 6001.3 & 1.0048719 & 6001.73 & 1.0051915 \\
\hline 6001.4 & 1.0046938 & 6001.83 & 1.0048676 \\
\hline 6001.5 & 1.0040745 & 6001.93 & 1.0044837 \\
\hline 6001.6 & 1.0031845 & 6002.03 & 1.0050175 \\
\hline 6001.7 & 1.0023734 & 6002.13 & 1.0047074 \\
\hline
\end{tabular}




\begin{tabular}{|c|c|c|c|}
\hline 6001.8 & 1.0005305 & 6002.23 & 1.0043969 \\
\hline 6001.9 & 0.99877087 & 6002.33 & 1.0046171 \\
\hline 6002 & 0.9999804 & 6002.43 & 1.0047807 \\
\hline 6002.1 & 0.99826949 & 6002.53 & 1.0048327 \\
\hline 6002.2 & 0.99701757 & 6002.63 & 1.0051022 \\
\hline 6002.3 & 0.99630153 & 6002.73 & 1.0056094 \\
\hline 6002.4 & 0.99543656 & 6002.83 & 1.0056729 \\
\hline 6002.5 & 0.99450043 & 6002.93 & 1.006437 \\
\hline 6002.6 & 0.99405312 & 6003.03 & 1.005995 \\
\hline 6002.7 & 0.9930481 & 6003.13 & 1.0066618 \\
\hline 6002.8 & 0.99262306 & 6003.23 & 1.0067879 \\
\hline 6002.9 & 0.99199415 & 6003.33 & 1.0065496 \\
\hline 6003 & 0.991539 & 6003.43 & 1.0062451 \\
\hline 6003.1 & 0.99158225 & 6003.53 & 1.0062602 \\
\hline 6003.2 & 0.99139478 & 6003.63 & 1.0070585 \\
\hline 6003.3 & 0.99172192 & 6003.73 & 1.0066526 \\
\hline 6003.4 & 0.99141118 & 6003.83 & 1.0064359 \\
\hline 6003.5 & 0.99115718 & 6003.93 & 1.0062439 \\
\hline 6003.6 & 0.99107048 & 6004.03 & 1.0063899 \\
\hline 6003.7 & 0.99053727 & 6004.13 & 1.0061065 \\
\hline 6003.8 & 0.99000913 & 6004.23 & 1.0060271 \\
\hline 6003.9 & 0.98965758 & 6004.33 & 1.0058877 \\
\hline 6004 & 0.98957306 & 6004.43 & 1.006378 \\
\hline 6004.1 & 0.9895806 & 6004.53 & 1.006575 \\
\hline 6004.2 & 0.98931492 & 6004.63 & 1.0063379 \\
\hline 6004.3 & 0.98925595 & 6004.73 & 1.0064346 \\
\hline 6004.4 & 0.98899207 & 6004.83 & 1.006199 \\
\hline 6004.5 & 0.98931583 & 6004.93 & 1.0064489 \\
\hline 6004.6 & 0.99074586 & 6005.03 & 1.0066945 \\
\hline 6004.7 & 0.99042221 & 6005.13 & 1.0070967 \\
\hline 6004.8 & 0.99045286 & 6005.23 & 1.0071349 \\
\hline 6004.9 & 0.99047039 & 6005.33 & 1.0071373 \\
\hline 6005 & 0.98991743 & 6005.43 & 1.0066873 \\
\hline 6005.1 & 0.98943975 & 6005.53 & 1.0064324 \\
\hline 6005.2 & 0.9894847 & 6005.63 & 1.0058897 \\
\hline 6005.3 & 0.99018612 & 6005.73 & 1.0061296 \\
\hline 6005.4 & 0.99050844 & 6005.83 & 1.0066411 \\
\hline 6005.5 & 0.9909976 & 6005.93 & 1.0072093 \\
\hline 6005.6 & 0.99110283 & 6006.03 & 1.0073857 \\
\hline 6005.7 & 0.99199092 & 6006.13 & 1.0071225 \\
\hline 6005.8 & 0.9912485 & 6006.23 & 1.0070973 \\
\hline 6005.9 & 0.99198661 & 6006.33 & 1.006911 \\
\hline 6006 & 0.99253256 & 6006.43 & 1.0075673 \\
\hline
\end{tabular}




$\begin{array}{cccc}6006.1 & 0.99287857 & 6006.53 & 1.0078462 \\ 6006.2 & 0.99399872 & 6006.63 & 1.0077321 \\ 6006.3 & 0.99503881 & 6006.73 & 1.0077015 \\ 6006.4 & 0.99569393 & 6006.83 & 1.0078796 \\ 6006.5 & 0.99613604 & 6006.93 & 1.0080148 \\ 6006.6 & 0.99681364 & 6007.03 & 1.0084251 \\ 6006.7 & 0.9976684 & 6007.13 & 1.0085158 \\ 6006.8 & 0.99755628 & 6007.23 & 1.0085472 \\ 6006.9 & 0.99780267 & 6007.33 & 1.008826 \\ 6007 & 0.99838067 & 6007.43 & 1.008677 \\ 6007.1 & 0.99853694 & 6007.53 & 1.0088618 \\ 6007.2 & 0.9990229 & 6007.63 & 1.0107861 \\ 6007.3 & 0.99970223 & 6007.73 & 1.0110513 \\ 6007.4 & 0.99960791 & 6007.83 & 1.010653 \\ 6007.5 & 0.99970824 & 6007.93 & 1.0107168 \\ 6007.6 & 0.9999407 & 6008.03 & 1.0109436 \\ 6007.7 & 0.99996637 & 6008.13 & 1.0106321 \\ 6007.8 & 0.9994423 & 6008.23 & 1.0104535 \\ 6007.9 & 0.99904577 & 6008.33 & 1.010343 \\ 6008 & 0.99912213 & 6008.43 & 1.0100306 \\ 6008.1 & 0.99882298 & 6008.53 & 1.0100265 \\ 6008.2 & 0.99887157 & 6008.63 & 1.0090574 \\ 6008.3 & 0.99852437 & 6008.73 & 1.0092131 \\ 6008.4 & 0.9988423 & 6008.83 & 1.0090604 \\ 6008.5 & 0.99961416 & 6008.93 & 1.0091554 \\ 6008.6 & 0.99927956 & 6009.03 & 1.0088762 \\ 6008.7 & 0.99905563 & 6009.13 & 1.0085925 \\ 6008.8 & 0.99950754 & 6009.23 & 1.0082904 \\ 6008.9 & 0.99917852 & 6009.33 & 1.007973 \\ 6009 & 0.99880519 & 6009.43 & 1.007851 \\ 6009.1 & 0.99819551 & 6009.53 & 1.0082746 \\ 6009.2 & 0.99738024 & 6009.63 & 1.0078711 \\ 6009.3 & 0.99621077 & 6009.73 & 1.0076575 \\ 6009.4 & 0.99496448 & 6009.83 & 1.0077092 \\ 6009.5 & 0.99376688 & 6009.93 & 1.0079796 \\ 6009.6 & 0.99243711 & 6010.03 & 1.0084157 \\ 6009.7 & 0.99125046 & 6010.13 & 1.0086214 \\ 6009.8 & 0.99631699 & 6010.23 & 1.0089271 \\ 6009.9 & 0.99619057 & 6010.33 & 1.008899 \\ 6010 & 0.99532264 & 6010.43 & 1.0089044 \\ 6010.1 & 0.99459881 & 6010.53 & \end{array}$

